\title{
Engineered Inks for Environment-Friendly Additive Manufacturing of Hierarchical Microstructures
}

\author{
Maria Alejandra Torres Arango
}

Follow this and additional works at: https://researchrepository.wvu.edu/etd

\section{Recommended Citation}

Torres Arango, Maria Alejandra, "Engineered Inks for Environment-Friendly Additive Manufacturing of Hierarchical Microstructures" (2017). Graduate Theses, Dissertations, and Problem Reports. 7139. https://researchrepository.wvu.edu/etd/7139

This Dissertation is protected by copyright and/or related rights. It has been brought to you by the The Research Repository @ WVU with permission from the rights-holder(s). You are free to use this Dissertation in any way that is permitted by the copyright and related rights legislation that applies to your use. For other uses you must obtain permission from the rights-holder(s) directly, unless additional rights are indicated by a Creative Commons license in the record and/ or on the work itself. This Dissertation has been accepted for inclusion in WVU Graduate Theses, Dissertations, and Problem Reports collection by an authorized administrator of The Research Repository @ WVU. For more information, please contact researchrepository@mail.wvu.edu. 


\title{
Engineered Inks for Environment-Friendly Additive Manufacturing of Hierarchical Microstructures
}

\author{
Maria Alejandra Torres Arango
}

Thesis submitted

to the Statler College of Engineering and Mineral Resources at West Virginia University

in partial fulfilment of the requirements for the degree of

Doctor of Philosophy in

Materials Science \& Engineering

Konstantinos A. Sierros, Ph.D., Chair

Edward Sabolsky, Ph.D.

Terence Musho, Ph.D.

Dimitris Korakakis, Ph.D.

Charter Stinespring, Ph.D.

Department of Mechanical \& Aerospace Engineering

\section{Morgantown, West Virginia}

2017

Keywords: Direct Ink Writing, Ink Synthesis, Microstructure Engineering, Sustainable Ink Processing, Hierarchical Cellular Architectures, Secondary-Phase Decorated Composites.

Copyright 2017 Maria Alejandra Torres Arango 


\section{ABSTRACT \\ Engineered Inks for Environment-Friendly Additive Manufacturing of Hierarchical Microstructures}

\section{Maria Alejandra Torres Arango}

Novel material synthesis and green engineering innovations in manufacturing are important enablers of new technologies. Here, a range of functional materials systems and their processing through additive manufacturing are investigated. The materials studied consist of $\mathrm{Ag}, \mathrm{ZnO}$ and $\mathrm{TiO}_{2}$ based inks for additive manufacturing with potential application across multiple technological fields.

In particular, the synthesis of multi-functional emulsion inks, and their deposition through continuous-flow direct writing are investigated. Highlights of this work include the study of the curing/sintering conditions of such ink systems aiming for fundamental insights in their synthesis at low-temperatures (below $150^{\circ} \mathrm{C}$ ), compatible with flexible polymeric substrates. Also, the investigation of texturing and microstructural control via ink composition, direct writing conditions and curing/sintering treatments is reported. In addition, the design and investigation of hybrid (organic/inorganic) multiphase composite ink systems for the realization of planar and 3D printable cellular structures, is discussed. Furthermore, the nucleation or decoration of the latter, via encapsulation of the decorative material and/or their precursors in one of the phases of the synthesized ink is investigated.

Special emphasis is placed on an environmentally-friendly approach for the realization of sustainable inks, aiming for industry-transferable developments. Such approach provides the potential to further additive manufacturing and its utilization in a plethora of technological applications; ranging from energy, to waste management and water cleaning, to biomedical. 
To my Family 


\section{List of Publications}

1. "Direct Writing and Electro-Mechanical Characterization of Ag Micro-Patterns on Polymer Substrates for Flexible Electronics" Torres Arango, M. A.; Cokeley, A. M.; Beard, J. J.; Sierros, K. A. Thin Solid Films 2015, 596, 167-173

2. "Robotic Deposition of $\mathrm{TiO}_{2}$ Films on Flexible Substrates from Hybrid Inks: Investigation of Synthesis-Processing-Microstructure-Photocatalytic Relationships" Torres Arango, M. A.; Valença de Andrade, A. S.; Cipollone, D. T.; Grant, L. O.; Korakakis, D.; Sierros, K. A. ACS Appl. Mater. Interfaces 2016, 8 (37), 24659-24670.

3. "Continuous-Flow Direct Writing of Hybrid $\mathrm{TiO}_{2}$ Flexible Photo-Electrodes: Processing, Microstructure and Functionality Interrelations" Torres Arango, M. A.; Cipollone, D. T.; Grant, L. O.; Korakakis, D.; Sierros, K. A. MRS Adv. 2017, 2 (18), 1021-1028

4. "Tuning the Crystalline Microstructure of Al-Doped ZnO Using Direct Ink Writing" Torres Arango, M. A.; Abidakun, O. A.; Korakakis, D.; Sierros, K. A. Flex. Print. Electron. 2017; DOI: 10.1088/2058$8585 /$ aa8801

5. "Environment-Friendly Engineering \& 3D Printing of $\mathrm{TiO}_{2}$ Hierarchical Mesoporous Cellular Architectures" ACS Sustainable Chem. Eng. 2017; DOI: 10.1021/acssuschemeng.7b02450

6. Ag-decorated $\mathrm{TiO}_{2}$ Foams and Structures - In preparation, Materials Horizons 


\section{Table of Contents}

ABSTRACT

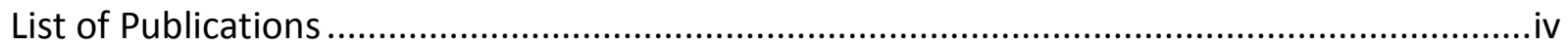

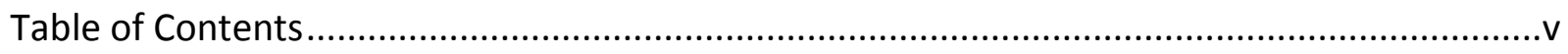

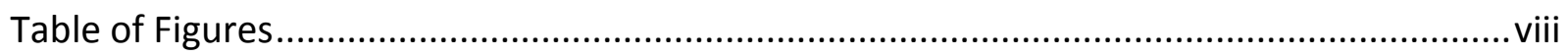

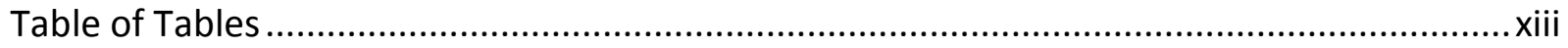

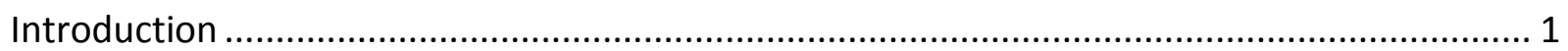

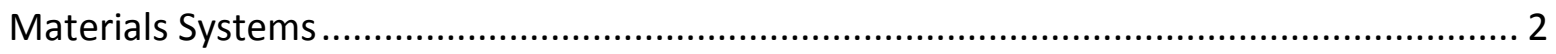

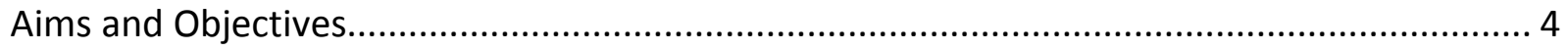

Aims

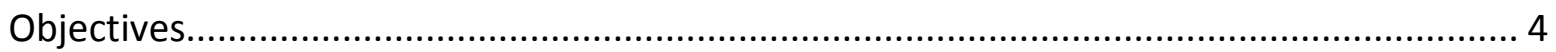

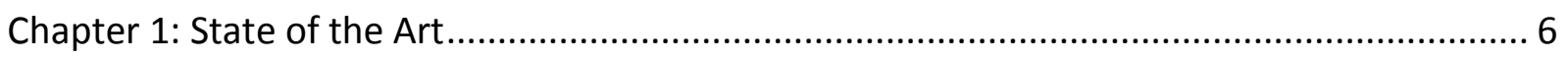

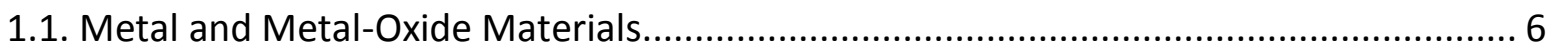

1.2. Additive Manufacturing using Metal and Metal-Oxide Inks.......................................... 7

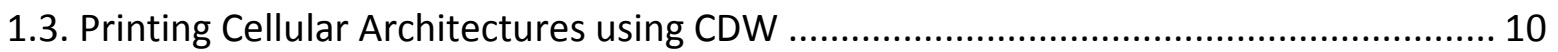

1.4. Sustainability Based Additive Manufacturing ........................................................... 11

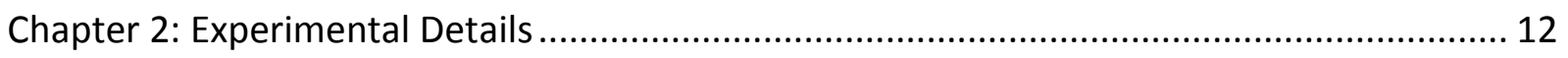

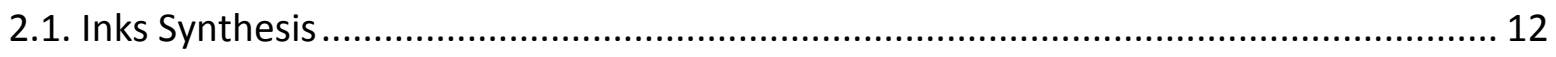

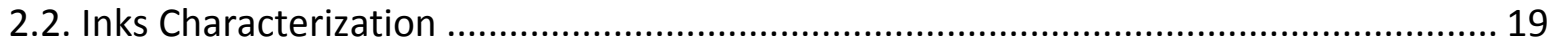

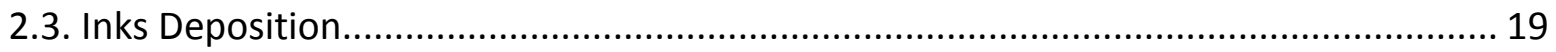

2.4. Films/Patterns Characterization ......................................................................... 20

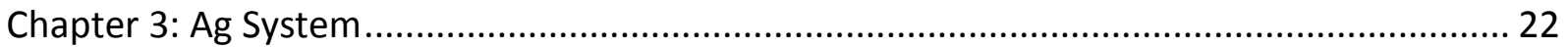

3.1. Ag Particle Nucleation Process: Influence of pH, Viscosity and Temperature .............. 22

3.2. Ink Properties and Robotic Deposition ........................................................................ 24

3.3. Patterns Microstructure and Electromechanical Properties ......................................... 25

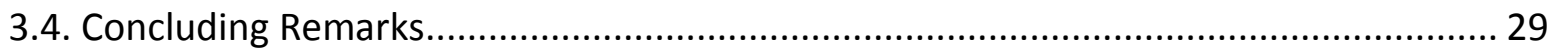

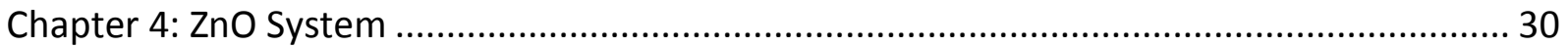

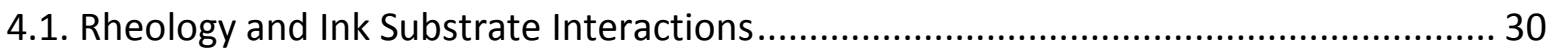

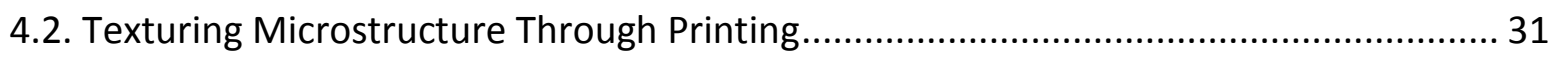

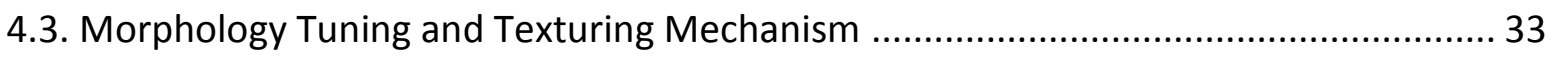

4.4. Illustration of Films Mechanical Flexibility ............................................................... 37

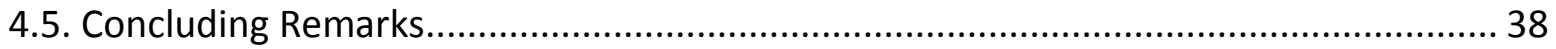




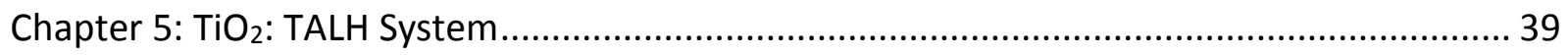

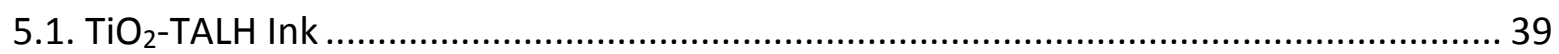

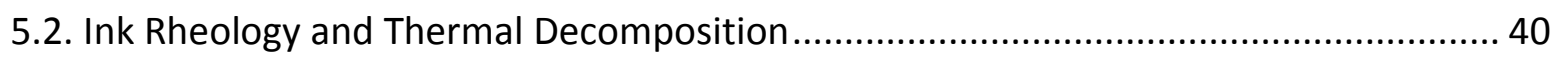

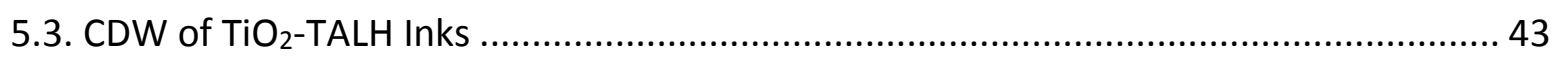

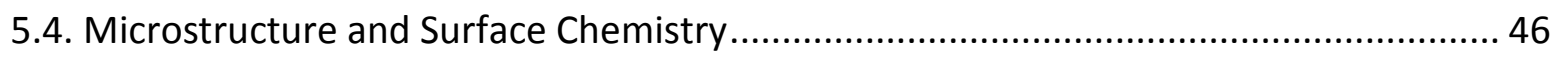

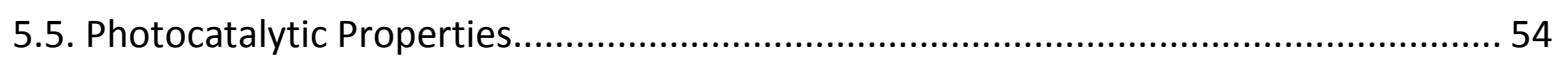

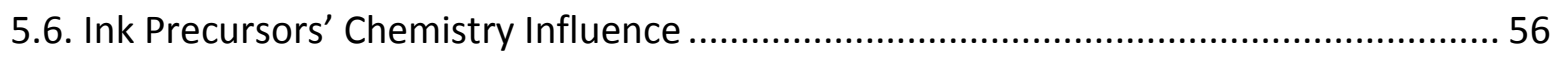

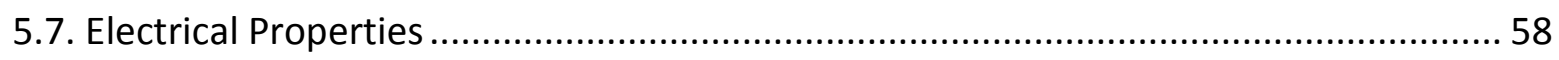

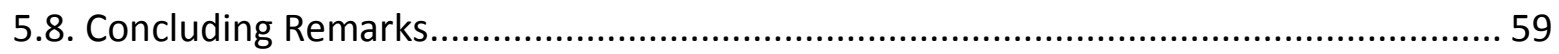

Chapter 6: Multiphase Materials Systems I: Microstructuring and Enabling 3D Printing of $\mathrm{TiO}_{2}$

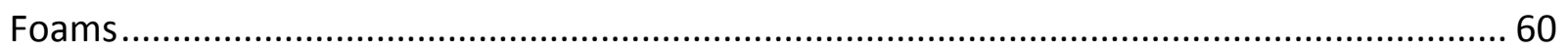

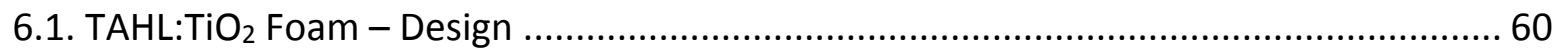

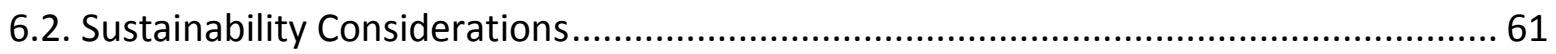

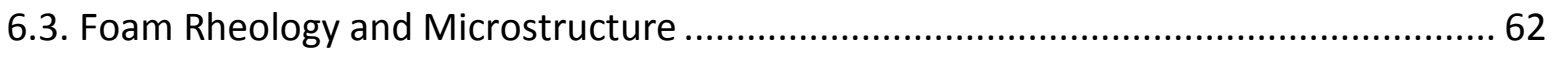

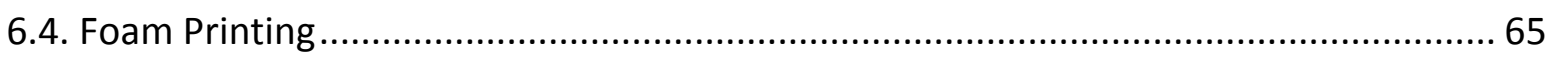

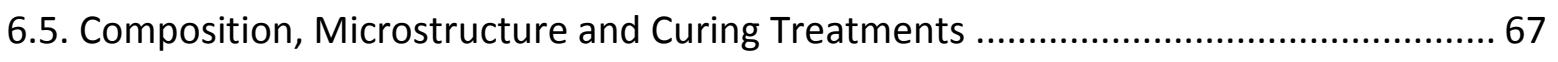

6.6. Microstructural, Compositional and Photocatalytic Relationships ............................... 73

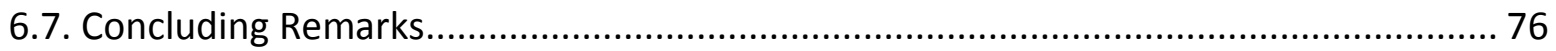

Chapter 7: Multiphase Materials Systems II: Encapsulating Materials and Controlling the

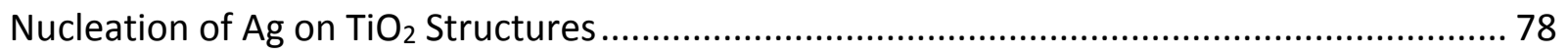

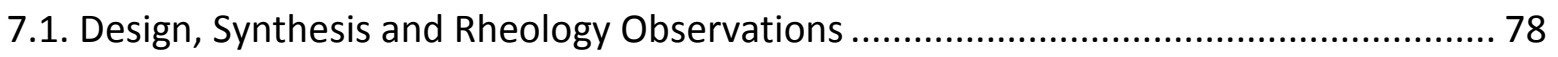

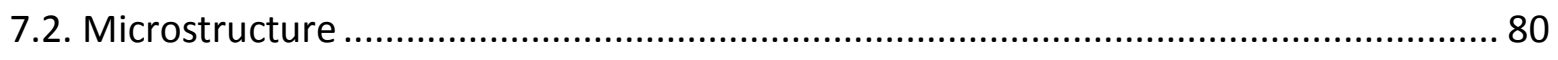

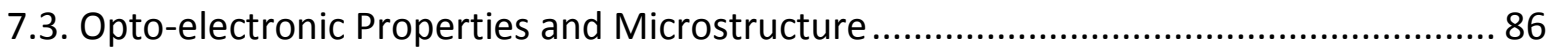

7.4. Chemical State of Nucleated Secondary-Phase Nanoparticles ...................................... 92

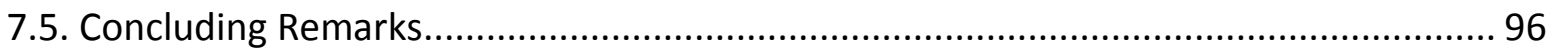

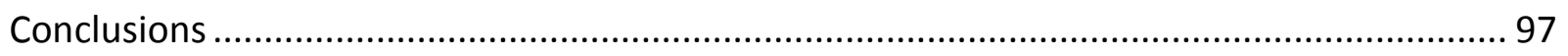

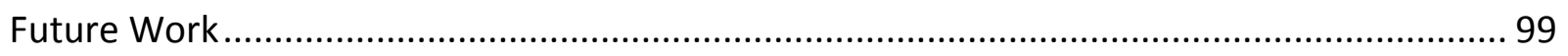

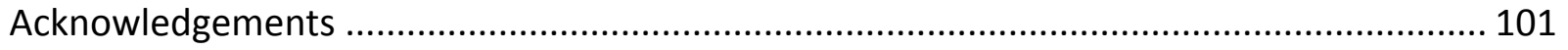

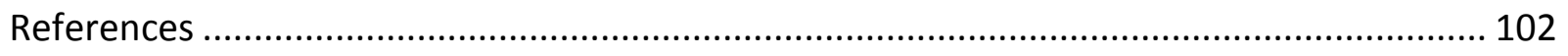

Appendix A. Additional Characterization Data for the Ag-decorated $\mathrm{TiO}_{2}$ Composites: EDS, XPS.

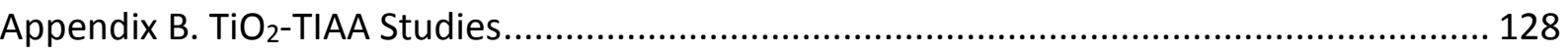

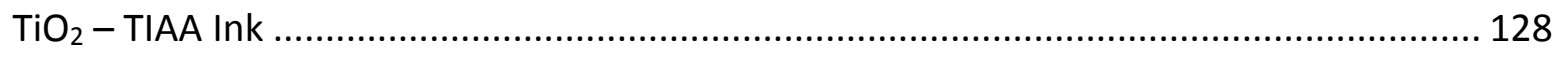




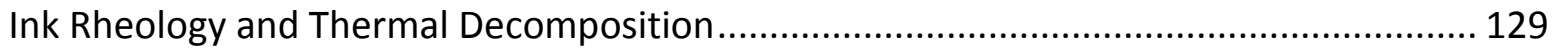

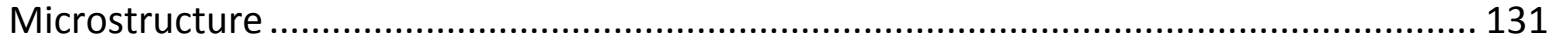

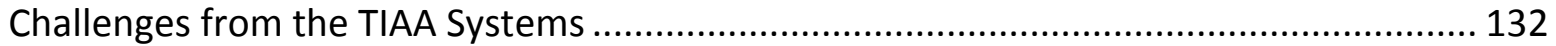

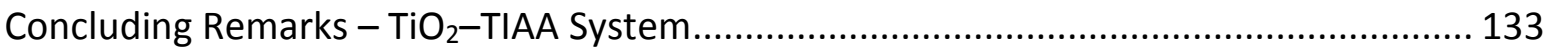




\section{Table of Figures}

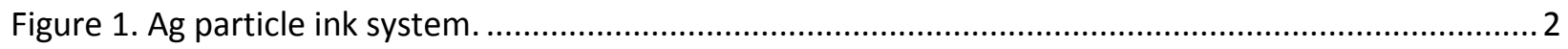

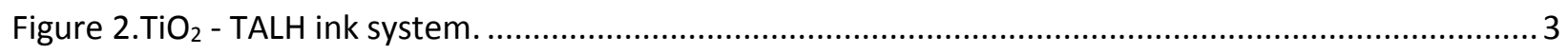

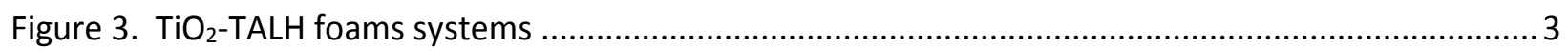

Figure 4. Continuous-flow direct writing technique schematic. Distance to substrate is enlarged for

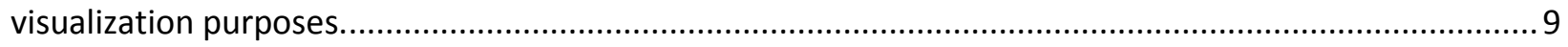

Figure 5. Ag- Ink synthesis process and precursor chemicals....................................................... 12

Figure 6. Schematic of the synthesis process for the TALH:TiO $\mathrm{Tin}_{2}$ ins and picture of a vial containing one of

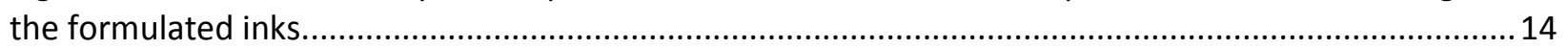

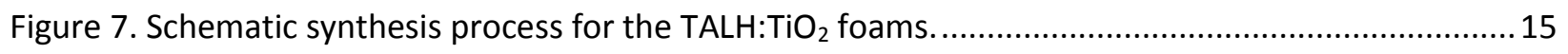

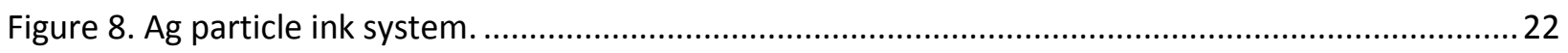

Figure 9. SEM image of the as prepared silver nanoparticles from DEA solutions. .................................23

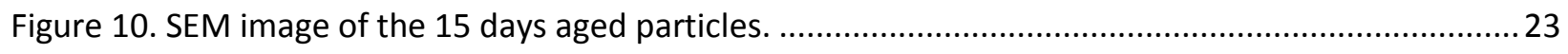

Figure 11. Viscosity as a function of shear rate for the prepared Ag ink............................................24

Figure 12. Ag-printed patterns on PEN. Effect of printing speed and pressure. ..................................24

Figure 13. Transmitted light (UV-Vis measurements) and corresponding patterns with line width of 300

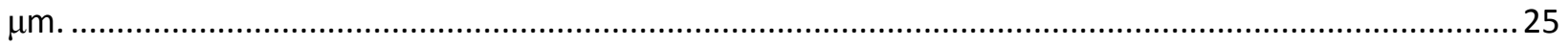

Figure 14. Electrical resistivity of the Ag patterns as measured by Hall Effect.......................................26

Figure 15. SEM images of the patterns surface after different heat treatments..................................26

Figure 16. (a) XRD patterns of the CDW Ag arrays after different annealing treatments and (b) Gaussian

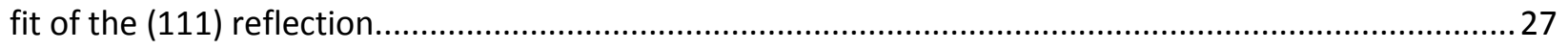

Figure 17. Optical images of the printed line pattern under monotonic tensile stress..........................27

Figure 18. Change in electrical resistance of the printed line pattern as a function of cyclic tensile stress loading.

Figure 19. Optical microscope and SEM images of the printed line pattern (a) before and (b) after cyclic tensile stress characterization.

Figure 20. (a) Dynamic viscosity versus shear rate, and (b) shear-stress variation with shear-rate for the AZO inks with different PVP content. 
Figure 21.Dependence of printed feature's width (a), and spreading (b) on writing speed and volumetric flow rate.

Figure 22. XRD patterns of films printed from ( $a-b) 15 w t \%$ PVP inks at (a) $150 \mu \mathrm{m}$ nozzle, $3 \mathrm{~mm} / \mathrm{s}$ speed, and variable pressure; and (b) $200 \mu \mathrm{m}$ nozzle, $103 \mathrm{kPa}$ pressure, and variable speed; and of films printed at various speeds with $150 \mu \mathrm{m}$ nozzles and $103 \mathrm{kPa}$ from (c) $10 \mathrm{wt} \%$ PVP and (d) $20 \mathrm{wt} \%$ PVP inks........ 32

Figure 23. Lotgering factor for (0002) reflections dependence on the width of the directly written AZO

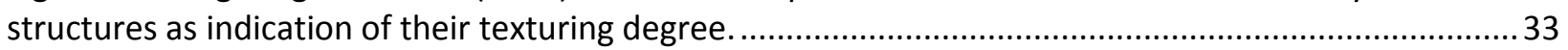

Figure 24.TGA profiles of Al-doped ZnO inks with 10, 15, and 20 wt\% PVP content. .............................34

Figure 25. XRD patterns and SEM images of the AZO $15 \mathrm{wt} \%$ structures printed on glass at $3 \mathrm{~mm} / \mathrm{s}$ and 103 $\mathrm{kPa}$, and annealed for $20 \mathrm{~min}$ at different temperatures. Arrows indicate wrinkle features; scale bars correspond to $1 \mu \mathrm{m}$. .34

Figure 26 Morphological and crystalline evolution schematic of the directly written Al-doped ZnO films. 36

Figure 27. Mechanical testing and optical microscopy images of a typical AZO structure from 15 wt\% PVP ink. (a) Side-view of film during bending; scale bar is $10 \mathrm{~mm}$. (b) Top-view of the film surface showing the appearance of a crack perpendicular to the printing direction (and applied bending stress) at $69.6 \mathrm{~mm}$ radius; scale bar corresponds to $200 \mu \mathrm{m}$. (c-i) Higher resolution images of the region showing the crack. Images (d) and (h) exhibit the lower end; and images (e) and (i) the upper end of the crack. Scale bars (ce) and (f-i), are $100 \mu \mathrm{m}$ and $20 \mu \mathrm{m}$ respectively. Arrows in (f) indicate delamination sites. 37

Figure 28. $\mathrm{TiO}_{2}$ - TALH ink system. 40

Figure 29. Viscosity of $\mathrm{TALH}: \mathrm{TiO}_{2}$ systems (a) without polymers and (b) with polymers; open symbols correspond to (1:6), and filled symbols to (1:12) formulations respectively. 41

Figure 30. Photograph of a doctor bladed $\mathrm{TiO}_{2}$ film from a low viscosity ink formulation (TALH:TiO 2 1:4.5 no-polymer) exhibiting strong particle aggregation.

Figure 31. TGA of TALH:TiO 2 systems (a) without polymers and for (b) 1:6 different polymer formulations.

Figure 32. Direct writing parameter maps for the different $\mathrm{TALH}: \mathrm{TiO}_{2}$ polymer added inks on ITO/PET substrates.

Figure 33. Contact angle of (a) different particle concentration inks on glass and PEN substrates; and of (b) polymer added formulations on ITO/PET substrates. 45

Figure 34. SEM images of the surface of (a) directly written $\mathrm{TiO}_{2}$ films, from $\mathrm{TALH}: \mathrm{TiO}_{2}$ (1:12) precursor inks; and (b) of doctor bladed (DB) films, from (1:6) and (1:12) TALH: $\mathrm{TiO}_{2}$ formulations on $\mathrm{Ag} / \mathrm{PEN}$ after 6h UV exposure. 46

Figure 35. XRD of (a) $\mathrm{TiO}_{2}$ Aeroxide ${ }^{\circledR}$ and the different particle concentration films and (b) of the $\mathrm{TALH}: \mathrm{TiO}_{2}$ (1:6) films with various polymers, after different UV treatments: No Treatment (thin-bottom 
line), $2 \mathrm{~h}$ UV (medium) and 4hUV (dark-top line). (c) Crystallite size change upon different energy input conditions, variation calculated with respect to non-treated samples.

Figure 36. XRD spectrum of the $500^{\circ} \mathrm{C}$ calcined TALH powder sample.

Figure 37. Visible Raman $\lambda=532 \mathrm{~nm}$ for the as dried (No treatment) TALH:TiO ${ }_{2}$ inks (a) inorganic region (b) organic region, as dried (No treatment) different polymer formulated inks (d) inorganic region and (e) organic region. Integrated Raman Intensity ratios for (c) different particle loading inks and (f) different polymer formulated inks as dried and after $2 \mathrm{~h}$ UV treatment. Open Symbols (No Treatment), filled symbols (2hUV). Inset in (c) depicts the integration areas: Anatase $\left(395 \mathrm{~cm}^{-1}\right)$ and Rutile $\left(445 \mathrm{~cm}^{-1}\right)$ peaks. 48

Figure 38. Optical and SEM images of the directly written TALH:TiO ${ }_{2}$ films and patterns from different polymer formulated (a) 1:6 PAA and (b) (1:12) inks on Ag-PEN substrates. With CDW speed of $5 \mathrm{~mm} / \mathrm{s}$ and pressure of $20 \mathrm{KPa}$ and $150 \mu \mathrm{m}$ inner diameter nozzles. 51

Figure 39. TEM images of the (a) as received $\mathrm{TiO}_{2}$ Aeroxide particles, (b) TALH:TiO 2 (1:6) - PAA film after 4h UV exposure, and (c) TALH:TiO ${ }_{2}$ (1:6) film after $150^{\circ} \mathrm{C}-30$ min treatment. HR-TEM images of the enclosed regions show the lattice fringes of the crystals and the bridging formations from TAHL in (b) and (c). Diffraction pattern taken for the enclosed region in (c). 52

Figure 40. X-ray photoelectron spectroscopy of the films exposed to different UV conditions (a) O1s detailed scan for (1:12) no-polymer films; (b) C1s and (c) Ti2p detailed scans. The cyan line in (c) corresponds to the experimental XPS data. 53

Figure 41. Detailed scan of Ti2p peak for the 1:12 TALH:TiO ${ }_{2}$ films from No polymer, PAA and PVP formulations. The cyan line indicates corresponds to the raw XPS data. .54

Figure 42. (a) Methylene blue (MB) degradation (\%) and (b) linearized MB concentration change in time,

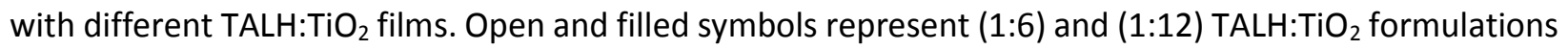
respectively. Star symbols represent $6 \mathrm{~h}$ UV treatment and square symbols represent $150{ }^{\circ} \mathrm{C}-30 \mathrm{~min}$ annealing. (c) Image of the MB initial and aliquots of the decomposed MB solutions after $150 \mathrm{~min}$ UV irradiation. .56

Figure 43. Chemical structure of the Titanium(IV) bis(ammonium lactato) dihydroxide (TALH) compound.

Figure 44. IV Characteristics of the no-polymer and PAA TALH:TiO ${ }_{2}$ films treated under $150^{\circ} \mathrm{C}-30 \mathrm{~min}$ and $6 \mathrm{~h}$ UV light $(\lambda=254 \mathrm{~nm})$; and (b) schematic of the samples and the probing sites used for IV characterization. .58

Figure 45. Schematic synthesis route, and microstructure (photograph), of mesoporous $\mathrm{TiO}_{2}$ foams.....61

Figure 46. Optical images of the 1:12 $\mathrm{TALH} \mathrm{TiO}_{2}$ L75-S5.5-019.5 MEA foam inside a polystyrene cuvette for stability assessment. (a) top and (c) bottom sections as placed in the cuvette, and (b) top and (d) bottom sections after 4 days. The cuvette width is of $10 \mathrm{~mm}$. 62

Figure 47. Optical microscope images of the foams surface and inner sections obtained from formulations (a) L75-S3-O22 and (b) L86-S3-O11, and treated at $150{ }^{\circ} \mathrm{C}-30 \mathrm{~min}$ 63 
Figure 48. Viscosity of the 1:12 $\mathrm{TALH}_{\mathrm{TiO}}$ MEA foams (a) with varying liquid-solid-oil L-S-O amounts in vol\%; and (b) L75-S3-022 foam as prepared and after 1 day of preparation with and without re-frothing. Frothing time of $4 \mathrm{~min}$. 64

Figure 49. (a) Viscosity and (b) shear-stress as a function of shear-rate for the TALH:TiO $1: 12$ foams after $8 \mathrm{~min}$ frothing time from different liquid-solid-oil content (vol\%) formulations. 64

Figure 50. (a) Printing space for the investigated $\mathrm{TiO}_{2}$ foam systems using $580 \mu \mathrm{m}$ nozzles inner diameter at $500 \mu \mathrm{m}$ dispensing height. Printing 3-dimensional layered hollow columns (b) and (d), and spanning foam structures (c), at $9.6 \mathrm{kPa}$ and $5 \mathrm{~mm} / \mathrm{s}$ on hydrophobically treated glass. Scale bars are $1 \mathrm{~mm}$ long. 66

Figure 51. Low-magnification optical microscope and SEM images of the open-cell (a-c) and (j-I); and closed-cell foams (d-i). Printing direction is from right to left. Scale bars correspond to $200 \mu \mathrm{m}$ for optical microscope images, and to $100 \mu \mathrm{m}$ for SEM images (c, f, i, and I). 68

Figure 52. Scanning electron microscope images of the macro-pores inner surfaces for the different foams systems studied and sintered at $500^{\circ} \mathrm{C}$.

Figure 53. Thermo-gravimetric analysis profiles for the different foam formulations. .70

Figure 54. Microstructure of the surface and inner regions of the L86-S3-011 MEA foam, treated at (a) $150^{\circ} \mathrm{C}-30 \mathrm{~min}$ and (b) $6 \mathrm{~h}$ UV $(\lambda=254 \mathrm{~nm})$.

Figure 55. (a) Linearized methylene blue concentration change in time, undergoing heterogeneous photocatalytic degradation in the presence of the different $\mathrm{TiO}_{2}$ foams under UV light exposure at $\lambda=254 \mathrm{~nm}$. (b) Photograph of cuvettes with degraded methylene blue solutions after $200 \mathrm{~min}$ of UV exposure. 73

Figure 56. (a) Barrett-Joyner-Halenda (BJH) cumulative micro-pore area (black) and micro-pore area distribution (blue); and (b) Horvath-Kawazoe micro-pore volume (black) and micro-pore volume distribution (green), for the primary $\mathrm{TiO}_{2}$ nanoparticles and studied foam systems. The amount of liquidsolid-oil (L-S-O) is in vol\%. 74

Figure 57. X-ray diffraction patterns of the studied foams and primary $\mathrm{TiO}_{2}$ nanoparticles; (b) main diffraction peaks corresponding to anatase and rutile crystalline structures. The amount of liquid-solid-oil (L-S-O) is in vol\%. (1:6) refers to $\mathrm{TALH}: \mathrm{TiO}_{2}$ mole ratio. 75

Figure 58. Viscosity of (a) representative TEA (no-Ag $\bullet$ ) and Ag-decorated $\mathrm{TiO}_{2}(\star)$ ) oil-based foam-ink; and (b) Ag-decorated $\mathrm{TiO}_{2}(\star)$ oil-based foam-ink, Xanthan Gum baseline ( $\left.\mathbf{\square}\right)$, and Xanthan Gum-based Agdecorated $\mathrm{TiO}_{2}(\square)$ precursor inks. 79

Figure 59. Optical microscope images of the Ag-decorated $\mathrm{TiO}_{2}$ samples from (a) oil-based, and xanthan gum-based formulations (b) and (c). Scale bars are $200 \mu \mathrm{m}$ long. Samples (a) and (b) were doctor bladed; sample (c) as scooped and allowed to relax as it dried. 80

Figure 60. SEM images of the $\mathrm{L75}-\mathrm{S} 3-\mathrm{O} 22 \mathrm{Ag}$-decorated $\mathrm{TiO}_{2}$ foam composites treated at different temperature and UV light $(\lambda=254 \mathrm{~nm})$ conditions for $20 \mathrm{~min}$. 
Figure 61. SEM images of the Xanthan gum-based $\mathrm{Ag}$-decorated $\mathrm{TiO}_{2}$ composites treated at different temperature and UV light $(\lambda=254 \mathrm{~nm})$ conditions for $20 \mathrm{~min}$.

Figure 62. SEM images of the Xanthan gum-based $\mathrm{TiO}_{2}$ baseline composites, treated at $150^{\circ} \mathrm{C}$ and UV-light $(\lambda=254 \mathrm{~nm})$ for $20 \mathrm{~min}$.

Figure 63. XRD patterns of the $\mathrm{L} 75-\mathrm{S} 3-\mathrm{O} 22 \mathrm{Ag}-\mathrm{TiO}_{2}$ foam composites treated at different temperature and UV light conditions. (b) Detailed XRD regions where the metallic Ag peak would show.

Figure 64. XRD patterns of the xanthan gum based $\mathrm{TiO}_{2}$ inks ( $a$ and $c$ ) Ag-decorated and ( $b$ and $d$ ) without $\mathrm{Ag}$ (baseline xanthan gum- $\mathrm{TiO}_{2}$ ). (c and d) Detailed XRD regions where the metallic Ag peak would show.

Figure 65. EDS point analysis and mapping of the oil-based Ag-decorated $\mathrm{TiO}_{2}$ foam treated at $300^{\circ} \mathrm{C}$ for $20 \mathrm{~min}$.

Figure 66. EDS point analysis and mapping of the xanthan gum-based $\mathrm{Ag}$-decorated $\mathrm{TiO}_{2}$ composite treated with UV-light $(\lambda=254 \mathrm{~nm})$ for $20 \mathrm{~min}$.

Figure 67. Photograph of the doctor bladed $\mathrm{Ag}$-decorated $\mathrm{TiO}_{2}$ samples treated under different energy conditions.

Figure 68.(a) Tauc plots for the determination of the optical bandgap, (b) normalized UV-Vis spectra of the differently treated oil-based $\mathrm{Ag}-\mathrm{TiO}_{2}$ foams. 88

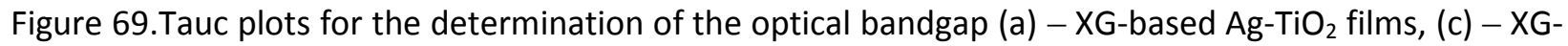
based $\mathrm{TiO}_{2}$ films; and normalized UV-Vis spectra (b) - XG-based $\mathrm{Ag}-\mathrm{TiO}_{2}$ films, (d) - XG-based $\mathrm{TiO}_{2}$ films, treated under different energy conditions. 88

Figure 70. TEM images of the oil-based $500^{\circ} \mathrm{C}$ treated $\mathrm{Ag}-\mathrm{TiO}_{2}$ foams (a) and (b); and xanthan gum-based $\mathrm{Ag}-\mathrm{TiO}_{2}$ composites treated at $300^{\circ} \mathrm{C}$ (c-e), and $500^{\circ} \mathrm{C}(\mathrm{f})$ and (g). Scale bars are $20 \mathrm{~nm}$ for (a), (c) and (f); $10 \mathrm{~nm}$ for (b), (d) and (g); and $5 \mathrm{~nm}$ for (e). Arrows indicate some of the Ag nanoparticles. Dashed ovals depict Ag nanoparticle rich areas. Dashed squares indicate enlarged regions in (e), and (g)). 91

Figure 71. TEM images of the primary $\mathrm{TiO}_{2}$ particles. Scale bars in (a) and (b) correspond to 20 and $10 \mathrm{~nm}$ respectively.

Figure 72. X-ray photoelectron spectroscopy detailed peaks $\mathrm{Ag} 3 \mathrm{~d}, \mathrm{Ti} 2 \mathrm{p}, \mathrm{C}$ 1s and $\mathrm{O} 1 \mathrm{~s}$, for the oil-based Ag-decorated $\mathrm{TiO}_{2}$ foam composites.

Figure 73. X-ray photoelectron spectroscopy detailed peaks $\mathrm{Ag} 3 \mathrm{~d}$, Ti $2 \mathrm{p}, \mathrm{C}$ 1s and $\mathrm{O} 1 \mathrm{~s}$, for the xanthan gum-based Ag-decorated $\mathrm{TiO}_{2}$ ink composites. 94 


\section{Table of Tables}

Table 1. Summary of ink-based AM direct printing techniques. ........................................................ 8

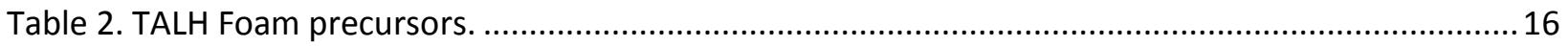

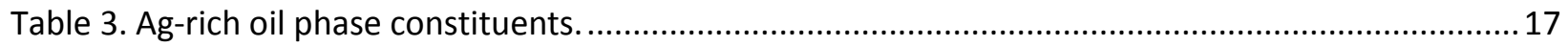

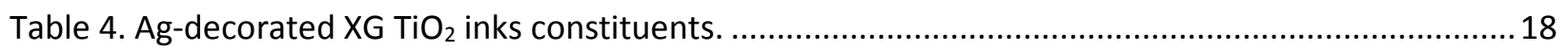

Table 5. Surface particle size, and Ag grain size dependence on annealing temperature......................27

Table 6. Yield stress and Herschel-Bulkley parameters for the studied AZO sol-gel inks.........................30

Table 7. lattice parameters for the $A Z O\left(\mathrm{Al}_{0.016} \mathrm{Zn}_{0.984} \mathrm{O}\right)$ structures, from inks with different PVP content in

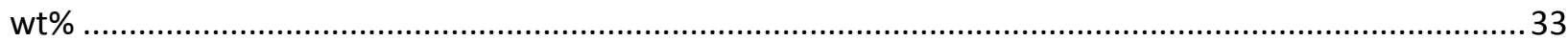

Table 8. Lattice parameters for the $\mathrm{AZO}\left(\mathrm{Al}_{0.016} \mathrm{Zn}_{0.984} \mathrm{O}\right)$ structures from $15 \mathrm{wt} \%$ PVP inks at different

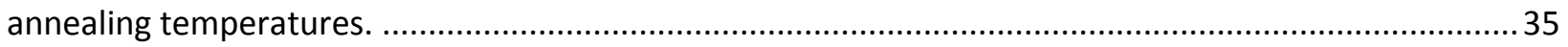

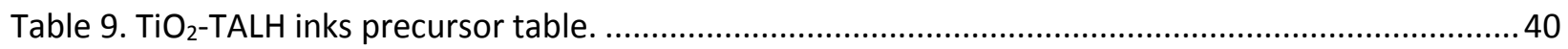

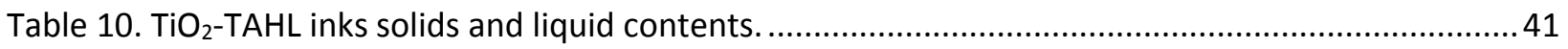

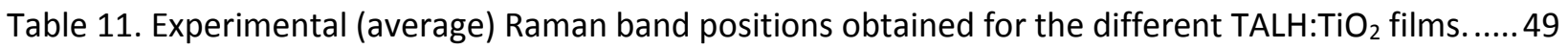

Table 12. Calculated amount of anatase and rutile (wt.\%) from the Raman and XRD data for the different

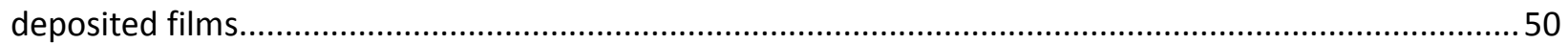

Table 13. Apparent first-order degradation rate constant $k_{a p p}$ and time for $50 \% \mathrm{MB}$ degradation from different $\mathrm{TiO}_{2}$ films. AFM and Profilometry roughness measurements for the films..............................55

Table 14. Film thickness measured by profilometry and electrical resistivity of the films as calculated from

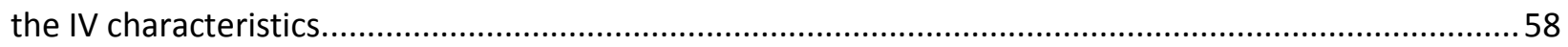

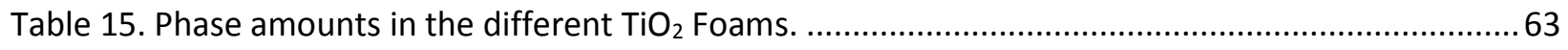

Table 16. Herschel-Bulkley coefficients for the 8 min frothed foams. ................................................65

Table 17. $\mathrm{TiO}_{2}$ sources in wt\% present in the wet foams, the targeted sintered structures (theoretical), and

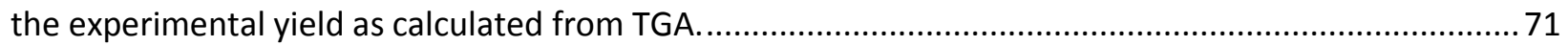

Table 18. Apparent first-order degradation rate constant $k_{a p p}$ and Brunauer-Emmett-Teller (BET) surface area.*Amount of liquid-solid-oil (L-S-O) in vol\%. .73 
Table 19. Amount of anatase and rutile phases, and crystallite size estimations from XRD data using Gaussian peak fits. The amount of liquid-solid-oil (L-S-O) is in vol\%. (1:6) refers to $\mathrm{TALH}_{\mathrm{TiO}} \mathrm{T}_{2}$ mole ratio. *alculated using the model by Gribb et al[208].

Table 20. Calculated lattice parameters for the $\mathrm{TiO}_{2}$ primary particles, and the resulting $\mathrm{TiO}_{2}$ in the studied foam systems. The amounts of liquid-solid-oil (L-S-O) are given in vol\%; (1:6) refers to TALH:TiO ${ }_{2}$ mole ratio. 76

Table 21. C:Ti and $\mathrm{Ag}: \mathrm{Ti}$ atomic \% ratios from the EDS spectra for the different energy treated $\mathrm{Ag}$ decorated $\mathrm{TiO}_{2}$ composites.

Table 22. Optical band gap $\left(\mathrm{E}_{\mathrm{g}}\right)$ of Ag-decorated $\mathrm{TiO}_{2}$ composites, and conduction band edge $\left(\mathrm{E}_{\mathrm{cb}}\right)$ of decorating Ag particles. ${ }^{*}$ Calculated from Tauc plots (linear region extrapolation). ${ }^{* *}$ Calculated using the Einstein's photon energy equation.

Table 23. X-ray photoelectron spectroscopy binding energy and modified Auger parameters AP for the $\mathrm{Ag}_{3 / 2}$ peak from the Ag-decorated $\mathrm{TiO}_{2}$ composites. .95

Table 24. Quantitative analysis of the sample composition, as calculated from the XPS peak fittings.....95 


\section{Introduction}

Additive manufacturing (AM) brings the advantages of highly controllable fabrication of printed features while allowing for the convergence of low-waste, lithography-free, tailorable microstructuring, potential for vertically-integrated device architectures fabrication, and large-scale manufacturability.

There is currently a great need to expand the library of available functional materials for use in additive manufacturing technologies. Therefore, AM of novel device architectures, relies on the development of materials with suitable processing and functional properties. Also, the understanding of the chemical and physical dynamics present in the solution synthesis of materials is of paramount importance when designing functional ink systems for printing. The inks undergo many processes that affect their properties. As these fluid systems are printed, dried, and cured/annealed/sintered their microstructure and composition change, favoring the crystallization of otherwise amorphous compounds, and the nucleation of secondary phase materials.

By studying the chemical and physical interactions, different ink systems can be designed to take advantage of such interactions and obtain specific microstructures, secondary phase features and or functional properties.

Therefore, control of the materials' microstructure using processing, becomes important for specific applications. Here, the strategies to control the microstructure using AM are explored for different metal and metal-oxide ink systems - namely, $\mathrm{Ag}, \mathrm{ZnO}$, and $\mathrm{TiO}_{2}$ based inks - from particle based, sol-gel based, and hybrid (particle/organic sol) inks, respectively. These materials find application across diverse technological fields including energy, environmental, and biomedical. Moreover, the choice of these particular materials, for studying the specific processing approaches, considers the ample literature available for their study; and is expected to be transferable to similar metal and metal-oxide material systems.

From such base materials, different ink systems are proposed and realized with fundamental studies of chemical and physical interactions. The control of the printed microstructures ranges from their particle size, porosity, texturing, and morphology to composite heterostructures' realization. This work, rather than being exhaustive about the possible chemical and physical material interactions, focuses on the main properties relevant to AM for potential device applications. Special emphasis is placed on sustainability, aiming for scalable and industry transferable manufacturing. 


\section{Materials Systems}

\section{Ag Ink (Particle Based)}

The Ag ink system is a highly concentrated mixture of Ag particles dispersed in a cellulose solution. These particles are capped with polyacrylic acid (PAA), which serves as stabilization agent for the dispersion. Once the material is applied to a substrate, a mild heat treatment will induce further crystallization and coalescence of neighboring particles.
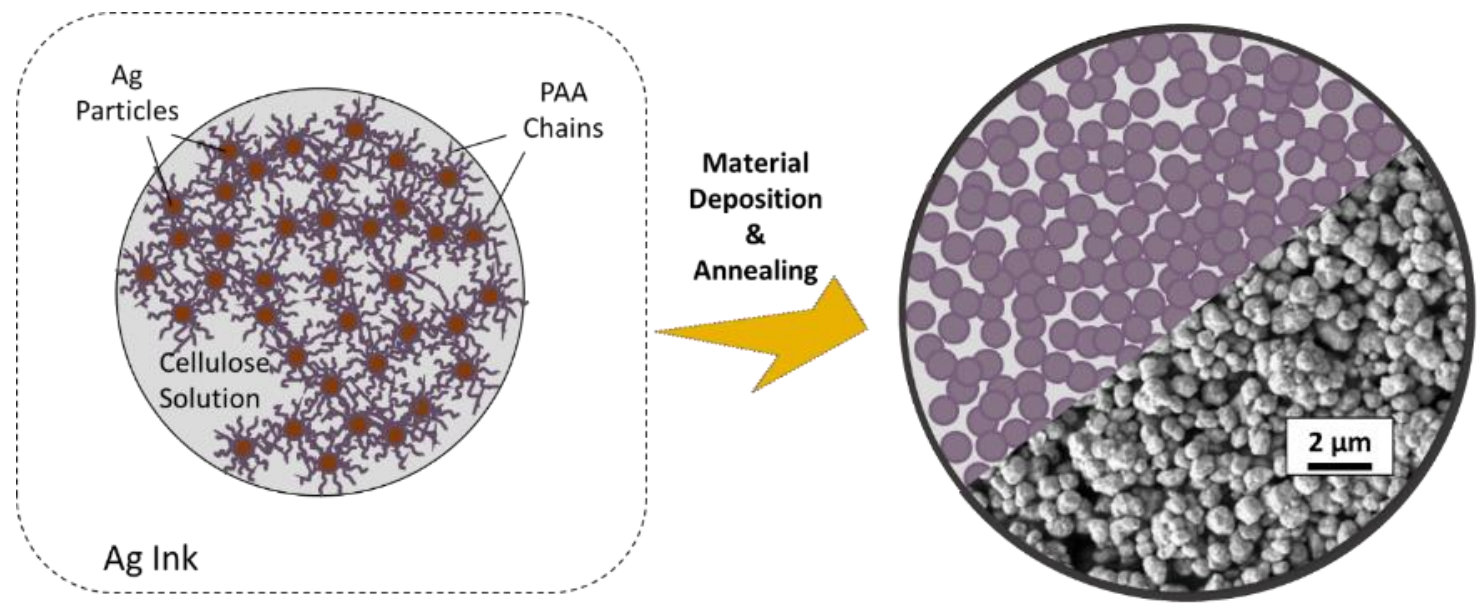

Figure 1. Ag particle ink system.

\section{Al-doped ZnO Ink (Solution Based)}

The Al-doped $\mathrm{ZnO}$ (AZO) sol-gel based ink, consists of a mixture of a $\mathrm{Zn}$ and $\mathrm{Al}$ organic precursors in solution using 2-methoxy ethanol as solvent, ethanolamine as stabilizer, and polyvinyl pyrrolidone (PVP) as rheology enhancer. This system is used to investigate relevant processing - properties relationships, particularly the microstructural evolution and texturing degree.

\section{$\mathrm{TiO}_{2}$ Hybrid Inks and Foams (Particle/Organic Sol Based)}

The $\mathrm{TiO}_{2}$-based materials proposed here implements a hybrid $\mathrm{TiO}_{2} / \mathrm{Ti}$-organic ink system. This combination includes the advantages of adding $\mathrm{TiO}_{2}$ in the desired crystalline phase for specific functionality (greatly reducing the energy input for post-processing), and the bottom-up characteristics by inducing and controlling the ink compounds' interactions during preparation, deposition and treatment. 


\section{$\mathrm{TiO}_{2}-\mathrm{TALH}$ Ink}

The $\mathrm{TiO}_{2}$ - $\mathrm{TALH}$ ink system consists of a crystalline phase and an amorphous phase. Ti(IV) bis(ammonium lactato) dihydroxide (TALH), is a water-compatible Ti-organic complex used as precursor for $\mathrm{TiO}_{2}$. After the ink deposition, such amorphous phase is transformed to a more ordered structure through mild heat treatments or by UV exposure utilizing the photocatalytic properties of $\mathrm{TiO}_{2}$.
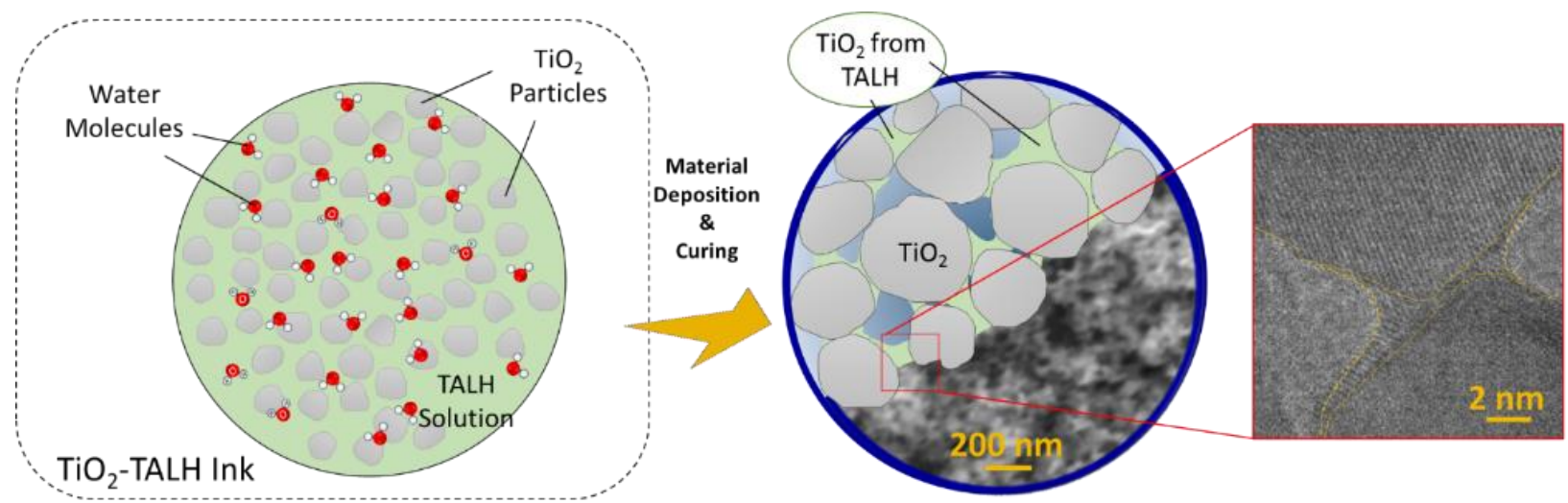

Figure 2. $\mathrm{TiO}_{2}$ - TALH ink system.

\section{$\mathrm{TiO}_{2}$-TALH Foam}

This material system, starts from the $\mathrm{TiO}_{2}-\mathrm{TALH}$ colloidal suspension as the aqueous phase in a multiphase emulsion system. An oil phase serves as a stabilization and emulsifier agent and, for the decorated structures, as encapsulating and dispersion agent for such molecules/materials. A third phase is included as air bubbles though frothing of the aqueous/oil emulsion rendering the desired foam systems.
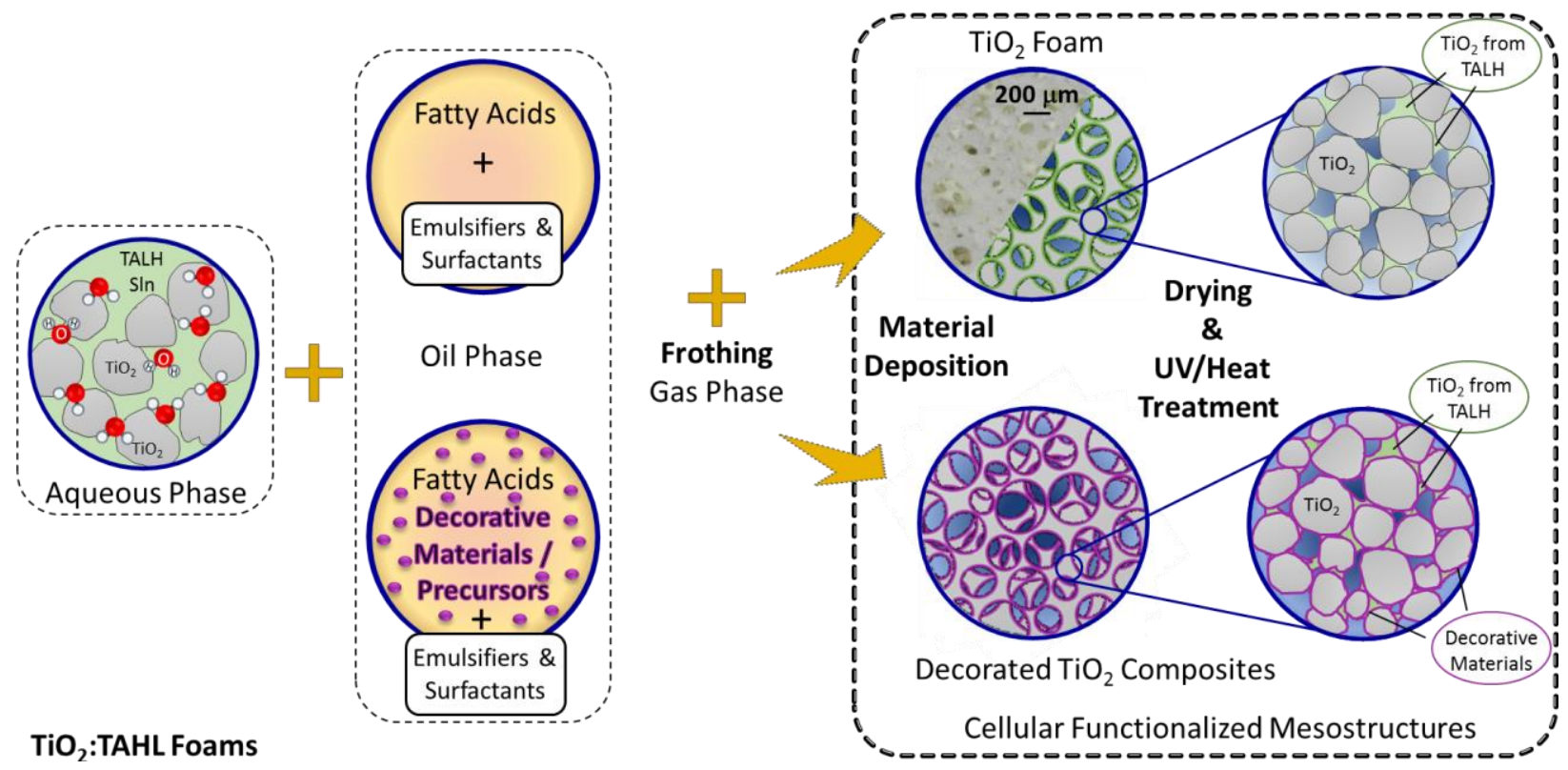

Figure 3. $\mathrm{TiO}_{2}$-TALH foams systems 


\section{Aims and Objectives}

Based on the potential of AM and the need for research on ink systems suitable for such processing, the following aims are set for this thesis:

Aims

1. To design metal ( $\mathrm{Ag}$ ) and metal-oxide (Al-doped $\mathrm{ZnO}$, and $\mathrm{TiO}_{2}$ ) material ink systems using a sustainability focused approach, to enable their industry transferability.

2. To establish and investigate fundamental relationships influencing the processing and properties of particle based, sol-gel and hybrid inks; exploring their potential for microstructure engineering.

3. To realize printable device components using the designed inks. Specifically, to design, synthesize and characterize $\mathrm{Ag}$ inks with potential application as electrically conducting structures and/or electro-mechanical sensors.

4. To design and study a composite $\mathrm{TiO}_{2}$ material controlling its surface area, porosity and crystallite interconnectivity. Furthermore, to study the relationships between the materials synthesis and the degree of crystallinity attained, and their electrical and surface chemistry properties.

5. To propose alternative means for the synthesis of secondary-phase decorated $\mathrm{TiO}_{2}$ composite heterostructures by incorporating the decorative materials and/or precursors as ink constituents, studying their transformation and allowing for one-step fabrication. This approach has the potential to produce novel microstructures, and to reduce the time associated to the decoration of metal-oxide scaffolds in applications such as sensitized solar cells, $\mathrm{H}_{2}$ generation, small molecule detection, and growth of biological tissues.

The objectives for the specific ink systems are outlined below:

\section{Objectives}

Ag (Particle) Based Inks

- To formulate Ag inks to be patterned though continuous-flow direct writing on flexible substrates and to study the relationships between synthesis, processing, and properties.

- To study the role and effects of different sol-gel constituents involved in the nucleation and growth of the Ag nanoparticles.

- To investigate the role and transformations of the different constituents of the formulated inks, such as primary materials (nanoparticles and crystallized materials), additives and thickening agents on the printing and upon energy input.

- To characterize the microstructure, optical and electrical properties of the printed systems. 


\section{Al-doped ZnO (Sol-Gel) Based Inks}

- To investigate the crystallization mechanisms of Al-doped ZnO (AZO) inks printed using continuous-flow direct writing, upon different post-printing energy inputs.

- To study the effects of different amounts of polymer additives as rheology enhancing agents on the processing and microstructure tuning of metal-oxide structures from sol-gel inks.

\section{$\mathrm{TiO}_{2}$ (Hybrid) and decorated Inks and Foams}

- To explore the viscosity ranges of the $\mathrm{TiO}_{2} / \mathrm{Ti}$-organic complex for their application using continuous-flow direct writing.

- To study the crystallization of $\mathrm{TiO}_{2}$ from the hybrid inks in contact with $\mathrm{TiO}_{2}$ crystals.

- To investigate the microstructure and functional properties of the proposed systems, and the effect of different UV and heat treatments.

- To study the role and transformations of different constituents of the formulated inks; i.e. primary materials (nanoparticles and crystallized materials), additives, thickening agents, surfactants (emulsifiers, stabilizers, etc.), and catalysts on the printing and upon energy input.

- To explore and propose alternative methods to introduce metal secondary-phase materials to the $\mathrm{TiO}_{2}$ scaffolds, and demonstrate their feasibility.

- To investigate the changes induced upon energy input on the Ag-decorated $\mathrm{TiO}_{2}$ colloidal materials. 


\subsection{Metal and Metal-Oxide Materials}

Metals and metal-oxides, represent an important group of materials with application across multiple technological fields[1-3]. These materials are commonly used in devices because of their functional properties; and specific mechanical, thermal, and chemical stability. Metals, are important as high electrical and thermally conductive contact materials[4-6], allowing for efficient electron and phonon transport, respectively. Additionally, metals and metal-oxides are often used in applications requiring relatively high mechanical stresses [7-12], as light scattering materials for reflective coatings[1], magnetic material applications[13,14], as catalysts[3,13,15-18], bactericidal agents[19,20], and many more. Specifically, metal-oxides are important due to their tunable optical and electrical properties[4,21], and high thermal and chemical stability $[17,22]$. They are generally non-toxic, abundant materials, which makes them good candidates for large scale applications.

These materials, can be synthesized in the nanoscale using top-down or bottom-up approaches, rendering outstanding properties that deviate from the typical behavior exhibited when in bulk[6,13,23-28]. Topdown methods are generally considered cost-effective and highly scalable; however, their compositional control and homogeneity may be compromised. Bottom-up approaches are preferred for high control of the chemical composition and microstructure[1]. Usually, a combination of manufacturing methods is used for the fabrication of devices incorporating techniques from both approaches[29]. Among the multiple bottom-up techniques, sol-gel is of interest because of its low cost, scalability, and wide range of precursors; leading to an equally broad range of materials' microstructures and morphologies[1,30-33]. Bottom-up synthesis, enables the fabrication of hierarchically structured mesoporous materials and device components[33-35]. Hierarchical structures are interesting material systems, because they combine features with dimensions spanning several orders of magnitude. Thus, they can be viewed as synergistic architectures that take advantage of the properties associated with materials at the nano, micro, meso and macro-scale[1,11,36-39]. Particularly, metal-oxide mesostructures of $\mathrm{TiO}_{2}$ have been prepared using hydrothermal processing [40-44], resembling hierarchically structured shapes such as flowers, trees, hollow spheres, etc.; chemical and physical vapor deposition of films $[41,45]$, and electrospinning of $\mathrm{TiO}_{2}$ fibers in mat-like configurations[46]. Similarly, examples of mesostructured metaloxides materials using sol-gel synthesis include $\mathrm{TiO}_{2}[33,47], \mathrm{MnO}_{2}[32], \mathrm{V}_{2} \mathrm{O}_{5}[32], \mathrm{CO}_{3} \mathrm{O}_{4}[48], \mathrm{ZnO}[32,49]$, $\mathrm{ZrO}_{2}[47], \mathrm{Al}_{2} \mathrm{O}_{3}$ [47], $\mathrm{WO}_{3}[32]$ and $\mathrm{SiO}_{2}[47,50]$. Moreover, composite materials consisting of metal/metaloxide structures exploit the interfacial effects for improved properties. These, can be prepared using a combination of processes; particularly, photoreduction of metal particles has been extensively used for the decoration of ceramic scaffolds and for studying the photocatalytic active sites/facets of the ceramic scaffold. Relevant material systems include $\mathrm{Ag}$ on $\mathrm{ZnO}[51,52]$, Pt on $\mathrm{WO}_{3}[53]$ and on $\mathrm{TiO}_{2}, \mathrm{Ag}$ on $\mathrm{TiO}_{2}[54,55]$ and multimetallic particles on $\mathrm{TiO}_{2}[56]$.

Generally, when using the sol-gel method, post-processing heat treatments are applied to induce the transformation of the amorphous materials into crystalline structures, and/or to promote their bonding, ensuring appropriate contact between the different materials and/or device layers. Importantly, lowtemperature based alternative routes have enabled the fabrication of devices from metal-oxides on heat 
sensitive substrates[1,7] towards large-area roll-to-roll manufacturing. These include solution based biomimetic synthesis[57-59], ultraviolet light curing[60,61], cold isostatic pressure sintering[62], combustion processing[63] and electrophoretic synthesis[45]. Remarkably, the use of hybrid approaches involving the combination of sol-gel metal-organic precursors and crystalline metal or metal-oxide particles have opened alternative routes for low temperature fabrication by reducing the thermal budget associated with the films transformation into higher crystallinity structures [60,64-66]. Less explored sintering/curing treatments include photonic sintering (provided that targeted light trapping materials are also included in the formulation and illumination matches their absorption spectrum); and electrical sintering, which has been demonstrated for conducting inks, namely Ag and indium tin oxide (ITO) $[67,68]$.

Currently, conventional micro-device manufacturing, relays in vacuum-based techniques such as e-beam evaporation and sputter coating[1]. The limitations associated to these techniques include large-scale manufacturing incompatibility, relatively high-cost and multi-step lithography patterning[69-72]. Traditional manufacturing methods associated to sol-gel processing such as spin coating, doctor blading (DB), and casting are not fully suited for large-scale pattern manufacturing[1,73]. Spin coating for example, may result in significantly different thick regions and film defects depending on the radial distance to the spinning center and the inks formulation. DB and casting on the other hand, still require the use of masks to produce the patterns. Direct patterning methods represent a novel and low-cost solution to overcome these challenges while enabling large-area substrate coverage, at the expense of surface finish quality and reduced electrical conductivity [4,74-82]. Other common manufacturing methods based on printing, such as screen-printing, are suitable for large-area processing; however some key challenges remain, including significant materials waste and yield of relatively large printed film thicknesses, potentially causing difficulty in controlling the final material thickness and morphology[71,83]. Additionally, the use of masks is often regarded as challenging since premature drying of the ink on the masks usually results in printing defects and loss of printing definition [83]. Maskless DW printing methods aid in mitigating the aforementioned issues with ink-jet printing being the most representative method [84-87]. Nevertheless, the exclusive utilization of low-viscosity inks used for ink-jet processes (usually ranging from $\sim 3-60$ cPoise) $[73,84,88]$, limits its one-step 3D printability and may result in uneven distribution of dried solute, also known as the coffee ring effect[89].

\subsection{Additive Manufacturing using Metal and Metal-Oxide Inks}

$A M$ is a comprehensive set of fabrication techniques that involve adding material to build up a component / device on a layer-by-layer basis. Briefly, these include stereo lithography (SLA), powder/slurry based 3D printing and laser sintering, laminated object modelling (LOM), fused deposition modelling (FDM), and direct writing ink based techniques: ink-jet printing, aerosol-jet writing, dip-pen nanolithography, and continuous-flow direct writing (CDW)[85,90-92]. AM techniques have gained interest from industry and academia due to their versatility and reduced waste capabilities by utilizing specific amounts of material where necessary[90]. Building a component layer-by-layer, allows controlling its structural properties by incorporating differential material properties based on the part's design and operational requirements, while reducing the component weight[85,92,93]. Direct writing (also known as additive printing), implements materials formulated as inks to pattern the desired device/component architectures[85,91]. Dip-pen nanolithography is used to pattern materials using a quill-based approach in which the inks are transferred to a substrate using an AFM tip, this technique enables features in the nanoscale and 
manipulation of materials with high precision. Aerosol-jet printing deposits inks that are suspended as an aerosol mist, which is delivered to the desired substrate utilizing a carrier gas, this technique allows features as small as $5 \mu \mathrm{m}$. Ink-jet printing utilizes a piezo-electric controller to generate droplets of a low viscosity ink that are deposited building a pattern by the coalescence of the droplets with resolution features as small as $\sim 20 \mu \mathrm{m}$; while CDW utilizes an extrusion system to pattern the ink as a continuous filament, with feature sizes spanning from $\sim 5 \mu \mathrm{m}$ to a few millimeters $[85,94]$. The latter is of great interest because of its versatility allowing for the fabrication of material structures with unprecedented characteristics[93,95-99] such as lightweight architectures, complex microstructures, high aspect ratio walls and spanning features, by combining the patterning/manufacturing with bottom-up material synthesis[85].

In CDW (Figure 4), the precursor material consists of functional flowable inks that are extruded to form features by patterning them at specific, digitally predefined, substrate locations[100]. This method, allows for 2D and 3D patterning on planar and arbitrary shaped surfaces, with lateral dimensions of up to two orders of magnitude lower than those achieved by ink-jet printing[94,101,102]. CDW employs a PCcontrolled $x-y-z$ stage that moves relative to a device (i.e. a dispensing nozzle) to pattern the ink[103]. This method has been introduced a few years ago[95] and has recently re-emerged due to its inherent ability to extrude a wide range of viscosities from $\sim 10^{2}$ to $10^{6} \mathrm{mPa} \cdot \mathrm{s}[93,104,105]$, thus enabling significant ink design freedom and fabrication of planar and 3D film/pattern architectures[93,106]. Depending on the ink initial properties, different post-printing treatments may be necessary to provide the part with better mechanical and chemical stability characteristics, and to promote specific material crystalline structures and surface properties. Therefore for CDW, the material being extruded imparts a strong dependence on the printed components, and together with the changes induced by the post-printing processing, are considered as being essential aspects for the successful fabrication of the component[107,108]. For example, alignment of the colloidal materials may be induced; and porosity, surface finish and dimensional changes will depend on ink properties, and curing/sintering treatments[93].

Table 1. Summary of ink-based AM direct printing techniques.

\begin{tabular}{ccccc}
\hline Technique & $\begin{array}{c}\text { Dip-Pen } \\
\text { Nanolithography } \\
\text { (DPN) }\end{array}$ & Aerosol-Jetting & Inkjet Printing & $\begin{array}{c}\text { Continuous-Flow } \\
\text { Direct Writing } \\
\text { (CDW) / } \\
\text { Robocasting }\end{array}$ \\
\hline $\begin{array}{c}\text { Deposition } \\
\text { Method }\end{array}$ & Quill-based & Stream jetting & Droplet jetting & Filament extrusion \\
Ink Viscosity & $10^{1}-10^{2} \mathrm{mPa} \cdot \mathrm{s}$ & $10^{0}-10^{3} \mathrm{mPa} \cdot \mathrm{s}$ & $10^{1}-10^{2} \mathrm{mPa} \cdot \mathrm{s}$ & $10^{2}-10^{6} \mathrm{mPa} \cdot \mathrm{s}$ \\
{$[109]$} & {$[110]$} & {$[65,73,88]$} & {$[85,93]$} \\
Printing & $\sim 5-14 \mathrm{~nm}$ & $\sim 5-10 \mu \mathrm{m}$ & $\sim 10-30 \mu \mathrm{m}$ & $\sim 5 \mu \mathrm{m}-2-3 \mathrm{~mm}$ \\
Resolution & {$[85,98]$} & {$[85]$} & {$[93]$} & {$[93,94]$} \\
Printing & OD $-1 \mathrm{D}$ & $1 \mathrm{D}-2 \mathrm{D}$ & $2 \mathrm{D}-3 \mathrm{D}(\mathrm{limited})$ & $2 \mathrm{D}-3 \mathrm{D}$ \\
Capability & & & & \\
\hline
\end{tabular}




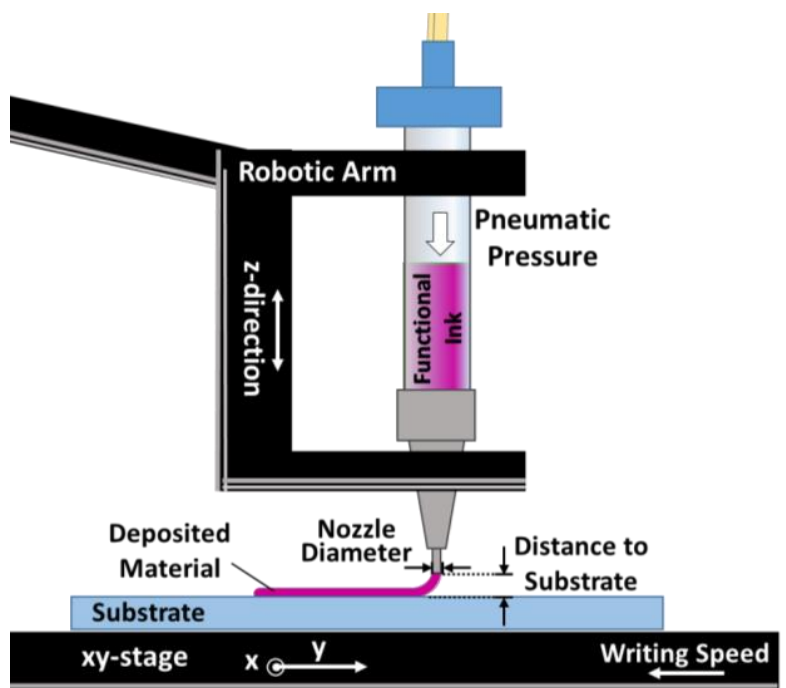

Figure 4. Continuous-flow direct writing technique schematic. Distance to substrate is enlarged for visualization purposes.

Device fabrication often requires the combination of different printing techniques including CDW and others such as ink-jet or screen printing[29,111,112]; with the latter exhibiting comparatively large material waste during the process[72]. Other relevant printing techniques used for the AM of devices include gravure printing, slot-die coating, and DB. The choice of printing technique is generally based on the inks viscosities[71-73,113,114], the different resolution capabilities, and the specific device architectures $[29,71,73,83]$. Studies integrating the different manufacturing techniques are important because of their industry-transferable character, and because they serve as pioneering pathways towards fully printable devices using AM. To date, examples of fully CDW fabricated device prototypes are Li-ion microbatteries[115,116], a light emitting diode[117], a bionic ear[118], and thick-film carbon based supercapacitors[119,120].

The functional inks may involve the exclusive use of particle-free sol-gel formulations, or include particulate materials dispersed as colloidal systems. Inks of different materials have been investigated, producing microstructured materials. Relevant metal and metal-oxide systems include $\mathrm{Ag}[94,121-123]$, $\mathrm{TiO}_{2}$ [124-128], $\mathrm{ZnO}\left[129-131\right.$ ], ITO [132], $\mathrm{Al}_{2} \mathrm{O}_{3}$ [133-135], $\mathrm{SiO}_{2}$ blends[107], PZT[136] and C-based materials[137-139].

Among the most relevant properties for successful CDW are the rheological, viscoelastic and drying properties of the inks[140,141]. These determine the printing ranges and the characteristic feature dimensions [107]. Tuning of the viscosity may be accomplished by using polymeric agents and by modifying the solids amount in the inks[101,108,142]. Additionally, the use of polymeric additives as rheology enhancers can be used to reduce nozzle clogging issues common to nozzle-based printing methods due to ink solids sedimentation[128]. The dimensions of the final CDW features are also dependent on the nozzle shape[143] and diameter, applied extrusion pressure, and printing speed[121,131,132,139,144].

When using CDW, the inks should display a shear-thinning behavior, that allows them to be extruded at relatively low pressures[145]. At the same time, they should recover upon stress release to retain their 
printed shape. This may be accomplished by engineering the inks using colloidal dispersions[101,146] and viscosity enhancer agents[107,121,124,128]; and/or fast solvent evaporation, coagulating or hydrolyzing sol-gel formulations[124,147].

Such viscous ink formulations exhibit rheological characteristics that can be approximated to the HerschelBulkley model as indicated in Eq. 1

$$
\tau=\tau_{y}+k \dot{\gamma}^{n}
$$

Where, $\tau$ is the total shear stress and $\tau_{\mathrm{y}}$ the fluid yield-stress; $\mathrm{k}$ denotes the fluid consistency, and $\mathrm{n}$ is the power law index accounting for the flow characteristics [148]. For Newtonian fluids $n=1$ and ty=0; if $\tau_{y}>0$ then the fluid is known as Bingham plastic. When $0<n<1$, then the fluid is classified as shear-thinning; and for $n>1$, it is classified as shear thickening.[149-151] Yield stress fluids, also known as Bingham fluids, exhibits solid-like behavior when no external stress is applied, and liquid-like characteristics when subjected to shear stresses above the yield stress, therefore they are ideal for CDW. The yield stress $\tau_{y}$ can be calculated from the extrapolation of the linear fit of the shear-stress vs. shear-rate curves, at the intercept of such fit with the shear-stress axis[150,151].

\subsection{Printing Cellular Architectures using CDW}

Additional to the different levels of structuring of CDW solid bodies that can be realized by the design of 2D and 3D patterns[93,152], highly mesoporous ceramics and cellular architectures are currently at the spotlight of research using CDW[10,99,152-156]. Ceramic based foams are highly desirable material systems because of their ability to mimic hierarchical organization widely existing in biological organisms[145]. Such organization is beneficial in numerous applications from catalysis[36,157-159] to energy harvesting[39,160-162] and storage[163], to biomedical[164-166].

Despite the early studies demonstration of advantageous mechanical properties of $\mathrm{Al}_{2} \mathrm{O}_{3}$ cellular structures $[10,154]$ produced using CDW, the employed ink synthesis methods utilize relatively large amounts of acid reagents to stabilize the particles and concomitantly gas bubbles forming the pores of the system, which represents challenges for their safe manipulation and large-scale implementation. Furthermore, such studies report exclusively closed-cell foam architectures, limiting the range of synthesized materials. Direct foaming[167] is considered the most promising fabrication route for ceramic foam 3D printing (among the established synthesis methods: replica, sacrificial template and directfoaming)[168], because of the ability to control viscosity and prepare the foam as a patternable extrudate, while producing different microstructure and porosity configurations (i.e. open- or closed-cell architectures)[2].

Some of the challenges associated to the conventional synthesis of ceramic foams include the repetitive impregnation (or incorporation) and calcination removal of organic templates[11,157]; or the time sensitive handling of rapid-hydrolyzing of liquid-liquid[169,170] and gas-liquid[171] emulsions systems. More recent methods, include the decomposition reaction of $\mathrm{TiCl}_{4}$ within aqueous-organic solvent mixtures, in which the pores are generated by $\mathrm{HCl}$ toxic fumes as a by-product of the hydrolysis of the Tiprecursor[162]. Developments on the colloidal processing of hierarchical mesoporous cellular ceramics are of great interest because of the versatility that colloidal science brings to manufacturing. 
Thus, CDW of metal and metal-oxide materials, offers new opportunities for device manufacturing; as well as new routes for the engineering of materials, and the study of their fundamentals, by enabling new material architectures and by providing new models for their processing.

\subsection{Sustainability Based Additive Manufacturing}

With greater consciousness of a growing global population and the limited resources, the concepts of "Green Chemistry" and "Green Engineering" are becoming increasingly important for the realization and adoption of new technologies[172]. Sustainability is therefore, a central issue that can be addressed from various ends regarding additive manufacturing[173]. Specifically, the observation of the 12 principles of green chemistry[174] and green engineering[175], and the life cycle analysis of products and precursors constitutes a primary step towards responsible and industry transferable engineering[176,177]. Therefore, the adoption of metrics and assessment applied to the specific processes, should be specially considered due to their high impact in emerging manufacturing fields[173]; since the incorporation of such concepts in the assessment of novel manufacturing developments ensures their feasibility, and the health and safety of the environment and the individuals involved.

Additive manufacturing, starts its sustainability focus by pursuing the optimization of the materials and energy involved in the fabrication of components fulfilling the first and second principles of green chemistry: waste-prevention, and maximization of the use of precursor materials into the final products, respectively. The third principle refers to the use of innocuous materials and substances whenever possible; AM using aqueous based formulations, helps preventing the massive use of organic solvents, commonly associated with harmful effects to human health and to the environment. Along the same line, the fourth principle, seeks for the designing of safer chemicals and materials. The fifth principle, is the reduction in the use of solvents and auxiliary substances in the processing, and the use of innocuous ones when necessary. AM is also advantageous from this perspective, since it reduces their use by preventing the need of etching and cleaning between processing steps. The sixth and seventh principles are the implementation of processing methods at ambient temperature and pressure, and the use of renewable rather than depleting resources, respectively. This principle not only aims for the better use of the available resources, but it also pushes for new advances in the realization of materials and devices using soft materials and offering unexplored applications. Next, the eight principle focuses on the minimization of derivatization, as this is likely to result in extra sources of waste. Because AM offers new routes for the microstructuring of materials, this principle may also be applied. The preferential use of catalytic reagents when compared to stoichiometric ones, constitutes the ninth principle, which applied to AM is expected to be of clear benefit by enabling more efficient post-printing processes. Finally, principles tenth to twelfth, refer to the design of materials considering their degradation, the assessment of the environmental impact and potential risks, and the use of the safest substances and substance forms as to minimize their harmful effects in case of chemical accidents, such as release to the environment, fires and explosions; taking into account the central safety aspects, AM being highly automated, may be considered as an inherently safer approach than traditionally fabrication processes. Thus, research on new materials incorporating these principles for AM in general, and CDW in particular, is imperative for furthering of the progress of large-scale device manufacturing. 


\section{Chapter 2: Experimental Details}

\subsection{Inks Synthesis}

\subsubsection{Ag Ink}

For the synthesis of $\mathrm{Ag}$ nanoparticles, a base-aided reduction of $\mathrm{AgNO}_{3}$ was used for the metallic $\mathrm{Ag}$ nucleation. A modification of a previously reported solution-based method[121] including $\mathrm{AgNO}_{3}, \mathrm{PAA}$ and mono-ethanolamine (MEA) mixed in water is implemented, in an aim to increase the Ag yield. See Figure 5.

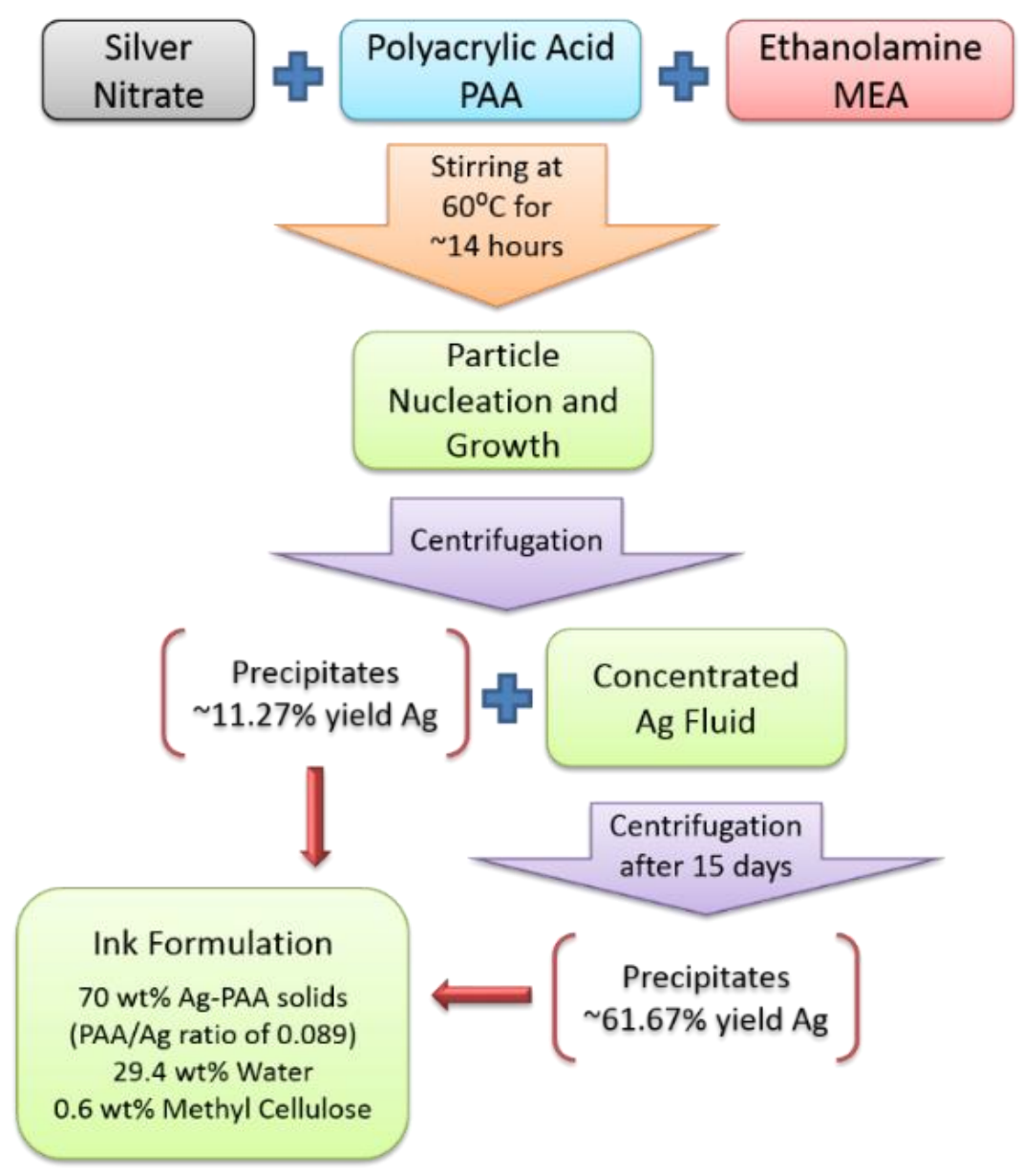

Figure 5. Ag- Ink synthesis process and precursor chemicals.

Briefly, PAA is dissolved in half the amount of water and magnetically stirred until homogeneous, and the MEA is added dropwise to the stirring PAA-water solution. In parallel, the $\mathrm{AgNO}_{3}$ is dissolved in the remaining water and dropwise incorporated to the PAA solution. The amounts of reducing agent (MEA) with respect to the solvent, $\mathrm{Ag}$ precursor and capping agent (PAA), affect the nucleation and growth process as is discussed in chapter 3. For the nucleation of the Ag nanoparticles and formulation of inks used in the patterned structures, investigated in such chapter, final precursor amounts are: $0.57 \mathrm{~g}$ PAA, $10.19 \mathrm{~g} \mathrm{AgNO}_{3}, 8.9 \mathrm{~g} \mathrm{MEA}$ and $35.9 \mathrm{~g} \mathrm{DI}$ water. This solution is then stirred for a few hours ( $\left.14-24 \mathrm{~h}\right)$ to 
allow for particle nucleation and growth. Temperature $\left(\sim 60^{\circ} \mathrm{C}\right)$ may accompany the process to accelerate it. The resulting colloidal suspension is brown-black in color. Once the solution is at room temperature ethanol is added to help the coagulation of the Ag particles. Since it is a poor solvent for the PAA, the supernatant is removed and some more ethanol is added to transfer the sediment-rich solution to a centrifuge tube. After centrifugation at $4400 \mathrm{rpm}$ for 30 minutes, the Ag nanoparticles are collected and the inks are formulated. The Ag solids are re-dispersed in half the final amount of solvent (water) by ultrasonication, and latter a water-cellulose solution is added, further sonicated, and mixed until a homogenous ink is obtained.

\subsubsection{Al-Doped ZnO (AZO) Ink}

The Al-doped $\mathrm{ZnO}$ sol-gel system is a variation of the sol-gel ink used by O'Brien et.al.[178]. For its synthesis, a precursor mixture containing $3 \mathrm{wt} \%$ of aluminum nitrate $\left(\mathrm{Al}\left(\mathrm{NO}_{3}\right)_{3}\right)$ and $97 \mathrm{wt} \%$ zinc acetate dehydrate $\left(\mathrm{Zn}\left(\mathrm{CH}_{3} \mathrm{COO}\right)_{2} 2 \mathrm{H}_{2} \mathrm{O}\right)$ is prepared in $16 \mathrm{~mL}$ of 2-methoxy ethanol, and $2 \mathrm{~g}$ of ethanolamine (stabilizer), yielding a stabilizer to precursor molar ratio of 2 . This mixture is vigorously stirred for 2 hours with a magnetic stirrer (Fisher Scientific Isotemp) at $60{ }^{\circ} \mathrm{C}$ to aid dissolution of the solid reagents. The solution is, thereafter allowed to age at room temperature for 24 hours. PVP Mw 1,300,000 is added as ink-thickening agent in 10, 15 and 20 wt\% of the AZO-precursor solution. Further stirring for 24 hours at $60^{\circ} \mathrm{C}$ is performed to ensure the homogenization of the PVP added mixtures, and to induce gelation of the inks. Degassing of the sol-gels is achieved by allowing the inks to settle for at least 24 hours.

\subsection{2. $\mathrm{TiO}_{2}$ - TIAA Ink}

Investigation of hybrid $\mathrm{TiO}_{2}$ / Ti-organic formulations using $\mathrm{Ti}$ diisopropoxide bis(acetylacetonate) (TIAA) was performed as preliminary studies to assess their feasibility, such discussion is included as Appendix B.

For the $\mathrm{TiO}_{2}$-TIAA ink system, a variation of a solution based TIAA ink system[124,125] was implemented by the incorporation of $\mathrm{TiO}_{2}$ crystalline nanoparticles to obtain a hybrid (organic-inorganic) Ti-based system. PVP Mw 8000 - Acros Organics, or PAA Product \#323667 - Sigma Aldrich, and a third of the total ethanol are mixed in a glovebox in $\mathrm{N}_{2}$ atmosphere. Once solubilized, the Titanium IV diisopropoxide bis(acetyl acetonate) (TIAA) is added and stirred until homogenous. Separately, $\sim 10 \%$ of the remaining ethanol, the DI water and ammonium hydroxide are vortex-mixed. In parallel, the $\mathrm{TiO}_{2}$ particles (Titanium (IV) oxide $\mathrm{TiO}_{2}$ nanopowder ( $21 \mathrm{~nm}$ diameter) Aeroxide ${ }^{\circledR}$ P25 (70\% Anatase, 30\% Rutile) - Sigma Aldrich (when adding crystalline $\mathrm{TiO}_{2}$ phase), and the rest of ethanol are mixed by sonicating (for $\sim 1 \mathrm{~min}$ ) and stirring (for $5 \mathrm{~min}$ ) twice in an alternating pattern, to thoroughly disperse the particles. $\mathrm{The}^{\mathrm{TiO}} \mathrm{O}_{2}$ mixture is dropwise incorporated to the TIAA solution while stirring until homogeneous. Then, the ethanol/water/ammonia solution is also incorporated in a dropwise manner and kept closed until homogeneous. The temperature is raised to $\sim 60^{\circ} \mathrm{C}$ and left stirring for $\sim 17$ hours in an open vial to allow for the solvent to evaporate thickening the ink. The different precursor ratios are $\left(\mathrm{TiO}_{2}\right.$ variable : Polymer 1 : Ethanol 48.6 : DI water 18.5 : Ammonium hydroxide 1.8 : TIAA 4.6) in mol.

\subsection{3. $\mathrm{TiO}_{2}$-TALH Ink}

The use of water-compatible Ti-organic precursors such as TALH, allows better manipulation of the inks, and aids preventing undesired issues such as clogging of the printing systems, as discussed in chapter 5, 6 and Appendix B. 
For the synthesis of this ink system, appropriate amounts of TAHL solution ( 50 wt\% in $\mathrm{H}_{2} \mathrm{O}$ ) - Sigma Aldrich, DI water and $\mathrm{TiO}_{2}$ nanoparticles (Aeroxide ${ }^{\circledR}$ P25)- Sigma Aldrich, were mixed and set to stir for about $10 \mathrm{~min}$. Then, the inks were sonicated in a water/ice bath for 15 minutes while occasionally stirring to prevent sedimentation.

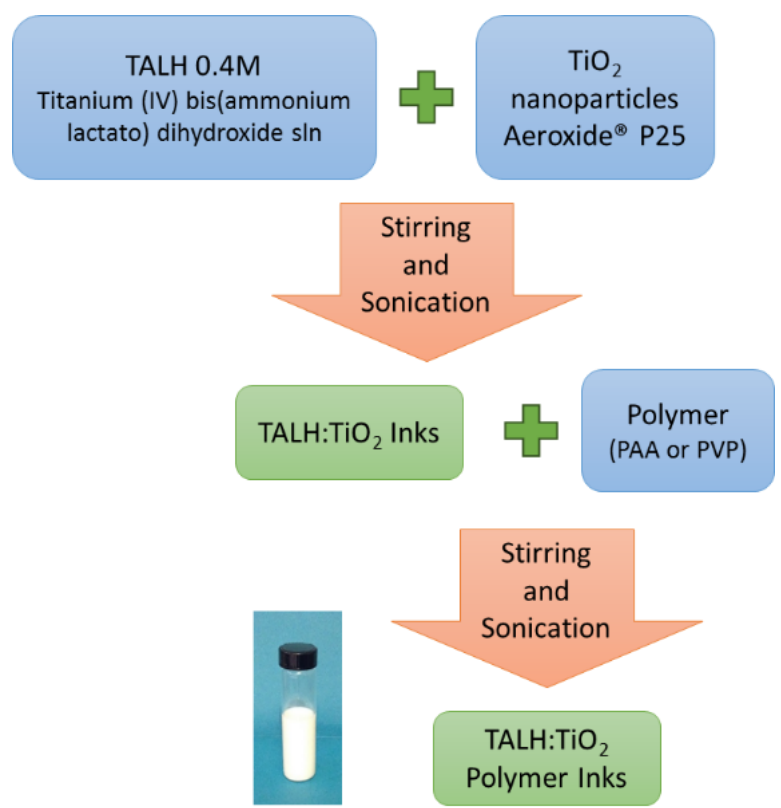

Figure 6. Schematic of the synthesis process for the TALH:TiO 2 inks and picture of a vial containing one of the formulated inks.

The TALH concentration was kept to $0.4 \mathrm{M}$ for all formulations, whereas the crystalline $\mathrm{TiO}_{2}$ phase was varied in $\mathrm{TALH}: \mathrm{TiO}_{2}$ molar ratios of (1:1, 1:3, 1:6 and 1:12). In parallel, DI water and PAA Product \#323667 - Sigma Aldrich or PVP Mw 8000 - Acros Organics, were mixed and stirred until becoming homogeneous with a magnetic stirrer. The TAHL:Polymer molar ratio was kept equal to 1 . The polymer solutions were dropwise added to the titania solutions while stirring and set for sonication for 15 more minutes. The obtained inks were stored in the same vials used for preparation and were magnetically stirred right before deposition.

\subsection{4. $\mathrm{TiO}_{2}-\mathrm{TALH}-\mathrm{FOam}$}

To synthesize the foam, the crystalline $\mathrm{TiO}_{2}$ particles are incorporated to the TALH and water solution to form the aqueous phase, then the PAA solution is incorporated, and the mixture is sonicated and stirred to ensure good dispersion. In parallel, the oil phase constituents: stearic acid (SA), polyoxoethylene sorbitan monostearate ( $\mathrm{P}-60$ ) and lanolin are mixed in the appropriate ratios and allowed to homogenize at $\sim 70^{\circ} \mathrm{C}$. Once homogeneous, the aqueous colloidal system is dropwise added to the oil phase while stirring. The temperature is kept to $70^{\circ} \mathrm{C}$ while blending these two systems, that are slowly allowed to cool by continuously stirring after the heat is stopped once the mixture is homogeneous. Finally, the ethanolamine is dropwise added to the resulting emulsion and allowed to stir until homogeneous. At this point the air bubbles are incorporated by frothing the mixture with an electric mixer. 


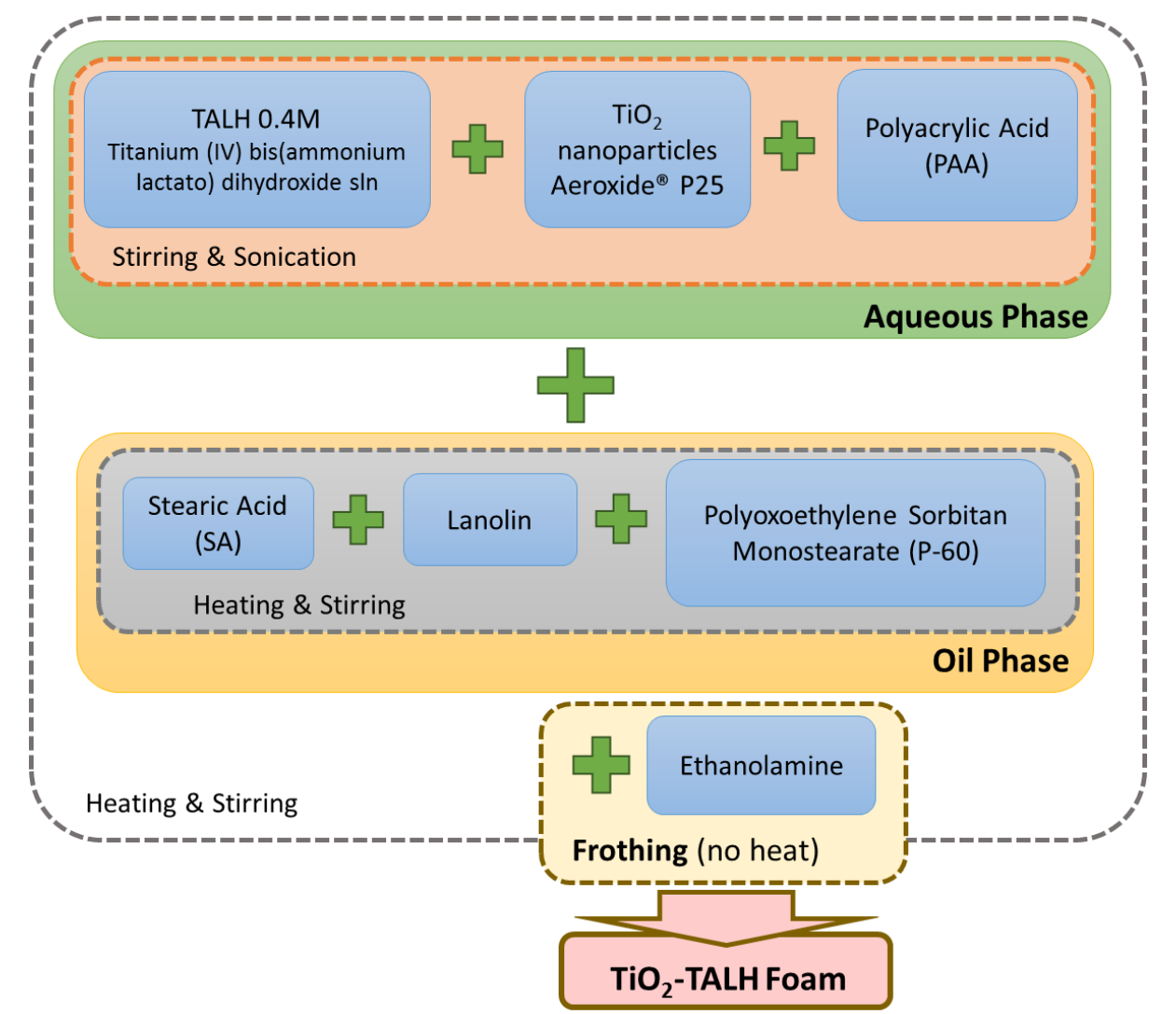

Figure 7. Schematic synthesis process for the TALH:TiO 2 foams.

The resulting emulsion increases its volume and flows readily through different diameter nozzles. After the foam structures have dried by allowing them to evacuate the solvent while left undisturbed and without additional heat of ventilation sources, thermal annealing and/or UV treatments are performed in order to transform the TALH into $\mathrm{TiO}_{2}$ around the existing $\mathrm{TiO}_{2}$ particles forming the $\mathrm{TiO}_{2}$ open-cell foam, and to decompose the oil phase constituents.

The nominal composition of the foams is: aqueous phase (in mol ratio): (TALH 1 : $\mathrm{TiO}_{2}$ variable : PAA 1 : DI water variable); oil phase ratios (including MEA or TEA, in wt\%): (SA 33.44 : P60 33.39 : Lanolin 27.84 : MEA/TEA 8.34); the mole ratio of SA to TALH is 2 and 1 for the L75-S3-O22 and L75-S5.5-019.5 formulations, respectively. The L-S-O notation is used to indicate the foams liquid-solid-oil volume ratio, these may be varied. 
Table 2. TALH Foam precursors.

\begin{tabular}{|c|c|c|c|c|c|}
\hline Precursor & Function & $\begin{array}{l}\text { Chemical } \\
\text { Formula }\end{array}$ & Chemical Structure & $\begin{array}{c}\text { Molecular } \\
\text { Weight } \\
\text { (g/mol) }\end{array}$ & $\begin{array}{c}\text { Concentration } \\
\text { (mol) }\end{array}$ \\
\hline DI Water & Solvent & $\mathrm{H}_{2} \mathrm{O}$ & & 18.015 & Variable \\
\hline $\begin{array}{l}\text { Titanium } \\
\text { bis(ammonium } \\
\text { lactato) } \\
\text { dihydroxide - } \\
\text { TALH }\end{array}$ & $\begin{array}{c}\text { Ti-organic } \\
\text { (precursor for } \mathrm{TiO}_{2} \\
\text { bridging } \\
\text { structures) }\end{array}$ & $\begin{array}{c}{\left[\mathrm{CH}_{3} \mathrm{CH}(\mathrm{O}-\right.} \\
\left.\mathrm{CO}_{2} \mathrm{NH}_{4}\right]_{2} \mathrm{Ti}(\mathrm{OH})_{2}\end{array}$ & $2 \mathrm{NH}_{4}^{+}$ & 294.08 & 1 \\
\hline $\begin{array}{l}\text { Titanium } \\
\text { Dioxide } \\
\text { Nanoparticles } \\
\text { (20 nm } \\
\text { diameter) - } \\
\text { Aeroxide }{ }^{\circledR}\end{array}$ & $\begin{array}{c}\text { Primary particles } \\
\text { (target material } \\
\text { composition) }\end{array}$ & $\mathrm{TiO}_{2}$ & & 79.865 & Variable \\
\hline $\begin{array}{l}\text { Polyacrylic Acid } \\
\text { - PAA }\end{array}$ & $\begin{array}{c}\text { Adhesion } \\
\text { promoting } \\
\text { Nozzle-clogging } \\
\text { preventing }\end{array}$ & $\left(\mathrm{C}_{3} \mathrm{H}_{4} \mathrm{O}_{2}\right)_{\mathrm{n}}$ & & 72.033 & 1 \\
\hline Stearic Acid & $\begin{array}{c}\text { Oil phase } \\
\text { constituent }\end{array}$ & $\mathrm{CH}_{3}\left(\mathrm{CH}_{2}\right)_{16} \mathrm{COOH}$ & $\mathrm{CH}_{3}\left(\mathrm{CH}_{2}\right.$ & 284.304 & $\begin{array}{c}1-2 \mathrm{~mol} / \\
\text { Oil-phase } \\
\text { (33.44 wt\%) }\end{array}$ \\
\hline Lanolin & $\begin{array}{c}\text { Oil phase } \\
\text { constituent } \\
\text { Emulsifier }\end{array}$ & ---- & & ---- & $\begin{array}{c}\text { Oil-phase } \\
\text { (27.84 wt\%) }\end{array}$ \\
\hline $\begin{array}{l}\text { Polyoxoethylene } \\
\text { Sorbitan } \\
\text { Monostearate }\end{array}$ & $\begin{array}{l}\text { Oil phase } \\
\text { constituent } \\
\text { Surfactant } \\
\text { Emulsifier }\end{array}$ & $\begin{array}{c}\mathrm{C}_{64} \mathrm{H}_{126} \mathrm{O}_{26} \\
\mathrm{x}+\mathrm{y}+\mathrm{z}+\mathrm{w}=20\end{array}$ & & 1311.046 & $\begin{array}{l}\text { Oil-phase } \\
\text { (33.39 wt\%) }\end{array}$ \\
\hline Ethanolamine & $\begin{array}{c}\text { Oil phase } \\
\text { constituent }\end{array}$ & $\mathrm{NH}_{2} \mathrm{CH}_{2} \mathrm{CH}_{2} \mathrm{OH}$ & & 61.064 & Oil-phase \\
\hline Triethanolamine & $\begin{array}{l}\text { Surfactant } \\
\text { Emulsifier }\end{array}$ & $\mathrm{C}_{6} \mathrm{H}_{15} \mathrm{NO}_{3}$ & & 149.130 & (8.34 wt\%) \\
\hline
\end{tabular}




\subsubsection{Ag-decorated $\mathrm{TiO}_{2}$ Inks and Foams}

The synthesis of the Ag-decorated $\mathrm{TiO}_{2}$ inks/foams uses the $\mathrm{TiO}_{2}: \mathrm{TALH}$ system as mixture precursor. The incorporation of $\mathrm{Ag}$ is performed via the encapsulation of solubilized $\mathrm{Ag}$-acetate in a second phase liquid solution and its subsequent blend with the $\mathrm{TiO}_{2}: \mathrm{TALH}$ mixture. Two systems are described based on the kind of encapsulation medium used (i.e., oil-based vs. xanthan gum (XG) aqueous-based). These different encapsulation media are used because to assess their potential used, and since their intrinsic differences are expected to affect the decorative materials formation.

\section{Ag-Decorated $\mathrm{TiO}_{2}$ Foam (Oil-based)}

To prepare the oil-based Ag-decorated foams, the oil phase constituents (SA, P-60 and lanolin) are mixed and stirred until homogeneous at $\sim 70^{\circ} \mathrm{C}$. The Ag-acetate solution (Ag-acetate in ethanol, and the corresponding ethanolamine or triethanolamine) is added to this mixture and allowed to homogenize, afterwards the solvent is allowed to evaporate while stirring. In parallel, the $\mathrm{TiO}_{2}$ particles and TAHL aqueous solution are mixed and sonicated, and the PAA is dropwise added to the $\mathrm{TiO}_{2}$ mixture and further mixed and sonicated until becoming homogeneous. The latter, is dropwise added to the oil phase Ag-rich solution and complete homogenization is allowed at $\sim 70^{\circ} \mathrm{C}$. Once homogeneous, the mixture is cooled down by stopping the heat while magnetically stirring. The air bubbles are then introduced with the aid of an electric wisk-like frother for 6-8 min. The Ag precursor added, is calculated to yield $1.2 \mathrm{wt} \%$ of metallic $\mathrm{Ag}$ from the total $\mathrm{TiO}_{2}$ - $\mathrm{Ag}$ final composite (i.e. excluding organic molecules).

Table 3. Ag-rich oil phase constituents.

\begin{tabular}{|c|c|c|c|c|c|}
\hline Precursor & Function & $\begin{array}{l}\text { Chemical } \\
\text { Formula }\end{array}$ & Chemical Structure & $\begin{array}{c}\text { Molecular } \\
\text { Weight } \\
\text { (g/mol) }\end{array}$ & $\begin{array}{c}\text { Concentration } \\
\text { (wt\%) }\end{array}$ \\
\hline Stearic Acid & Oil phase constituen & $\mathrm{CH}_{3}\left(\mathrm{CH}_{2}\right)_{16} \mathrm{COOH}$ & $\mathrm{CH}_{3}\left(\mathrm{CH}_{2}\right)_{15} \mathrm{CH}_{2}{ }_{\mathrm{OH}}$ & 284.304 & 33.44 \\
\hline Lanolin & $\begin{array}{c}\text { Oil phase constituen } \\
\text { Emulsifier }\end{array}$ & ---- & & ---- & 27.84 \\
\hline $\begin{array}{l}\text { Polyoxoethylene } \\
\text { Sorbitan } \\
\text { Monostearate }\end{array}$ & $\begin{array}{c}\text { Oil phase constituen } \\
\text { Surfactant } \\
\text { Emulsifier }\end{array}$ & $\begin{array}{c}\mathrm{C}_{64} \mathrm{H}_{126} \mathrm{O}_{26} \\
x+y+z+w=20\end{array}$ & & 1311.046 & 33.39 \\
\hline Ethanolamine & \multirow{2}{*}{$\begin{array}{c}\text { Oil phase constituen } \\
\text { Surfactant } \\
\text { Emulsifier }\end{array}$} & $\mathrm{NH}_{2} \mathrm{CH}_{2} \mathrm{CH}_{2} \mathrm{OH}$ & & 61.064 & \multirow[b]{2}{*}{8.34} \\
\hline Triethanolamine & & $\mathrm{C}_{6} \mathrm{H}_{15} \mathrm{NO}_{3}$ & & 149.130 & \\
\hline Ethanol & $\begin{array}{l}\text { Solvent for } \\
\text { Ag-precursor }\end{array}$ & $\mathrm{CH}_{3} \mathrm{CH}_{2} \mathrm{OH}$ & & 46.07 & $1 \mathrm{~mL}$ \\
\hline Ag-acetate & Ag precursor & $\mathrm{CH}_{3} \mathrm{COOAg}$ & $\mathrm{CH}_{3}$ & 166.892 & $\begin{array}{c}1.2 \mathrm{wt} \% \\
\text { (0.3 at\%) } \\
\text { of target } \\
\mathrm{TiO}_{2}-\mathrm{Ag} \\
\text { composite }\end{array}$ \\
\hline
\end{tabular}




\section{Ag-Decorated XG $\mathrm{TiO}_{2}$ Inks (Hydrocolloid-based)}

For the synthesis of the oil-free Ag-decorated foams, xanthan gum is used as viscosity enhancer. To prepare the inks, appropriate amounts of $\mathrm{TiO}_{2}$ nanoparticles are mixed with $\mathrm{TALH}$ and DI water. This mixture is stirred for $15 \mathrm{~min}$, and sonicated in a water/ice bath for $15 \mathrm{~min}$ to ensure thorough dispersion (while occasionally stirring to prevent sedimentation). In parallel a PAA - Xanthan gum (XG) aqueous solution is prepared and added dropwise to the titania mixture while stirring. The resulting mixture is sonicated for $15 \mathrm{~min}$ and set to stir for 15 more minutes before frothing. For the encapsulation of the $\mathrm{Ag}$ ions, Ag-acetate is mixed in ethanol and solubilized by adding ammonium hydroxide aqueous solution. The Ag-acetate: $\mathrm{NH}_{4} \mathrm{OH}$ ratio is (1:9) in mol. This Ag-rich solution is added to the PAA-XG solution and allowed to homogenize before being added to the titania mixture. Aluminum foil is wrapped around all vials containing $\mathrm{Ag}$ to prevent light induced reactions or degradation. The inks are frothed for $\sim 8-20 \mathrm{~min}$ using a wisk-like attachment and a mechanical mixer to incorporate air bubbles. The $\mathrm{Ag}$ content is kept identical, and equivalent to $1.2 \mathrm{wt} \%$ of the final $\mathrm{TiO}_{2}$-Ag composite.

Table 4. Ag-decorated XG $\mathrm{TiO}_{2}$ inks constituents.

\begin{tabular}{|c|c|c|c|c|c|}
\hline Precursor & Function & $\begin{array}{l}\text { Chemical } \\
\text { Formula }\end{array}$ & Chemical Structure & $\begin{array}{c}\text { Molecular } \\
\text { Weight } \\
\text { (g/mol) }\end{array}$ & $\begin{array}{c}\text { Concentration } \\
\text { (mol) }\end{array}$ \\
\hline DI Water & Solvent & $\mathrm{H}_{2} \mathrm{O}$ & & 18.015 & 128.4 \\
\hline $\begin{array}{l}\text { Titanium } \\
\text { bis(ammonium } \\
\text { lactato) dihydroxide } \\
\text { - TALH }\end{array}$ & $\mid \begin{array}{c}\text { Ti-organic } \\
\text { (precursor for } \mathrm{TiO}_{2} \\
\text { bridging structures) }\end{array}$ & $\begin{array}{c}{\left[\mathrm{CH}_{3} \mathrm{CH}(\mathrm{O}-\right.} \\
\left.\mathrm{CO}_{2} \mathrm{NH}_{4}\right]_{2} \mathrm{Ti}(\mathrm{OH})_{2}\end{array}$ & $2 \mathrm{NH}_{4}^{+}$ & 294.08 & 1 \\
\hline $\begin{array}{l}\text { Titanium Dioxide } \\
\text { Nanoparticles ( } 20 \\
\text { nm diameter) - } \\
\text { Aeroxide }{ }^{\circledR}\end{array}$ & $\begin{array}{c}\text { Primary particles } \\
\text { (target material } \\
\text { composition) }\end{array}$ & $\mathrm{TiO}_{2}$ & & 79.865 & 12 \\
\hline $\begin{array}{l}\text { Polyacrylic Acid - } \\
\text { PAA }\end{array}$ & $\begin{array}{c}\text { Adhesion promoting } \\
\text { Nozzle-clogging } \\
\text { preventing }\end{array}$ & $\left(\mathrm{C}_{3} \mathrm{H}_{4} \mathrm{O}_{2}\right)_{\mathrm{n}}$ & & 72.033 & 1 \\
\hline $\begin{array}{l}\text { Xanthan Gum - } \\
\text { XG }\end{array}$ & $\begin{array}{l}\text { Rheology } \\
\text { enhancer }\end{array}$ & $\left(\mathrm{C}_{35} \mathrm{H}_{49} \mathrm{O}_{29}\right)_{n}$ & & 933.398 & $7.72 \times 10^{-5}$ \\
\hline Ethanol & $\begin{array}{l}\text { Solvent for } \\
\text { Ag precursor }\end{array}$ & $\mathrm{CH}_{3} \mathrm{CH}_{2} \mathrm{OH}$ & & 46.07 & $1.71 \times 10^{-2}$ \\
\hline $\begin{array}{l}\text { Ammonium } \\
\text { Hydroxide }\end{array}$ & $\begin{array}{l}\text { Solubilizing agent } \\
\text { for Ag precursor }\end{array}$ & $\mathrm{NH}_{4} \mathrm{OH}$ & 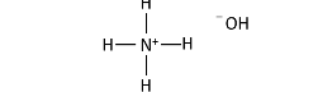 & 35.046 & $3.16 \times 10^{-3}$ \\
\hline Ag-acetate & $\mathrm{Ag}$ precursor & $\mathrm{CH}_{3} \mathrm{COOAg}$ & & 166.892 & $\begin{array}{c}3.52 \times 10^{-4} \\
(1.2 \mathrm{wt} \% \text { of } \\
\text { target } \mathrm{TiO}_{2}-\mathrm{Ag} \\
\text { composite) }\end{array}$ \\
\hline
\end{tabular}




\subsection{Inks Characterization}

The viscosity of the formulated inks was measured with a Brookfield DV-II+ Pro rotational viscometer at various shear rates ranging from 0 to $1000 \mathrm{~s}^{-1}$. Contact angle measurements of the ink and of DI water (as a control fluid) were obtained by image analysis of side-view pictures of $2 \mu \mathrm{L}$ droplets on various substrates (glass, polyethylene naphthalate (PEN), ITO coated polyethylene terephthalate (ITO/PET), and ITO/glass). Thermogravimetric analysis (TGA) of the inks was performed with a Pyris 1 TGA PerkinElmer thermogravimetric analyzer from room temperature to $900^{\circ} \mathrm{C}$ and a heating rate of $5^{\circ} \mathrm{C} / \mathrm{min}$ or $10^{\circ} \mathrm{C} / \mathrm{min}$. For the foams, TGA was performed using a TA instruments analyzer from room temperature to $600^{\circ} \mathrm{C}$ and a heating rate of $10^{\circ} \mathrm{C} / \mathrm{min}$. Particle size was determined by dynamic light scattering (DLS) using a Zetasizer Nano - ZS DSC machine; as well as through electron microscopy observations.

\subsection{Inks Deposition}

Doctor blading (DB) of the films was performed using Scotch tape 3M 600 (58.4 $\mu \mathrm{m}$ thickness) as spacer, on cleaned glass slides, ITO/glass, and/or on ITO/PET OC300/ST504/7mil Solutia ${ }^{\text {TM }}$ substrates. CDW of films and patterns was performed using a commercially available Nordson JR2300N robotic arm, or a labbuilt robotic deposition system with pressure and movement controlling systems in $x-y-z$ directions, equipped with a Performus $V$ pneumatic pressure ink dispenser system. The directly written samples were prepared on various substrates (glass, PEN, ITO/glass, ITO/PET, and flexible Willow $®$ glass CORNING). The direct writing parameters (writing speed and extrusion pressure) were varied to investigate the printing

ranges of the formulated inks keeping a distance to substrate of around $1 / 3$ to $2 / 3 \times$ nozzle diameter for the $\mathrm{Ag}$ and titania inks. The distance was kept to 1 nozzle diameter for the $\mathrm{ZnO}$ and the $\mathrm{TiO}_{2}$ foams. Appropriate values for this distance are highly dependent on the ink viscosity. Stainless steel and polymeric nozzles with inner diameters of 100, 150, 200 and $840 \mu \mathrm{m}$, were used for the ink/foam depositions depending on their rheological and chemistry properties.

UV treatments were performed on the deposited films with a SpectroLINKER ${ }^{\mathrm{TM}}$ XL-1500 Spectroline ${ }^{\circledR}$ UV crosslinker machine with G15T8 bulbs of 15 watt $-254 \mathrm{~nm}$ radiation wavelength. High-temperature annealing was performed in a box furnace KSL 1100X, MIT Corporation, or a muffle furnace equipped with a SMART-3 fuzzy logic temperature controller Temp,Inc. $($; ; and low-temperature annealing in a Lindberg Blue M3057 oven; all annealing processes were held for $30 \mathrm{~min}$ with heating and cooling rates of $5{ }^{\circ} \mathrm{C} / \mathrm{min}$, unless otherwise indicated. For the titania foams, the cooling rate was $1^{\circ} \mathrm{C} / \mathrm{min}$. For the $\mathrm{ZnO}$ structures, drying of the structures was induced by placing the samples on a hotplate at $200{ }^{\circ} \mathrm{C}$ for $10 \mathrm{~min}$. Sintering of these samples was performed at $250,450,500$ and $525^{\circ} \mathrm{C}$, respectively.

Spanning features of $\mathrm{TiO}_{2}$ foams were fabricated on regular and hydrophobized glass slides, using a variation of the method developed by Banerjee et al.[179] Briefly, tetraethoxysilane (TEOS), and perfluoropolyether-alkoxysilane (PFPE) were mixed until homogeneous using a mole ratio of 1:0.005 TEOS:PFPE. In parallel, a $\mathrm{HCl}$ aqueous solution (1:0.025 water: $\mathrm{HCl}$ mole ratio) was added dropwise to the TEOS-PFPE mixture while stirring. The solution was left under stirring for $24 \mathrm{~h}$, after which ethanol was added to match a mole ratio of 1:3.75 water:ethanol. The substrates were dip-coated using a KSV Instruments dip-coater with a withdrawal speed of $50 \mathrm{~mm} / \mathrm{min}$ and allowed to dry for approximately 30 min before curing at $200{ }^{\circ} \mathrm{C}$ for $4 \mathrm{~h}$; the heating and cooling rates are kept to $5{ }^{\circ} \mathrm{C} / \mathrm{min}$. 


\subsection{Films/Patterns Characterization}

Optical images of the printed patterns were taken with a Dino Edge-Digital programmable optical microscope, and a Leica optical microscope (magnifications of 10, 20, 50X) equipped with a Guppy Allied Vision Technology frame grabber. Scanning electron microscope (SEM) images were obtained with a Hitachi S-4700 SEM; SEM and energy dispersive x-ray spectroscopy (EDS) data were taken with a JEOL JSM-7600F SEM. No sample preparation was necessary for imaging of the Ag-patterns or the titania films/patterns on conductive substrates. However, $(\sim 6-8 \mathrm{~nm}) \mathrm{Au} / \mathrm{Pd}$ coatings were deposited for imaging the titania foams prepared on glass. The Ag-decorated $\mathrm{TiO}_{2}$ samples were prepared on ITO glass substrates to avoid further coatings for SEM imaging. Particle size analysis of the Ag surfaces was conducted from image analysis of the SEM pictures with ImageJ NIH software. X-ray diffraction (XRD) patterns were obtained with a Bruker D8 Discovery XRD machine in 1D mode and $40 \mathrm{kV}-40 \mathrm{~mA}$ configuration, and a PANalytical X'Pert Pro X-ray diffractometer with power settings of $45 \mathrm{kV}$ and $40 \mathrm{~mA}$. The data were analyzed with the aid of the X-Pert Highscore Plus PANalytical software.

Electrical resistivity measurements of the Ag-patterns were taken by Hall Effect with an Ecopia HMS-3000 measurement system with a magnetic field of $1.02 \mathrm{~T}$. Light transmittance measurements of the Agpatterns were taken between 500 - $900 \mathrm{~nm}$ wavelengths with a JAZ UV-Vis Spectrometer OceanOptics. Mechanical tensile testing was performed using an ADMET MTESTQuattro mechanical testing unit under monotonic, and cyclic-load testing conditions; for the cyclic testing, a tensile sawthooth profile up to $2 \%$ strain with a servo control displacement rate of $2 \mathrm{~mm} / \mathrm{min}$ was applied. Electrical resistance variation was monitored in-situ using a digital multimeter Agilent 34970A along with a Dino Edge-Digital optical microscope to monitor potential failure mechanisms on the sample surface.

Mechanical flexibility assessment of the $\mathrm{ZnO}$ structures was performed with an ADMET MTESTQuattro mechanical testing unit, using a servo control displacement rate of $2 \mathrm{~mm} / \mathrm{min}$ up to $58.8 \mathrm{~mm}$ bending radius.

For the titania samples, Raman spectra were obtained with a Reinshaw INVIA Raman spectrometer with a $532 \mathrm{~nm}$ wavelength excitation source at $5 \%$ power and $50 \mathrm{X}$ magnification. The acquired spectra were analyzed with the WiRE 3.4 Renishaw software. X-ray photoelectron spectroscopy (XPS) was conducted with a PHI 5000 VERSAPROBE 5700 XPS/UPS machine with $284.8 \mathrm{eV}$ C1s (C-C binding energy) internal calibration standard. A monochromated $25 \mathrm{~W}, 15 \mathrm{kV}$ Al $\mathrm{K}_{\alpha} \mathrm{x}$-ray source (photon energy of $1486.6 \mathrm{eV}$ ) and a hemispherical analyzer were used, at $\sim 5 \times 10^{-10}$ Torr pressure in the main chamber; the acquired spectra were analyzed using the MultiPak v9 software. Transmission electron microscope (TEM) images and selected area electron-diffraction (SAED) patterns were acquired using a JEOL JEM-2100 TEM machine at $200 \mathrm{kV}$ acceleration voltage equipped with a Gatan Erlangshen ES500W digital camera and a Gatan Orius SC600 high-resolution digital camera. Thickness measurements for the directly written samples were performed with a Bruker Dektak XT Profilometer, with a $2 \mu \mathrm{m}$ stainless steel tip. Thickness measurements for the directly written foam films and 3D structures before and after sintering, were obtained though image analysis of optical micrographs using ImageJ software $(\mathrm{NIH})$. Roughness measurements of the samples were performed by profilometry and atomic-force microscopy (AFM). The roughness measurements from profilometry were obtained with the aid of the Vision64 profilometer controller software. AFM images were obtained with an Agilent 5500 SPM atomic force microscope using tapping 
mode. The AFM tip has a radius of $\sim 2-5 \mathrm{~nm}$ and a resonant frequency of $\sim 300 \mathrm{kHz}$, the tip is made of Si with a $100 \mathrm{~nm}$ Al coating on the back side. AFM image analysis was conducted using Gwyddion 2.45 Software.

Microporosity analysis (by monitoring nitrogen gas absorption) of the $\mathrm{TiO}_{2}$ foams, was performed with an ASAP2020 accelerated surface area and porosity system Micromeritics®; degassing of samples for microporosity measurements was performed using a Micromeritics vac-prep system for 24 hours (no heat) and $3 \mathrm{~h}$ at $110^{\circ} \mathrm{C}$.

Heterogeneous photocatalysis degradation of a $10 \mu \mathrm{M}$ methylene blue (MB) aqueous solution was performed by placing samples of $2 \mathrm{~cm} \mathrm{X} 1 \mathrm{~cm}$ active area on ITO/PET substrates, from TALH:TiO $\mathrm{T}_{2}(1: 6)$ and $(1: 12)$ inks in identical beakers, each containing $20 \mathrm{ml}$ of solution. A control solution was also placed in identical conditions and was labeled as Blank. Once immersed in the solution, the samples were left to stabilize for 30 minutes in the dark to allow for dye adsorption on the $\mathrm{TiO}_{2}$. The UV irradiation, in $15 \mathrm{~min}$ steps, was performed in the SpectroLINKER ${ }^{T M}$ XL-1500 Spectroline ${ }^{\circledR}$ UV crosslinker machine at $254 \mathrm{~nm}$ radiation wavelength. The samples were placed at $9.5 \mathrm{~cm}$ distance from the bulbs, which deliver an average intensity of $\sim 6000 \mu \mathrm{W} / \mathrm{cm}^{2}$. Light absorbance of MB solutions was measured through UV-Visible spectroscopy with a Lambda35 ${ }^{\mathrm{TM}}$ UV-Vis spectrometer PerkinElmer, or a JAZ UV-Vis spectrometer OceanOptics. The spectra were taken from $196 \mathrm{~nm}$ to $1100 \mathrm{~nm}$ wavelength in polystyrene disposable cuvettes

UV-Vis measurements of $\mathrm{Ag}^{-\mathrm{TiO}_{2}}$ composite films were taken using a Shimadzu UV-2600 spectrophotometer with an integrating sphere, and equipped with a D2 deuterium lamp and a WI halogen lamp for the ultra-violet and visible/near-infrared spectrum ranges, respectively.

Films and devices IV-characterization was executed with an Agilent 4155C semiconductor parameter analyzer station. Variation of the light conditions was achieved with the aid of an Acton SP-150 monochromator, equipped with a TS-428 tungsten/halogen lamp, interfaced with the SpectraSense software - Princeton Instruments. The ambient (dark) and specific wavelength light intensities were measured with a PM100D compact power and energy meter console - Thorlabs. 


\section{Chapter 3: Ag System}

The increasing demand for conducting, yet optically transparent/translucent materials for opto-electronic applications $[94,113]$, makes metallic thin-films and patterns on flexible substrates alternative candidates to replace transparent conducting oxides (TCOs). This is due to their broad utilization as contact and interconnect materials for device integration in modules and large-area panels[71,73,112]. Therefore, investigations on the synthesis, printing, processing and performance of metal nanoparticle conductive inks, is of paramount importance for the additive manufacturing of such components.

The investigated Ag ink system, consists of Ag nanoparticles stabilized with PAA as capping layer, and dispersed in a cellulose aqueous solution. The use of different reducing agents and the Ag-particle synthesis conditions are investigated to control the particle size and product yield. Also, the rheological and printing parameters for robotic deposition of the synthesized inks are explored. Finally, characterization of the microstructural, optical, electrical and mechanical properties, is performed to establish relationships with the materials printing and processing conditions.
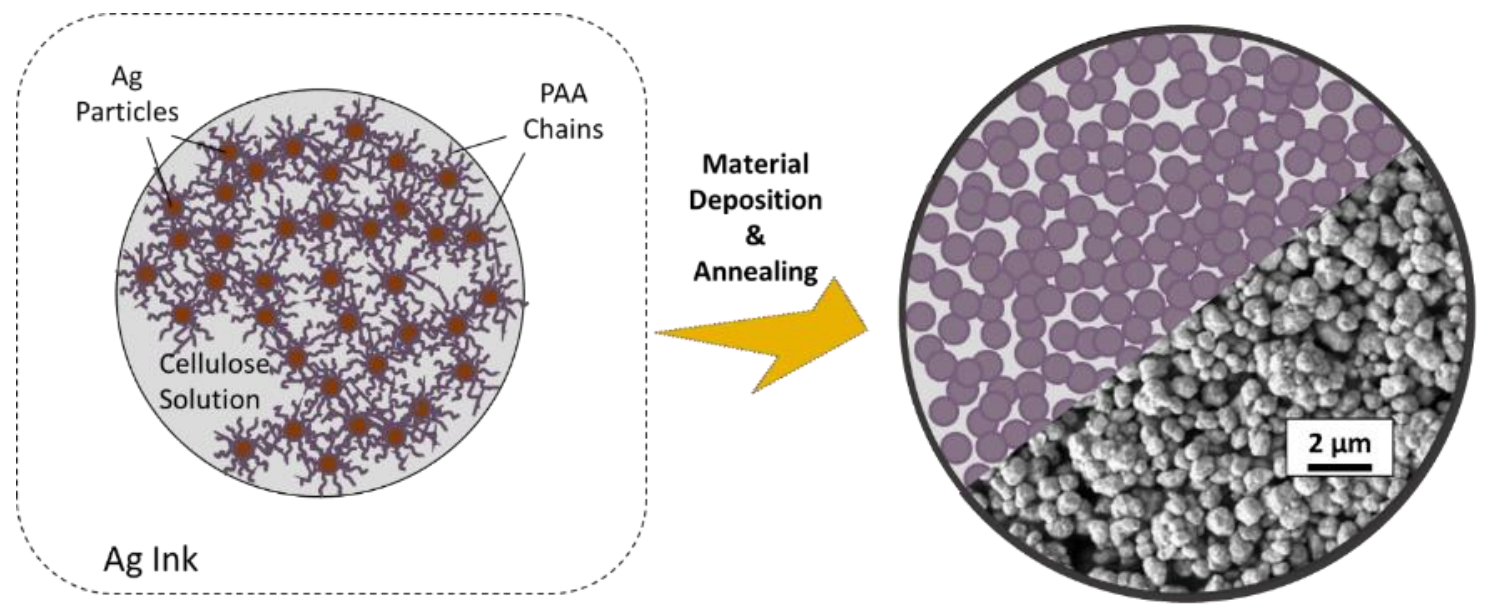

Figure 8. Ag particle ink system.

\subsection{Ag Particle Nucleation Process: Influence of $\mathrm{pH}$, Viscosity and Temperature}

The nucleation of $\mathrm{Ag}$ nanoparticles from ionic solutions in the presence of a reducing agent is a rather widespread synthesis route. Basically, a Ag-precursor is solubilized and mixed with a polymeric capping agent, which prevents the uncontrolled aggregation and growth of the particles[6,19]. The reducing agent on the other hand, triggers the particle nucleation and growth[19].

The initial synthesis route adopted from Ahn et al.[121], used DEA as reducing agent at room temperature $\sim 25^{\circ} \mathrm{C}$, yielding approximately $10 \%$ of the targeted $\mathrm{Ag}$ yield $(100 \%)$. The main challenge in the process is the great kinetic and electrostatic stability of the obtained Ag nanoparticles ( 5 to $10 \mathrm{~nm}$ as measured using DLS) in the precursor solution impairing their extraction, see Figure 9. The substitution of DEA for ethanolamine, also known as mono-ethanolamine (MEA) exhibiting lower boiling temperature than diethanolamine[180], signified the potential elimination of the particle extraction stage from the precursor solution (at early stages of the ink design, for this investigation) enabling low-temperature fabrication and maximizing the $\mathrm{Ag}$ particle yield. 


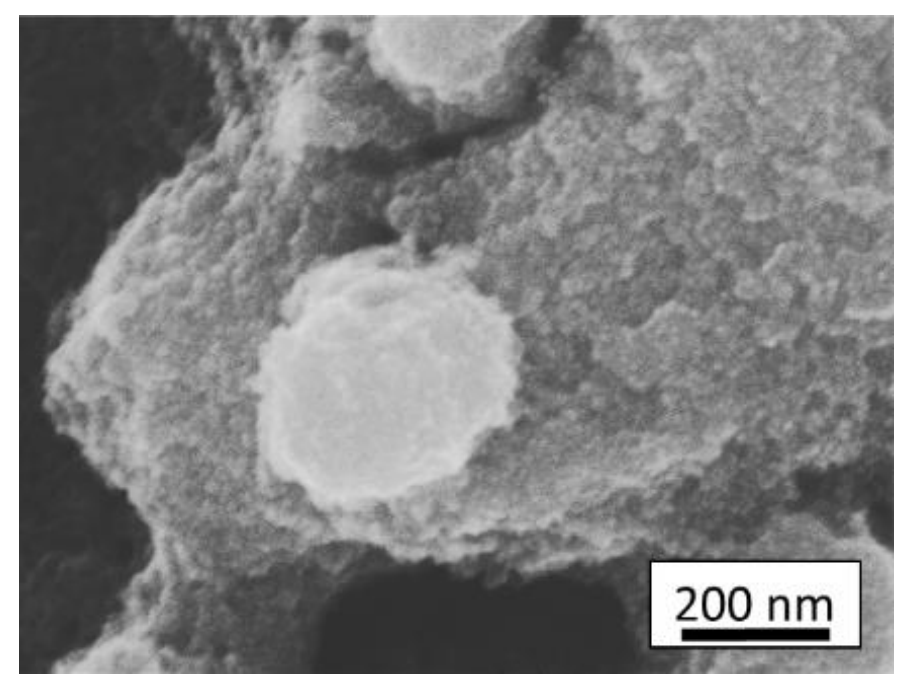

Figure 9. SEM image of the as prepared silver nanoparticles from DEA solutions.

The exchange of DEA for MEA in the synthesis route, resulted in very slow nucleation of the particles (the process which customary took $\sim 24$ hours, resulted in nucleation times of $\sim 1$ week to yield a solution with similar coloration), which is mainly attributed to the higher $\mathrm{pH}$ of MEA when compared to DEA. Accordingly, a decrease in the amount of reducing agent (MEA) was implemented, implying a more economical fabrication route, and potentially facilitating the particle extraction by centrifugation due to the lower viscosity exhibited by MEA when compared to DEA (16.2 and $380 \mathrm{CP}$, respectively at $\left.30^{\circ} \mathrm{C}[180]\right)$. The particle nucleation, monitored by $\mathrm{Ag} / \mathrm{PAA}$ solids yield, was found to be approximately equivalent for equivalent $\mathrm{pH}$ conditions of MEA and DEA, yielding $11 \%$ of $\mathrm{Ag} / \mathrm{PAA}$ solids. Increasing the temperature during nucleation (using MEA) resulted in increased particle size to $\sim 280 \mathrm{~nm}$, see Figure 10 . The remaining precursor solution, a Ag-concentrated fluid, was kept undisturbed in the dark to allow for particle sedimentation and further extraction. After 15 days, the sediments from the precursor solution were recovered by centrifugation with a Ag/PAA yield of $\sim 61 \%$ and an average particle size of $\sim 300 \mathrm{~nm}$, suggesting a particle growth of $\sim 7.41 \%$. This particle growth behavior provides information about the critical factors involved in the process, i.e. time and temperature; the latter exhibiting a more pronounced effect.

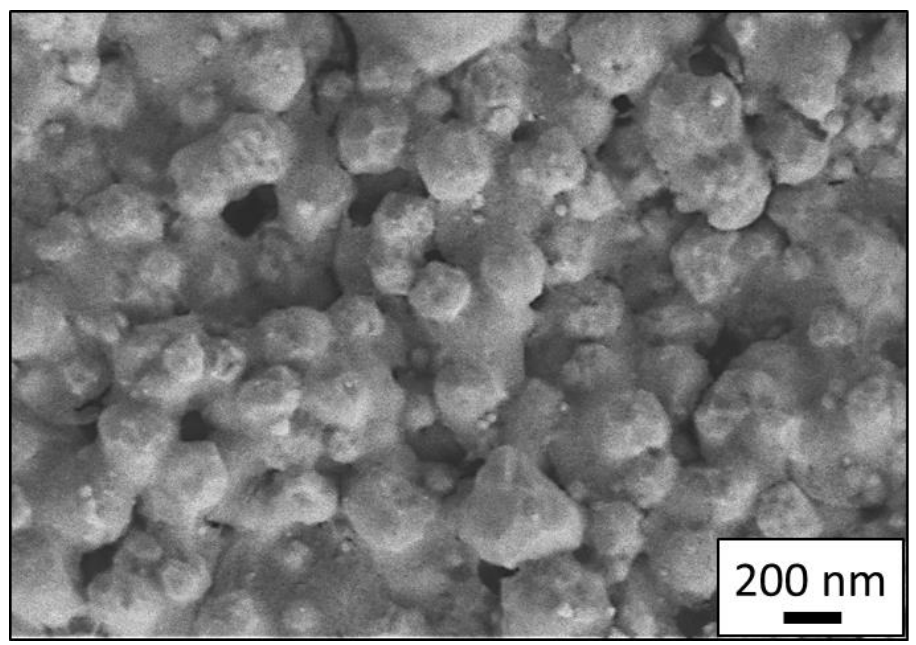

Figure 10. SEM image of the 15 days aged particles. 


\subsection{Ink Properties and Robotic Deposition}

The formulated Ag ink, with an approximate composition of Ag/PAA particles 70 wt $\%, 29.4$ wt $\%$ water and 0.6 wt\% methyl cellulose, exhibits a shear-thinning behavior as indicated by the viscosity measurements (see Figure 11).

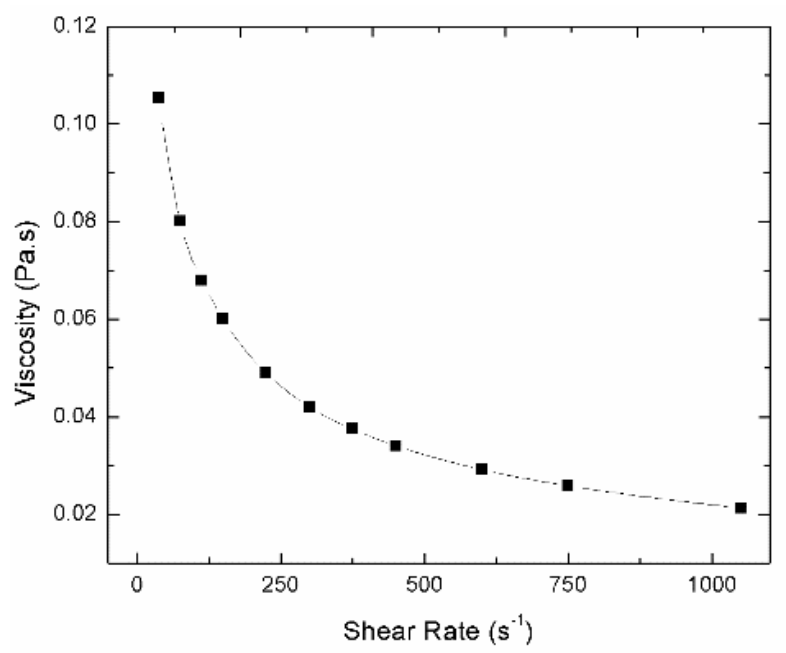

Figure 11. Viscosity as a function of shear rate for the prepared Ag ink.

The concomitant relation between the printing parameters and the ink properties is of great importance when utilizing robotic deposition. Based on the viscosity ranges of the prepared Ag ink, and using a nozzle to substrate clearance of $\sim 1 / 3$ of the inner nozzle diameter, printing speeds between 3 and $10 \mathrm{~mm} / \mathrm{s}$ and ink extrusion pressures of 69 to $140 \mathrm{kPa}$, led to patterns with line width in the $\mu \mathrm{m}$ range. Speed/pressure combinations, such as $7 \mathrm{~mm} / \mathrm{s}$ and $138 \mathrm{kPa}$, result in well-defined patterns. The ink spreading for this working combination is $\sim 2$ times the initial ink-filament diameter (the same as the nozzle inner diameter). Controlling of the spreading can be realized by using lower pressure, or by increasing the writing speed as shown in Figure 12. Depending on the application, anchoring of the inks to the substrate as a critical adhesive characteristic, may benefit from relatively good wetting/spreading of the inks.

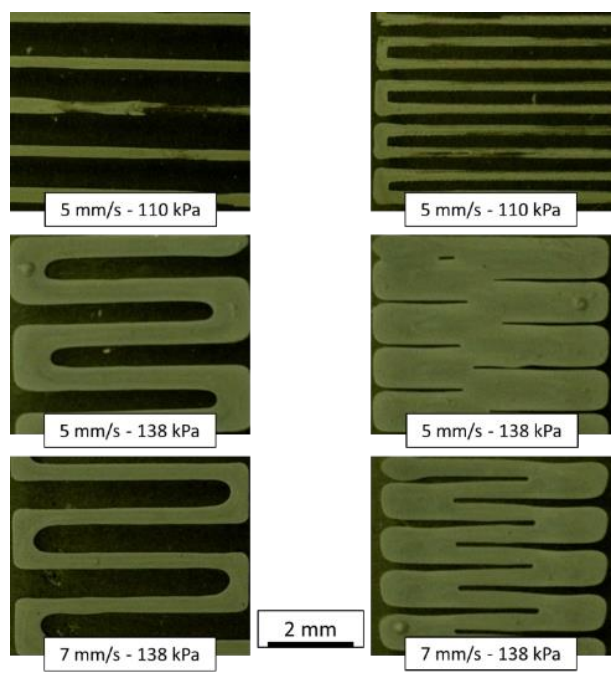

Figure 12. Ag-printed patterns on PEN. Effect of printing speed and pressure. 
Consequently, the pattern feature sizes influence other properties such amount of transmitted light through the sample pattern, see Figure 13. An inverse relation between the area covered by the printed Ag-patterns and the transmitted light (measured from UV-Vis spectrometry) is observed for all the fabricated designs. Higher light transmission is measured for patterns with smaller Ag-covered area (parallel lines with average width of $300 \mu \mathrm{m}$ and variable center-to-center spacing of $500 \mu \mathrm{m}$ and 1000 $\mu \mathrm{m})$. Conversely, for the grid patterns, and wider feature sized patterns, the area covered by $\mathrm{Ag}$ is larger, and their optical transmittance is compromised. The UV-Vis spectra show oscillating lines which are characteristic of Fabry-Pérot fringes resulting from the different materials refractive indexes (Ag and PEN) and the interference of partially transmitted and reflected light at their interfaces (Ag surface/air, Ag/PEN and PEN/air) [181,182].
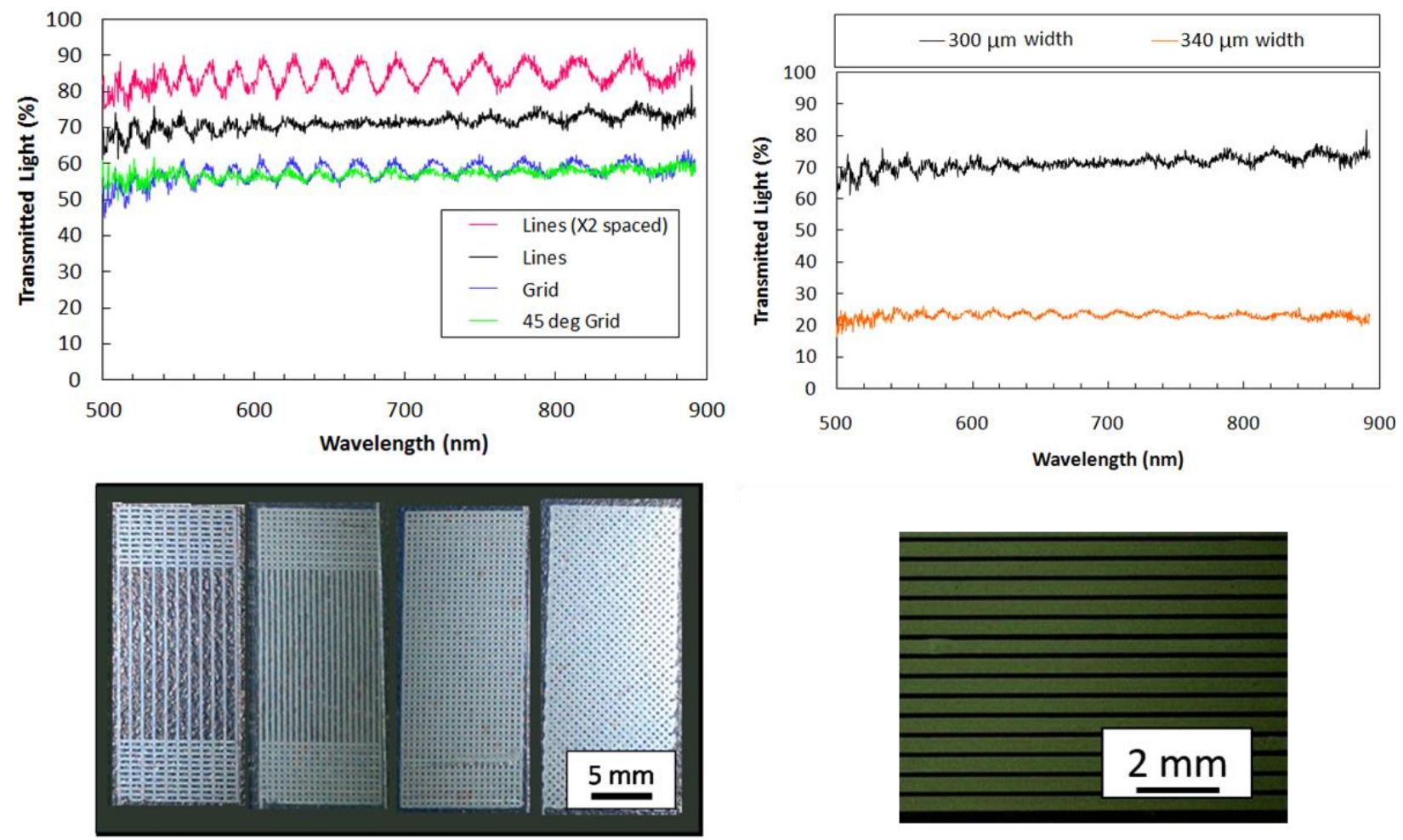

Average line width of $300 \mu \mathrm{m}$

\section{Average line width of $340 \mu \mathrm{m}$}

Figure 13. Transmitted light (UV-Vis measurements) and corresponding patterns with line width of $300 \mu \mathrm{m}$.

\subsection{Patterns Microstructure and Electromechanical Properties}

Lower electrical resistivity values of the printed patterns are obtained as the annealing temperatures are increased (see Figure 14). The measured values are comparable to those obtained for similar Ag-particle based systems[122] (involving higher temperature post-treatments); and to the highest reported value $\left(1.46 \times 10^{-4} \Omega \mathrm{cm}\right)$ of electrical resistivity for annealed ITO on glass[21]. The resistivity values for bulk silver, in the order of $10^{-6} \Omega \mathrm{cm}$, indicate the potential for better performance from this material system. In particular, further understanding of the inter-particle surface binding phenomena and the interactions with the polymeric capping and stabilizing compound, may lead to better electrical conducting properties; 
particularly it has been demonstrated that the stabilization effects of PAA may be tuned after printing by applying ionic salt solutions that are able to trigger the coalescence of $\mathrm{Ag}$ nanoparticles[6]. Additional means to mitigate the counterproductive effects of remnant organic species include the use of alternative hydrocolloids such as xanthan gum or guar gum replacing cellulose, which may be beneficial by reducing the amount of organics in the ink formulation.

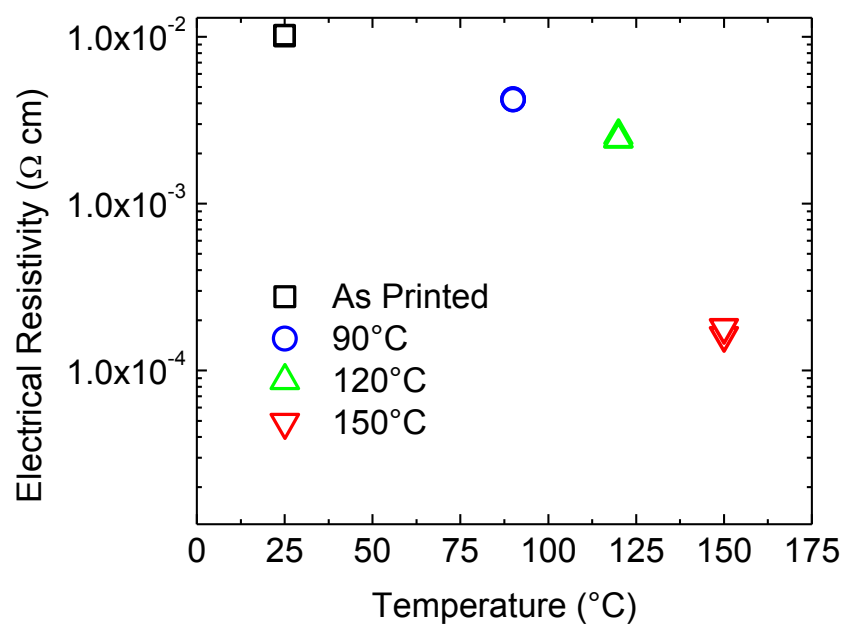

Figure 14. Electrical resistivity of the Ag patterns as measured by Hall Effect.

SEM observations of the annealed Ag patterns show surface morphologies with spherical particles of $\sim 300$ $\mathrm{nm}$, as calculated from image analysis. A slight increase in particle size is observed as annealing temperature increases; in agreement with the electrical resistivity trend, decreasing for increasing annealing temperature.
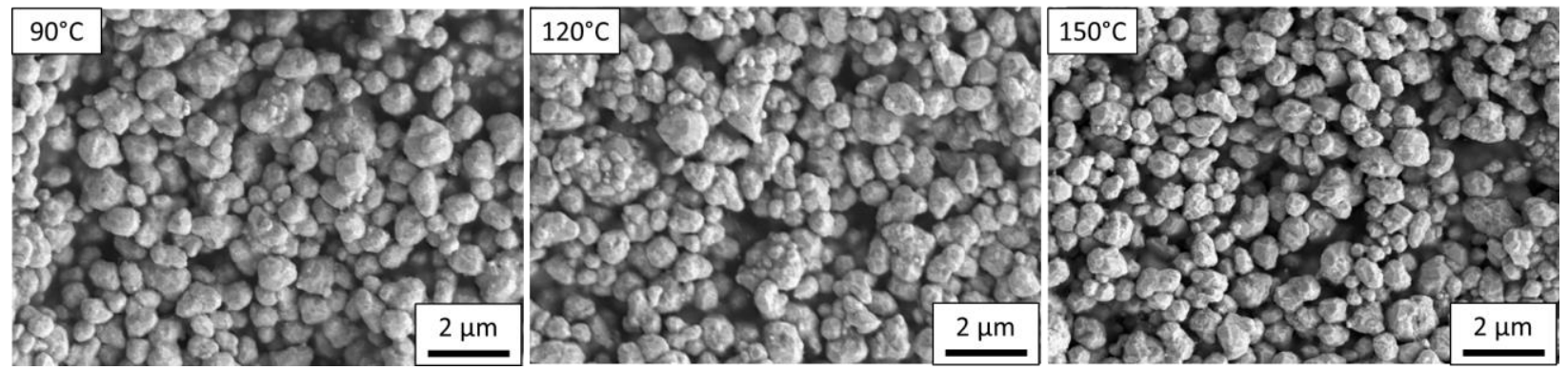

Figure 15. SEM images of the patterns surface after different heat treatments.

The obtained XRD patterns, confirm the crystalline nature of the CDW Ag features to be metallic-Ag in accordance with PDF 00-004-0783; and the grain size increasing trend with increasing annealing temperature (calculated using Scherrer's formula[183]). The broad peaks around $2 \theta$ values of $49^{\circ}$ and $55.5^{\circ}$ correspond to the PEN substrate. Additionally, slight shifting of the (111) peak position (at $38.2^{\circ}$ ) is noted which may suggest the presence of residual tensile stress in the directly written Ag planar structures (see Figure 16 (b). 

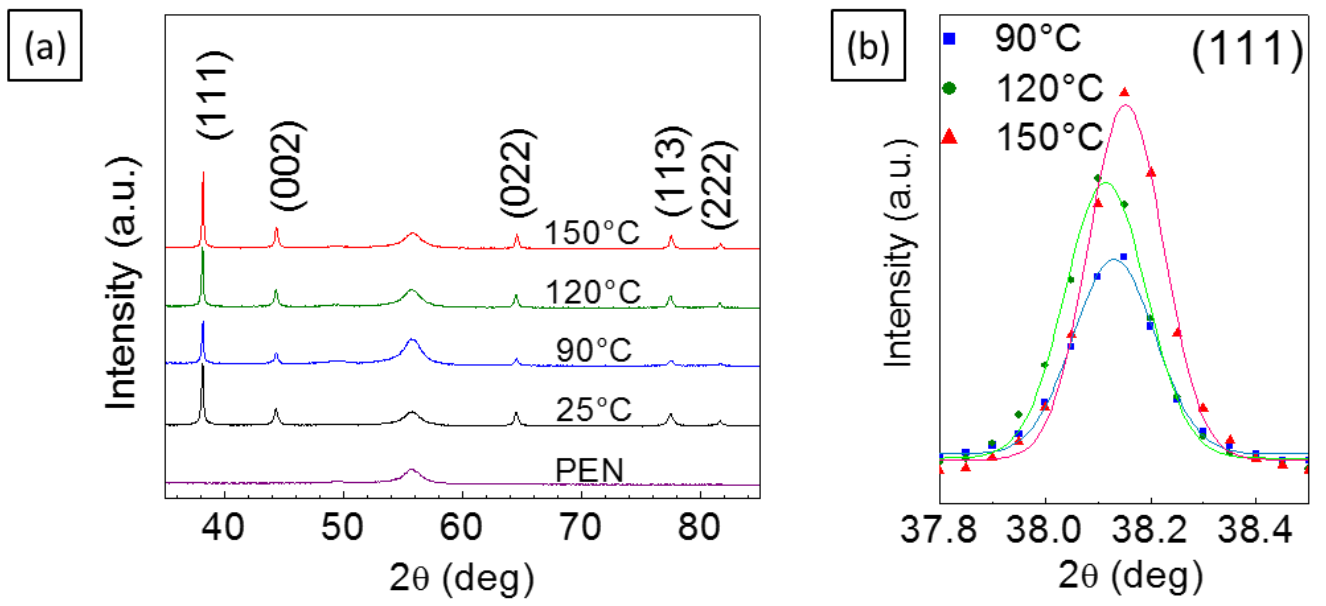

Figure 16. (a) XRD patterns of the CDW Ag arrays after different annealing treatments and (b) Gaussian fit of the (111) reflection.

Table 5. Surface particle size, and Ag grain size dependence on annealing temperature.

\begin{tabular}{ccc}
\hline Temperature $\left({ }^{\circ} \mathbf{C}\right)$ & ${ }^{*}$ Average Particle Size $(\mathbf{n m})$ & ${ }^{* *}$ Grain Size $(\mathbf{n m})$ \\
\hline $\mathbf{2 5}$ & $299.0 \pm 188.6$ & 34.853 \\
$\mathbf{9 0}$ & $288.2 \pm 178.0$ & 46.934 \\
$\mathbf{1 2 0}$ & $309.6 \pm 160.9$ & 47.313 \\
$\mathbf{1 5 0}$ & $341.0 \pm 182.2$ & 50.989 \\
\hline
\end{tabular}

* From SEM image analysis

** From XRD data using Scherrer's approximation

The monotonic uniaxial tensile characterization of Ag micro-patterned lines, suggests that the Ag film does not fail for up to $10 \%$ applied strain; while the acrylic-based layer ( 2-4 nm, coated on the PEN surface to promote adhesion [184]) fails by forming cracks perpendicular to the applied stress direction (observed using in-situ optical microscopy, see Figure 17).

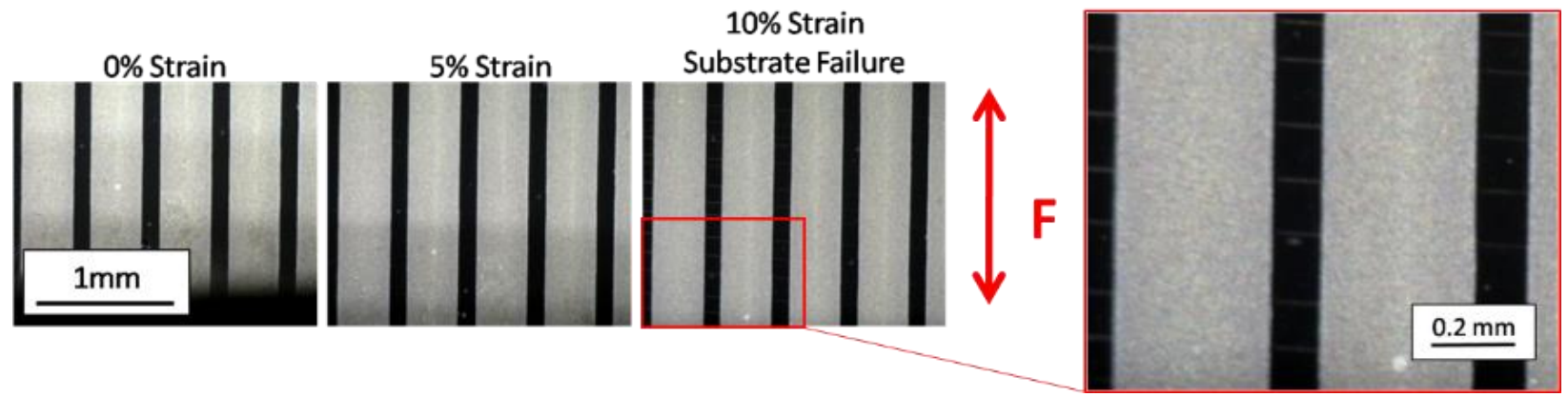

Figure 17. Optical images of the printed line pattern under monotonic tensile stress.

Investigation of the printed $\mathrm{Ag}$ patterns performance under cyclic tensile loading conditions using $2 \%$ strain (the failure threshold for most TCO's on polymeric substrates $[8,9,185]$ ), show that the $\mathrm{Ag}$ patterns remain intact at significantly higher strains than those TCOs can sustain [186]. An arbitrary $20 \%$ increase in electrical resistance is taken as the onset for potential device failure. Additionally, an electrical recovery 
behavior upon release of the tensile load is observed, this behavior may be attributed to the organicinorganic nature of the ink and the low-temperature annealing treatment restricting the $\mathrm{Ag}$ particle coalescence and necking [187]. During stress application, the distance between the particles increases along the applied stress direction, resulting in a slight increase of the pattern's electrical resistance due to the fewer conducting paths available; upon stress release, the distance between the particles in the film is restored leading to electrical resistance recovery by re-establishment of the electron-conduction paths. Consequently, these printed structures may be considered for alternative applications such as printed strain sensors, electrically conductive printed structures, etc.

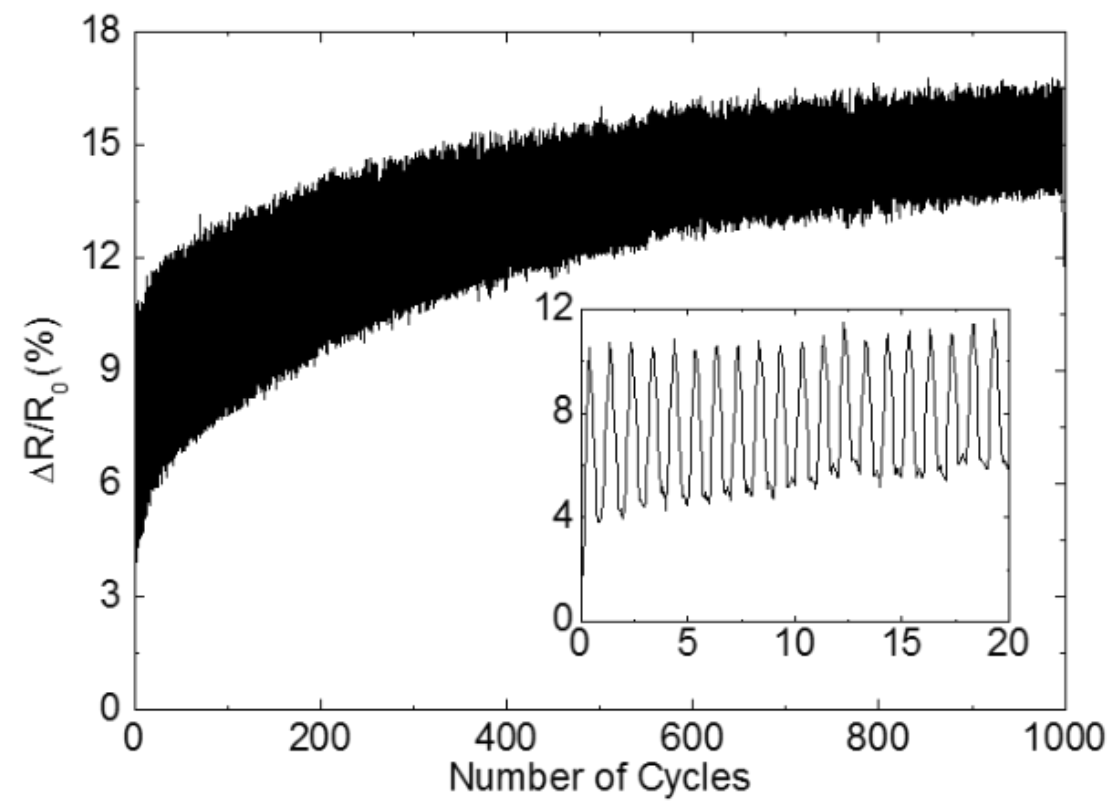

Figure 18. Change in electrical resistance of the printed line pattern as a function of cyclic tensile stress loading.

From the SEM images (se Figure 19), no evidence of structural damage of the Ag film or the PEN substrate is identified. Upon tensile stress application, the sample (substrate and printed Ag pattern) deforms; due ductile nature of metallic $\mathrm{Ag}$ and to its elastic properties, the patterned Ag-film is capable to withstand the load within its elastic region [188]. The cumulative increase in electrical resistance upon cyclic loading (under the failure limit), may be attributed to slight neighboring particle relative displacements where the polymeric material acts as a binding and damping medium. The absence of delamination suggests that the applied stress is transferred from the substrate to the film and not arrested at the film/substrate interface; and moreover, not generating dislocation multiplication within the film. The film's porous microstructure, allows for dislocation annihilation[189], in turn leading to reliable performance under cyclic stress conditions[187]. 

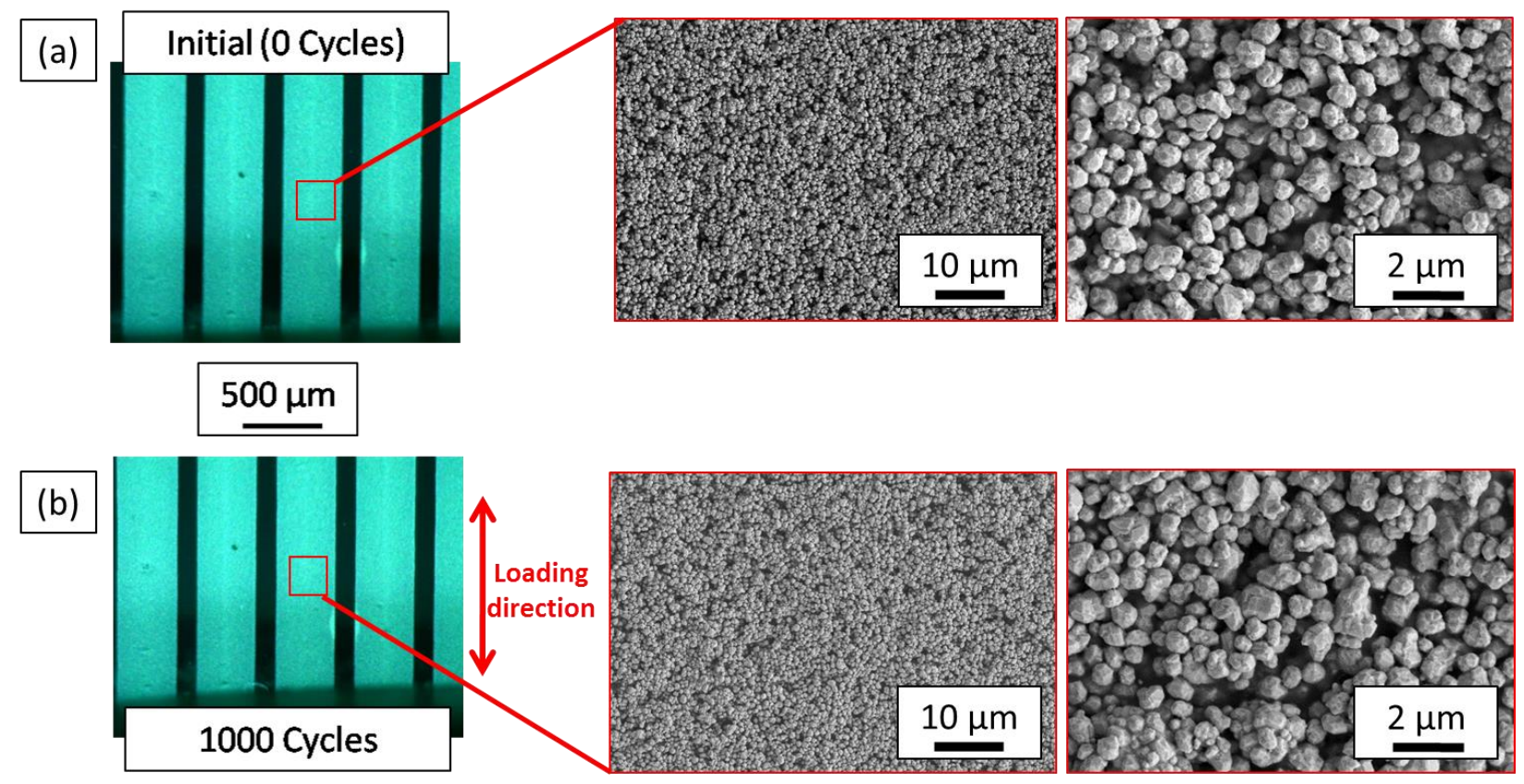

Figure 19. Optical microscope and SEM images of the printed line pattern (a) before and (b) after cyclic tensile stress characterization.

\subsection{Concluding Remarks}

Through the investigation of this ink system, important information about the nucleation/growth - time/ temperature relationships were established, allowing the manipulation of the processing parameters to increase the Ag particle yield with a controlled particle size increment. The studies of the printed conductive patterns on plastics from the formulated ink system, indicate that the electrical resistivity values obtained at lower annealing temperature conditions are comparable to similar Ag printed systems, and are susceptible to improvement, highlighting the potential for controlling the optical transmittance properties by varying the $\mathrm{Ag}$ area coverage and the resolution of the printed features via the CDW printing parameters; for instance, Ag structures with resolution of $\sim 5 \mu \mathrm{m}$ have been reported using solution-based low viscosity inks[94]. The electro-mechanical characterization of the printed samples indicates that the fabricated electrodes (substrate-Ag printed assembly) are relatively robust systems, displaying failure of the substrate before the silver pattern at $\sim 10 \%$ strain (monotonic loading). Uniaxial tensile cyclic testing at $2 \%$ strain, exhibits electrical conductivity recovery behavior upon stress release, highlighting this material system potential for alternative applications such as strain sensors, with a cumulative electrical resistivity increase of $\sim 16.5 \%$ after 1000 cycles which is lower than the threshold for onset of failure of conducting films (arbitrarily set at 20\%). No evidence of structural damage as cracking or delamination of the porous Ag microstructure is observed, resembling relatively reliable structures for cyclic stress conditions, at low strains. This is thought to be related to the microstructural properties of the films that allow for dislocation annihilation at the particle-particle interfaces. Further characterization of the stress threshold values and higher number of stress cycles are proposed. 


\section{Chapter 4: ZnO System}

The Al-doped ZnO (AZO) sol-gel ink system, consisting of Zn-acetate and Al-nitrate dissolved in 2-methoxy ethanol, and using ethanolamine as stabilizer, and PVP as viscosity enhancer; is used to investigate important interactions between the AZO structures' processing and microstructural properties. As highlighted in the work of Abidakun [190], the CDW printing conditions affect the morphology and crystalline structure of the AZO patterns. For this system, the variation of the printing parameters and viscosity properties of the inks resulted in variation of the preferential crystalline orientation of the patterned films. However, further investigation of the texturing mechanism was still necessary, since there was uncertainty about the evolution of the microstructure during sintering.

\subsection{Rheology and Ink Substrate Interactions}

The measured viscosity values for the 15 and 20 wt\% PVP are presented Figure 20 (a), a shear-thinning behavior is observed for all the prepared ink systems, that is more significant as the polymer content is increased. Such shear-thinning profile is observed to deviate form a linear decreasing pattern for the lower range of shear-rates up to $\sim 20 \mathrm{~s}^{-1}$.
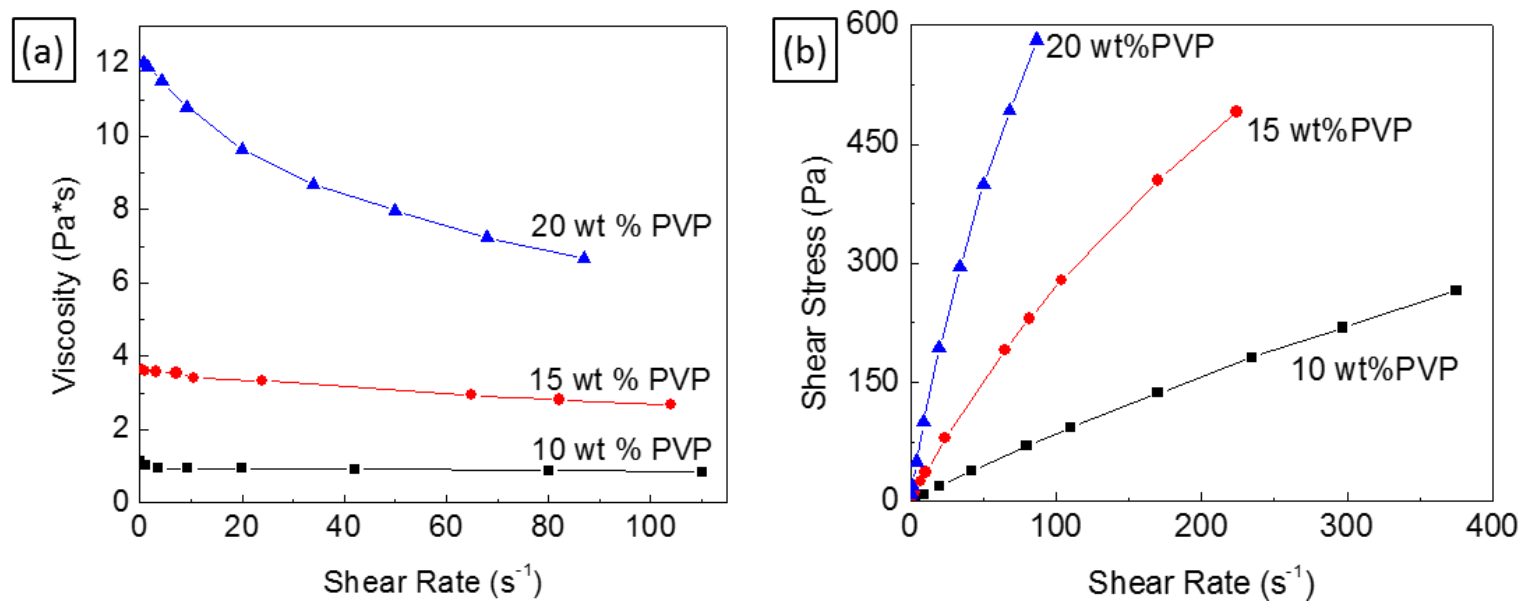

Figure 20. (a) Dynamic viscosity versus shear rate, and (b) shear-stress variation with shear-rate for the AZO inks with different PVP content.

Analysis of the shear-stress variation with shear-rate - Figure 20 (b) - using the Herschel-Bulkley fluids model (see Eq. 1), yields the $\mathrm{k}$ and $\mathrm{n}$ parameters as summarized in Table 6. It is observed that the power law index $\mathrm{n}$ is smaller than the unity, which indicates a shear-thinning behavior. This index is however very close to the unity, so that the inks could approximately be treated as Bingham-plastic fluids [149]. Thus, the inks will exhibit an initial opposition to flow equivalent to the yield stress, and once surpassed will exhibit a shear-stress that increases nearly linearly with the shear rate. The shear-stress dependence on shear-rate is shown in Figure 20, from which only a slight shear-thinning trend can be observed.

Table 6. Yield stress and Herschel-Bulkley parameters for the studied AZO sol-gel inks.

\begin{tabular}{ccccc}
\hline Ink & Yield Stress $\left(\tau_{\mathbf{y}}\right)$ & $\mathbf{k}$ & $\mathbf{n}$ & $\mathbf{R}^{\mathbf{2}}$ (fit) \\
\hline AZO 10 wt\% PVP & 4.7373 & 1.2647 & 0.9897 & 0.9961 \\
AZO 15 wt\% PVP & 17.3980 & 3.7213 & 0.9071 & 0.9942 \\
AZO 20 wt\% PVP & 31.3380 & 9.3951 & 0.9274 & 0.9927 \\
\hline
\end{tabular}


For CDW, the ink/substrate interactions are of paramount importance influencing the adhesion and spreading of the inks onto the substrates. The viscosity [107] and surface energy [191] of the inks are determining factors that affect the geometry and resolution of printed features. The contact angles formed by the inks on cleaned glass substrates provide an indication of their surface energies, and account for the substrate surface wettability by the ink. The higher the contact angle, the lower the wettability of the substrate surface by the ink. Measured contact angles are observed to increase as the PVP content increases from 10 to $20 \mathrm{wt} \%$, with average values of $51.6^{\circ}, 64.2^{\circ}$ and $73.4^{\circ}$ for the inks with 10,15 and 20 wt\% PVP, respectively[131].

The work of adhesion $\left(W_{a}\right)$ for the ink/substrate system (see Eq. 2 ), can be calculated from the contact angle $(\theta)$ measurements[192], and gives values of $69.42,61.50$ and $55.14 \mathrm{~mJ} / \mathrm{m}^{2}$, for the 10,15 and 20 wt\% PVP content inks, respectively. The correspondence between the increasing contact angle and the increasing polymer content, explains the better wetting properties of the lower content PVP formulations. The surface tension $\left(\gamma_{L}\right)$ value used for such estimations is $42.8 \mathrm{~mJ} / \mathrm{m}^{2}$, which is based on the independent solvent and polymer surface tension values[193,194], and considers the rather small variability in the surface tension[195] values for the polymer concentration ranges used in the formulations.

$$
W_{a}=\gamma_{L}(\cos \theta+1)
$$

An additional compositional factor influencing the ink/substrate interactions is the $\mathrm{pK}_{\alpha}$ of the different inks, as it decreases slightly when the amount of PVP is increased (i.e. increasing the polymer to stabilizer (ethanolamine) ratio). Generally, the larger the difference between the ink and the substrate $\mathrm{pK}_{\alpha}$ properties, the better is their anchoring (i.e. adhesion of ink to the substrate). The later observation is in agreement with the contact angle measurements, which exhibit better wetting as PVP concentration is decreased.

\subsection{Texturing Microstructure Through Printing}

Figure 21 shows the effect of different printing parameter combinations on line width, increasing as the volumetric flow-rate increases (i.e. more material is deposited on the substrate). This relationship between the printing parameters, ink viscosity and final AZO dimensions has also significant effects on the resulting microstructure[131,190]; as can be seen from the XRD patterns, Figure 22. Briefly, the larger the printed feature width is, resulting from specific combinations of printing parameters and ink viscosities, the more random is the crystalline orientation of the AZO films.
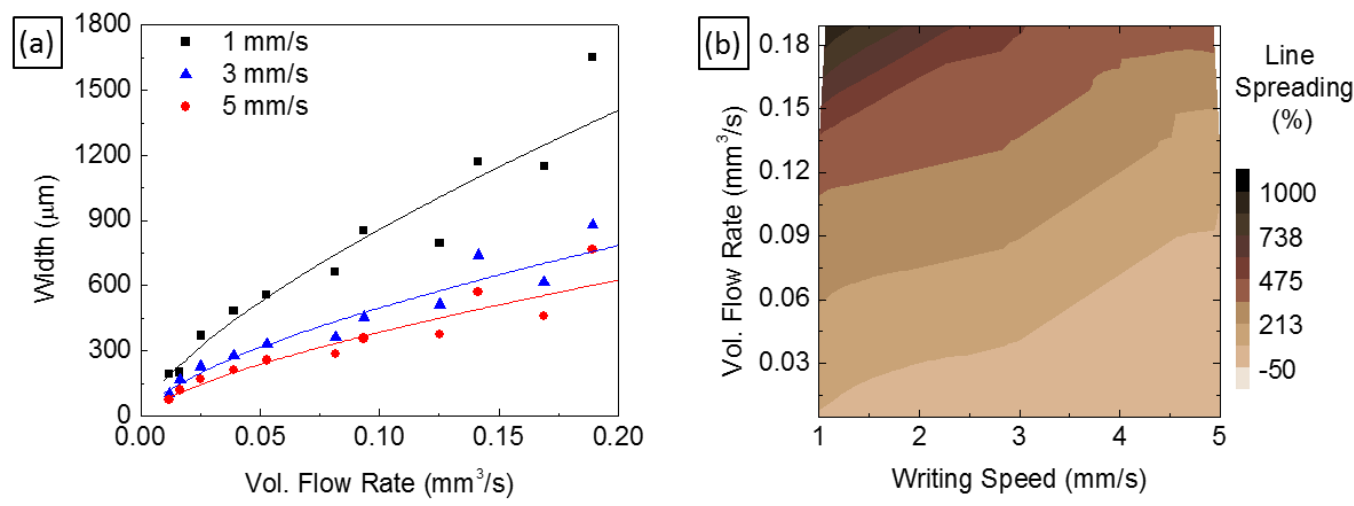

Figure 21.Dependence of printed feature's width (a), and spreading (b) on writing speed and volumetric flow rate. 

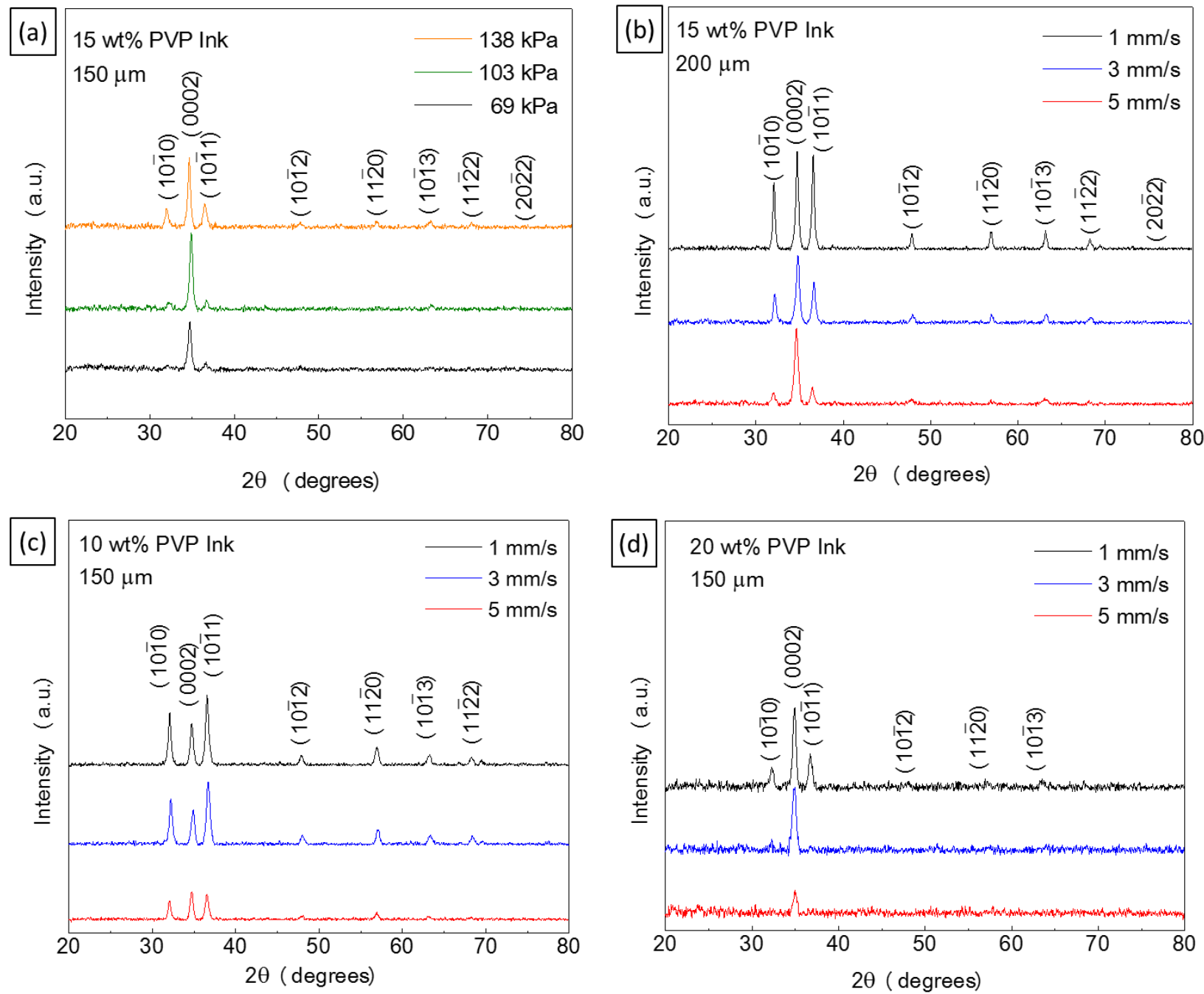

Figure 22. XRD patterns of films printed from ( $a-b) 15$ wt\% PVP inks at (a) $150 \mu \mathrm{m}$ nozzle, $3 \mathrm{~mm} / \mathrm{s}$ speed, and variable pressure; and (b) $200 \mu \mathrm{m}$ nozzle, $103 \mathrm{kPa}$ pressure, and variable speed; and of films printed at various speeds with $150 \mu \mathrm{m}$ nozzles and $103 \mathrm{kPa}$ from (c) 10 wt\% PVP and (d) 20 wt\% PVP inks.

Figure 23 shows the corresponding Lotgering factor (LF), indicating the texturing degree along the 0002 direction, as calculated from the XRD patterns of AZO prints with different widths. JCPDS 36-1451 for undoped polycrystalline powdered $\mathrm{ZnO}$ is taken as reference for the calculation of $p_{0}$. The Lotgering factor accounts for the texturing degree of the crystallized films[196], and is defined for a specific family of planes $\{$ hkil\} as:

$$
\begin{gathered}
L F=\frac{p_{\{h k i l\}}-p_{0}}{1-p_{0}} \\
p_{\{h k i l\}}=\frac{\sum I_{\{\text {hkil }\}}}{\sum I \text { (all refflections) }}
\end{gathered}
$$

Where, $p_{\{h k i l\}}$ is the intensity factor for the family of peaks $\{h k i l\}$ of particular texturing, $p_{0}$ is equivalent to $p_{\{h k i l\}}$ from a randomly oriented powder specimen, and $I$ is the $x$-ray diffraction peak intensity[196]; peak area ratios can also be used to calculate LF values[197]. 


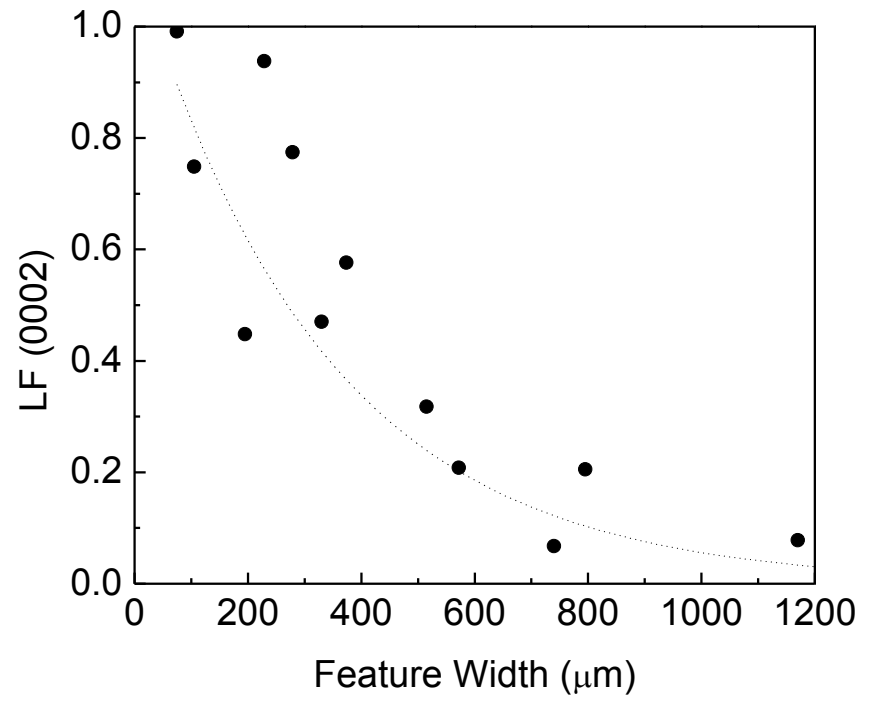

Figure 23. Lotgering factor for (0002) reflections dependence on the width of the directly written AZO structures as indication of their texturing degree.

Thus, it is observed that the texturing of the samples is related to their width, in turn highly dependent on the CDW printing parameters.

The lattice parameters $a$ and $c$, calculated from the XRD patterns of samples with different PVP content, and printed using $3 \mathrm{~mm} / \mathrm{s}$ writing speed and $103 \mathrm{kPa}$ dispensing pressure, using $150 \mu \mathrm{m}$ nozzles; and treated at $500^{\circ} \mathrm{C}$ for 2 hours, are summarized in Table 7. These, show no significant variation between the different PVP content formulations, which demonstrates that the rheological control agent does not induce changes of the crystal unit cell. The calculated lattice parameters are generally smaller than those reported for undoped ZnO (JCPDS 36-1451). Such difference corresponds to 1.25 and $1.43 \%$ for $c$ and $a$ respectively, and may be expected due to the smaller size of the Al atoms when compared to $\mathrm{Zn}$ atoms in this n-type semiconductor[198].

Table 7. lattice parameters for the AZO (Al0.016Zno.984O) structures, from inks with different PVP content in wt\%

\begin{tabular}{cccc}
\hline Sample & $\boldsymbol{c}(\AA)$ & $\boldsymbol{a}(\AA)$ & $c / a$ Ratio \\
\hline AZO 10 wt\% PVP & 5.1375 & 3.2016 & 1.6047 \\
AZO 15 wt\% PVP & 5.1328 & 3.2000 & 1.6055 \\
AZO 20 wt\% PVP & 5.1375 & 3.2000 & 1.6055 \\
ZnO JCPDS 36-1451 & 5.2009 & 3.2471 & 1.6017 \\
\hline
\end{tabular}

\subsection{Morphology Tuning and Texturing Mechanism}

The microstructural evolution of the printed AZO films (from the ink to the crystallized films), is of interest due to the anisotropic properties of $\mathrm{ZnO}[199]$, which could be targeted for specific applications. Thus, from the reported TGA profiles of the AZO inks (Figure 24) [190], the inflection points At $\sim 225,420$ and $500{ }^{\circ} \mathrm{C}$ indicate the temperatures at which the materials' most significant transformations occur. The inflection at $225^{\circ} \mathrm{C}$ may be attributed to the evaporation of organic species, whereas those at 420 and 500 ${ }^{\circ} \mathrm{C}$ correspond to the onset and end of ZnO crystallization, respectively[200]. Accordingly, samples were 
prepared and sintered at slightly higher temperatures to study their microstructural evolution as the sintering temperature was increased.

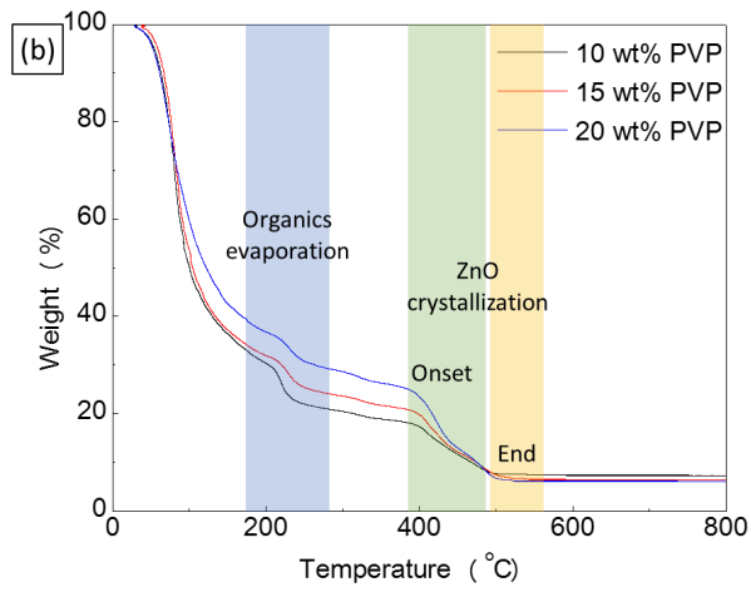

Figure 24.TGA profiles of Al-doped ZnO inks with 10, 15, and 20 wt\% PVP content.

XRD and SEM studies of such samples (see Figure 25), reveal important mechanistic information that further clarifies the microstructural evolution of the AZO structures. Starting at $250^{\circ} \mathrm{C}$, intensity for the $(10 \overline{1} 0)$ and $(10 \overline{1} 1)$ reflections can be observed. At this temperature, the broad peaks indicate the formation of the initial $\mathrm{ZnO}$ nuclei. Diffraction from additional planes is observed to become more significant than the initially observed for the seed crystallites at higher temperatures (i.e. 450 and $525^{\circ} \mathrm{C}$ ), as in the case of the (0002) peak. The microstructural evolution suggests the favoring of the rearrangement of the crystals in the (0002) direction as more energy is available to the system, as observed from the strengthening of the (0002) reflection intensity with increasing temperature. The morphological changes accompanying the crystallographic evolution of the AZO structures, indicate increase in the wrinkling of the films as the processing temperature is increased. The arrows in Figure 25, indicate some of the wrinkle features formed at $450^{\circ} \mathrm{C}$; such wrinkling is more pronounced for the samples treated at $525^{\circ} \mathrm{C}$. These observations, together with those from the XRD studies, suggest the wrinkling to be characteristic of further (0002) crystalline oriented films.
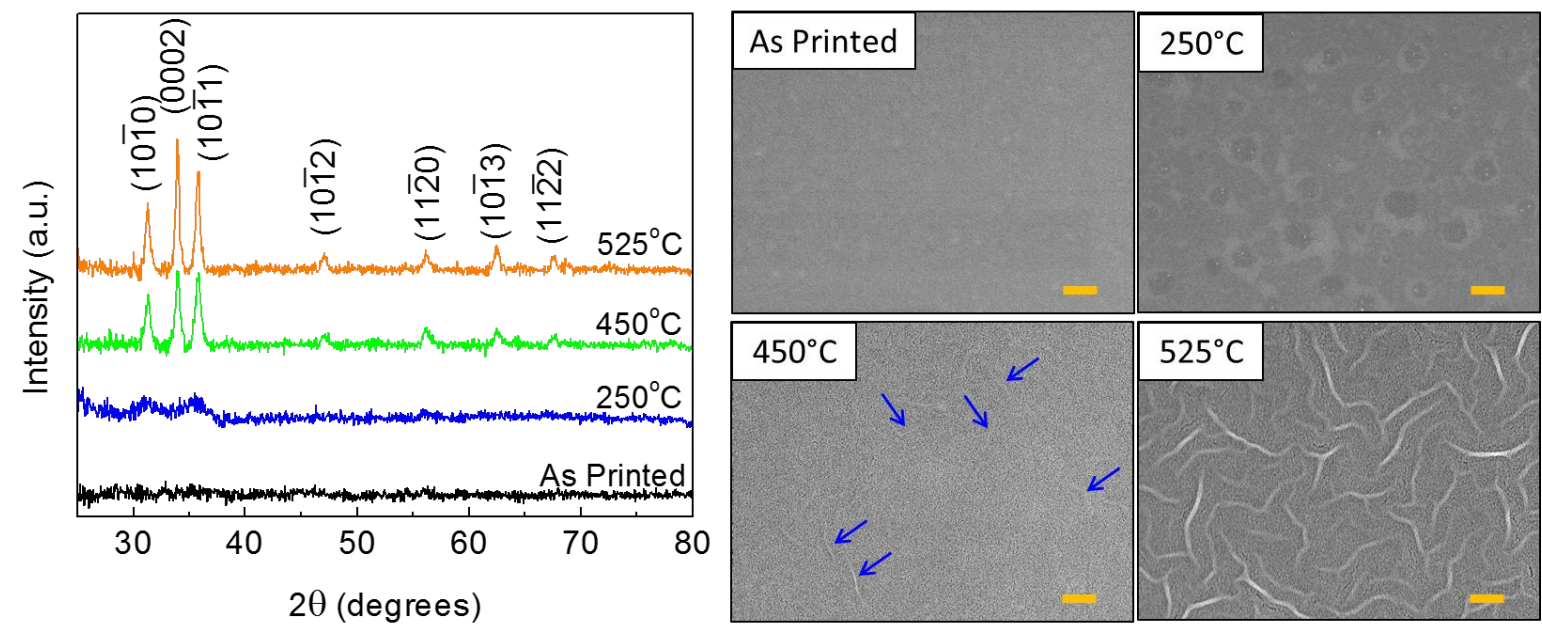

Figure 25. XRD patterns and SEM images of the AZO $15 \mathrm{wt} \%$ structures printed on glass at $3 \mathrm{~mm} / \mathrm{s}$ and $103 \mathrm{kPa}$, and annealed for $20 \mathrm{~min}$ at different temperatures. Arrows indicate wrinkle features; scale bars correspond to $1 \mu \mathrm{m}$. 
The changes in the crystal structure upon different annealing conditions suggest that the wrinkling of the films results from the combination of the stress release from the lattice structure, and from the macroscopic volumetric change of the film, as the organics in the inks are removed. Wrinkling morphologies found in diamond-like films[201] suggest that internal stresses during film formation play important roles in the final morphology. The calculated lattice parameters from the XRD data taken at the different annealing stages, show a systematic decrease in $c$ of $5.78 \%$ from 250 to $450{ }^{\circ} \mathrm{C}$, and of $6.10 \%$ from 250 to $525^{\circ} \mathrm{C}$; while parameter $a$, exhibits increase of $0.30 \%$ from 250 to $450{ }^{\circ} \mathrm{C}$, and of $0.27 \%$ from 250 to $525^{\circ} \mathrm{C}$. See Table 8.

Table 8. Lattice parameters for the AZO (Alo.016Zno.984O) structures from 15 wt\% PVP inks at different annealing temperatures.

\begin{tabular}{lccc}
\hline $\mathbf{1 5} \mathbf{~ w t} \%$ PVP & $\boldsymbol{c}(\AA)$ & $\boldsymbol{a}(\AA)$ & $c / a$ Ratio \\
\hline $\mathbf{2 5 0}{ }^{\circ} \mathbf{C}$ & 5.5554 & 3.2770 & 1.6953 \\
$\mathbf{4 5 0}{ }^{\circ} \mathbf{C}$ & 5.2345 & 3.2866 & 1.5927 \\
$\mathbf{5 2 5}{ }^{\circ} \mathbf{C}$ & 5.2165 & 3.2857 & 1.5876 \\
\hline
\end{tabular}

The proposed film formation mechanism is shown in Figure 26. The formation of nuclei initiates during sintering, at the films' surface - forming a 'crust'- where the heat and mass exchange rate are maximum; and subsequently grow into crystals (Stage A). Additionally, the high molecular weight PVP chains may serve as matrix for nuclei formation, as it has been reported that polymers may be used as organic interface matrices for the heterogeneous crystallization of $\mathrm{ZnO}[202]$. The growth initiates in the (1010) and (10 $\overline{1} 1$ ) directions (Stage B). The accompanying increase in volumetric contraction as sintering continues, induces the wrinkling of the formed "ZnO crust" (Stages C-D), yielding different "wrinkledensities" (number of wrinkle features per unit area) depending on the overall volumetric change. Later, the contacts among the grains (as crystal growth progresses and more energy is available) favor the growth in the (0002) direction, and the next available low energy directions (Stage D). Thus, the direct writing of AZO structures using high volumetric rates (resulting in widths larger than $\sim 250 \mu \mathrm{m}$ ), results in large volumetric contraction of the films during sintering inducing the "wrinkling" of the deposited films, and favoring the stabilization of crystalline orientations different than the expected low surface energy (0002). 


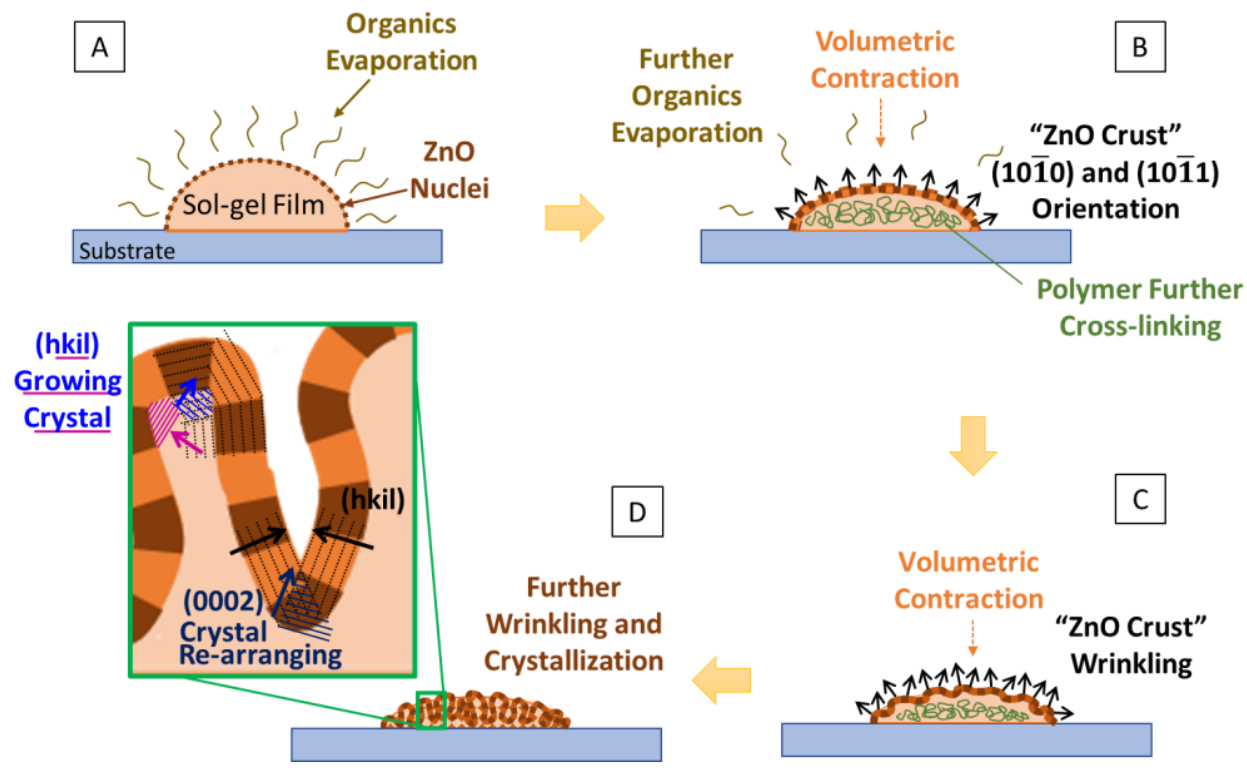

Figure 26 Morphological and crystalline evolution schematic of the directly written Al-doped ZnO films.

From the conducted microstructural studies, it is observed that as less material is printed, more texturing along the (0002) direction is induced; the latter occurring due to the rearrangement of the crystalline planes of the densifying film from (1010) and (10 111$)$ directions, and triggered by temperature during the sintering process. In simpler terms, when less material is printed, the organic molecules are heated and decomposed more efficiently than for bulky prints (transforming a higher amount of ink with the same amount of energy). Correspondingly for finer prints, more energy is available for the crystal rearrangement in the (0002) direction, once the organic decomposition is finished. Therefore, it is postulated that further control of the microstructure is achievable, by controlling the sintering conditions to realize highly wrinkled morphologies (from structures printed using high volumetric flow rates), and exhibiting highly (0002) textured microstructures. To illustrate the potential of this point, one could consider possible the fabrication of highly (0002) textured materials by controlling the sintering conditions to induce the rearrangement of the crystalline planes along such direction. This implies that, the complete rearrangement of the crystals forming the film is possible, provided that there is sufficient energy; and that the wrinkled morphology of the films (dependent on the printing conditions), will thereafter be independent of the heat treatment - having reached the maximum volumetric contraction (i.e. minimum sintered volume). Studies of the heat treatments to define energetic threshold values for attaining fully (0002) textured films, will provide further information about the morphology-texturing relationships.

Additionally, the work by Abidakun proposes that the crystallite size in these structures, is inversely proportional to the amount of printed material, hypothesizing that more nuclei are formed for samples printed with larger amounts of the inks; such films were also demonstrated to exhibit higher wrinkling. From the observations drawn from the analysis of the microstructural evolution (Figure 25), and considering that further wrinkling may lead to higher porosity, a different explanation is proposed; which adding to the observations about more efficient organics decomposition for prints with less amount of material, takes into account the favoring of the grain growth for films with higher packing (less wrinkling). It is, the crystallite size is inversely proportional to the amount of printed material for the sintering 
conditions studied, since the energy provided for such conditions is used to transform different amounts of materials in each case.

\subsection{Illustration of Films Mechanical Flexibility}

Assessment of the mechanical flexibility of these printed systems is interesting from the manufacturing perspective, since materials are often subjected to bending and tensile stresses in the manufacture of devices. Therefore, AZO structures were printed on flexible glass from the 15wt\% PVP formulation and subjected to bending as shown in Figure 27. At an approximate radius of curvature of $69.6 \mathrm{~mm}$, cracks are observed to initiate on the surface of the printed films, upon the application of external bending stress. These cracks propagate perpendicular to the printed structures writing direction, that coincides with the films' longitudinal axis. The AZO structures are subjected to tension by being on the convex side of the glass substrate. Figure 27 (f) indicates delamination at the edges of the crack which may have initiated brittle film cracking. The lower and upper edges of the crack shown in Figure 27, may suggest that the crack propagation occurs from the top surface of the films towards the AZO film/substrate interface and across the width of the printed features.

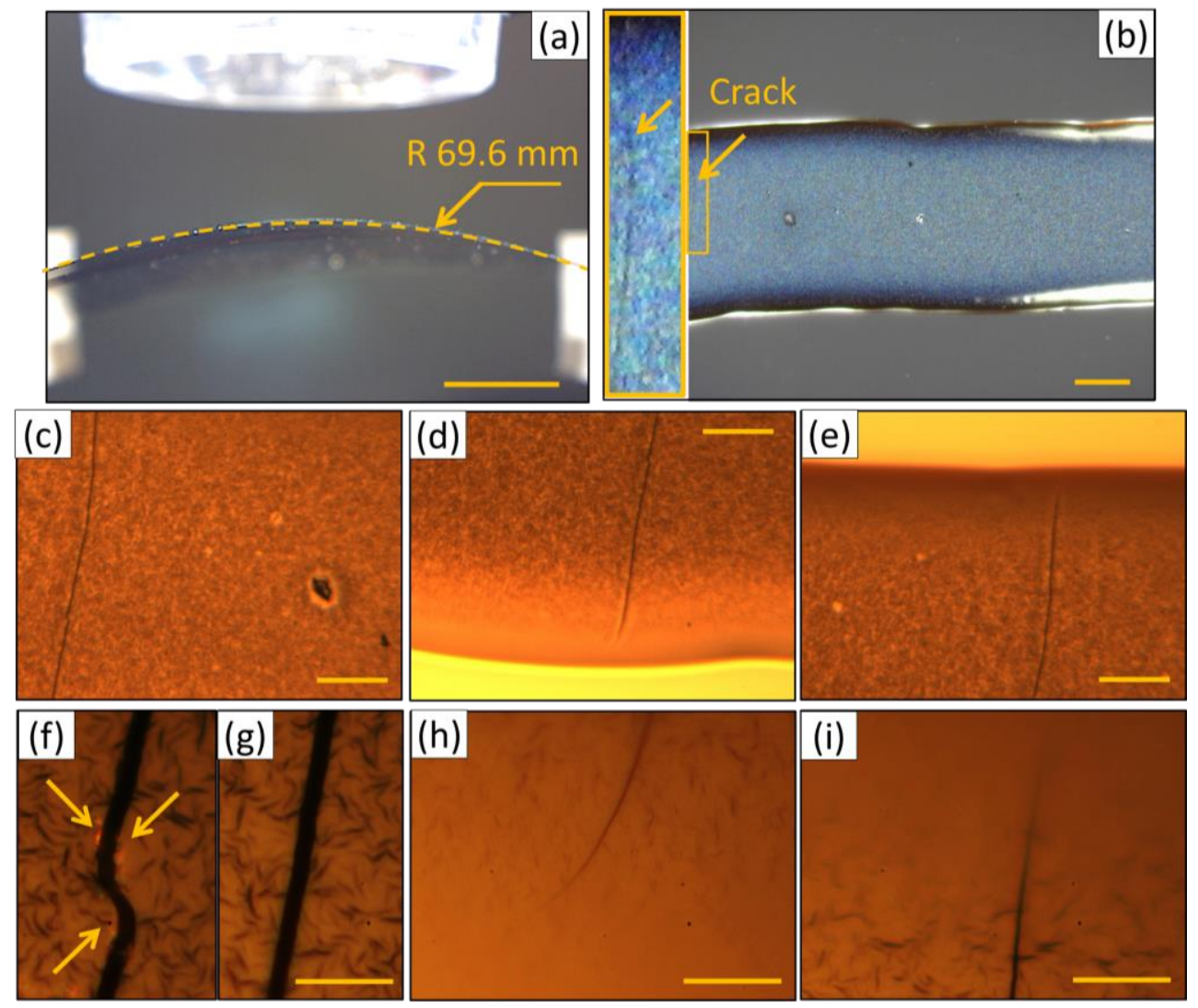

Figure 27. Mechanical testing and optical microscopy images of a typical AZO structure from 15 wt\% PVP ink. (a) Side-view of film during bending; scale bar is $10 \mathrm{~mm}$. (b) Top-view of the film surface showing the appearance of a crack perpendicular to the printing direction (and applied bending stress) at $69.6 \mathrm{~mm}$ radius; scale bar corresponds to $200 \mu \mathrm{m}$. (c-i) Higher resolution images of the region showing the crack. Images (d) and ( $h$ ) exhibit the lower end; and images (e) and (i) the upper end of the crack. Scale bars (c-e) and (f-i), are $100 \mu \mathrm{m}$ and $20 \mu \mathrm{m}$ respectively. Arrows in (f) indicate delamination sites. 


\subsection{Concluding Remarks}

A systematic study of the main factors influencing the texturing of Al-doped $\mathrm{ZnO}$ structures from sol-gel inks using additive manufacturing (printing) has been performed; unveiling their texturing and accompanying morphological evolution mechanisms. From such studies, it is observed that as less material is printed, more texturing along the (0002) direction is induced; the latter occurring due to the more pronounced rearrangement of the crystalline planes of the densifying film with initial $(10 \overline{1} 0)$ and $(10 \overline{1} 1)$ nuclei, and triggered by temperature during the sintering process. Additional aspects of the investigated metal-oxide sol-gel ink system include rheological behavior, ink-substrate interactions, and flexibility assessment.

Further studies relating the surface wrinkling with the different flexibility degrees and /or the wear of these structures, may be of interest for their application in electronic devices subjected to mechanical stresses. Along the same line, the effects of the films' wrinkling on their optical properties is also identified as an interesting research area. In particular, the refractive index of the films may change depending on the wrinkling density and periodicity; nevertheless, it is out of the scope of this thesis and is left as future work. 


\section{Chapter 5: $\mathrm{TiO}_{2}$ : TALH System}

Mesoporous $\mathrm{TiO}_{2}$ films that combine high surface area and efficient electron transport are desired to maximize their performance, allowing more efficient photoexcitation/mass-transport dynamics as device' components such as dye-sensitized solar cells, $\mathrm{H}_{2}$ generation anodes, or photocatalytic reactors. The use of hybrid organic/inorganic systems as for these type of materials synthesis gains interest mainly due to their effect on increasing the electron transport properties $[60,64,203,204]$. Hybrid systems are thought to form continuous-mesoporous architectures by forming semi-crystalline structures that bridge neighboring crystals with the Ti-organics in between, as shown in Figure 28 where the TALH (organic Tiprecursor) forms bridging structures that connect the primary $\mathrm{TiO}_{2}$ particles.

Different polymer agents are included for stabilization of these colloidal systems and control/modification of the ink-substrate interactions. The inks' primary constituents include inorganic $\mathrm{TiO}_{2}$ nanoparticles (main phase), a Ti-organic precursor for $\mathrm{TiO}_{2}$ bridge formation, polymeric agents, and a solvent. The presence of polymeric agents serves different purposes, as rheological properties modulators by modifying the ink's viscosity, and also by influencing the ink substrate interactions. The use of polymer additives has also been reported to prevent clogging of the printing heads when using ink-jet printing, to control the evaporation rate and to reduce cracking of the printed material[88].

Preliminary attempts for formulation of hybrid systems using a TIAA-based sol-gel formulation[124,125] in combination with $\mathrm{TiO}_{2}$ particles, bring up important thermodynamics of mixing issues occurring when utilizing this fast-hydrolyzing compound, and underline the need for water compatible and stable materials systems. See Appendix B.

From the different formulations prepared varying the $\mathrm{TiO}_{2}$ particle amount (TIAA:TiO $, 1: 1$ and 1:2 mol ratio), and polymer agents (PAA and PVP), the main challenge encountered was the rapid sedimentation of organic $\mathrm{TiO}_{2}$ crystals restricting their manipulation in open-air conditions, the different polymers affect the hydrolysis rate of the TIAA to a greater or lesser extent; however, the inks' rheological properties changed within minutes from opening the vials (where the inks were prepared), resulting in spontaneous solidification and clogging of the nozzles for CDW. For the TIAA-PVP formulation without $\mathrm{TiO}_{2}$ primary particles (the most stable amongst the TIAA-based systems), spontaneous crystallization occurred approximately within 6-24 hours after the inks were prepared. Additionally, it was observed that the hydrolyzation behavior was accelerated as larger amounts of $\mathrm{TiO}_{2}$ particles were introduced, suggesting potential relationships with the system surface area $\left(\mathrm{TiO}_{2}\right.$ particles surface area), and potentially physisorbed water at the $\mathrm{TiO}_{2}$ particles' surface.

\section{1. $\mathrm{TiO}_{2}-\mathrm{TALH}$ Ink}

Titanium (IV) bis(ammonium lactato) dihydroxide (TALH), is a water stable Ti-organic precursor that hydrolyses at slow rates in $\mathrm{pH}$ neutral solutions[57,205], avoiding the rapid crystallization issues experienced with Ti-alkoxides when exposed to water in trace amounts or ambient conditions. Previous use of TALH-TiO ${ }_{2}$ formulations for dye-sensitized solar cells $[60,65,206]$ suggest alternative routes for lowtemperature polycrystalline $\mathrm{TiO}_{2}$ film fabrication. Nevertheless, the utilization of the $\mathrm{TALH}-\mathrm{TiO}{ }_{2}$ system has been limited to more traditional deposition / printing methods such as DB[60] and ink-jet printing[65]. 
The implementation of this material system with non-traditional additive manufacturing techniques, such as CDW, serves as motivation to explore these environmentally friendly formulations in different viscosity and printing regimes. Table 9 summarizes the $\mathrm{TiO}_{2}-\mathrm{TALH}$ ink components.

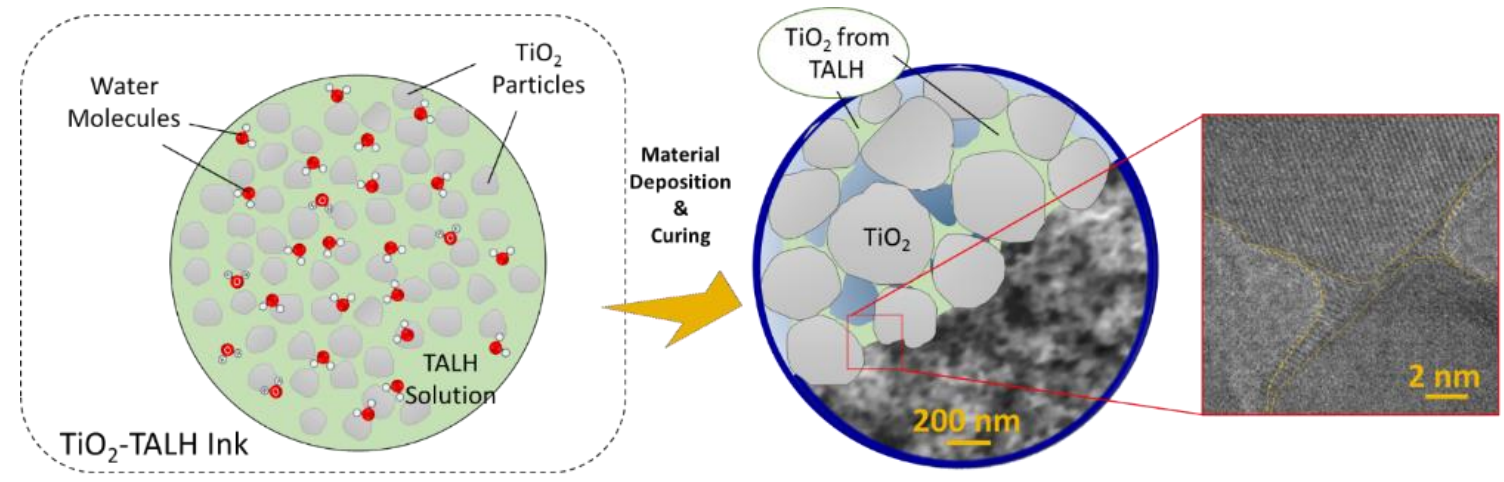

Figure 28. $\mathrm{TiO}_{2}$ - TALH ink system.

Table 9. $\mathrm{TiO}_{2}$-TALH inks precursor table.

\begin{tabular}{|c|c|c|c|c|}
\hline Precursor & Function & Chemical Formula & Chemical Structure & $\begin{array}{c}\text { Molecular } \\
\text { Weight } \\
\text { (g/mol) }\end{array}$ \\
\hline DI Water & Solvent & $\mathrm{H}_{2} \mathrm{O}$ & & 18.015 \\
\hline $\begin{array}{l}\text { Titanium } \\
\text { bis(ammonium } \\
\text { lactato) dihydroxide } \\
\text { - TALH }\end{array}$ & $\begin{array}{c}\text { Ti-organic } \\
\text { (precursor for } \mathrm{TiO}_{2} \\
\text { bridging structures) }\end{array}$ & $\begin{array}{c}{\left[\mathrm{CH}_{3} \mathrm{CH}(\mathrm{O}-\right.} \\
\left.) \mathrm{CO}_{2} \mathrm{NH}_{4}\right]_{2} \mathrm{Ti}(\mathrm{OH})_{2}\end{array}$ & 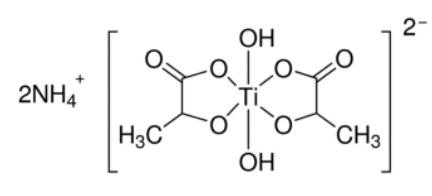 & 294.08 \\
\hline $\begin{array}{l}\text { Titanium Dioxide } \\
\text { Nanoparticles ( } 20 \\
\mathrm{~nm} \text { diameter) - } \\
\text { Aeroxide } \AA\end{array}$ & $\begin{array}{l}\text { Primary particles } \\
\text { (target material } \\
\text { composition) }\end{array}$ & $\mathrm{TiO}_{2}$ & & 79.865 \\
\hline $\begin{array}{l}\text { Polyacrylic Acid - } \\
\text { PAA }\end{array}$ & \multirow{2}{*}{$\begin{array}{c}\text { Rheology enhancer } \\
\text { Nozzle-clogging } \\
\text { preventing }\end{array}$} & $\left(\mathrm{C}_{3} \mathrm{H}_{4} \mathrm{O}_{2}\right)_{\mathrm{n}}$ & & 72.033 \\
\hline $\begin{array}{l}\text { Polyvinylpyrrolidone } \\
\text { - PVP }\end{array}$ & & $\left(\mathrm{C}_{6} \mathrm{H}_{9} \mathrm{NO}\right)_{\mathrm{n}}$ & & 111.084 \\
\hline
\end{tabular}

\subsection{Ink Rheology and Thermal Decomposition}

The different $\mathrm{TALH}: \mathrm{TiO}_{2}$ ratio inks studied, consisting of a TALH $0.4 \mathrm{M}$ aqueous solution and variable amounts of $\mathrm{TiO}_{2}$ nanoparticles as (1:1), (1:3), (1:6) and (1:12) in mol:mol, can also be considered as 
formulations with variable solvent content. Thus, for the lower $\mathrm{TiO}_{2}$ formulations, more solvent is present in proportion to the amount of solids, directly influencing the viscosity of the systems as observed from the viscosity measurements (see Figure 29).

Table 10. TiO2-TAHL inks solids and liquid contents.

\begin{tabular}{lccccc}
\hline $\begin{array}{c}\text { Ink TAHL:TiO } \\
\text { (mol:mol) }\end{array}$ & $\begin{array}{c}\text { TALH } \\
\text { (wt\%) }\end{array}$ & $\begin{array}{c}\mathrm{TiO}_{\mathbf{2}} \text { Solids } \\
\text { (wt\%) }\end{array}$ & $\begin{array}{c}\text { Polymer } \\
\text { (wt\%) }\end{array}$ & $\begin{array}{c}\text { Water } \\
\text { (wt\%) }\end{array}$ & $\begin{array}{c}\mathrm{TAHL:TiO}_{2} \\
\text { (wt\%:wt\%) }\end{array}$ \\
\hline $\mathbf{( 1 : 1 )}$ & 10.950 & 2.970 & --- & 86.080 & 3.687 \\
$\mathbf{( 1 : 3 )}$ & 10.330 & 8.420 & --- & 81.250 & 1.227 \\
$\mathbf{( 1 : 6 )}$ & 9.530 & 15.530 & --- & 74.940 & 0.614 \\
$\mathbf{( 1 : 6 ) ~ P A A}$ & 9.310 & 15.170 & 2.280 & 73.240 & 0.614 \\
$\mathbf{( 1 : 6 ) ~ P V P}$ & 9.200 & 14.990 & 3.470 & 72.340 & 0.614 \\
$\mathbf{( 1 : 1 2 )}$ & 8.250 & 26.880 & --- & 64.870 & 0.307 \\
$\mathbf{( 1 : 1 2 ) ~ P A A ~}$ & 8.090 & 26.350 & 1.980 & 63.580 & 0.307 \\
$\mathbf{( 1 : 1 2 ) ~ P V P}$ & 8.000 & 26.070 & 3.020 & 62.910 & 0.307 \\
\hline
\end{tabular}
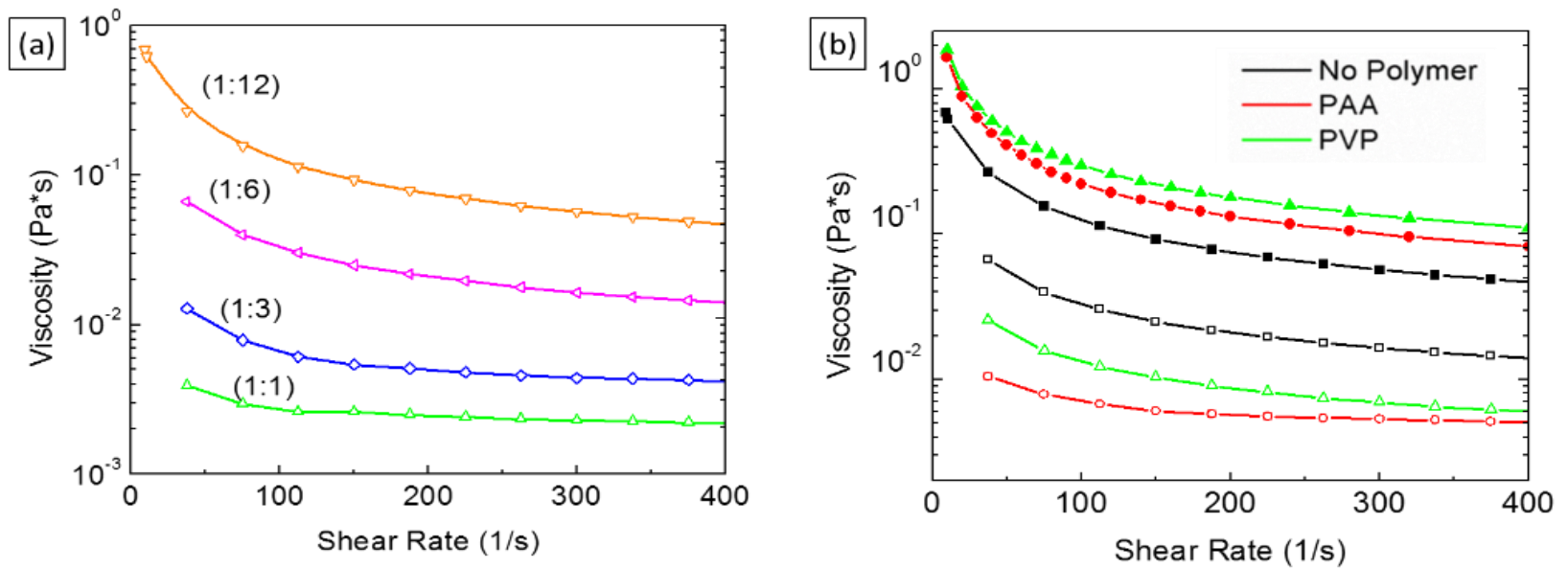

Figure 29. Viscosity of TALH:TiO 2 systems (a) without polymers and (b) with polymers; open symbols correspond to (1:6), and filled symbols to (1:12) formulations respectively.

The formulated colloidal systems exhibit a strong particle aggregation tendency which is evident from the sedimentation of the inks with lower viscosity and $\mathrm{TiO}_{2}$ content (1:1), (1:3) and (1:6). As polymer additives are incorporated, more stable formulations are obtained reducing clogging of the nozzles when utilizing robotic deposition (1:6 and 1:12 inks). The lower viscosity inks are especially suitable for ink-jet printing $[84,88]$; whereas the consistency of the $1: 12$ formulation resembles that of a slurry, and is suitable for CDW and screen printing.

A slight viscosity decreasing effect of the viscosity of the 1:6 inks as polymers are added to the system, in contrast to the increasing effect exhibited for the 1:12 polymer formulations, and is attributed to the combination of a reduction of the viscous forces between the $\mathrm{TiO}_{2}$ particles, and the swelling of the added polymers in the liquid phase of the inks. 
The drying characteristics of these ink systems are strongly affected by the amount of solids present; i.e. more $\mathrm{TiO}_{2}$ particles imply larger surface area for the solvent to adsorb and to evaporate. As the inks are deposited onto the substrates, the aggregation of the particles at the center, characteristic of Marangoni flow [207], is more pronounced for the lower viscosity inks with more free volume for the colloids to move. This in turn, leads to non-uniform films (thicker and rougher at the center, and thinner and smother towards their periphery). This drying behavior is common to the two deposition techniques utilized (DB and CDW); as the substrate area wetted by the ink is reduced, the evaporation rate increases (since there are more drying fronts) and the mobility of the particles in the inks is resulting in less noticeable differences between the inner and outer regions. In Figure 30, the average roughness (measured through profilometry) for the thinner and thicker regions are $6.9 \mu \mathrm{m}$ and $9.6 \mu \mathrm{m}$, respectively.

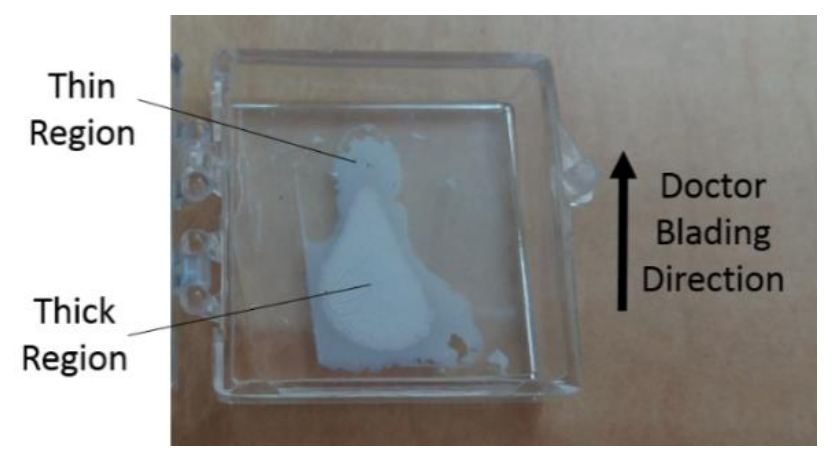

Figure 30. Photograph of a doctor bladed $\mathrm{TiO}_{2}$ film from a low viscosity ink formulation (TALH:TiO 2 1:4.5 no-polymer) exhibiting strong particle aggregation.

Thermo-gravimetric analysis (TGA) of representative formulations, show how the different components in the inks evacuate the system through evaporation or thermal degradation, and also where the material phase transitions may occur. Around $\sim 120{ }^{\circ} \mathrm{C}$, evaporation of the majority of the solvent is observed; the inflection points at $\sim 150^{\circ} \mathrm{C}, \sim 325^{\circ} \mathrm{C}$ and $\sim 425^{\circ} \mathrm{C}$, are attributed to the onset for TALH degradation and $\mathrm{TiO}_{2}$ initial formation, TALH transformation into amorphous $\mathrm{TiO}_{2}$, and amorphous $\mathrm{TiO}_{2}$ transformation into anatase phase, respectively. This typical TGA profile (for all the inks) could be analyzed as the superposition of the TGA curves for the main constituents of the inks. For the TALH 2.08M precursor aqueous solution these inflections are the most pronounced (being an organic compound in solution); whereas the TGA profile for the crystalline phase $\left(\mathrm{TiO}_{2}\right.$ only - Aeroxide $\left.{ }^{\circledR}\right)$ shows very small weight changes as thermal energy is added to the system. The formulated inks, with a TALH concentration of $0.4 \mathrm{M}$, exhibit less pronounced transitions; and due to the presence of already crystalline material $\left(\mathrm{TiO}_{2}\right.$ nanoparticles) further dampening of the magnitude of their change in weight is observed as temperature is increased. 

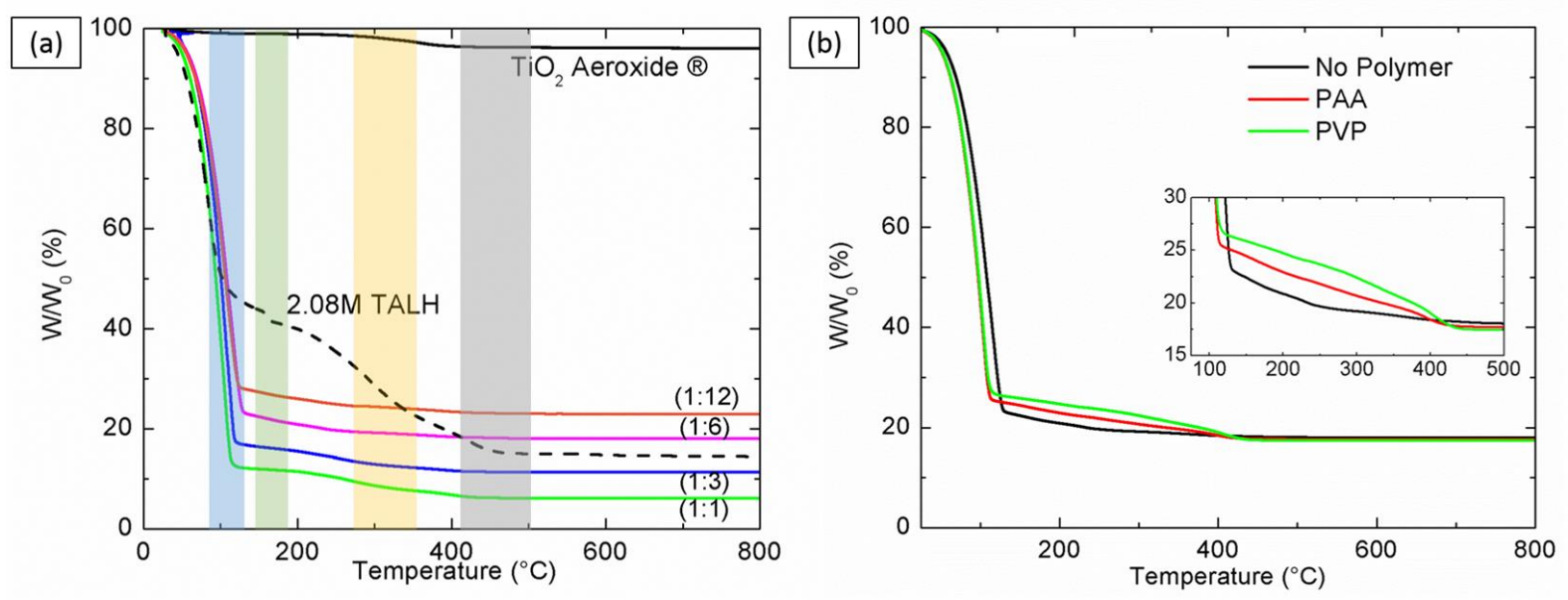

Figure 31. TGA of TALH:TiO2 systems (a) without polymers and for (b) 1:6 different polymer formulations.

A transition at $\sim 400^{\circ} \mathrm{C}$, characteristic of amorphous $\mathrm{TiO}_{2}$ into anatase phase [208] is identified for the bare $\mathrm{TiO}_{2}$ nanoparticles, suggesting the presence of trace amorphous $\mathrm{TiO}_{2}$, since the precursor $\mathrm{TiO}_{2}$ is already in a thermodynamically stable phase (anatase or rutile). As reported in literature, depending on the flame conditions $[209,210]$ used in the Aerosil ${ }^{\circledR}$ (synthesis) process, different amounts of amorphous $\mathrm{TiO}_{2}$ can be expected.

\section{3. $\mathrm{CDW}$ of $\mathrm{TiO}_{2}-\mathrm{TALH}$ Inks}

The viscosity ranges from the studied $\mathrm{TALH}: \mathrm{TiO}_{2}$ formulations, see Figure 29, allow printing of 2D film and pattern structures. The use of polymeric additives reduces significantly the clogging issues when roboticprinting 1:6 and 1:12 $\mathrm{TALH}: \mathrm{TiO}_{2}$ formulations, consequently yielding prints with less defects such as discontinuities or uneven coverage. Additional uses for polymeric agents in inks reported in literature include control of the evaporation rate and nozzle clogging prevention [88]; and films' crack reduction and substrate wetting modification $[60,107,211]$. Nozzle clogging is also caused by particle aggregation in the colloidal systems; among the investigated inks, the 1:12 formulations exhibited the most kinetically stable behavior, resulting in better printability. Inks with lower initial $\mathrm{TiO}_{2}$ content exhibit strong sedimentation and are not suitable for robotic deposition without the use of stabilizing agents. The viscosity of these ink systems plays an additional role in the printing mechanism, as capillary effects are more significant for lower viscosity inks. Consequently, printing of inks with low viscosities (bellow those measured for 1:6 TALH:TiO 2 ), may resemble the ink-flow dynamics of slot-die coating[212] - with the differentiation of CDW allowing the patterning of the inks by having a stage-movement in the $x-y-z$ directions.

The direct writing parameters include ink extrusion pressure, printing nozzle inner diameter, dispensing height (i.e., the nozzle-substrate clearance), and writing speed (speed at which the printing nozzle traverses the substrate surface)[93]. The direct writing ranges found to yield the best quality (i.e. continuous and uniform) and most reproducible films for the 1:12 no-polymer ink, are $55 \mathrm{kPa}$ to $70 \mathrm{kPa}$ pneumatic pressure, $5 \mathrm{~mm} / \mathrm{s}$ to $10 \mathrm{~mm} / \mathrm{s}$ writing-speed, and $\sim 60 \mu \mathrm{m}$ to $80 \mu \mathrm{m}$ dispensing height using a $100 \mu \mathrm{m}$ - nozzle size. As polymers are added to the formulations, the robotic printing spaces broaden into larger printing regions (see Figure 32). 

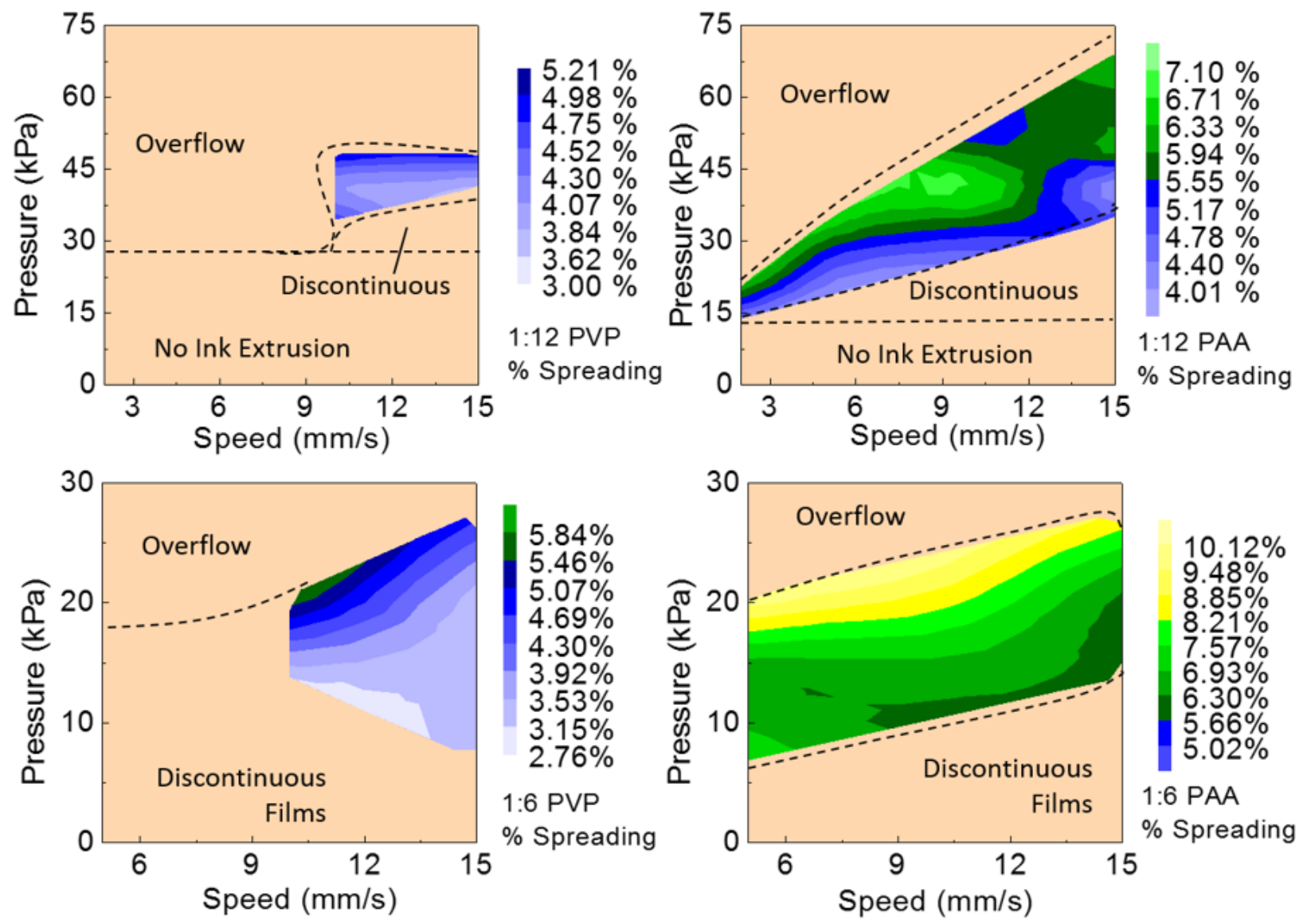

Figure 32. Direct writing parameter maps for the different TALH:TiO 2 polymer added inks on ITO/PET substrates.

The writing speed is observed to be a determining parameter for obtaining relatively uniform and reproducible films and patterns. A relatively slow ( $5 \mathrm{~mm} / \mathrm{s})$ deposition causes the drying front of the films to match the deposition speed yielding uniform printed features. However, if the writing speed is further decreased, the drying front of the deposited material traveling faster than the ink being deposited, results in clogging of the nozzles and discontinuous prints. The concomitant relation between writing speed and applied pressure is also of great importance, since too much pressure at low speed results in overflow of material and thick films and conversely, high speeds at a given pressure result in thinner films and eventually uncovered regions. Additionally, strong $\mathrm{TiO}_{2}$ particle aggregation results in cracking of the films (as they dry) if too much material is extruded over a small area. The pressure-speed printing ranges for 1:6 inks, are smaller because of the lower viscosity and lower kinetic stability of these inks, when compared to their higher initial $\mathrm{TiO}_{2}$ content counterparts.

The nozzle-substrate clearance is another important parameter in consideration; an excessively large distance (at a $90^{\circ}$ angle position between the nozzle axis and the substrate surface), may cause the inks to climb up the nozzle outer walls due to the inks' surface tension. On the other hand, if the nozzle is too close to the substrate, the profile of the deposited material is mechanically altered by the nozzle and may present a "valley-like" shape depending on the ink's elastic properties; furthermore, it will cause larger spreading of the inks and thus reduced printing resolution. The latter may be desirable for the fabrication of films. 
The assessment of the ink-substrate interactions is crucial to address potential problems of new materials systems, such as ink-substrate incompatibility (non-stickiness) and/or material delamination. The spreading of the inks and the thickness and micron-scale roughness of the printed films are found to be strongly related. Increase in the material being deposited (from increase in pressure at a fixed writing speed), results in larger spreading, thicker and rougher prints. Spreading, highly dependent on the inksubstrate interactions as observed from the contact angle results, exhibits larger values for the lower initial $\mathrm{TiO}_{2}$ particle concentration inks (higher solvent content) than for the 1:12 formulations. Typically, 1:12 TALH: $\mathrm{TiO}_{2}$ thick films of $\sim 70 \mu \mathrm{m}$ to $80 \mu \mathrm{m}$ thickness and of $\sim 10 \mu \mathrm{m}$ to $19 \mu \mathrm{m}$ roughness; and thinner films of $\sim 20 \mu \mathrm{m}$ to $30 \mu \mathrm{m}$ thickness and of $\sim 4.5 \mu \mathrm{m}$ to $6.5 \mu \mathrm{m}$ roughness are obtained using CDW. Similarly, 1:6 TALH:TiO ${ }_{2}$ films of $\sim 50 \mu \mathrm{m}$ and $\sim 20 \mu \mathrm{m}$ thickness, exhibit $\sim 20 \mu \mathrm{m}$ and $\sim 5 \mu \mathrm{m}$ roughness, respectively. Additionally, it is found that the different polymers' chemistries greatly influence the inks' spreading/wetting of the substrates. Moreover, the direct writing parameters are observed to be affected by the choice of the polymers included in the formulation; generally, larger successful printing ranges are obtained for the more acidic (PAA) formulations on ITO/PET substrates, in agreement with the contact angle measurements.
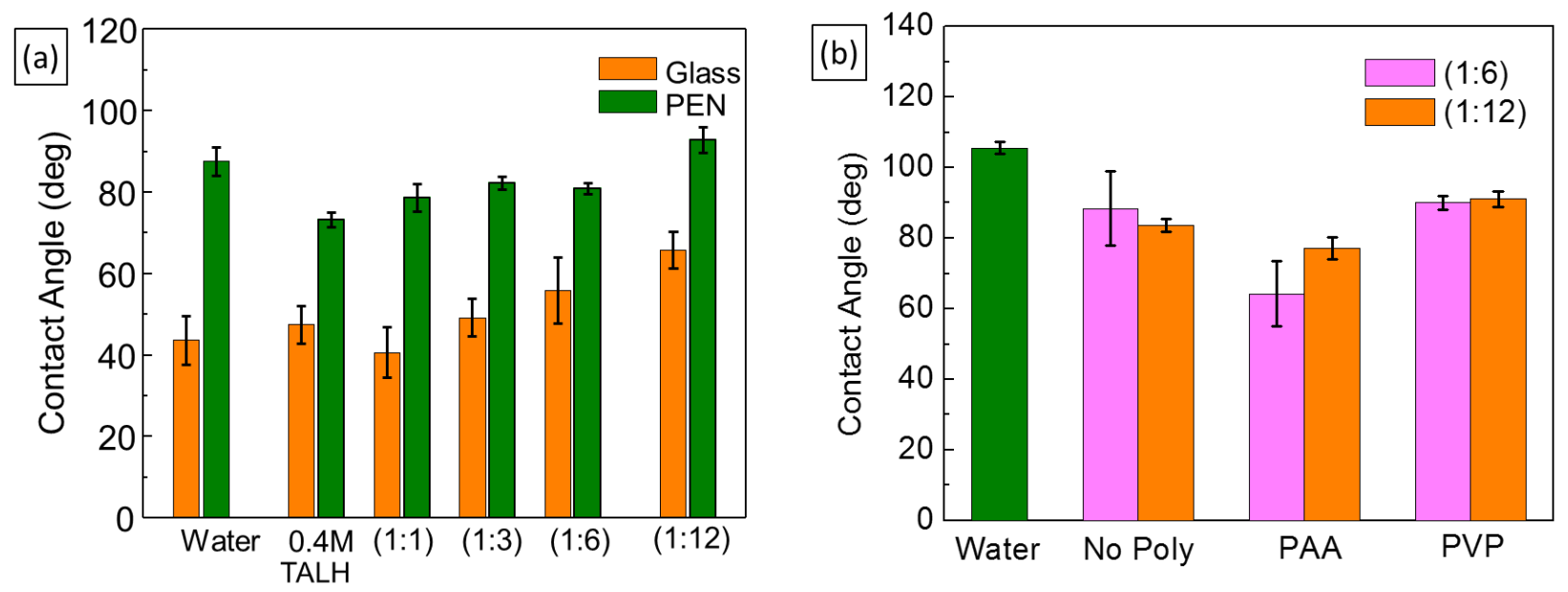

Figure 33. Contact angle of (a) different particle concentration inks on glass and PEN substrates; and of (b) polymer added formulations on ITO/PET substrates.

The microstructure of the prepared samples (Figure 34) show porous particle-aggregate films from all ink formulations regardless of the initial $\mathrm{TiO}_{2}$ particle concentration or the material deposition method (DB or CDW). Though slight reduction of the films' cracking is observed for the polymer formulations, the similarity of the films' surface microstructure is mainly attributed to the strong particle aggregation in the colloidal systems. Electrostatic stabilization of the $\mathrm{TiO}_{2}$ particles may be induced by changing the $\mathrm{pH}$ of the inks. The isoelectric point of $\mathrm{TiO}_{2}$ is 6.25 [213]; thus, the acidic character of the PAA may favor the stabilization of the dispersions compared to PVP (being less acidic). Investigation of the surface charging using z-potential measurements of the primary particles in different $\mathrm{pH}$ suspensions will provide further routes for controlling the aggregation. 


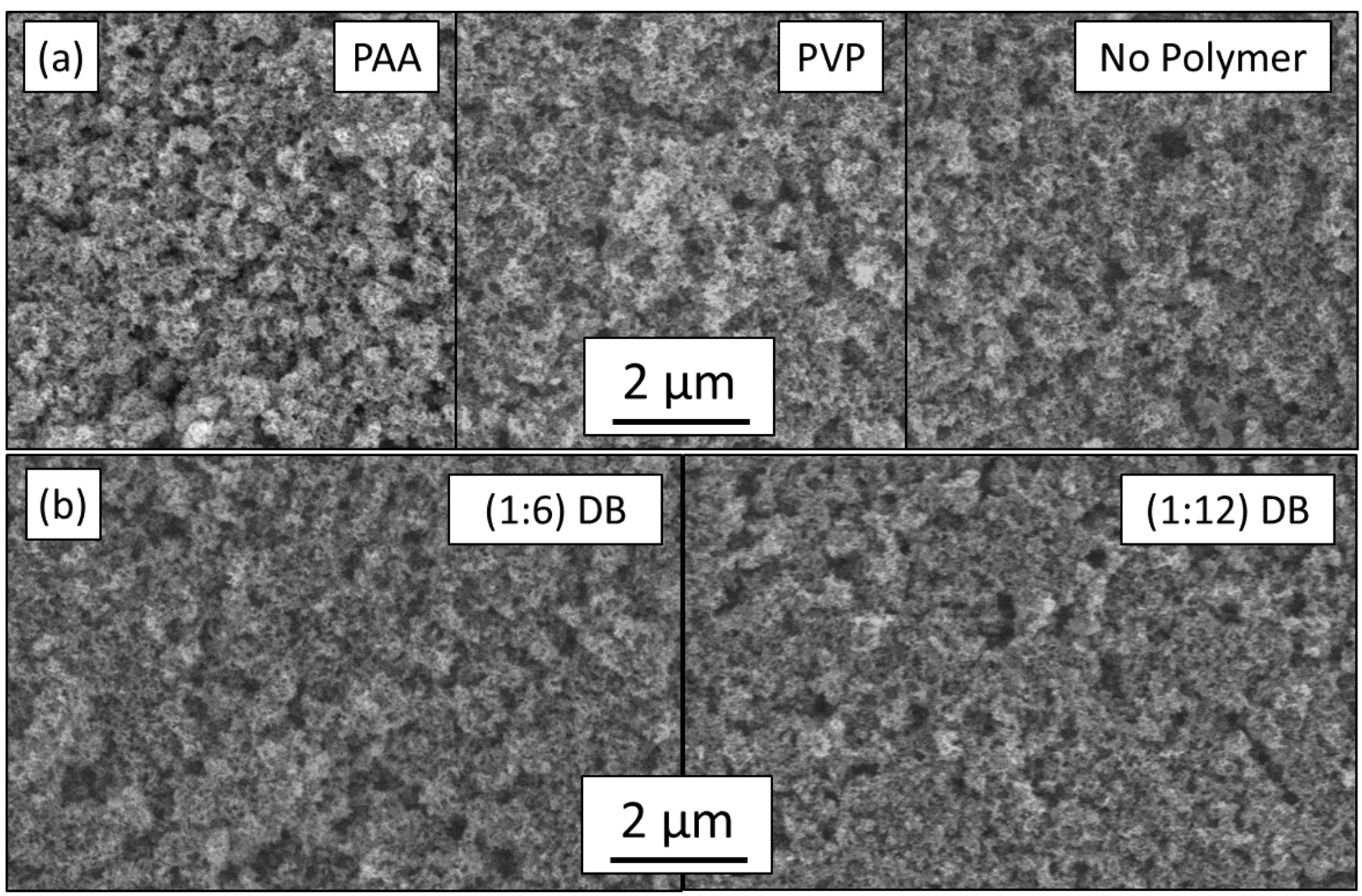

Figure 34. SEM images of the surface of (a) directly written $\mathrm{TiO}_{2}$ films, from $\mathrm{TALH}^{\mathrm{TiO}} \mathrm{Ti}_{2}$ (1:12) precursor inks; and (b) of doctor bladed (DB) films, from (1:6) and (1:12) TALH: TiO 2 formulations on Ag/PEN after $6 h$ UV exposure.

\subsection{Microstructure and Surface Chemistry}

XRD of the obtained films show characteristic diffraction patterns of polycrystalline $\mathrm{TiO}_{2}$ in anatase and rutile phase as expected from the $\mathrm{TiO}_{2}$ particles used for the inks' preparation. Comparative studies of the XRD exhibited by the films with different post-printing energy-treatments, provide insight about the effect of such treatments on the films microstructure. The calculated grain size of the different TALH:TiO films, with and without polymers, suggests important mechanisms of $\mathrm{TiO}_{2}$ nucleation and growth. A systematic peak broadening of the peaks corresponding to (011) Anatase and (110) Rutile as energy is supplied to the system (as heat or UV light) is observed. This broadening indicates smaller crystallite sizes as more energy is delivered to the system, as calculated using Scherrer's equation[183]. 

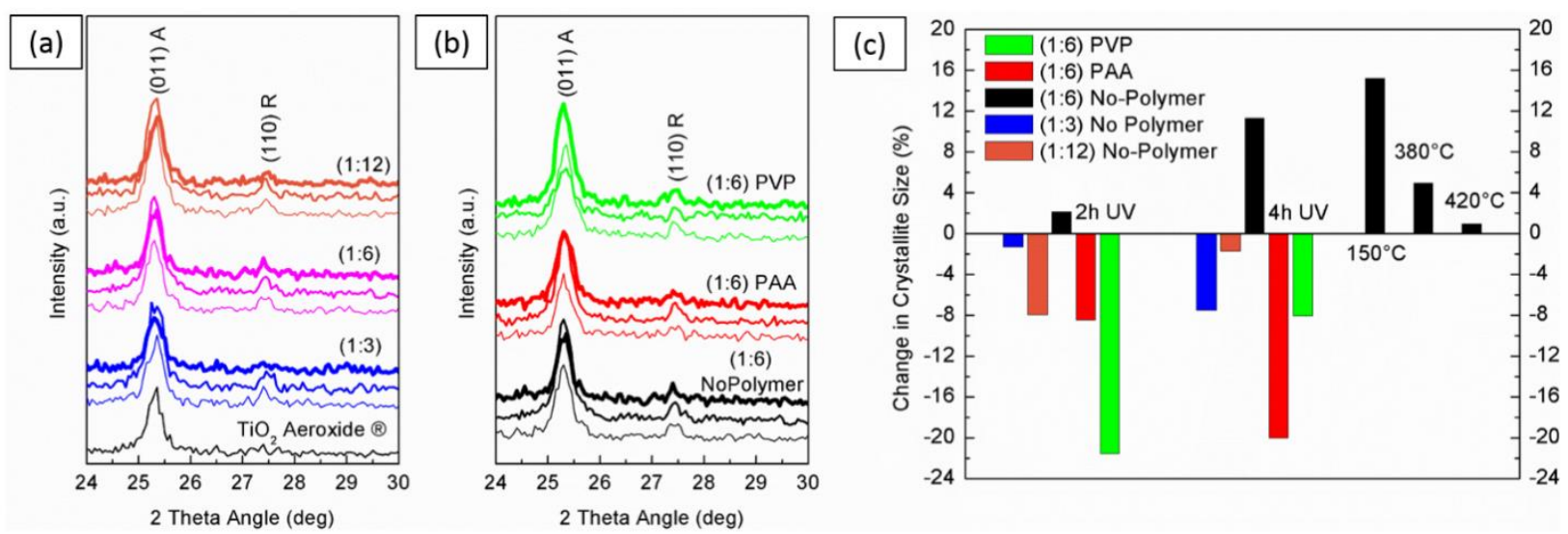

Figure 35. XRD of (a) $\mathrm{TiO}_{2}$ Aeroxide (B) and the different particle concentration films and (b) of the TALH:TiO 2 (1:6) films with various polymers, after different UV treatments: No Treatment (thin-bottom line), $2 \mathrm{~h}$ UV (medium) and 4hUV (dark-top line). (c) Crystallite size change upon different energy input conditions, variation calculated with respect to non-treated samples.

Given the stable nature of the crystalline $\mathrm{TiO}_{2}$, it is hypothesized that the experimentally observed decrease in crystallite size for the polycrystalline films corresponds to new $\mathrm{TiO}_{2}$ crystallites formed from TALH. XRD of the calcined TALH powder sample shows diffraction typical of anatase phase in accordance with PDF-00-021-1272.

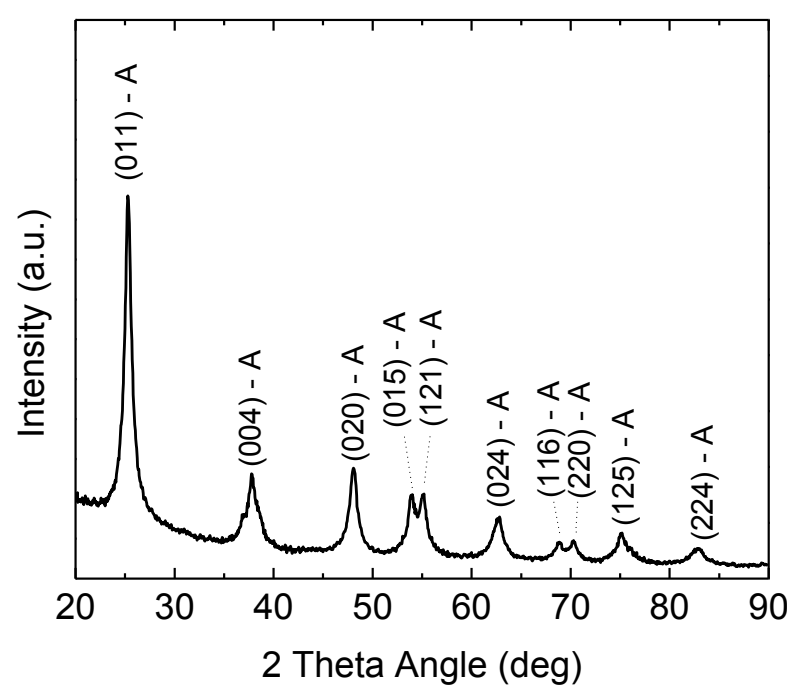

Figure 36. XRD spectrum of the $500^{\circ} \mathrm{C}$ calcined TALH powder sample.

Except for the 1:6 No-Polymer formulations, all the other analyzed films exhibit $\mathrm{TiO}_{2}$ crystallite size decrease upon energy treatments. It is proposed that the reason for such behavior is that the amount of $\mathrm{TALH}$ to $\mathrm{TiO}_{2}$ is optimum for particle growth from the existing $\mathrm{TiO}_{2}$ crystals initially added to the system. In contrast, as polymers are added to the (1:6) formulation, this trend is reversed and smaller crystallite sizes are obtained.

Two possible sites for $\mathrm{TiO}_{2}$ nucleation are, either at high $\mathrm{Ti}$ and $\mathrm{O}$ concentration regions within the liquid phase (in solution), or by self-assembling onto the already ordered $\mathrm{TiO}_{2}$ surfaces from the initially added $\mathrm{TiO}_{2}$ particles. As UV exposure is increased from 0 to 4 hours, the diffraction peaks corresponding to the anatase and rutile phases broaden, suggesting that the former crystallization mechanism dominates. On 
the other hand, when thermal energy is supplied to the system (1:6 case), a maximum for the $\mathrm{TiO}_{2}$ crystallite size at $\sim 150^{\circ} \mathrm{C}$ is observed which may be indicative of growth on existing $\mathrm{TiO}_{2}$ surfaces. Further thermal energy input to the system results in crystallization of the remaining $\mathrm{Ti}$ and $\mathrm{O}$ from the solution as individual $\mathrm{TiO}_{2}$ particles, as suggested by the systematic decrease in crystallite size from $150^{\circ} \mathrm{C}$ to $420^{\circ} \mathrm{C}$ heat treatment.

During deposition and treatment of the $\mathrm{TAHL}: \mathrm{TiO}_{2}$ films produced in the current investigation, there are not direct removal mechanisms for the ammonium lactate present (other than thermal or UV-light decomposition), therefore small $\mathrm{TiO}_{2}$ crystallites from $\mathrm{TALH}$ can be expected; since the ammonium lactate ions present, as by-product of TALH hydrolysis, act as a capping layer hindering $\mathrm{TiO}_{2}$ crystallite growth. Neutralization or removal of the ammonium lactate during the synthesis of $\mathrm{TiO}_{2}$ form TALH under hydrothermal conditions, indicates that Oswald ripening is prevented by ammonium lactate[205].

Raman spectroscopy (Figure 37) shows the typical mixed phase $\mathrm{TiO}_{2}$ (anatase/rutile) pattern with energy bands in the inorganic region [214-216]. Also in the organic region, $700 \mathrm{~cm}^{-1}$ to $200 \mathrm{~cm}^{-1}$ Raman shift, the ammonium lactate characteristic spectrum is observed [217]; Table 11 contains the detailed Raman band - vibrational modes assignment for the prepared films. The anatase to rutile ratio, as calculated from the ratio of the integrated areas of the 395 - Anatase and 445 - Rutile peaks[218], is indicative of the transformation of the films when treated with UV-light or temperature. All the obtained spectra were slightly displaced towards higher energy shift values from 1 to $3 \mathrm{~cm}^{-1}$, due to the experimental error associated with the equipment and temperature fluctuations, and the presence of organic molecules adsorbed onto the $\mathrm{TiO}_{2}$ surface[219].
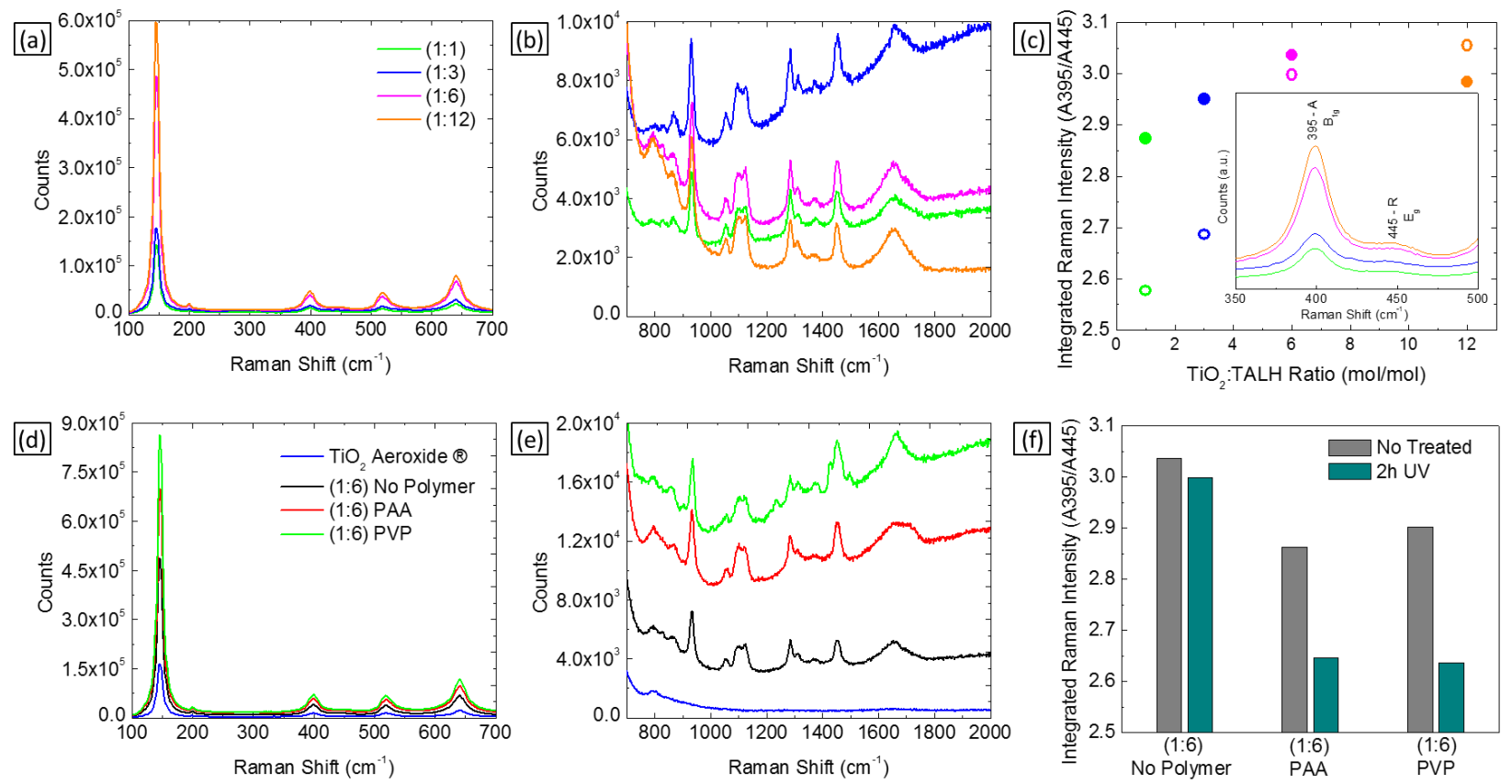

Figure 37. Visible Raman $\lambda=532 \mathrm{~nm}$ for the as dried (No treatment) TALH:TiO 2 inks (a) inorganic region (b) organic region, as dried (No treatment) different polymer formulated inks (d) inorganic region and (e) organic region. Integrated Raman Intensity ratios for (c) different particle loading inks and (f) different polymer formulated inks as dried and after 2 h UV treatment. Open Symbols (No Treatment), filled symbols (2hUV). Inset in (c) depicts the integration areas: Anatase $\left(395 \mathrm{~cm}^{-1}\right)$ and Rutile $\left(445 \mathrm{~cm}^{-1}\right)$ peaks. 
Table 11. Experimental (average) Raman band positions obtained for the different TALH:TiO films. $^{2}$

\begin{tabular}{lcc}
\hline \multicolumn{1}{c}{ Vibrational Mode } & $\begin{array}{c}\text { Experimental Average Peak } \\
\text { Position }\left(\mathbf{c m}^{-1}\right)\end{array}$ & Reference \\
\hline $\mathrm{B}_{1 \mathrm{~g}}$-Rutile or $\mathrm{E}_{\mathrm{g}}$-Anatase & 145.8 & {$[214,215]$} \\
$\mathrm{E}_{\mathrm{g}}$-Anatase & 199.6 & {$[214,215]$} \\
O-O interactions & 267.6 & {$[216]$} \\
O-O interactions & 297.2 & {$[216]$} \\
$\mathrm{B}_{1 \mathrm{~g}}$-Anatase & 398.1 & {$[214,215]$} \\
$\mathrm{E}_{\mathrm{g}}$-Anatase & 449.1 & {$[214,215]$} \\
$\mathrm{B}_{1 \mathrm{~g}}$-Anatase $([214]) ;$ A1g-Anatase ([215]) & 518.7 & {$[214,215]$} \\
$\mathrm{E}_{\mathrm{g}}$-Anatase ([214]); B1g-Anatase ([215]) & 639.2 & {$[214,215]$} \\
Anatase Combination band & 794.2 & {$[214]$} \\
\hline
\end{tabular}

The calculated amount of anatase and rutile phase from XRD[208] and Raman[218], see Table 12, show differences that can be attributed to several factors involved in the data collection and analysis, including the resolution from the XRD patterns and Raman spectra, the experimental set-up and the precision of the fitting (when integrating the peak areas). Nevertheless, the results obtained from these two techniques agree by showing an increasing (or decreasing) trend that holds for both technique results, qualitatively validating the changes induced to the $\mathrm{TiO}_{2}-\mathrm{TAHL}$ system. Because XRD consists of a statistical technique that averages the collected signal over a larger amount of material, when compared to the actual sample region ( $\sim 50 \mu \mathrm{m}$ diameter) probed for Raman acquisition, XRD would give a better quantitative analysis of the phase ratios. 
Table 12. Calculated amount of anatase and rutile (wt.\%) from the Raman and XRD data for the different deposited films.

\begin{tabular}{|c|c|c|c|c|}
\hline \multirow[b]{2}{*}{ Film TALH:TiO2 ratio } & \multicolumn{2}{|c|}{ Raman } & \multicolumn{2}{|c|}{ XRD } \\
\hline & Anatase wt\% & Rutile wt\% & Anatase wt\% & Rutile wt\% \\
\hline (1:3) No Treatment & 90.08 & 9.92 & 79.76 & 20.24 \\
\hline (1:3) 2hUV & 90.94 & 9.06 & 87.36 & 12.64 \\
\hline (1:3) 4h UV & --- & ---- & 79.83 & 20.17 \\
\hline (1:6) No Treatment & 91.08 & 8.92 & 82.69 & 17.31 \\
\hline$(1: 6) 2 \mathrm{hUV}$ & 91.19 & 8.81 & 89.13 & 10.87 \\
\hline (1:6) $4 \mathrm{~h}$ UV & ---- & ---- & 86.42 & 13.58 \\
\hline (1:6) PAA No Treatment & 91.11 & 8.89 & 85.44 & 14.56 \\
\hline (1:6) PAA 2h UV & ---- & ---- & 86.67 & 13.33 \\
\hline (1:6) PAA 4hUV & ---- & --- & 84.89 & 15.11 \\
\hline (1:6) PAA 6hUV & 91.47 & 8.53 & ---- & ---- \\
\hline (1:6) PAA $150^{\circ} \mathrm{C}-30 \mathrm{~min}$ & 90.84 & 9.16 & ---- & ---- \\
\hline (1:6) PVP No Treatment & ---- & ---- & 90.85 & 9.15 \\
\hline (1:6) PVP 2h UV & ---- & ---- & 85.17 & 14.83 \\
\hline (1:6) PVP 4h UV & ---- & ---- & 87.21 & 12.79 \\
\hline (1:12) No Treatment & 89.68 & 10.32 & 88.31 & 11.69 \\
\hline$(1: 12) 2 \mathrm{hUV}$ & 91.03 & 8.97 & 89.87 & 10.13 \\
\hline (1:12) 4hUV & & & 87.78 & 12.22 \\
\hline $\mathrm{TiO}_{2}$ Aeroxide ${ }^{\circledR}$ & ---- & ---- & 79.07 & 20.93 \\
\hline
\end{tabular}

The $\mathrm{TiO}_{2}$ phase transitions are strongly influenced by the crystallite size. For $\mathrm{TiO}_{2}$ crystallites of up to 2.8 $\mathrm{nm}$ size, anatase is a more thermodynamically stable phase than rutile because of their respective surface energies; later as the crystallites grow, the Gibbs free energy associated to the crystal volume for rutile is smaller than that for the anatase phase and therefore the rutile structure is ultimately the most stable of the $\mathrm{TiO}_{2}$ phases[208]. This is very likely to be the reason for anatase to be the first of the phases to form as Ti-organic precursors transform into $\mathrm{TiO}_{2}$. In addition to this, the anatase to rutile phase transformation is highly dependent on the crystallite size of the $\mathrm{TiO}_{2}$ primary particles; initiating at the interfaces between neighboring-in contact anatase particles[218]. Therefore, for a finer anatase nanoparticle system, less energy will be required to transform into rutile than for a coarser anatase system.

The strong self-assembling aggregating behavior of $\mathrm{TiO}_{2}$ particles is observed from the SEM and optical imaging. Aggregation of the $\mathrm{TiO}_{2}$ particles is necessary to promote continuous structures; however, controlling aggregation in colloidal systems is a key aspect for the inks' deposition. The use of polymers and co-solvents is a common way for controlling the viscosity and dispersion properties of such systems. In the case of the co-solvents, the different vapor pressure exhibited by these enables the tuning of the drying characteristics of the films/patterns; thus reducing the macroscopic defects in the deposited material[84]. 

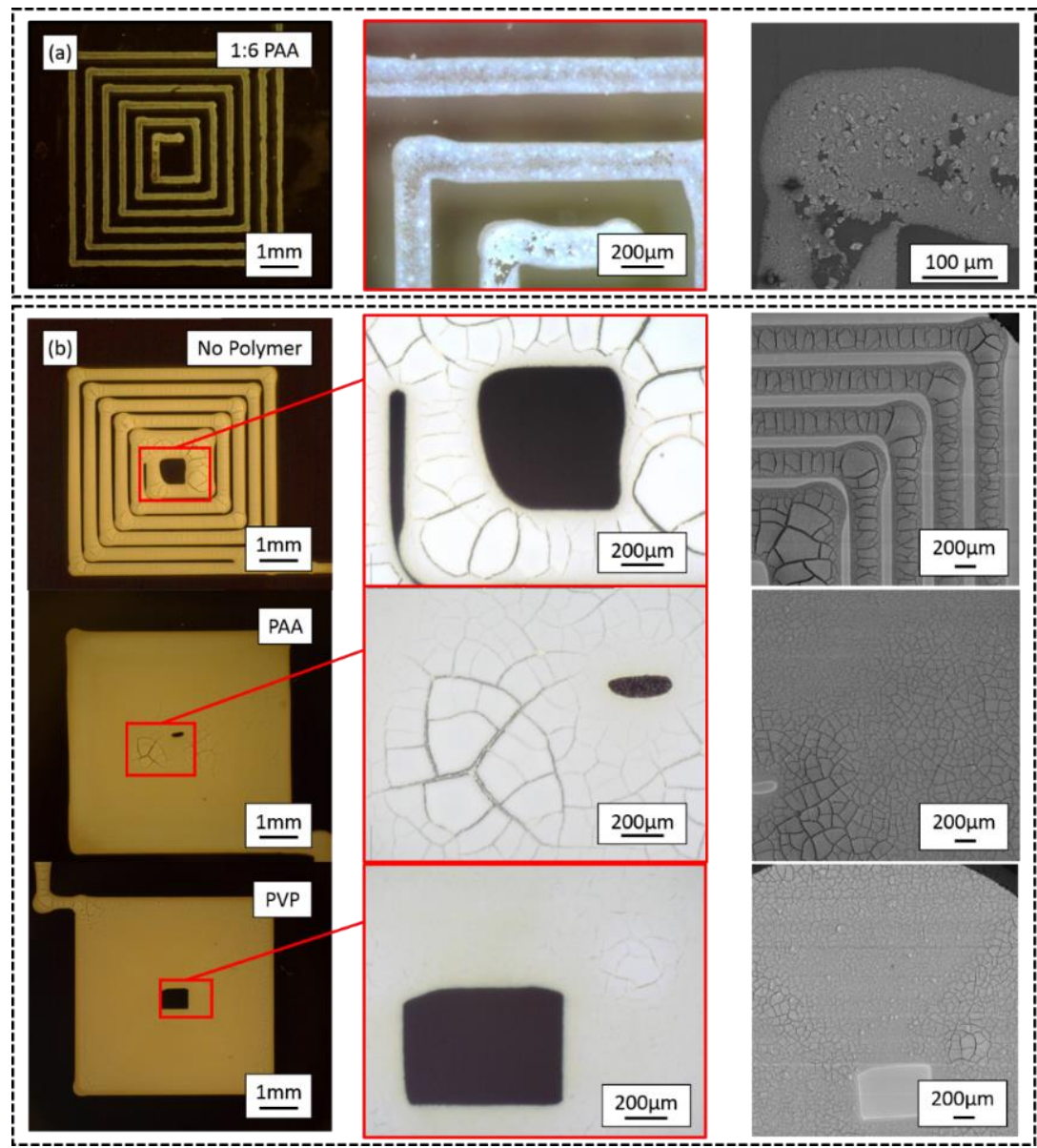

Figure 38. Optical and SEM images of the directly written TALH:TiO 2 films and patterns from different polymer formulated (a) 1:6 PAA and (b) (1:12) inks on Ag-PEN substrates. With CDW speed of $5 \mathrm{~mm} / \mathrm{s}$ and pressure of $20 \mathrm{KPa}$ and $150 \mu \mathrm{m}$ inner diameter nozzles.

The addition of polymers (PAA or PVP) reduces the cracking the $\mathrm{TiO}_{2}$ films / patterned. $\mathrm{TiO}_{2}$ discontinuous regions can be observed for the pattern made from (1:6) PAA ink. In contrast, for the 1:12 formulations, full coverage of the patterned regions is observed; however, crack formation results from the strong aggregation of the $\mathrm{TiO}_{2}$ particles when the solvent evaporates. Close examination of the surface and selfassembly of the $\mathrm{TiO}_{2}$ particles in the uniform regions shows similar aggregation behavior regardless of the formulation (1:6 or 1:12, with/without polymers) or the deposition technique used, DB or CDW, see Figure 34 .

Transmission electron microscope (TEM) images and selected area electron diffraction (SAED) patterns of representative samples were taken to further investigate the $\mathrm{TiO}_{2}$ formations from TALH after different treatments. The as received $\mathrm{TiO}_{2}$ particles can be observed in Figure 39 (a), overlapping of the aggregated particles is observed, as well as their single crystalline nature. Regarding the samples prepared from $\mathrm{TALH}: \mathrm{TiO}_{2}$ films, Figure 39(c) and (d), additional crystalline formations are noted. For the sample from $\mathrm{TAHL}^{\mathrm{TiO}}{ }_{2}$ (1:6) PAA 6hUV, the high resolution TEM image shows a meniscus-like formation with well defined lattice fringes, characteristic of materials with crystalline structures; for the TALH:TiO 2 (1:6) $150^{\circ} \mathrm{C}-30$ min treated film, Figure 39 (c), a polycrystalline structure is observed between two $\mathrm{TiO}_{2}$ particles. SAED patterns confirm the polycrystalline character of such formation to be anatase phase. 

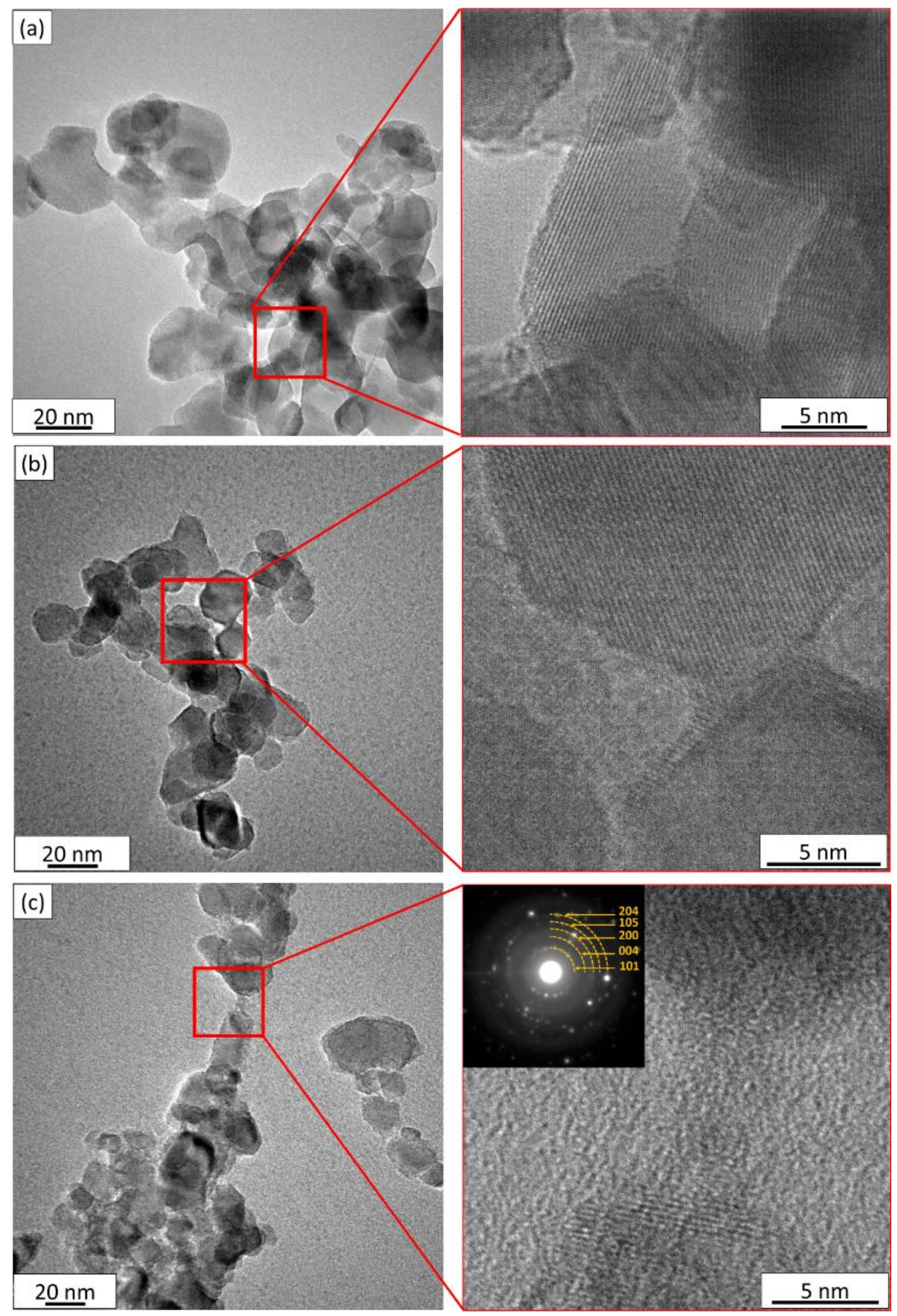

Figure 39. TEM images of the (a) as received $\mathrm{TiO}_{2}$ Aeroxide particles, (b) TALH:TiO 2 (1:6) - PAA film after 4h UV exposure, and (c) TALH:TiO 2 (1:6) film after $150^{\circ} \mathrm{C}-30$ min treatment. HR-TEM images of the enclosed regions show the lattice fringes of the crystals and the bridging formations from TAHL in (b) and (c). Diffraction pattern taken for the enclosed region in (c). 
XPS shows a systematic decrease in the O:Ti ratio towards the stoichiometric value 2, as the UV energy and $\mathrm{TiO}_{2}$ initial content are increased; this ratio, greater than 2 for all the fabricated films, also exhibits a dependence on the type of energy treatment employed for curing of the film when polymers are incorporated. For PAA, the O:Ti ratio values are closer to 2 when utilizing UV energy. On the other hand, for the PVP this value was closer to 2 when using $150^{\circ} \mathrm{C}-30 \mathrm{~min}$ as energy treatment. The differences in these polymer structures may be associated to such trends; however further studies about the fundamental reasons behind such stoichiometric variation may provide further insight.
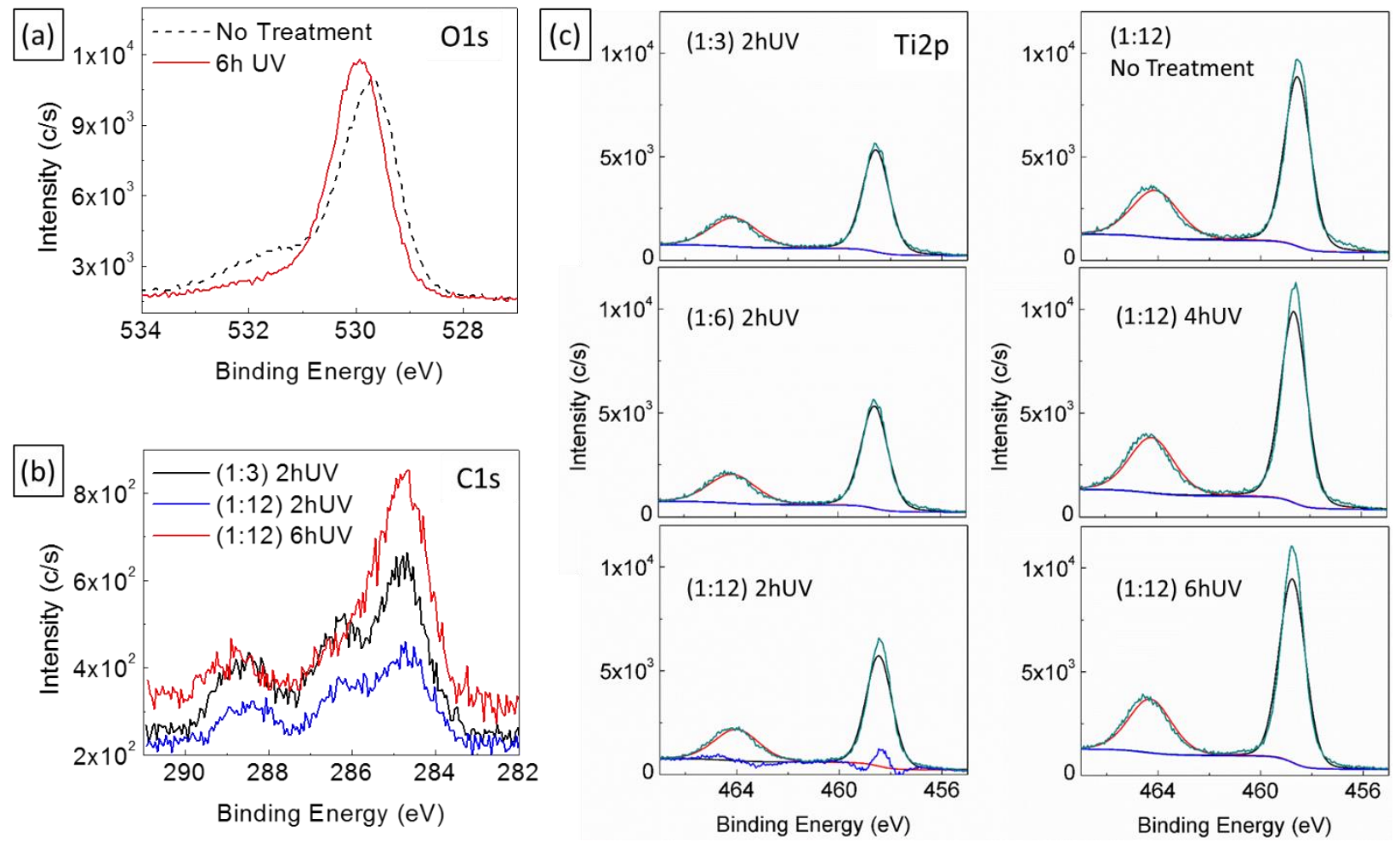

Figure 40. X-ray photoelectron spectroscopy of the films exposed to different UV conditions (a) O1s detailed scan for (1:12) no-polymer films; (b) C1s and (c) Ti2p detailed scans. The cyan line in (c) corresponds to the experimental XPS data.

Detailed scan for the 01s peak shows a shoulder feature at $\sim 531.5 \mathrm{eV}$, characteristic of a hydrated state of $\mathrm{TiO}_{2}$, which diminishes as UV exposure is increased. Similar decrease of this oxygen energetic state has been reported for $\mathrm{TiO}_{2}\left(\mathrm{H}_{2} \mathrm{O}\right)$ upon thermal annealing[57].The Ti2p peak, is observed to have a fixed position for all no-polymer cases, with the characteristic $\Delta \mathrm{E}_{\mathrm{b}}$ of $\sim 5.75 \mathrm{eV}$. However, the intensity ratio between the Ti2p spin-orbit splitting peaks Ti2 $p_{1 / 2}$ at $464.5 \mathrm{eV}$ and Ti2 $\mathrm{p}_{3 / 2}$ at $458.75 \mathrm{eV}$ deviates from the theoretical 1:2 area ratio as the initial $\mathrm{TiO}_{2}$ crystalline phase and UV exposure time are increased (Figure 40 (c)) This result is attributed to the increased energy state induced by the photocatalytic nature of the $\mathrm{TiO}_{2}$ system upon UV exposure. For the polymer added systems, a slight shifting (of $\sim 0.5 \mathrm{eV}$ ) of the Ti2p peak towards lower binding energy values is observed (Figure 41). Interestingly, the intensity ratio between the Ti2p spin-orbit splitting peaks Ti2 $p_{1 / 2}$ and Ti2 $p_{3 / 2}$ is very close to the theoretical 1:2 area ratio for the PAA formulation, regardless of the energy treatment employed, and in contrast to the deviation trend displayed for all the other formulations. 

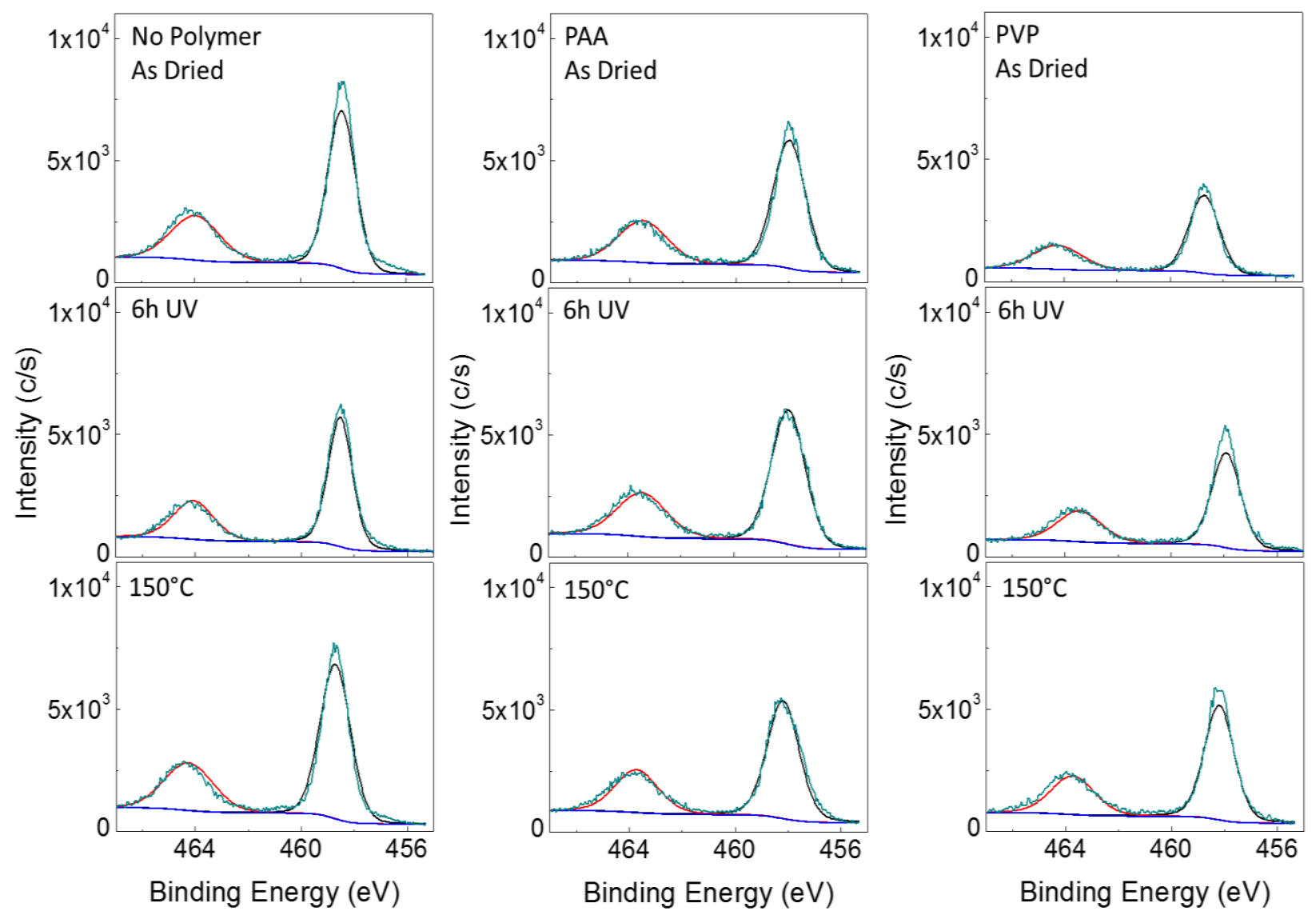

Figure 41. Detailed scan of Ti2p peak for the 1:12 TALH:TiO 2 films from No polymer, PAA and PVP formulations. The cyan line indicates corresponds to the raw XPS data.

\subsection{Photocatalytic Properties}

The photocatalytic performance of the films from lower initial crystalline $\mathrm{TiO}_{2}$ content inks is observed to be more pronounced than for the higher concentration (1:12) counterparts (in turn influenced by the incorporation of polymer additives), as indicated by the apparent first-order degradation constant $\left(k_{\text {app }}\right)$ values, see Table 13 . The addition of polymers to the ink formulations has an increasing effect on the $k_{a p p}$ values for the films with low initial particle concentration (1:6). Particularly, for films from PAA formulations, an important increment of the $k_{a p p}$ (nearly two times) is observed for the (1:12) films. Remarkably, the PAA films showed no visible deterioration after the photocatalysis experiments, in contrast to the PVP film which were almost completely fragmented and delaminated from the substrates. Damage of the 1:6 films was more evident than that of 1:12 films after photocatalytic degradation of MB. The photocatalytic performance also shows a strong dependence on the energy treatment used for the films synthesis; generally, the UV treatment led to better photocatalytic performance than the mild heat treatment (see Figure 42). The films' photocatalytic performance is comparable to similar $\mathrm{TiO}_{2}$ mesostructured films[38,220].

AFM results, indicate higher roughness values (from AFM measurements) for the best photocatalytically performing films. This characteristic behavior, related to the $\mathrm{TiO}_{2}$ crystallite size, favors the degradation 
of MB on films with larger amount of nano-structured features. An opposite trend is observed between the roughness values from profilometry and AFM, this difference is attributed to the mesoscopic structure of the films and the different techniques' resolution. The AFM scans, probed areas of $1 \mu \mathrm{m} \times 1 \mu \mathrm{m}$, detecting surface details in the nano-scale (in the crystallites formed from TALH range); whereas the profilometry measurements provide information from larger structures (in the micron-scale), such as the aggregates formed by the initially added $\mathrm{TiO}_{2}$ nanoparticles at the ink formulation stage.

Table 13. Apparent first-order degradation rate constant $k_{a p p}$ and time for $50 \% \mathrm{MB}$ degradation from different $\mathrm{TiO}_{2}$ films. AFM and Profilometry roughness measurements for the films.

\begin{tabular}{|c|c|c|c|c|}
\hline \multirow[b]{2}{*}{ Sample } & \multirow[b]{2}{*}{$\begin{array}{c}k_{a p p} \times 10^{-2} \\
\left(\min ^{-1}\right)\end{array}$} & \multirow[b]{2}{*}{$\begin{array}{c}\text { Time for } \\
\text { Degradation > } \\
50 \%(\min )\end{array}$} & \multicolumn{2}{|c|}{ Roughness } \\
\hline & & & $\begin{array}{l}\text { AFM }(\mathrm{nm}) \\
(2-5 \mathrm{~nm} \text { tip } \\
\text { radius) }\end{array}$ & $\begin{array}{l}\text { Profilometry }(\mu \mathrm{m}) \\
(2 \mu \mathrm{m} \text { tip radius) }\end{array}$ \\
\hline Blank & 0.11 & * & --- & --- \\
\hline 1:6 6hUV & 0.80 & 90 & 2.90 & 3.87 \\
\hline $1: 6150^{\circ} \mathrm{C}$ & 0.62 & 105 & 1.97 & 5.01 \\
\hline 1:12 6hUV & 0.52 & 120 & 1.53 & 3.46 \\
\hline $1: 12150^{\circ} \mathrm{C}$ & 0.39 & --- & 0.98 & 2.47 \\
\hline PAA 1:6 6hUV & 1.19 & 75 & 12.27 & 1.73 \\
\hline PAA 1:6 $150^{\circ} \mathrm{C}$ & 0.73 & 105 & 17.42 & 1.82 \\
\hline PAA 1:12 6hUV & 1.12 & 75 & 11.63 & 1.43 \\
\hline PAA $1: 12150^{\circ} \mathrm{C}$ & 0.60 & 150 & --- & 12.66 \\
\hline PVP 1:6 6hUV & 1.55 & 45 & 11.71 & 2.49 \\
\hline PVP $1: 6150^{\circ} \mathrm{C}$ & 0.89 & 75 & 10.22 & 6.61 \\
\hline PVP 1:12 6hUV & 0.56 & 120 & 8.18 & 2.47 \\
\hline PVP 1:12 $150^{\circ} \mathrm{C}$ & 0.37 & --- & 9.53 & 3.53 \\
\hline
\end{tabular}

* The maximum degradation for the Blank (control) solution was $12 \%$ after 150 min UV exposure. 

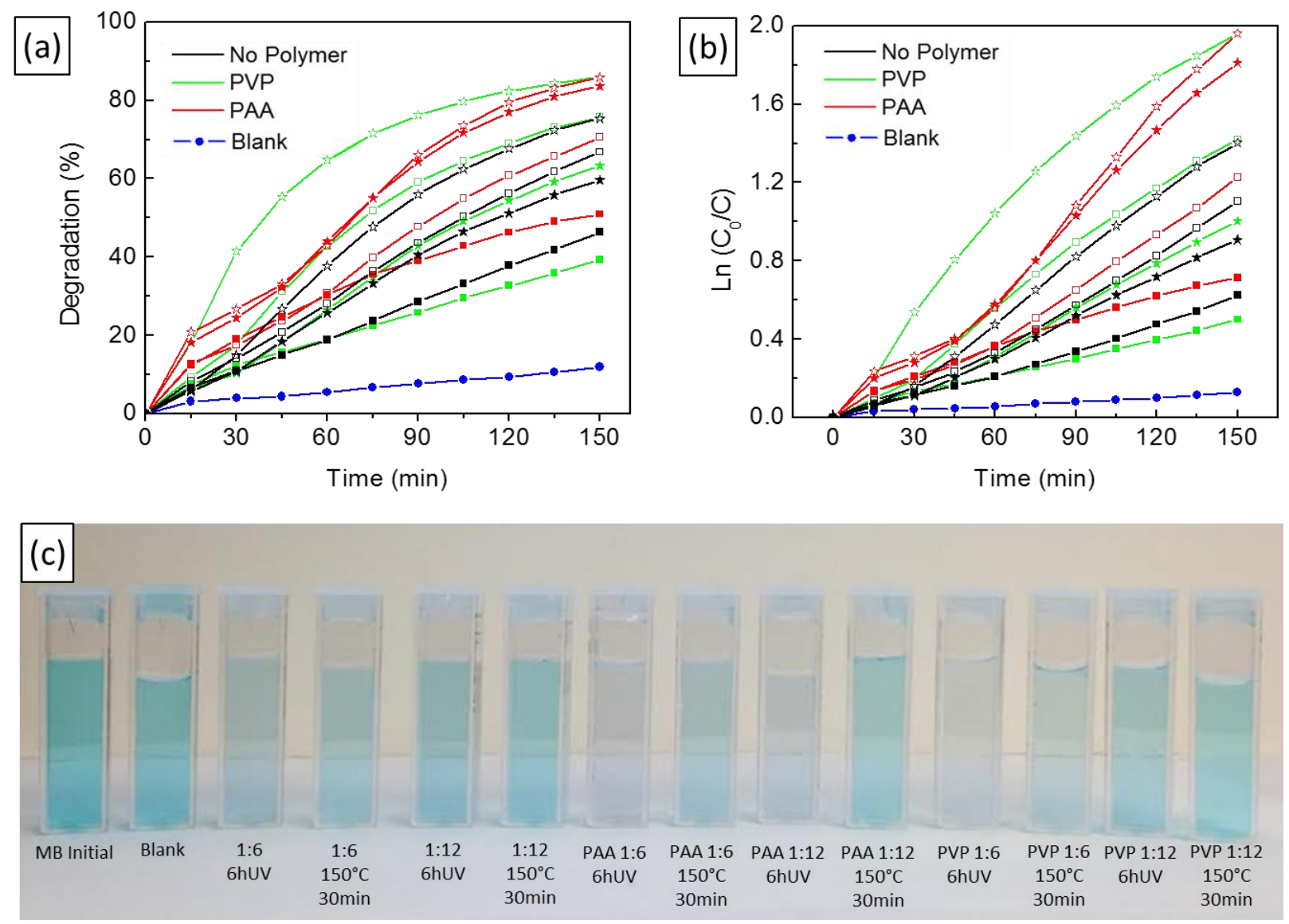

Figure 42. (a) Methylene blue (MB) degradation (\%) and (b) linearized $M B$ concentration change in time, with different $\mathrm{TALH}: \mathrm{TiO}_{2}$ films. Open and filled symbols represent (1:6) and (1:12) TALH:TiO $\mathrm{T}_{2}$ formulations respectively. Star symbols represent $6 \mathrm{~h}$ UV treatment and square symbols represent $150{ }^{\circ} \mathrm{C}-30$ min annealing. (c) Image of the $M B$ initial and aliquots of the decomposed $M B$ solutions after 150 min UV irradiation.

\subsection{Ink Precursors' Chemistry Influence}

In the $\mathrm{TiO}_{2}-\mathrm{TAHL}$ system, the different ink constituent chemistries have been identified to affect the resulting materials' (as films or patterns) crystallinity, microstructure, mechanical stability and photocatalytic activity.

When depositing the material onto the substrates, the acidity or basicity of the ink and the substrate dictates the compatibility of these two, allowing for the adsorption of the inks' anchoring groups onto the substrate surface. In the $\mathrm{TiO}_{2}-\mathrm{TALH}$ system, the primary groups available for such interactions are carbonyl and hydroxyl groups. The presence of ammonia ions stabilizes such groups making TALH almost non-reactive in neutral solutions. When $\mathrm{TiO}_{2}$ and $\mathrm{TALH}$ are mixed together, the TALH is adsorbed onto the $\mathrm{TiO}_{2}$ surface through the available Ti atoms on the particles' surface and the hydroxyl groups from TALH, in a similarly way to that of carboxylic groups attaching to rutile[221]. The high compatibility of these two components is indicated by their behavior in solution, where the homogeneity of the dispersions is evident and no further phase separation is observed (other than the initial solid/liquid differentiation system). 


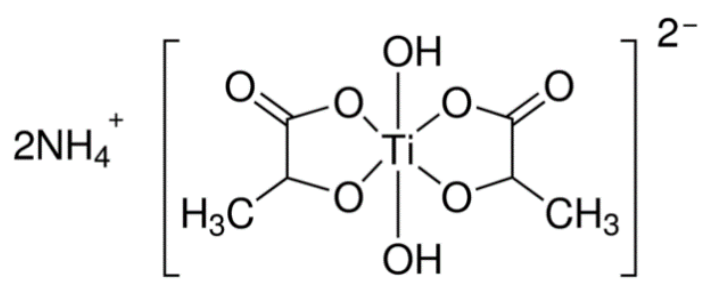

Figure 43. Chemical structure of the Titanium(IV) bis(ammonium lactato) dihydroxide (TALH) compound.

As more constituents are added to the system, different reactions may occur. A one-to-one relation between the acidic $\mathrm{H}+$ or basic $\mathrm{OH}$ - groups and the ammonium lactate ions present in the TALH molecule has been reported to be necessary to neutralize the ammonium lactate and allow the $\mathrm{TiO}_{2}$ nucleation at low temperatures[57]. However, from the current investigation of the TALH system, such acid/base conditions are far too strong and may lead to the substrate's (ITO) deterioration once it comes to contact with such a strong ink. The polymeric agents (added to tailor the inks rheological properties), having different polymer chemistries, also participate in the $\mathrm{TiO}_{2}-\mathrm{TALH}$ interactions, and moreover in the ink $\left(\mathrm{TiO}_{2}+\mathrm{TALH}\right)$ interactions with the substrates. The contact angle measurements indicate that the relative surface energy between the substrate and the ink changes as the different polymers are incorporated to the system. Diethylene glycol (DEG) was also incorporated to the $\mathrm{TiO}_{2}-\mathrm{TALH}$ formulations (at the preliminary stages of the ink formulation) to explore its effect on the adhesion to the ITO substrates; however instead of improving the ink's wetting onto the substrates it made the formulations to "bulge up" not retaining the film or pattern shape when applying the materials through DB or CDW, respectively. From the contact angle measurements of water on the ITO/PET substrates, a rather hydrophobic character of the ITO coating is observed (with a contact angle of $\sim 106^{\circ}$ ). Nevertheless, it has been reported that the surface of ITO contains mainly $\mathrm{OH}$ groups[222-225]; therefore, the acidic inks are more likely to be adsorbed than the more neutral counterparts.

The PVP side-groups available for interaction with $\mathrm{TALH}$ and / or $\mathrm{TiO}_{2}$, are carbonyl type; whereas PAA has carboxylic groups, which with the extra $\mathrm{OH}$ ends, promotes stronger binding to the $\mathrm{TiO}_{2}$ surface. Also, as the inks are brought in contact to the substrates, their bonding is again facilitated through the carboxylic groups. A final remark about this is that the substrate's $\mathrm{pH}$ should necessarily be considered in relation to the ink's $\mathrm{pH}$ for the design of the ink-substrate system. Because of the carboxylic groups' tendency to attach to the $\mathrm{Ti}$ atoms in $\mathrm{TiO}_{2}$, it is proposed that the PAA chains serve as scaffold structures for the $\mathrm{TiO}_{2}$ crystallites, forming from $\mathrm{TALH}$, to form bridging structures that connect the initially added $\mathrm{TiO}_{2}$ particles. From the photocatalysis perspective, it is found that the films made from inks with polymeric agents resulted in more efficient photocatalytic decomposition of organic dyes (MB) than when using no-polymer $\mathrm{TiO}_{2}$-TALH films mediated photocatalysis. Moreover, from the samples used for the photocatalysis experiments, it was observed that, the deterioration of the PVP films was much greater than that of the PAA films.

Additionally, through the assessment of the different inks' chemistries and various substrate material interactions, it is observed that they not only influence the materials final properties but also their printing by preventing or promoting clogging of the nozzles depending on their material. Generally, the more dissimilar the $\mathrm{pK}_{\alpha}$ values for the inks and the nozzle materials, the higher tendency of having clogging issues when printing due to the better wetting of the inks onto the nozzle surfaces. 


\subsection{Electrical Properties}

The electrical resistivity of the synthesized films from the no-polymer and PAA 1:12 formulations were investigated by means of the IV characteristics. No significant variation was observed on the IV curves, when probing on the metal contacts and directly on the $\mathrm{TiO}_{2}$ surface. The thickness of the sputtered $\mathrm{Ag}$ is $\sim 100 \mathrm{~nm}$; the topography of the film exhibits high roughness ( $5 \mu \mathrm{m}$ as measured using profilometry). Therefore, the resistivity calculations are done considering the probe area, calculated from the distorted area of the samples from plan-view images to be $\sim 0.008 \mathrm{~mm}^{2}$. The sputtered Ag-contacts' area is $\sim 0.9$ $\mathrm{mm}^{2}$.
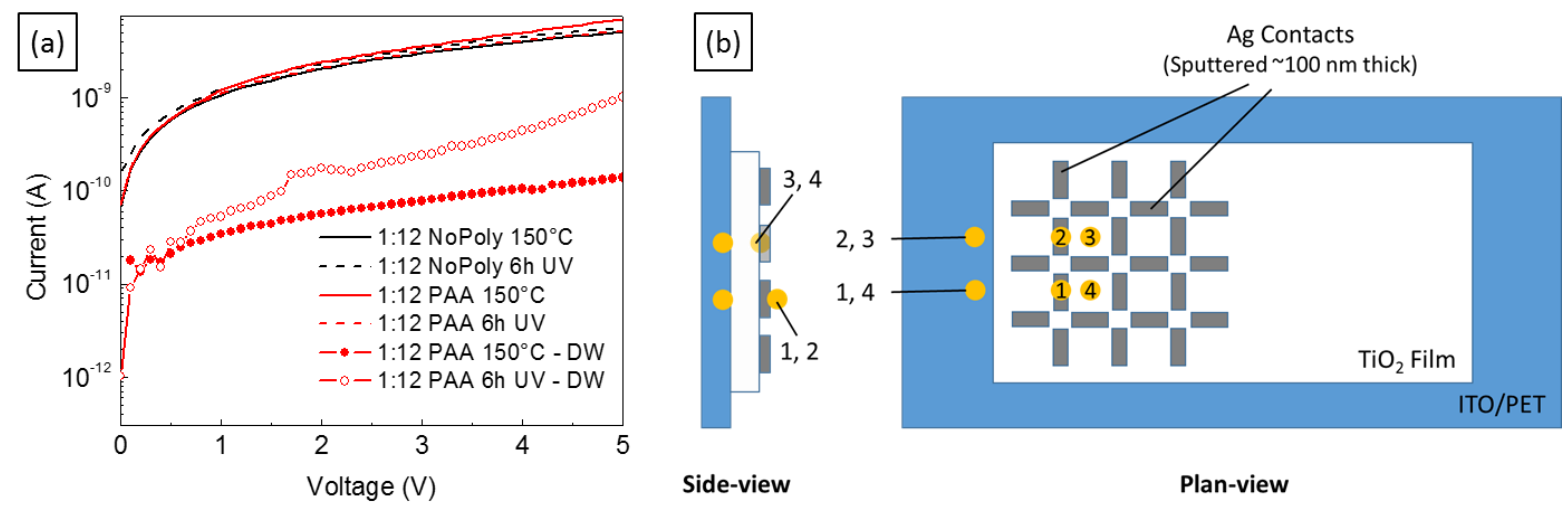

Figure 44. IV Characteristics of the no-polymer and PAA TALH:TiO 2 films treated under $150^{\circ} \mathrm{C}-30 \mathrm{~min}$ and $6 \mathrm{~h}$ UV light $(\lambda=254 \mathrm{~nm})$; and (b) schematic of the samples and the probing sites used for IV characterization.

The calculated resistivity values for the different films (summarized in Table 14), are comparable to those obtained for $\mathrm{TiO}_{2}$ polycrystalline thin films $[76,77,226]$. Generally, the electrical properties of the $\mathrm{TiO}_{2}$ polycrystalline films depend on the films microstructure and purity of the $\mathrm{TiO}_{2}$ phases. The investigated films contain relatively high quality $\mathrm{TiO}_{2}$ nanoparticles (Aeroxide ${ }^{\circledR}$, purity $>99.5 \%$ ) and a mixture of organic compounds (TALH and PAA) which may be responsible for the variation of the experimental resistivity values. It is also observed that the calculated resistivity values for the films fabricated using CDW are one order of magnitude higher than the DB counterparts. The former samples exhibiting larger thickness and roughness values. Further investigation of the electrical characteristics of titania films prepared from these formulations, and their dependence on the deposition technique is proposed as future work.

Table 14. Film thickness measured by profilometry and electrical resistivity of the films as calculated from the IV characteristics.

\begin{tabular}{|c|c|c|c|}
\hline Film & Thickness ( $\mu \mathrm{m})$ & Roughness ( $\mu \mathrm{m})$ & Resistivity $(\Omega \mathrm{cm})$ \\
\hline 1:12 NoPoly $150^{\circ} \mathrm{C} 30 \mathrm{~min}$ - DB & 8.093 & 2.495 & $9.50 \times 10^{7}$ \\
\hline 1:12 NoPoly 6hUV - DB & 12.113 & 2.122 & $5.72 \times 10^{7}$ \\
\hline 1:12 PAA $150^{\circ} \mathrm{C} 30 \mathrm{~min}-\mathrm{DB}$ & 12.01 & 3.122 & $6.01 \times 10^{7}$ \\
\hline 1:12 PAA 6hUV - DB & 21.429 & 3.964 & $3.43 \times 10^{7}$ \\
\hline 1:12 PAA $150^{\circ} \mathrm{C} 30 \mathrm{~min}$ - DW & 37.249 & 9.156 & $8.07 \times 10^{8}$ \\
\hline 1:12 PAA 6hUV - DW & 38.919 & 8.747 & $2.45 \times 10^{8}$ \\
\hline
\end{tabular}




\subsection{Concluding Remarks}

The investigated hybrid Ti-organic/ $/ \mathrm{TiO}_{2}$ ink systems, exhibit great potential for their use in additive manufacturing using CDW. The viscosity and printing properties of the $\mathrm{TiO}_{2}-\mathrm{TALH}$ inks are shown to be tailored through solvent amount and polymer addition and $\mathrm{TALH}: \mathrm{TiO}_{2}$ ratio and meet the requirements of specific printing techniques beyond direct writing. The mild temperature and UV irradiation treatments used to transform the amorphous/crystalline formulations to semi-crystalline/crystalline films allow the application of these materials on flexible substrates. From the energy treatments explored, it is observed that the upper limit for crystallite size is comparable to the initial $\mathrm{TiO}_{2}$ particle size while smaller particles nucleate from TALH. The crystalline $\mathrm{TiO}_{2}$ initial concentration in the system is used as (a) a provider of $\mathrm{TiO}_{2}$ nucleation and photocatalytic active sites for organics decomposition; and (b) a control parameter for ink rheology and solvent evaporation. The use of polymers as stabilizing and ink thickening agents, is also found to affect the formation of $\mathrm{TiO}_{2}$ crystallites from $\mathrm{TALH}$. In the system, the energy supplied via the energy treatments is necessarily used for polymer decomposition as well as for the $\mathrm{TiO}_{2}$ nucleation from TALH. Moreover, the different polymer chemistries greatly influence the ink/substrate interactions, and affect the crystallite formation by partial reaction with the ammonium lactate groups present in TALH. Finally, the presence of carboxylic groups in the polymer additives may favor the bridging of neighboring $\mathrm{TiO}_{2}$ particles.

The microstructure of the direct written and doctor bladed films are observed to be porous and show negligible variation depending on the deposition technique; the assembling of the colloidal system upon drying and curing is dominated by strong $\mathrm{TiO}_{2}$ particle aggregation forces. The microstructures obtained are suitable for applications where high surface area is important. Relationships between the different robotic printing parameters and obtained macro/micro-structures were established. Additionally, the electrical resistivity of the polycrystalline $\mathrm{TiO}_{2}$ films is in the order of $10^{7}$ to $10^{8} \Omega^{*} \mathrm{~cm}$, and comparable to that of $\mathrm{TiO}_{2}$ films obtained from high-energy intensive conditions reported in literature. Further work on direct writing of patterns from the proposed ink system, and the effect of the curing (heat/UV exposure) treatments with respect to the patterns' final dimensions, residual stress[227], and electrical properties will provide additional means for the implementation of these ink systems in flexible electronics.

Photocatalysis degradation of methylene blue highlights the potential of the investigated material system for organic chemicals decomposition. The TALH:TiO 2 ratio and the type of energy treatment employed for the film synthesis, i.e. UV exposure or mild annealing, are found to have great influence on the photocatalytic degradation rate, which is concomitantly related to the roughness of the films and their mesoporosity. 
Chapter 6: Multiphase Materials Systems I: Microstructuring and Enabling 3D Printing of $\mathrm{TiO}_{2}$ Foams

3-D printing of hierarchically ordered cellular materials with tunable microstructures is a major challenge from both synthesis and scalable manufacturing perspectives. A simple, environment-friendly, and scalable concept to realize morphologically and microstructurally engineered cellular ceramics is herein proposed and realized, by combining direct foam writing with colloidal processing. These cellular structures are widely applicable across multiple technological fields including energy harvesting, waste management / water purification, and biomedical. $\mathrm{TiO}_{2}$ is used as a metal-oxide model system, having important applications due to its interesting semiconducting properties, tunable band gap, photocatalytic properties, bio-compatibility and abundance.

The concept marries sacrificial templating with direct foaming to synthesize multi-scale porous $\mathrm{TiO}_{2}$ foams that can be 3D printed into planar, free-standing, and spanning hierarchical structures. The latter being reported for the first time. This approach represents clear progress in the fabrication of $\mathrm{TiO}_{2}$ foams, traditionally fabricated using laborious multi-step methods[157,162,169-171]. Special emphasis is placed in investigating the relationships between the foams' composition, processing, microstructure, surface area properties, and photocatalytic performance. The proposed synthesis and scalable manufacturing method can be extended to fabricate similar structures from alternative ceramic foam systems, where control of the porosity and surface properties is crucial, demonstrating the great potential of this synthesis approach.

\subsection{TAHL:TiO 2 Foam - Design}

The TALH Foam Ink is a multiple-phase system consisting of solid, liquid and gaseous phases, as a hybrid synergistic approach that combines the direct foaming and sacrificial template mechanisms, to produce porous structures with features in the micro-, meso- and macro pore range, using environment - friendly and abundant material precursors. The emulsion is designed to be an oil-in-water system, in which the aqueous phase is a $\mathrm{TiO}_{2}$ inorganic (solid) / Ti-organic complex colloidal suspension, and the oil phase serves as dispersion and stabilizing agent[228,229] for the gas-liquid mixture. The gas phase consists of air bubbles that are trapped in the liquid matrix. See Figure 45 . Once the foam-ink is prepared, it can be shaped into the desired dimensions using continuous-flow direct writing and allowed to dry. Next, upon heat post-processing treatments, the TALH (in contact with the $\mathrm{TiO}_{2}$ primary particles) is transformed into $\mathrm{TiO}_{2}$ aiding the bridging between neighboring particles $[60,128]$ and providing mechanical and chemical stability to the structures. Such foam walls exhibit additional levels of porosity realized from the primary particle aggregation, and the removal of the oil phase and other organic additives used for the ink formulation. Also, for this foam system, additional stabilizing effects can be attributed to the $\mathrm{TiO}_{2}$ particles, aiding to maintain the trapped air bubbles, a characteristic of multiple emulsion systems.[230,231] 


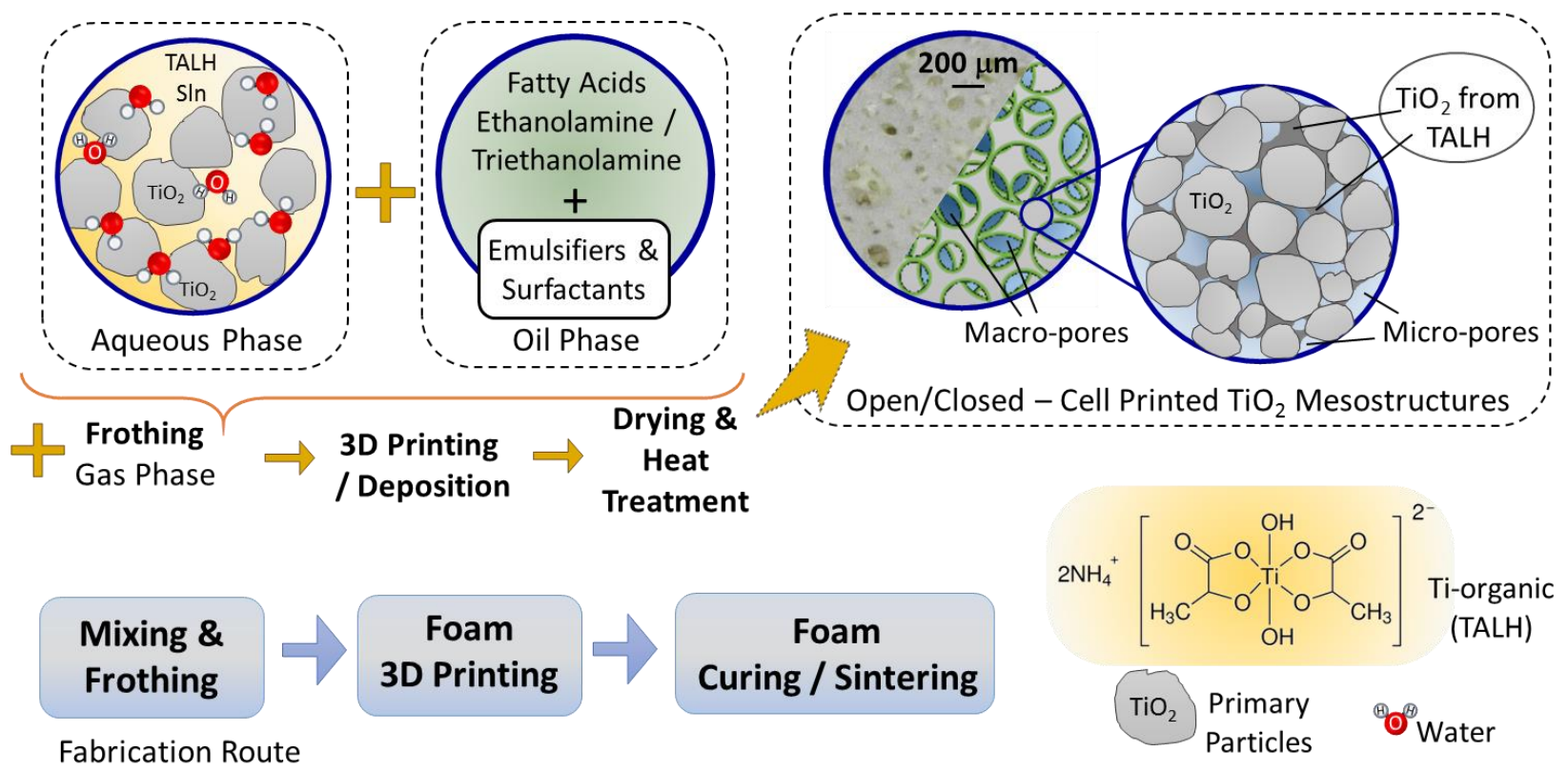

Fabrication Route

Figure 45. Schematic synthesis route, and microstructure (photograph), of mesoporous TiO foams. $^{2}$

Being a multi-phase material system, there are significant interactions between the different foam constituents, resulting in complex relationships affecting the foams' morphology, microstructure, rheological properties, and surface and porosity properties to name a few. Thus, the foams' morphologies are tailored by varying their composition and frothing conditions, thus affecting their viscosity and their printing parameters and space, i.e. planar vs. 3D structures, as show in this chapter.

\subsection{Sustainability Considerations}

This synthesis approach, aims for the development of sustainable and relatively simple synthesis methods and formulations, to produce hierarchically ordered mesoporous cellular ceramics with tunable cell configurations (i.e. closed-/open-cell ceramic foams) and surface area properties. In particular this investigation addresses 8 of the 12 principles of green chemistry[174], for the developed materials and synthesis approach. Waste prevention is accomplished by incorporating additive manufacturing (i.e. 3D printing), since all the prepared foam batches can be printed in the exact amounts, geometries and substrate locations. The atom economy $(A E)$ is estimated from the foam-ink design calculations and thermogravimetric analysis (TGA). The synthesis method and resulting materials implement the least hazardous chemical synthesis principle, by involving non-toxic, renewable and bio-compatible ink precursors. The oil phase of the foams consists of fatty acids compounds commonly used in the cosmetic industry [232]. Similarly, the use of ethanolamine and triethanolamine as emulsifiers is kept to minimum amounts, also comparable to those encountered in cosmetic products [233]. In addition, the utilization of TALH as Ti-organic precursor, allows the formulation of aqueous based systems, exhibiting very slow hydrolyzation rates in neutral pH conditions [57]; hence avoiding the need to use organic solvents, and allowing ample time for their printing in ambient conditions. These conceptual considerations, make this ink system inherently safe and therefore transferable to industry. Finally, the synthesized foams can be recycled/regenerated[234]; and are generally safer than the primary $\mathrm{TiO}_{2}$ nanoparticles for applications such as water purification[235], being larger in size for easier recovery in case of accidental release to the environment. 


\subsection{Foam Rheology and Microstructure}

Different mixtures of the foam were prepared by varying the amount of aqueous solution (water+TALH), $\mathrm{TiO}_{2}$ primary particles, and oil phase, see Table 15. It is observed that the incorporation of gas phase was achieved in various extents depending on their composition, i.e. the different water/oil ratios resulted in foams with a varying degree of stability (retention of the gas bubbles and/or avoidance of their coalescence). This can be partially assessed through viscosity measurements at different time intervals after the initial frothing, and through image analysis of the wet-foams' microstructural evolution, see Figure 46. Typically, the gas bubbles will coalesce and grow over time (as indicated with arrows for the L75-S5.5-019.5 MEA foam after 4 days). Also, the foam volume will decrease as indicated by dashed ovals from Figure 46 (a) and (b), and some drainage will also be characteristic of foam relaxation - see dashed ovals in Figure 46 (c) and (d).
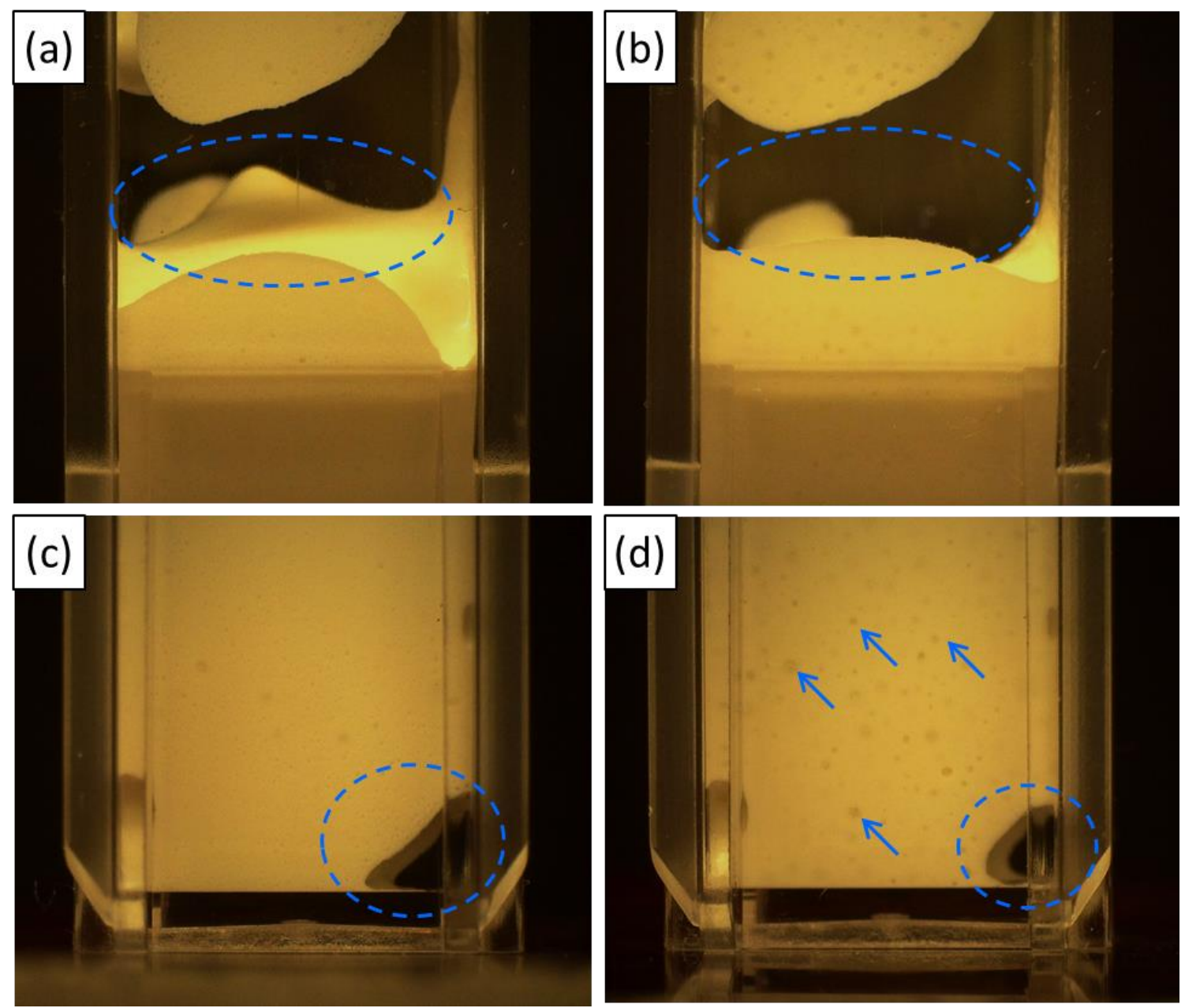

Figure 46. Optical images of the 1:12 TALH:TiO 2 L75-S5.5-019.5 MEA foam inside a polystyrene cuvette for stability assessment. (a) top and (c) bottom sections as placed in the cuvette, and (b) top and (d) bottom sections after 4 days. The cuvette width is of $10 \mathrm{~mm}$. 
Table 15. Phase amounts in the different $\mathrm{TiO}_{2}$ Foams.

\begin{tabular}{lcccc}
\hline \multicolumn{1}{c}{ Foam Ink Name } & Liquid & $\mathrm{TiO}_{2}$ Solids (vol\%) & Oil (vol\%) & Aqueous-SIn:Oil \\
\hline L75-S3-022 & 75 & 3 & 22 & 3.42 \\
L75-S5.5-019.5 & 75 & 5.5 & 19.5 & 3.86 \\
L82-S4-014 & 82 & 4 & 14 & 5.87 \\
L86-S3-011 & 86 & 3 & 11 & 7.80 \\
\hline
\end{tabular}

The microstructure of the prepared foams, is also visible affected by the choice of liquid-solid-oil amounts, and can be tailored to exhibit open- or closed-cell structures with varying macro-pore sizes, as is discussed later in this chapter. As an example, the macropores obtained for foam L75-S3-022 are much smaller roughly 39\% - than those obtained for foam L86-S3-011 (with half the amount of oil phase). Also, the thickness of the walls is observed to decrease as the oil phase is increased, see Figure 47.

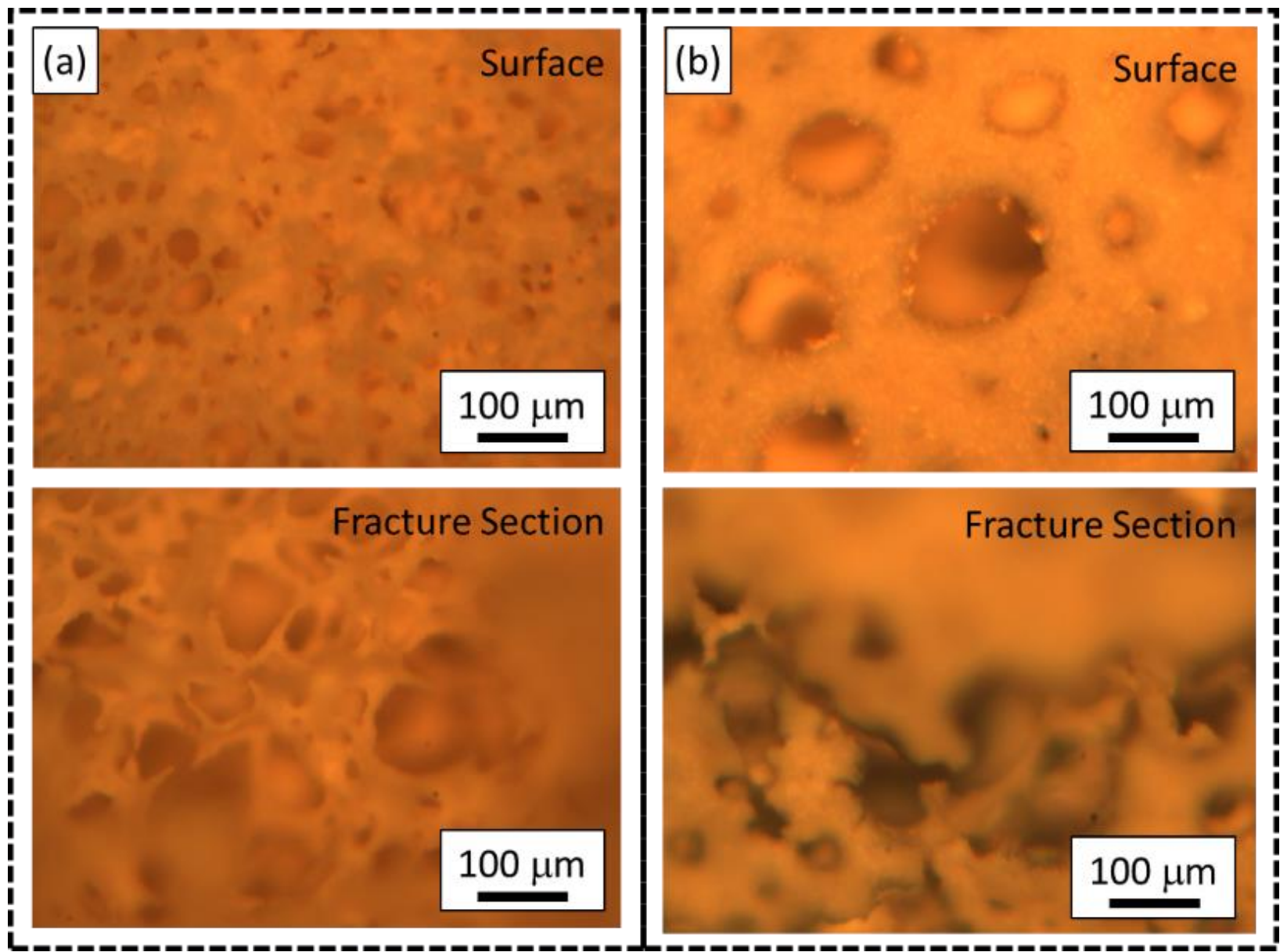

Figure 47. Optical microscope images of the foams surface and inner sections obtained from formulations (a) L75-S3022 and (b) L86-S3-O11, and treated at $150^{\circ} \mathrm{C}-30 \mathrm{~min}$.

Accordingly, the viscosity displays variation depending on the water/oil ratio, exhibiting higher values for lower water/oil ratios, see Figure 48 (a). The stabilization role of the oil phase, is attributed to the reduction of the gas-slurry surface tension and to the modification of the foams' viscoelastic properties[236], i.e. it provides the emulsion with yield-stress fluid properties, highly desirable for CDW. 
The gas retention (foam stability) is also visible from the viscosity measurement at different points in time after the foams preparation. From Figure 48 (b), a decrease in the viscosity is observed after 1 day of prepared; re-frothing of the foams can be used to partially restore it.
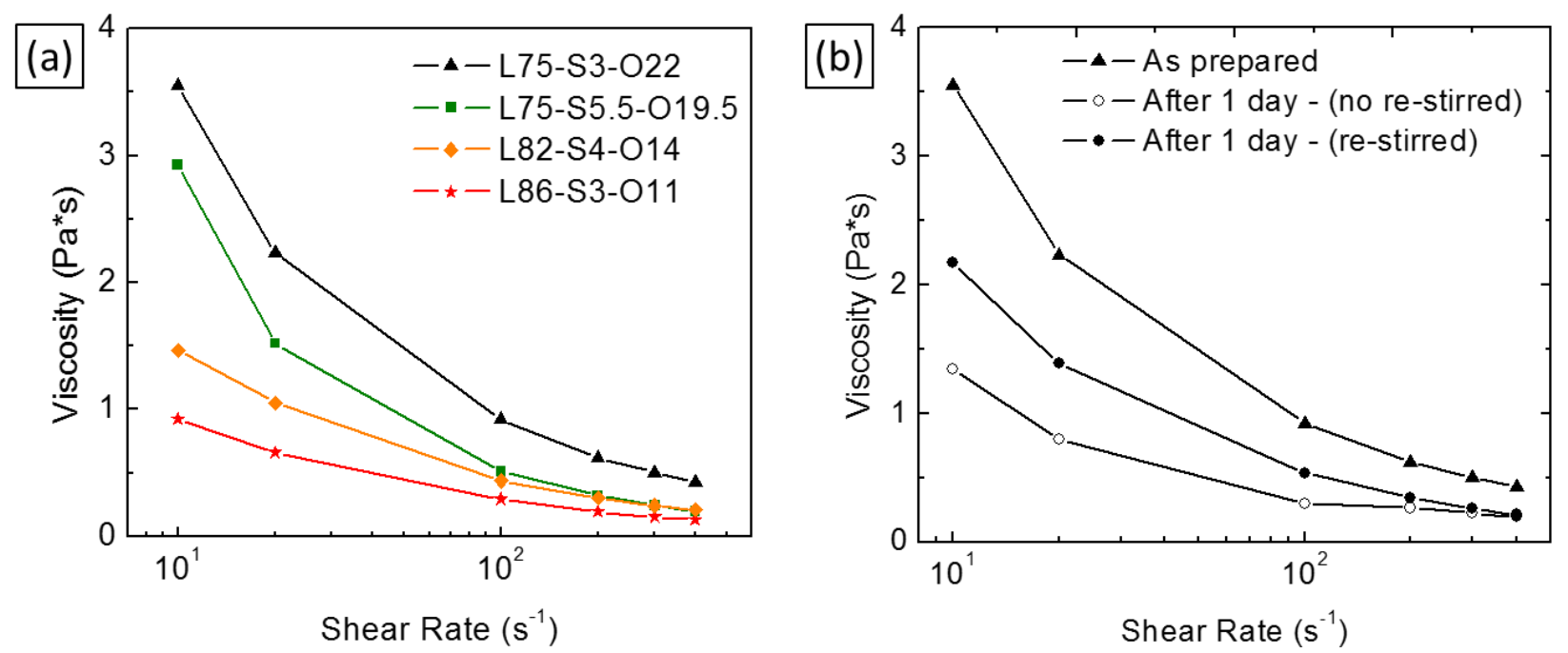

Figure 48. Viscosity of the 1:12 TALH:TiO 2 MEA foams (a) with varying liquid-solid-oil L-S-O amounts in vol\%; and (b) L75-S3-O22 foam as prepared and after 1 day of preparation with and without re-frothing. Frothing time of $4 \mathrm{~min}$.

The frothing time is also an important parameter to consider when targeting for specific viscosities; these are observed to increase by one order of magnitude when the frothing time is increased from 4 to $8 \mathrm{~min}$, (see Figure 48 and Figure 49). This frothing time range was determined empirically as it led to foams with appropriate yield-stress characteristics, in preliminary experiments of the foam preparation route. Similarly, the use of different surfactants (MEA vs. TEA), and TALH:TiO 2 ratio is also observed to modify the viscous properties of the foams, as shown in Figure 49.
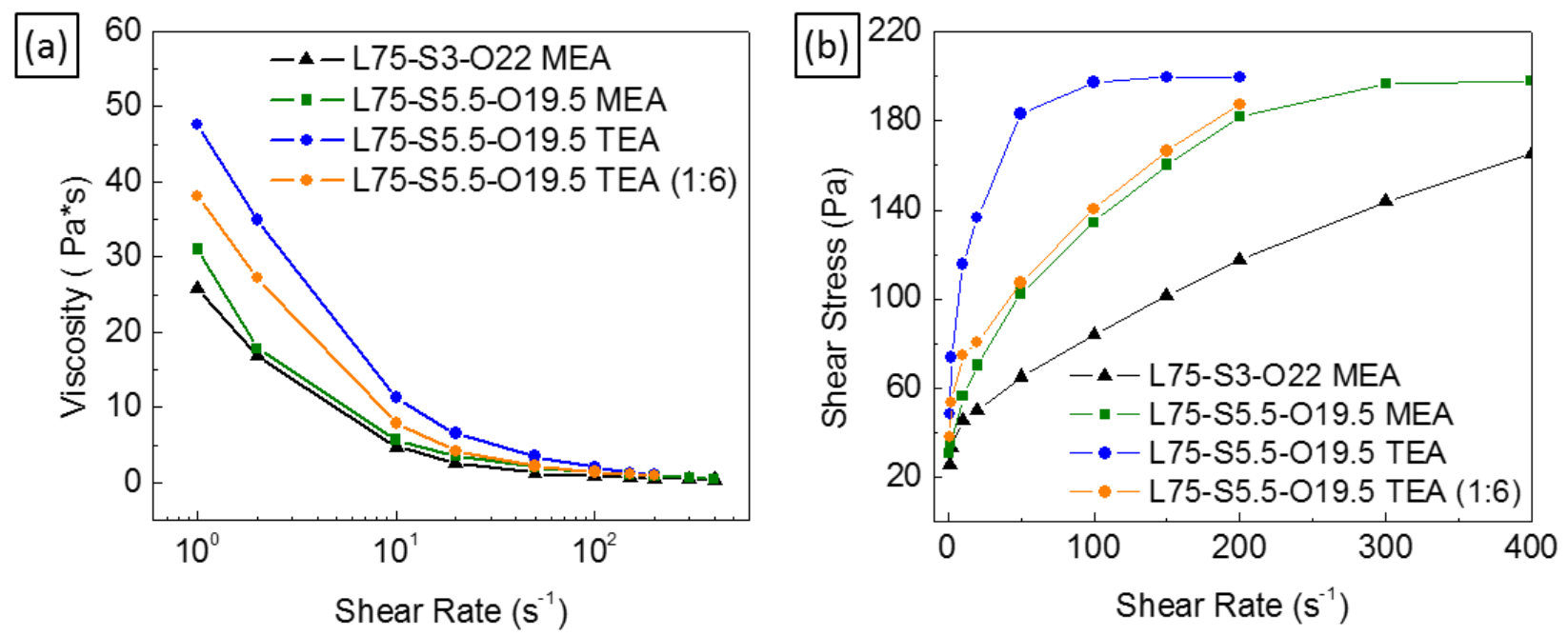

Figure 49. (a) Viscosity and (b) shear-stress as a function of shear-rate for the TALH:TiO $1: 12$ foams after 8 min frothing time from different liquid-solid-oil content (vol\%) formulations. 
Analysis of the shear stress - shear rate relationships of the foams (Figure 49), using the Herschel-Bulkley model (see eq. 1), gives $k$ and $n$ coefficients as indicated in Table 16Table 16, and are indicative of shearthinning behavior, since the flow index $\mathrm{n}$ takes values between 0 and 1 .

Table 16. Herschel-Bulkley coefficients for the 8 min frothed foams.

\begin{tabular}{lccc}
\hline Foam Ink* & $\boldsymbol{\tau}_{\mathrm{y}}$ & $\mathbf{k}$ & $\mathbf{n}$ \\
\hline L75-S3-O22 MEA & 41.5050 & 0.5528 & 0.9241 \\
L75-S5.5-O19.5 MEA & 62.9610 & 0.6741 & 0.9503 \\
L75-S5.5-O19.5 TEA & 100.9900 & 9.5214 & 0.6232 \\
L75-S5.5-O19.5 TEA (1:6) & 59.5970 & 2.5206 & 0.7454 \\
\hline
\end{tabular}

*Amount of liquid-solid-oil (L-S-O) in vol\%; (1:6) refers to TALH:TiO ${ }_{2}$ mole ratio.

In the investigated foams systems, lower shear-stress values $\left(\tau_{y}\right)$ are observed for the foams with lower $\mathrm{TiO}_{2}$ primary particles content. Foam flow exhibiting both solid-like and liquid-like behavior, relates the yield stress with the foam's morphology, especially for dry foams; and to typically decrease as the liquid volume fraction is increased[237]. Additionally, it is observed that the type of surfactant used (i.e., MEA or TEA) also affects the foams' rheology, leading to higher yield stress values. Other aspects of solid-like behavior are a finite shear modulus and slip[238] at solid surfaces. Liquid-like aspects are a shear-thinning viscosity and time-dependent properties. Unless stabilized, the bubbles can collapse[239], reducing the volume of the initially frothed system as time elapses[237]. The compressibility of the foams is also an important factor that influences its rheology and is mostly responsible for the foam's elastic properties upon compression stress application - such as that exerted during CDW (while in the cartridge and while being extruded trough the nozzle). Theories of foam flow predict that, in shear flow, the foam viscosity $\eta$ is generally given by an elastic and a plastic component. The latter arises due to dissipation in the liquid film, and its value is proportional to the viscosity of the liquid phase. This term is significant only for wet foams or those formulated with extremely viscous liquids. The elastic component typically increases as the gas volume fraction is increased [237].

\subsection{Foam Printing}

Among the possible foam compositions, CDW of those with lower water:oil ratios (i.e. 3.42 and 3.86) were investigated and are summarized in Figure 50 (a). The fabrication of planar, 3D free-standing and spanning structures is demonstrated, see Figure 50 (b). Spanning structures for a distance up to $5 \mathrm{~mm}$ are observed to retain their shape, while slightly adhering to the hydrophilic glass edges. Printing of spanning structures on hydrophilic and pre-treated substrates with hydrophobic coatings show a difference on their substratewetting, being greater for hydrophilic substrates. This difference is expected because of the large amount of aqueous phase in the foam formulation, and proves useful for controlling the ink/substrate interactions. Despite of the surface treatment, adhesion of the foams to the substrates was favorable, and is attributed to the amphiphilic nature of the foam. 

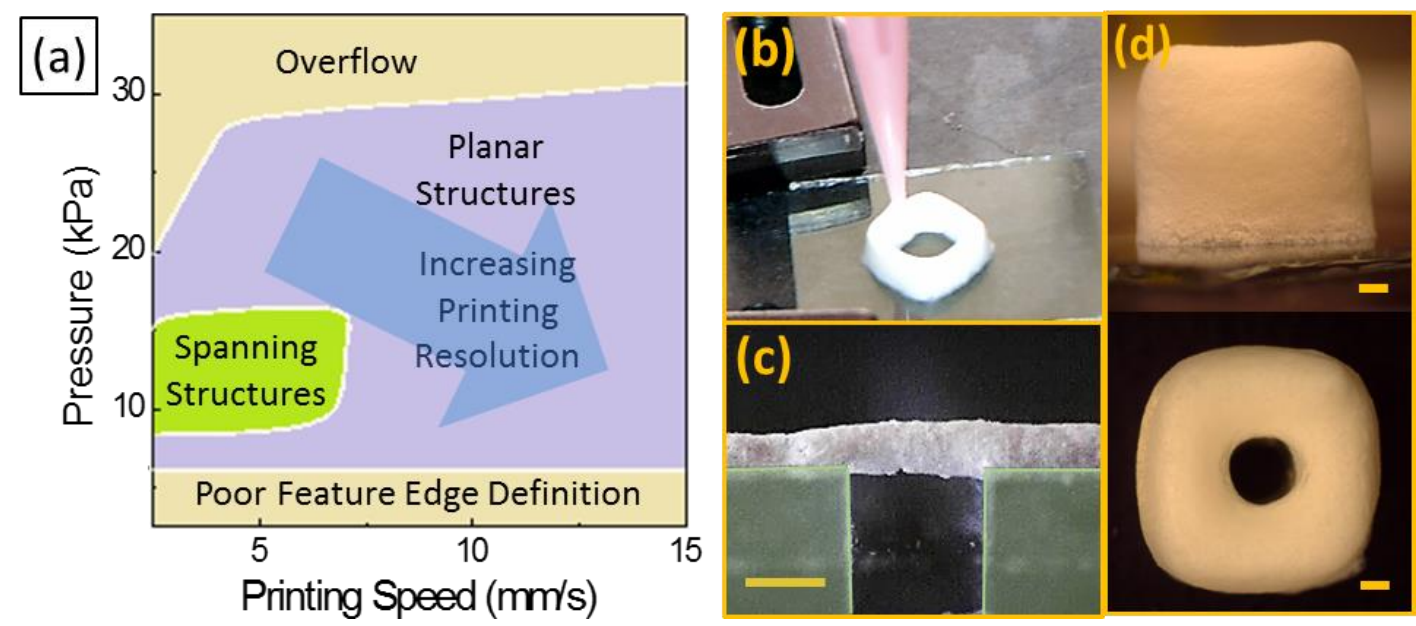

Figure 50. (a) Printing space for the investigated $\mathrm{TiO}_{2}$ foam systems using $580 \mu \mathrm{m}$ nozzles inner diameter at 500 um dispensing height. Printing 3-dimensional layered hollow columns (b) and (d), and spanning foam structures (c), at $9.6 \mathrm{kPa}$ and $5 \mathrm{~mm} / \mathrm{s}$ on hydrophobically treated glass. Scale bars are $1 \mathrm{~mm}$ long.

Generally, for CDW, the ink formulations should display shear-thinning yield-stress behavior which allows for the structures to be formed/extruded, and retain their shape once printed. The yield stress values for the investigated foam systems range from $\sim 40$ to $100 \mathrm{~Pa}$ (see Table 16), allowing their extrusion and shaping at relatively low pressures. These values are comparable to those obtained for ink systems used to fabricate mullite spanning structures[22], and lay around the lower limit of the values range reported as necessary for inks to withstand their own weight across a spanning distance without collapsing, typically ranging from 1 to $1000 \mathrm{~Pa}[22,91,133,134,145]$. The differences in the value ranges reported in literature and the studied foams are viewed with respect to the differences between the foam system and those reported for gel systems [134]. Furthermore, fundamental differences exist between the porous structure of the foam ink and those reported for degassed slurries.

The printing fidelity is mainly affected by the speed and pressure (for a given dispensing nozzle size and dispensing height). From the printing ranges studied for the investigated foams systems, three main regions are obtained: (1) overflow, at relatively high dispensing pressures and lower speeds, due to the high volumetric flow rate of the foams; (2) the printable region, confined between $\sim 6 \mathrm{kPa}$ and $\sim 20$ to 28 $\mathrm{kPa}$ of dispensing pressure; and (3) the poor-edge definition region, at pressures below $\sim 6 \mathrm{kPa}$, where the fidelity of printing is compromised yielding wavy-shape features. The latter are attributed to inconsistent flow and dimensional filament changes as the printing progresses. Spanning structures can be fabricated at the lower speed range of the printable region (i.e. $\sim 1-7 \mathrm{~mm} / \mathrm{s}$ ), while maintaining intermediate levels of dispensing pressure. The spanning structures' integrity at such ranges, may be attributed to the extrudate being structurally coherent and tensile stressed to a relatively small extent. When printing at higher speeds, the extrudate is elongated enough for the gas and oil phases to significantly deform and eventually collapse. Nevertheless, printing of planar foam structures can be realized for a significantly wider range of printing conditions. Accordingly, the printing resolution (i.e. line width) generally increases as speed is increasing and pressure is decreasing within the planar printing space. The resolution of the printed features is typically improved up to a nozzle diameter in width. 


\subsection{Composition, Microstructure and Curing Treatments}

Different means of control of the foams' pore size, configuration (as open- or closed-cell), and surface area properties have been identified via the emulsions' composition. The viscosity and therefore, the mobility of the emulsion's liquid phase as the frothing progresses, is affected by the amounts of solids ( $\mathrm{TiO}_{2}$ primary particles) and solvent present in the foams. This is yielding open-cell foam structures as the solvent amount is increased with respect to the amount of $\mathrm{TiO}_{2}$ primary particles, i.e. larger $\mathrm{L}: \mathrm{S}$ ratio; and conversely closed-cell foams as this ratio is decreased, see Figure 51 (a-c) open-cell vs. (d-i) closed-cell, respectively. Similarly, the choice of surfactant (i.e. MEA or TEA), exhibiting different viscosities and $\mathrm{pK}_{\alpha}$ properties, affects the foam's viscosity and mobility, resulting in slightly different foam microstructures (Figure 51, d-i). For instance, while keeping the L:S ratio constant slightly larger macropores can be distinguished when using MEA, compared to TEA. The latter in particular, aids towards higher ink viscosities (see Figure 49), highly desirable for 3D printing. Additionally, the larger macro-pores of the foams - corresponding to trapped air bubbles - are observed to be dependent on the amount of $\mathrm{TiO}_{2}$ primary solids, see Figure 47 and Figure 51, where the macropore sizes are larger for the foams with lower $\mathrm{TiO}_{2}$ primary solids. The later result, suggests that such formation is strongly influenced by the particle aggregation behavior, observed in previous studies of the $\mathrm{TiO}_{2}: \mathrm{TALH}$ system [128]. Specifically, it may be the case that the $\mathrm{TiO}_{2}$ nanoparticles tend to aggregate at the periphery of the gas bubbles, and when high in concentration, lead to macro-pore size suppression as the solvent is evacuated. Similar relationships between viscosity and foam pore size distribution have been reported for $\mathrm{pH}$-particle stabilized $\mathrm{Al}_{2} \mathrm{O}_{3}$ foam systems $[168,240,241]$ with the $\mathrm{pH}$ values up to $5.5[10,154]$; our foams on the other hand exhibit $\mathrm{pH}$ values ranging from 6 to 7 . The foam prepared from the (1:6) TALH:TiO 2 ratio aqueous suspension, is also leading to rather open-cell configurations, strengthening the latter observation since there is more Ti-organic with respect to $\mathrm{TiO}_{2}$ primary particles, and therefore the inter-particle interactions are affected. 


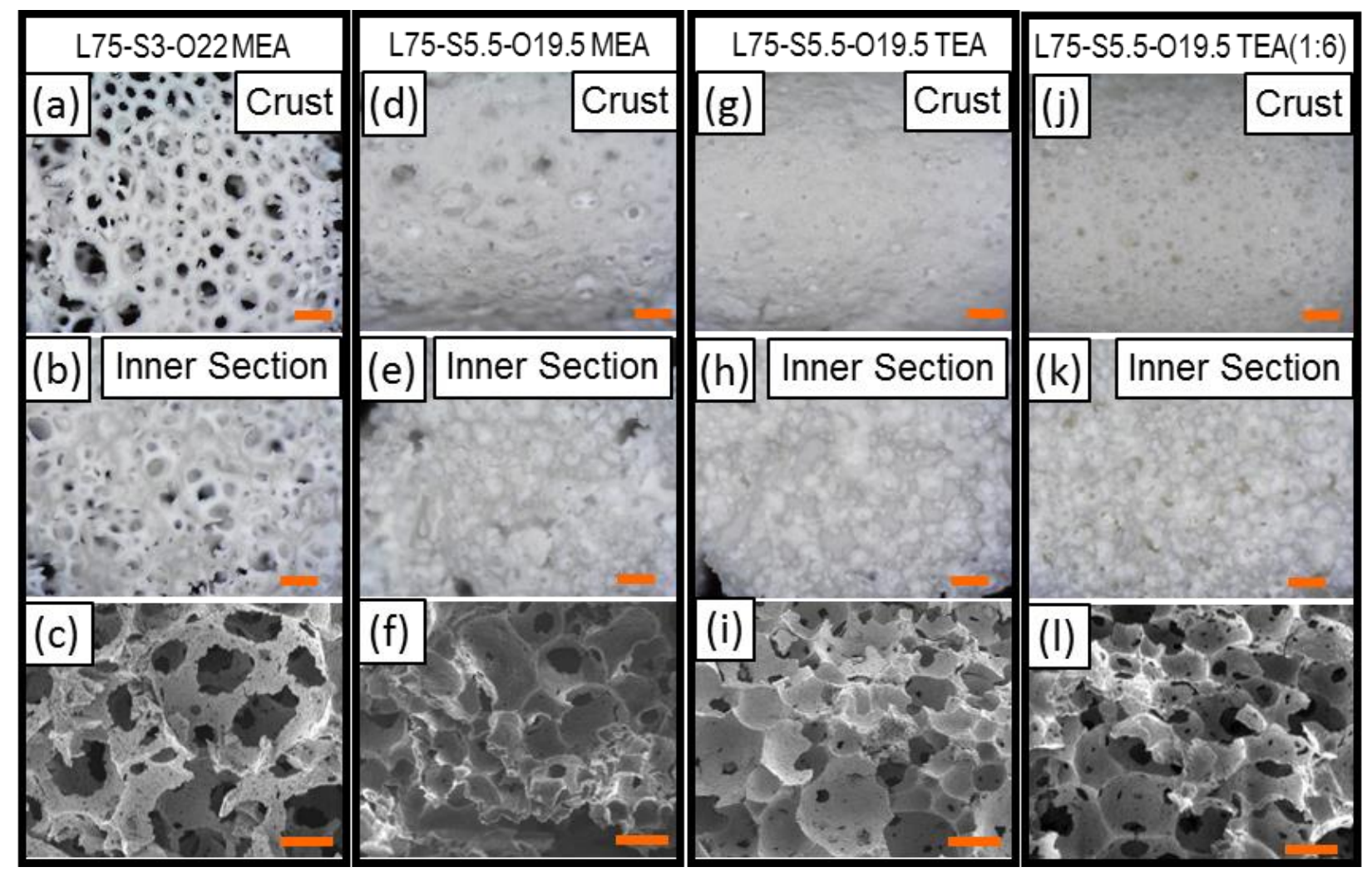

Figure 51. Low-magnification optical microscope and SEM images of the open-cell (a-c) and (j-l); and closed-cell foams (d-i). Printing direction is from right to left. Scale bars correspond to $200 \mu \mathrm{m}$ for optical microscope images, and to $100 \mu \mathrm{m}$ for SEM images (c, $f, i$, and $I)$.

The formation of a crust in the printed foams, common to all foams prepared, exhibits smaller pore sizes when compared to the inner regions of the foams. Such crust is characteristic of solvent drainage within the foam[236], coupled with rapid solvent evaporation at the printed structures' outer surface. A slight elongation of the pores along the printing direction can be distinguished at the crust surface for the foams with higher $\mathrm{TiO}_{2}$ content (Figure 51. d, g, and j), which is attributed to the effects of the shear stress exerted on the foams while printing. This is ultimately providing further means to induce some degree of ordering in these mesoporous structures, as it is known that paste-like systems exhibit plastic memory when subjected to externally applied stresses[242]. In particular, alignment of dispersed nanomaterials using CDW has been reported as to occur when their characteristic size is comparable to the dispensing nozzle diameter [243]. It is then proposed, that the investigated foams being colloidal suspensions, can be considered as similar systems, where the "nanomaterials" are replaced by gas bubbles or micellar formations of the oil-aqueous $/ \mathrm{TiO}_{2}$ mixture. Nevertheless, at the inner regions of the foams, the drained solvent contributes to the relaxation and coalescence of inner gas bubbles, after the shear stress is applied during printing. SEM observation of the inner surface of the foams' pores show similar $\mathrm{TiO}_{2}$ particle assembly characteristics irrespectively of the foam morphology, i.e. open- or closed-cell. However, slightly rougher inner macro-pore surfaces are observed for the L75-S5.5-019.5 MEA foam (see Figure 52), which correlates with the difference in the measured BET surface area (Table 18). This can be used to further control the microstructure of these foams systems based on choice of emulsifiers among others. 

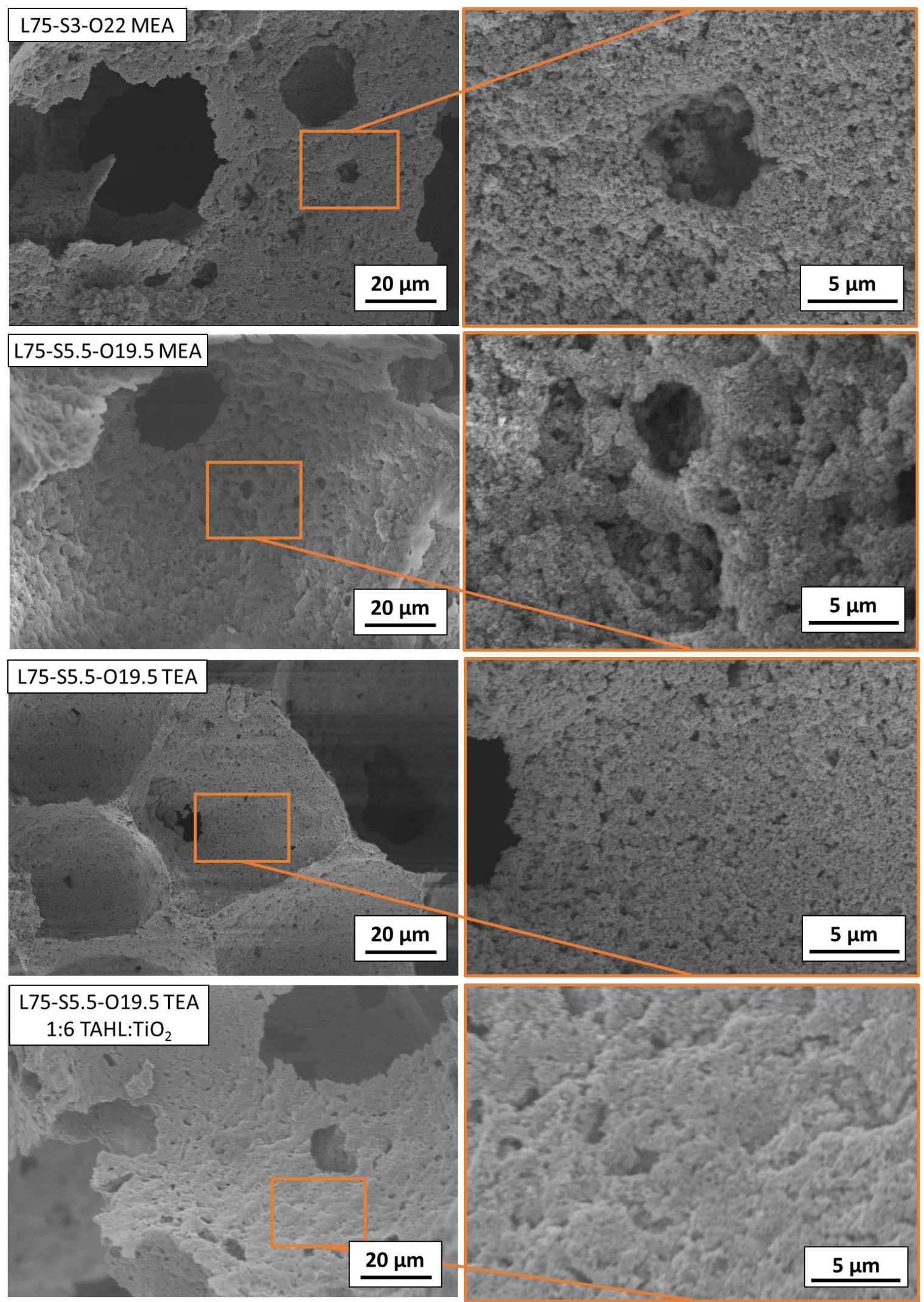

Figure 52. Scanning electron microscope images of the macro-pores inner surfaces for the different foams systems studied and sintered at $500^{\circ} \mathrm{C}$. 
The effects of tuning the microstructure and morphology of the foams can also be observed in their postprocessing (i.e. sintering), surface properties and functional performance (e.g. heterogeneous photocatalysis, see Figure 55). TGA curves of the different foams (Figure 53.) exhibit inflection points at $\sim 150^{\circ} \mathrm{C}, 350^{\circ} \mathrm{C}$ and $450^{\circ} \mathrm{C}$. These correspond in order to: end of solvent evaporation, organics decomposition (i.e. $\mathrm{TALH}$ to $\mathrm{TiO}_{2}$ transformation and oil phase decomposition), and amorphous $\mathrm{TiO}_{2}$ to anatase phase transformation, in agreement with previous observations for similar $\mathrm{TiO}_{2}-\mathrm{TALH}$ systems.[128] The studied formulations yield different amounts of $\mathrm{TiO}_{2}$ depending on their constituents.

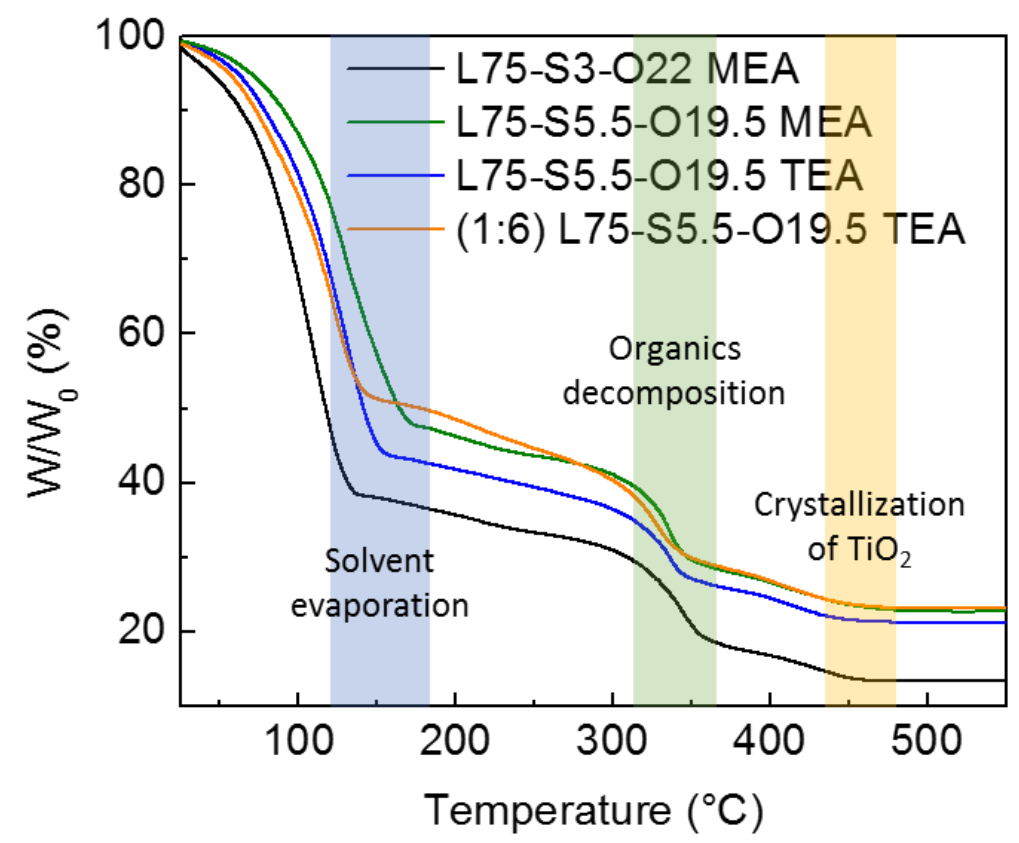

Figure 53. Thermo-gravimetric analysis profiles for the different foam formulations.

The atom economy ( $\mathrm{AE}$ ), defined as the \% mass ratio between the target compound (material) and its precursors [244,245], can be estimated from the materials design and the TGA results, see Table 17. Thus, the foams formulated with higher amounts of primary $\mathrm{TiO}_{2}$ particles $(5.5$ vol\%) - corresponding to $18.17 \%$ of the total weight of the initial wet-foams, yield $\sim 21.95 \%$ of $\mathrm{TiO}_{2}$ after sintering as measured using TGA, (19.68 wt\% theoretical, also AE). Similarly, the foams with smaller amounts of primary $\mathrm{TiO}_{2}$ particles ( 3 vol\%), corresponding to $9.91 \mathrm{wt} \%$ of the initial wet-foams, yield $~ 13.31 \mathrm{wt} \%$ of $\mathrm{TiO}_{2}$ solids after sintering (TGA measured); with a theoretical AE of 10.74 wt\%. The increase in $\mathrm{TiO}_{2}$ after sintering is expected because the TALH (Ti-organic complex), is also transformed into $\mathrm{TiO}_{2}$. Here, the choice of $\mathrm{TAHL}_{\mathrm{TiO}}$ ratio significantly affects the yield of $\mathrm{TiO}_{2}$ solids, increasing to $23.13 \%$ (TGA measured) when using 1:6 $\mathrm{TALH}: \mathrm{TiO}_{2}$ mol ratio (theoretical AE of $24.23 \%$ ). The sintered foams correspond to $92.3 \mathrm{wt} \% \mathrm{TiO}_{2}$ primary particles and $7.7 \mathrm{wt} \% \mathrm{TiO}_{2}$ from TALH for the 1:12 $\mathrm{TAHL}_{\mathrm{TiO}} \mathrm{TiO}_{2}$ mol ratio formulations; and to $75 \mathrm{wt} \% \mathrm{TiO}_{2}$ primary particles and $25 \mathrm{wt} \% \mathrm{TiO}_{2}$ from $\mathrm{TALH}$, for the $1: 6 \mathrm{TALH}: \mathrm{TiO}_{2}$ mol ratio formulations, respectively. Therefore, it is found that the $\mathrm{TALH}: \mathrm{TiO}_{2}$ ratio has a great effect in changing the foams' surface properties as observed from the BET measurements (see Table 18). Additional sources of variability between the expected $\mathrm{TiO}_{2}$ solids yield and the experimentally obtained, may be attributed to the loss of weight due to solvent volatilization during frothing. 
Table 17. $\mathrm{TiO}_{2}$ sources in wt\% present in the wet foams, the targeted sintered structures (theoretical), and the experimental yield as calculated from TGA.

\begin{tabular}{|c|c|c|c|c|c|c|c|c|}
\hline \multirow[b]{2}{*}{ Foam } & \multicolumn{2}{|c|}{ Wet Foam } & \multicolumn{5}{|c|}{ Sintered Foam (theoretical) } & \multirow{2}{*}{$\begin{array}{l}\text { Sintered Foam } \\
\text { TGA yield } \\
\text { (wt\%) }\end{array}$} \\
\hline & $\begin{array}{c}\text { Primary } \\
\mathrm{TiO}_{2} \\
(\mathrm{wt} \%)\end{array}$ & $\begin{array}{l}\text { TALH } \\
\text { (wt\%) }\end{array}$ & $\mathrm{TiO}_{2}$ & $\begin{array}{c}\% \text { of } \\
\text { composite } \\
\text { (Primary) }\end{array}$ & $\begin{array}{c}\mathrm{TiO}_{2} \\
\text { From TALH }\end{array}$ & $\begin{array}{c}\% \text { of } \\
\text { composite } \\
\text { (TALH) }\end{array}$ & $\begin{array}{c}\mathrm{TiO}_{2} \text { from } \\
\text { combined } \\
\text { sources }\end{array}$ & \\
\hline L75-S3-022 MEA & 9.91 & 3.04 & 9.91 & 92.3 & 0.83 & 7.7 & 10.74 & 13.32 \\
\hline L75-S5.5-019.5 MEA & 18.17 & 5.58 & 18.17 & 92.3 & 1.51 & 7.7 & 19.68 & 22.66 \\
\hline L75-S5.5-019.5 TEA & 18.17 & 5.58 & 18.17 & 92.3 & 1.51 & 7.7 & 19.68 & 21.24 \\
\hline (1:6) L75-S5.5-019.5 TEA & 18.17 & 11.15 & 18.17 & 75.0 & 6.06 & 25.0 & 24.23 & 23.13 \\
\hline
\end{tabular}

Dimensional changes associated with sintering of ceramics are usually expected to occur and still need further understanding on a fundamental level[145]. Here it is believed, that by dispersing the oil phase to an already continuous aqueous phase - containing the $\mathrm{TiO}_{2}$ primary particles and $\mathrm{TALH}$ - the aqueous network remains coherent. During frothing, the oil droplets become smaller and aid in the stabilization of the gas bubbles; which in turn may serve as pressure reservoirs influencing the volatilization of the solvent in the foam cell walls. Then, as the solvent is evacuated, the oil-stabilized primary particles tend to agglomerate and remain suspended in the predominantly oil scaffold. The melting temperature of the oil phase may play an additional role, the higher the melting temperature of the oil phase, the stronger the oily scaffold it forms as it becomes solid at room temperature. Here the cells - when open - serve as solvent evacuation pathways, which can diffuse towards the outer surface of the printed object (crust), or towards the inner cell cavities. The extra surface for solvent evaporation, provided by the open-cell microstructures, helps to equilibrate rates of solvent diffusion (within the colloidal suspension towards the evaporation front), and the evaporation rate at the liquid-gas interface, which has been reported as a key mechanism to prevent cracking of ceramic colloidal films.[246] Next, upon sintering, further shrinkage occurs when the oil phase is eliminated. However, the aggregation forces between the particles prevent the collapse of the foam structure by self-locking the primary particles thus limiting their relative displacement as sintering progresses. Printed hollow open-cell column shrinkage is found to lay around $16 \%$ in all directions $(x-y-z)$; whereas closed-cell foam configurations exhibited a greater shrinkage in the $z$-direction of $\sim 25 \%$. In the case of planar structures (printed films of $2 \mathrm{~cm} \times 1 \mathrm{~cm}$ ), anisotropic shrinkage is observed due to the constrained $x$ and $y$ bonding of the printed layer to the substrate, this change is approximately $25 \%$ in the $z$-direction for open-cell structures and $37 \%$ for closed-cell structures, respectively. The greater dimensional change experienced by the foams with closed-cell structures is attributed to the effects of the inter-particle forces, being larger for higher particle concentration formulations. These are weaker for open-cell architectures, as opposed to closed-cell foams, where the $\mathrm{TiO}_{2}$ primary content is higher, since the compressive yield-stress associated to volumetric changes is known to be strongly dependent on the particle-particle interactions.[246] Additionally, it is postulated that the inner surfaces of the open-cell foams, being larger in relation to the $\mathrm{TiO}_{2}$ wall thickness, as observed using SEM, provide larger stress-release surfaces. Finally, the correlation of the volumetric contraction and the weight decrease after sintering is also indicative of the strong particle-particle interactions influence in the microstructural evolution of these foams. Since it is observed that such 
contraction is minimum for those foams experiencing larger weight decrease with sintering (i.e. L75-S3O22 foams), as observed from TGA.

To explore the microstructural evolution of the printed foams under mild temperature and UV conditions, SEM images of the L86-S3-O11 MEA foam (the one with the least amount of oil phase), after thermal and UV exposure treatments were taken - see Figure 54. The SEM images show the typical assembling morphologies of the $\mathrm{TiO}_{2}$ foams, and the combination of the remaining organic materials with the primary $\mathrm{TiO}_{2}$ particles. It is observed that the transformation of the foams upon different energy sources, results in significant differences in the materials microstructure. Organic-like platelets are observed at the periphery of the macro-pores' openings, at the surface of the samples treated at $150^{\circ} \mathrm{C}$ for 30 min Figure 54 (a). These formations were not observed at the surface of the samples treated under UV light, Figure 54 (b). At the inner regions of the foams, these platelet formations are also observed, and again exhibited significant differences depending on the curing treatment. For the $150^{\circ} \mathrm{C}-30 \mathrm{~min}$ treatment, small clusters of platelet formations can be distinguished. However, for the samples treated using UV light, these formations are present along all the inner pore surfaces. This can be expected because of the UV absorption properties of $\mathrm{TiO}_{2}$; at the foams' surface, the UV light is blocked impairing the light penetration to the inner regions of the foam and making it difficult to decompose the organics in such locations.

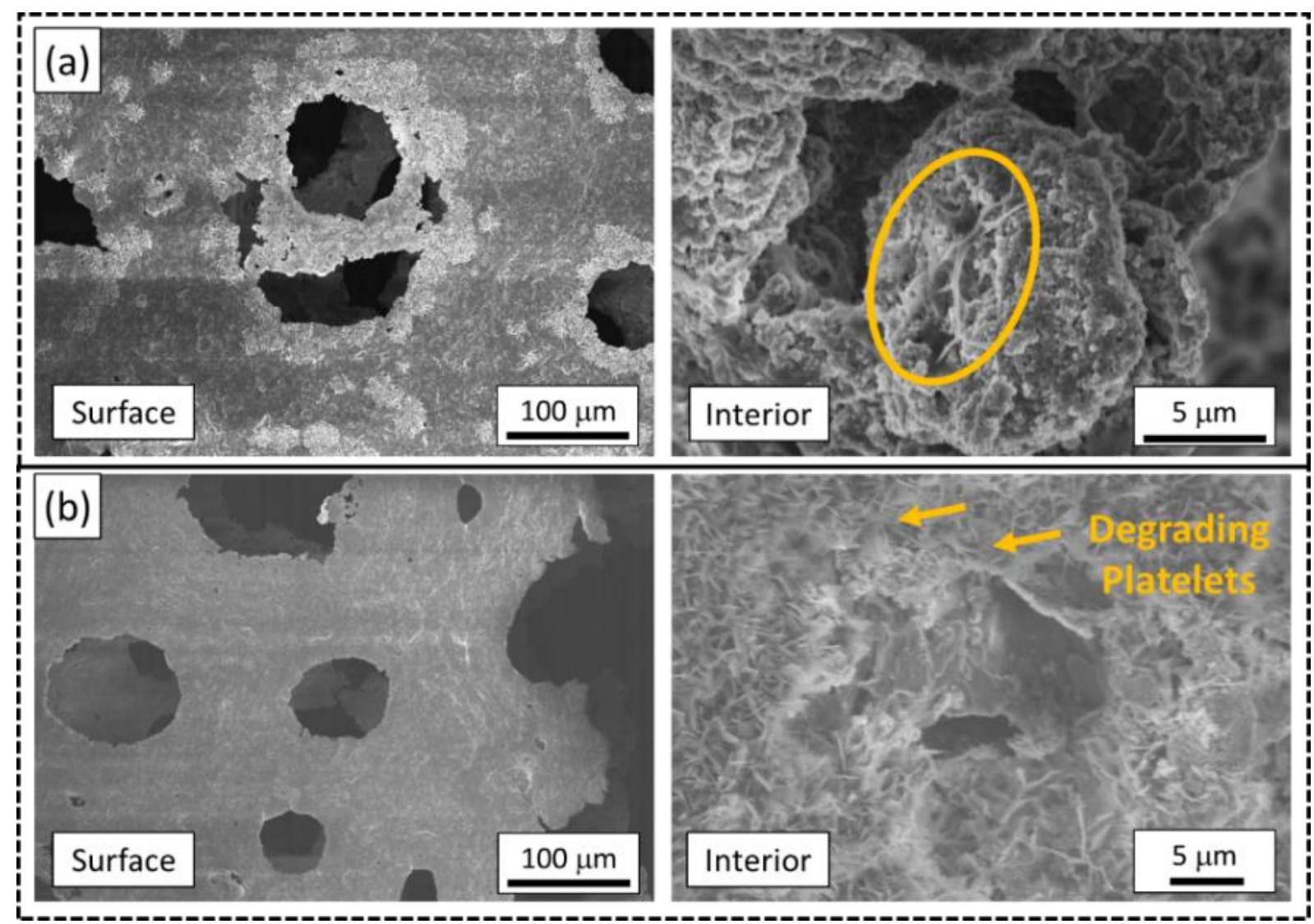

Figure 54. Microstructure of the surface and inner regions of the L86-S3-O11 MEA foam, treated at (a) $150^{\circ} \mathrm{C}-30 \mathrm{~min}$ and (b) $6 h$ UV $(\lambda=254 \mathrm{~nm})$.

As will be latter discussed in this chapter, the differences in the foam cell morphologies greatly affect the photocatalytic behavior of the foams which, when using UV light curing treatments may have significantly 
different results depending on the foam morphology - light diffusion interactions. Therefore, the studied $\mathrm{TiO}_{2}$ foams are also susceptible of use as UV-light protective coatings, which are expected to become stronger upon further UV light exposure, due to the transformation of TALH into $\mathrm{TiO}_{2}$.

\subsection{Microstructural, Compositional and Photocatalytic Relationships}

Photocatalysis results indicate that the foam films with open-cell structures, perform better than their closed-cell counterparts in degrading MB, see Table 18; where the apparent first-order degradation rate constant $k_{\text {app }}$ values are greater (with respect to the respective solids content and surfactant type). This result can be attributed to the inherent ability of open-cell structures to facilitate better circulation of the $\mathrm{MB}$ solution, and their availability for decomposition at $\mathrm{TiO}_{2}$ photoactive surfaces.

Table 18. Apparent first-order degradation rate constant $k_{a p p}$ and Brunauer-Emmett-Teller (BET) surface area. *Amount of liquid-solid-oil (L-S-O) in vol\%.

\begin{tabular}{lcc}
\hline \multicolumn{1}{c}{ Foam* } & $\begin{array}{c}\boldsymbol{k}_{\text {app }} \\
\times 1 \mathbf{0}^{-2} \mathbf{~ m i n}^{-1}\end{array}$ & $\begin{array}{c}\text { BET Surface Area } \\
\mathbf{m}^{2} / \mathbf{g}\end{array}$ \\
\hline Blank & 0.103 & --- \\
L75-S3-O22 MEA & 0.418 & 46.094 \\
L75-S5.5-O19.5 MEA & 0.262 & 49.556 \\
L75-S5.5-O19.5 TEA & 0.205 & 46.766 \\
L75-S5.5-O19.5 TEA (1:6) & 0.269 & 37.218 \\
Primary TiO $_{2}$ Particles & --- & 46.171 \\
\hline
\end{tabular}

The heterogeneous photocatalytic degradation of $\mathrm{MB}$ over time, is included in Figure 55 . In the case of closed-cell structures, the $\mathrm{TiO}_{2}$ network is more compact, thus inhibiting the diffusion of UV light through inner regions of the film. On the other hand, the open-cell structures allow a greater depth of UV light diffusion. In addition, it is observed that the films with closed-cell structures (i.e. L75-S5.5-019.5 MEA and TEA), exhibit similar performance, suggesting no significant effects from the use of MEA or TEA as surfactants, but rather stronger dependence on the macropore size - light interactions.

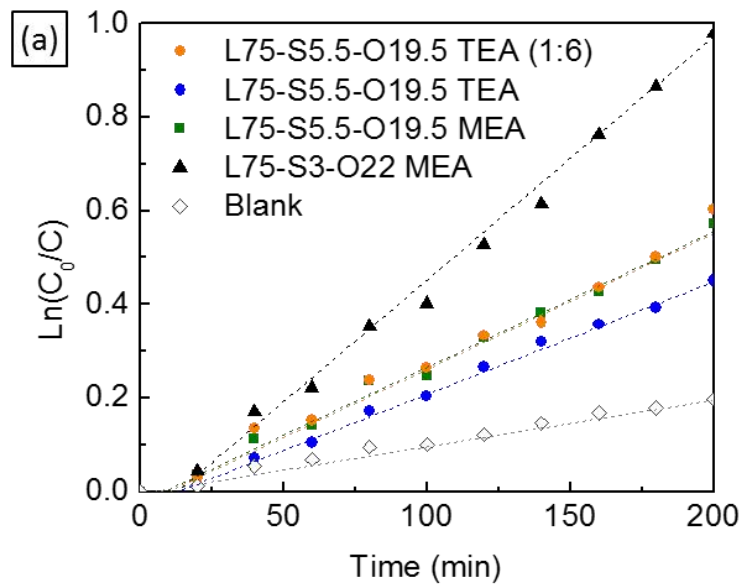

(b)

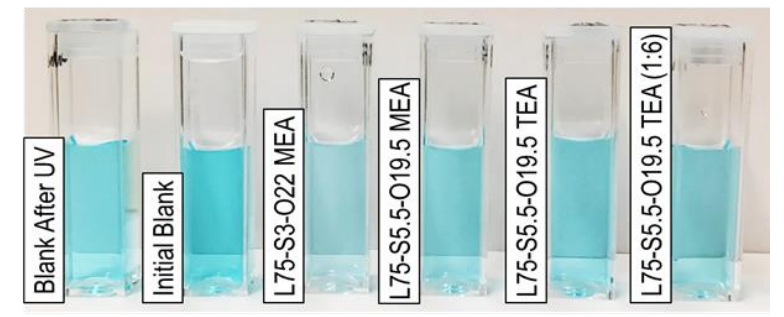

Figure 55. (a) Linearized methylene blue concentration change in time, undergoing heterogeneous photocatalytic degradation in the presence of the different $\mathrm{TiO}_{2}$ foams under UV light exposure at $\lambda=254 \mathrm{~nm}$. (b) Photograph of cuvettes with degraded methylene blue solutions after 200 min of UV exposure. 
A slight improvement is noticed for the 1:6 TALH:TiO 2 TEA formulation with respect to its 1:12 counterpart; again suggesting a stronger photocatalytic activity dependence on the circulation properties than on the amount of measured Brunauer-Emmet-Teller (BET) surface area. Specifically, a correlation between the photocatalytic performance and cell configuration can be established, since such performance is maximized for open-cell architectures, despite exhibiting generally lower surface area. Figure 56 (a), includes the Barrett-Joyner-Halenda (BJH) cumulative surface area and pore area distributions, which support this observation. The photocatalytic activity of the foams is comparable to similar mesoporous $\mathrm{TiO}_{2}$ films[38] and $\mathrm{TiO}_{2}: \mathrm{TALH}$ systems [128]. The latter exhibiting remarkably good performance due to the low specific energy treatments, which prevent their surface coarsening.
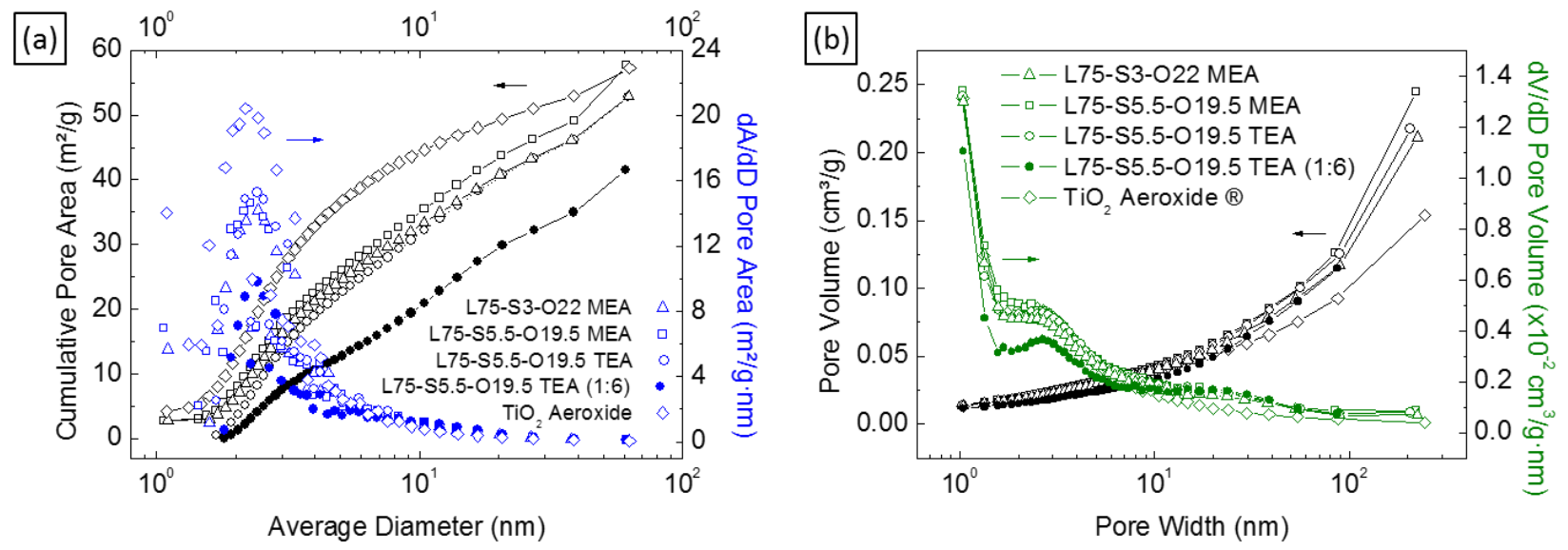

Figure 56. (a) Barrett-Joyner-Halenda (BJH) cumulative micro-pore area (black) and micro-pore area distribution (blue); and (b) Horvath-Kawazoe micro-pore volume (black) and micro-pore volume distribution (green), for the primary TiO2 nanoparticles and studied foam systems. The amount of liquid-solid-oil (L-S-O) is in vol\%.

Additionally, roughening or smoothening of the surface of the $\mathrm{TiO}_{2}$ primary particles (and consequently foam walls), can be induced based on the nucleation and crystallization of secondary $\mathrm{TiO}_{2}$ from TALH[128]. Porosimetry measurements (see Figure 56(b)), show no significant difference in the overall pore size distribution for all the foams studied (when keeping the same TALH:TiO 2 ratio) and the primary $\mathrm{TiO}_{2}$ nanoparticles. The rather invariable character of these distributions, suggest that the microporosity of the foams is driven by the primary nanoparticles' concentration, size and surface properties. The pores' diameters span from $\sim 1$ to $250 \mathrm{~nm}$, comprising micro- meso- and macro-porosity regimes. Nevertheless, the variation of the TALH:TiO 2 ratio may be used to modify such distributions; for the 1:6 TALH:TiO $\mathrm{L}_{2}$ S5.5-019.5 TEA foam, the surface area decreased with respect to all previous foam systems (with 1:12 $\mathrm{TAHL}: \mathrm{TiO}_{2}$ mole ratio) and to the $\mathrm{TiO}_{2}$ primary particles; while reducing the porosity in the micro- and meso-pore regimes.

XRD studies, confirm the primary particles' properties to drive the microstructural characteristics of the foams, where the $\mathrm{TiO}_{2}$ crystallite size show no significant coarsening even after the $500^{\circ} \mathrm{C}$ sintering process (see Table 19). The broadening of the XRD characteristic peaks with respect to those of the primary $\mathrm{TiO}_{2}$ particles, is attributed to the formation of nanocrystallites from the TALH transformation into $\mathrm{TiO}_{2}$ which lowers their average size, as reported previously[128]. The change in crystallite size for the 1:6 TALH: $\mathrm{TiO}_{2}$ foam formulation, shows an increase of about 11.4 and $3.4 \%$ for the rutile and anatase crystallites, respectively. The latter result, contrasts those for the $\mathrm{TALH}: \mathrm{TiO}_{2} 1: 12$ systems which exhibited 
a general decrease up to $\sim 6.8 \%$, see Table 19. This trend corresponds with the observations from the studies of the aqueous TALH:TiO 2 suspensions (chapter 5 ), where the 1:6 systems favor the growth of crystallites, whereas for all the other studied concentrations the crystallite size exhibited a general decrease.
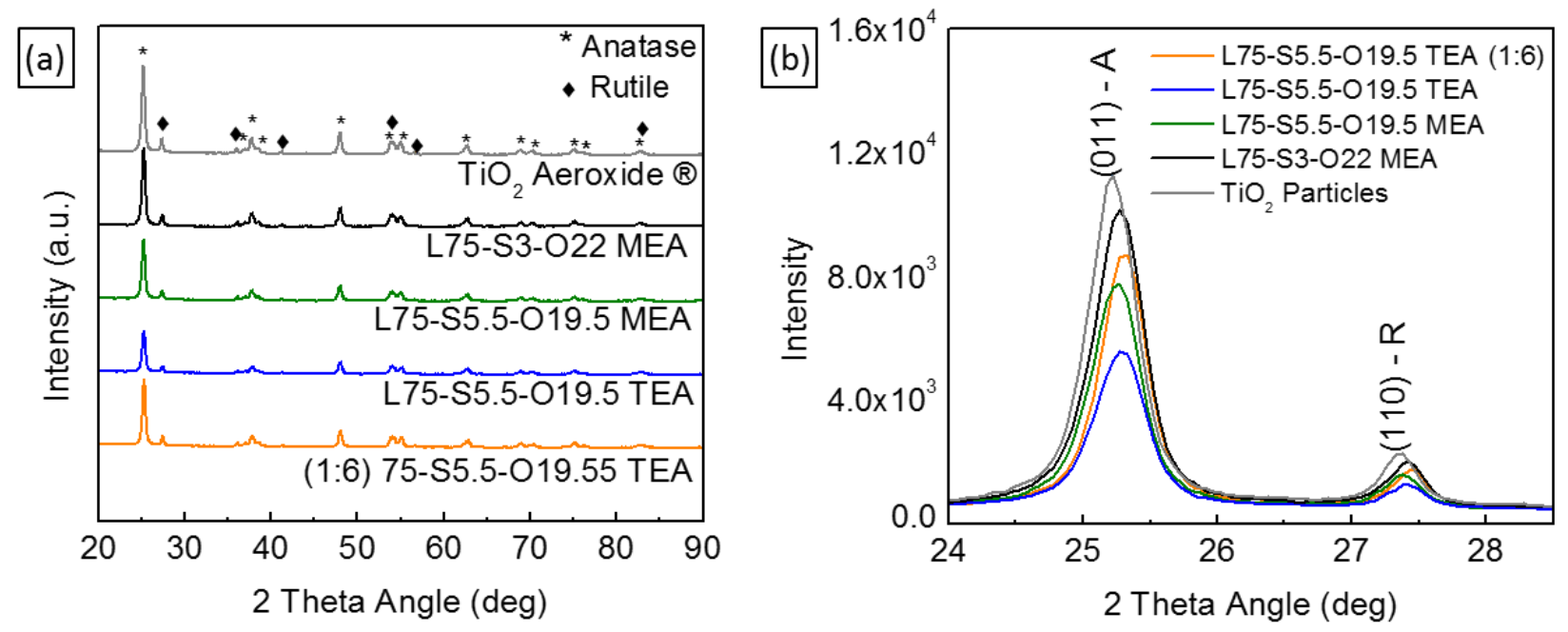

Figure 57. X-ray diffraction patterns of the studied foams and primary $\mathrm{TiO}_{2}$ nanoparticles; (b) main diffraction peaks corresponding to anatase and rutile crystalline structures. The amount of liquid-solid-oil (L-S-O) is in vol\%. (1:6) refers to $\mathrm{TALH}: \mathrm{TiO}_{2}$ mole ratio.

Table 19. Amount of anatase and rutile phases, and crystallite size estimations from XRD data using Gaussian peak fits. The amount of liquid-solid-oil (L-S-O) is in vol\%. (1:6) refers to TALH:TiO 2 mole ratio. ${ }^{*}$ Calculated using the model by Gribb et al[208].

\begin{tabular}{|c|c|c|c|c|c|c|}
\hline \multirow[b]{2}{*}{ Foam } & \multicolumn{2}{|c|}{$\begin{array}{l}\text { Crystalline Phase } \\
\text { Amount* (wt.\%) }\end{array}$} & \multicolumn{2}{|c|}{$\begin{array}{c}\text { Grain Size }(\mathrm{nm}) \\
\text { Scherrer's formula }\end{array}$} & \multicolumn{2}{|c|}{$\begin{array}{c}\Delta \text { Grain Size (\%) } \\
\text { w.r.t. } \mathrm{TiO}_{2} \text { Aeroxide }\end{array}$} \\
\hline & Rutile & Anatase & Rutile & Anatase & Rutile & Anatase \\
\hline L75-S3-022 MEA & 10.999 & 89.001 & 55.727 & 17.863 & -0.498 & -4.840 \\
\hline L75-S5.5-019.5 MEA & 11.303 & 88.697 & 52.220 & 18.077 & -6.759 & -3.698 \\
\hline L75-S5.5-019.5 TEA & 11.089 & 88.911 & 55.714 & 17.596 & -0.520 & -6.262 \\
\hline L75-S5.5-019.5 TEA 1:6 & 11.068 & 88.932 & 62.391 & 19.401 & 11.401 & 3.356 \\
\hline $\mathrm{TiO}_{2}$ Aeroxide ${ }^{\circledR}$ & 11.415 & 88.585 & 56.005 & 18.771 & 0.000 & 0.000 \\
\hline
\end{tabular}

Furthermore, the calculated lattice parameters $a$ and $c$, with a standard deviation below $0.0118 \AA$ with respect to those reported in literature[18], display a general decrease - with respect to that calculated for the primary $\mathrm{TiO}_{2}$ particles - that accentuates more as the TALH amount is increased. Such decrease is maximum for the 1:6 formulation and it is attributed to the larger amount of $\mathrm{N}$ available (from TALH). This excess amount of $\mathrm{N}$ may result in interstitial or substitutional doping [247] of the $\mathrm{TiO}_{2}$ in place of $\mathrm{O}$, due to their similar atomic size and electronegativity. The calculated lattice parameters are summarized in Table 20. 
Table 20. Calculated lattice parameters for the $\mathrm{TiO}_{2}$ primary particles, and the resulting $\mathrm{TiO}_{2}$ in the studied foam systems. The amounts of liquid-solid-oil (L-S-O) are given in vol\%; (1:6) refers to TALH:TiO 2 mole ratio.

\begin{tabular}{|c|c|c|c|}
\hline \multirow[t]{2}{*}{ Foam } & \multirow[t]{2}{*}{ Crystalline Phase } & \multicolumn{2}{|c|}{ Lattice Parameter (̊̊) } \\
\hline & & $a$ & c \\
\hline \multirow[t]{2}{*}{ L75-S3-022 MEA } & Anatase & 3.7936 & 9.5179 \\
\hline & Rutile & 4.6007 & 2.9612 \\
\hline \multirow[t]{2}{*}{ L75-S5.5-019.5 MEA } & Anatase & 3.7970 & 9.5274 \\
\hline & Rutile & 4.6059 & 2.9632 \\
\hline \multirow[t]{2}{*}{ L75-S5.5-019.5 TEA } & Anatase & 3.7921 & 9.5115 \\
\hline & Rutile & 4.6007 & 2.9579 \\
\hline \multirow[t]{2}{*}{ L75-S5.5-019.5 TEA 1:6 } & Anatase & 3.7861 & 9.5140 \\
\hline & Rutile & 4.5960 & 2.9581 \\
\hline \multirow[t]{2}{*}{$\mathrm{TiO}_{2}$ Aeroxide $\AA$} & Anatase & 3.8010 & 9.5380 \\
\hline & Rutile & 4.6106 & 2.9633 \\
\hline
\end{tabular}

\subsection{Concluding Remarks}

As can be observed through the analysis of the proposed foam systems, the different compounds utilized for their synthesis are bio-compatible, non-toxic and water-stable. This signifies that the foams' processing does not require special humidity or vacuum conditions, and that there is ample time for these materials' manipulation. Moreover, because of the excellent bio-compatibility of all the foam precursors (besides $\mathrm{TiO}_{2}$ ), the synthesized cellular structures are natural candidates for their bio-functionalization or implementation as biocomponents. This investigation shows how, the developed ceramic foam synthesis approach offers fine tailoring of the morphology, surface area and pore-size distributions; and mass/light transport interactions (photocatalytic performance) based on the careful selection of the TALH:TiO ${ }_{2}$ ratio, $\mathrm{L}: \mathrm{S}: \mathrm{O}$ ratio, and primary $\mathrm{TiO}_{2}$ particle properties. Moreover, this method enables the 3D printing of these hierarchically ordered mesoporous structures as free-standing and spanning architectures by tuning the rheological properties of the yield-stress fluid formulations.

In need of pioneering approaches for responsibly engineered materials and manufacturing, this work demonstrates a practical and relatively simple route to synthesize $\mathrm{TiO}_{2}$ foams (that could be extended to other ceramic materials systems), using colloidal emulsions consisting of a Ti-organic complex $-\mathrm{TiO}_{2}$ particle's suspension as the aqueous phase, and controlling their morphology as printable open- or closed-cell foam architectures. Investigation of their printing space using continuous-flow direct writing is performed, indicating that spanning structures can be fabricated at relatively low writing speeds and intermediate dispensing pressures due to minimum-to-moderate stretching of the printed foam filament. In addition, a potential mechanism for the dimensional change, as the printed foam-inks are transformed into sintered solids, when using heat treatments up to $500^{\circ} \mathrm{C}$ is proposed. Studies of low thermal-energy curing treatments (mild heat and UV) administered to transform the dried foams into higher-degree crystalline structures, show differences in the foam's microstructure, arising from the inherent nature of such energy treatments and the morphology of the treated materials. The differences in photocatalytic performance between open- and closed-cell printed foams are illustrated, and are believed to arise from the electron-photon interactions and macropore size dependence, i.e., from the more efficient circulation 
of the dye solution to the photocatalytically-active sites of the foam, and the improved diffusion of light within the larger macropore open-cell foam structures. Further work on the characterization of these differences, and light - structures' interaction will provide further fundamental insights. The use of inexpensive, innocuous, and bio-compatible materials implemented in these foams, may hold the key for their implementation and scalable 3D printing, enabling a plethora of advanced technological applications. 
Chapter 7: Multiphase Materials Systems II: Encapsulating Materials and Controlling the Nucleation of $\mathrm{Ag}$ on $\mathrm{TiO}_{2}$ Structures

The investigated Ti-organic/ $\mathrm{TiO}_{2}$ foam emulsion system, can be further used for the synthesis and selective positioning of a secondary phase material on the ceramic scaffold, to realize hierarchical mesoporous cellular heterostructures such as metal-ceramic composites. The concept is to use the oil phase of the oil-in-water emulsion system, to encapsulate the secondary phase material or their precursors, and induce their formation by using an external energy source or by using a chemical catalyst. Alternatively, in order to reduce the thermal budget associated to the composites' transformation from the emulsion state to the final functionalized ceramic, the use of hydrocolloids as foam stabilizers[248,249], and as secondary phase materials dispersion medium is proposed. Food hydrocolloids, being widely studied and abundant rheology control compounds[250,251], highly compatible with living organisms, are great substitutional candidates for the realization of environmentfriendly materials. Remarkably, the stabilization role of chitosan polysaccharide hydrocolloids has been reported previously for the fabrication of cellular Si-based soft materials[155].

In the currently proposed multiphase system, two composite groups are envisioned: metal-oxide/metal and metal-oxide/organic-molecule materials; additionally, different metal-oxide-l/metal-oxide-II are also possible. The former (i.e. metal-oxide/metal composites) are widely used for enhanced light harvesting applications including $\mathrm{H}_{2}$ generation[252-257], small molecule detection[16], catalysis[15,52,213,253,255,258-263] and photovoltaics[55,264-266]; as well as for biomedical and antibacterial coatings [267-269]. Similarly, metal-oxide/organic-molecule composites are commonly used in optoelectronic systems such as dye-sensitized solar cells[270], catalysts[34] and biocompatible systems such as scaffolds for tissue engineering [271].

\subsection{Design, Synthesis and Rheology Observations}

To illustrate the proposed concept, metal-oxide/metal composites (i.e. Ag-decorated $\mathrm{TiO}_{2}$ foams) are synthesized. In doing so, alternative methods to fabricate such composites with multiple applications are realized, based on the fundamental insights gathered from investigating these different material systems processing-properties relationships. This work serves as preliminary study for the realization of the more complex ceramic composites. In the described method, the Ag decorations could be included as prepared particles dispersed within the phase complementary to the titania suspension, or they can be formed within such complementary phase from a solution including a Ag-precursor. The complementary phase (oil-based or hydrocolloid-based) serves as controlling agent of the secondary phase (decorative) material nucleation sites (i.e. the Ag particles will nucleate where the encapsulating phase reaches onto the metaloxide structure), and to control the nucleation and growth rate (depending on the viscosity, $\mathrm{pK}_{\alpha}$ properties, and thermal decomposition of such complementary phase). Nucleation of inorganic nanoparticles in oil-in-water emulsions has been demonstrated previously[14]. However, its simultaneous synthesis, with that of mesostructured metal-oxide cellular architectures is herein proposed for the first time.

For the synthesis of Ag-decorated $\mathrm{TiO}_{2}$ foam, the previously studied metal-organic/metal-oxide ( $\mathrm{TALH}: \mathrm{TiO}_{2}$ ) suspension and a $\mathrm{Ag}$ ion-rich oil phase are emulsified, and frothed to incorporate the gas bubbles that lead to macropore features. The only deviation from the plain TALH:TiO ${ }_{2}$ foams synthesis 
procedure, is the triethanolamine (TEA) addition to the Ag acetate ethanol solution to induce the Agprecursor solvation, instead of at the last mixing step before frothing. Once the Ag precursor is solubilized in ethanol, it is added to the oil phase. The ethanol solution mixes readily with the oil phase constituents at $\sim 70{ }^{\circ} \mathrm{C}$, and the blend remains clear and does not change its color. The Ag oil phase is stirred continuously to evaporate the ethanol, and once no volumetric changes are observed, the $\mathrm{TiO}_{2}$ suspension is added and allowed to homogenize by continuous stirring while closed. Once the oil-phase and the titania suspension are visibly blended, frothing with a wisk-like mechanical mixer is performed. The viscosity of the decorated foam systems is comparable with the plain (non-decorated) foams as indicated by the dynamic viscosity measurements (see Figure 58 (a)).

For the hydrocolloid-based complementary phase, xanthan gum (XG) is used aiming to reduce the amount of organic species used in the synthesis of $\mathrm{Ag}-\mathrm{TiO}_{2}$ structures, when compared to the oil-based formulations. This formulation uses the same $\mathrm{TALH}: \mathrm{TiO}_{2}$ suspension as base, but instead of dispersing the Ag precursor in an oil-phase, it is mixed in the PAA-XG solution and incorporated to the titania mixture. Since very small amounts of $X G$ are required to significantly increase the viscosity of aqueous solutions[272-274], a more efficient transformation of $\mathrm{TALH}$ into $\mathrm{TiO}_{2}$ and the nucleation of the decorative secondary phase $(\mathrm{Ag}(0))$ could be expected upon energy treatments such as UV or thermalannealing. For this formulation, the Ag precursor was first solubilized in ethanol. A minimum amount of ammonium hydroxide was used to ensure its solvation. This solution was completely clear before mixing with the XG-PAA solution, upon mixing with such solution it became slightly turbid which is indicative of sedimentation / reduction of Ag in similar solutions[19]. Particularly, this reaction is characteristic of metallic Ag nucleation from a Tollens' reagent in contact with a polysaccharide compound like xanthan gum[275]. Then, the solution was immediately added to the $\mathrm{TiO}_{2}-\mathrm{TALH}$ suspension and frothed as customary. All the processing steps were performed in the dark to avoid light-induced uncontrolled changes of the ink.
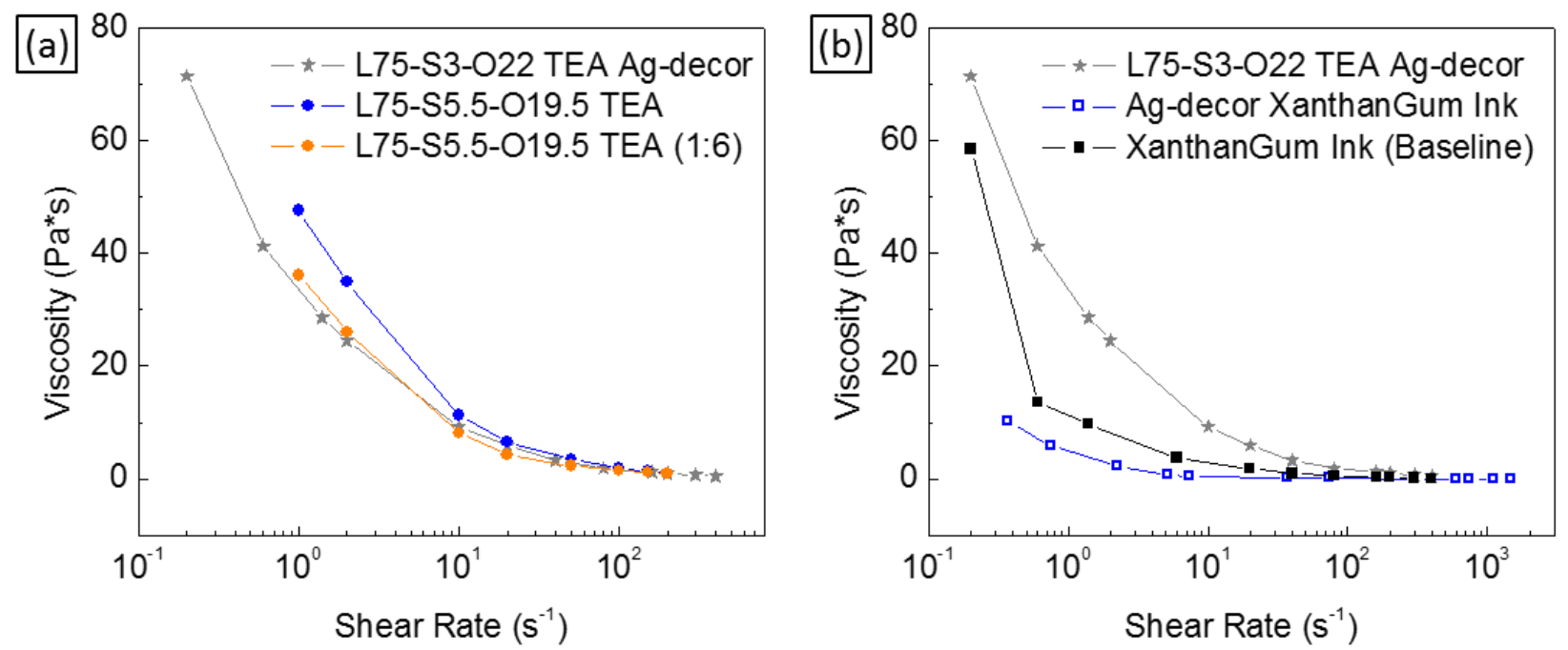

Figure 58. Viscosity of (a) representative TEA (no-Ag •) and Ag-decorated $\mathrm{TiO}_{2}$ ( $\star$ ) oil-based foam-ink; and (b) Agdecorated $\mathrm{TiO}_{2}(\star)$ oil-based foam-ink, Xanthan Gum baseline ( $(\mathbf{})$, and Xanthan Gum-based Ag-decorated $\mathrm{TiO}_{2}(\square)$ precursor inks. 
Characterization of the viscosity of the XG-baseline (without Ag), and Ag-decorated XG formulation is presented in Figure 58 (b). It can be observed that the obtained viscosity for the xanthan gum based formulations is significantly lower than that for the oil based foams. This in turn, results in the destabilization of the foam as it is sheared during the application to the substrates using doctor blading.

\subsection{Microstructure}

The difference in the morphology of the Ag-decorated composites (oil-based) and (XG-based) is apparent from the SEM and optical microscope images, where the foams stabilized using hydrocolloids rapidly allowed the solvent (water) to evaporate, resulting in the collapse of the gas macropores and the rearrangement of the structure as films. See Figure 59, Figure 60 and Figure 61.

Ambient light exposure of the films results in their staining, indicative of reactive systems. The dried films are observed to be more stable to light exposure (against staining), than when exposed while wet; with the oil based foam system exhibiting the highest stability as observed from the optical microscope images, see Figure 59. Such staining is observed to be blocked at the films' surface, as the collapsed pores of the XG-based $\mathrm{Ag}-\mathrm{TiO}_{2}$ non-sheared sample do not exhibit such staining, having collapsed while in the dark, after initial sample surface exposure to light (Figure 59 (c)). This drying/light exposure dynamics may be further investigated for their use in controlled photoreduction processes as drying causes the exposure of inner regions of the pores. This could be viewed as a potential mechanism to control "healing" of the film cracks as drying progresses, and its detailed study is left as future work.
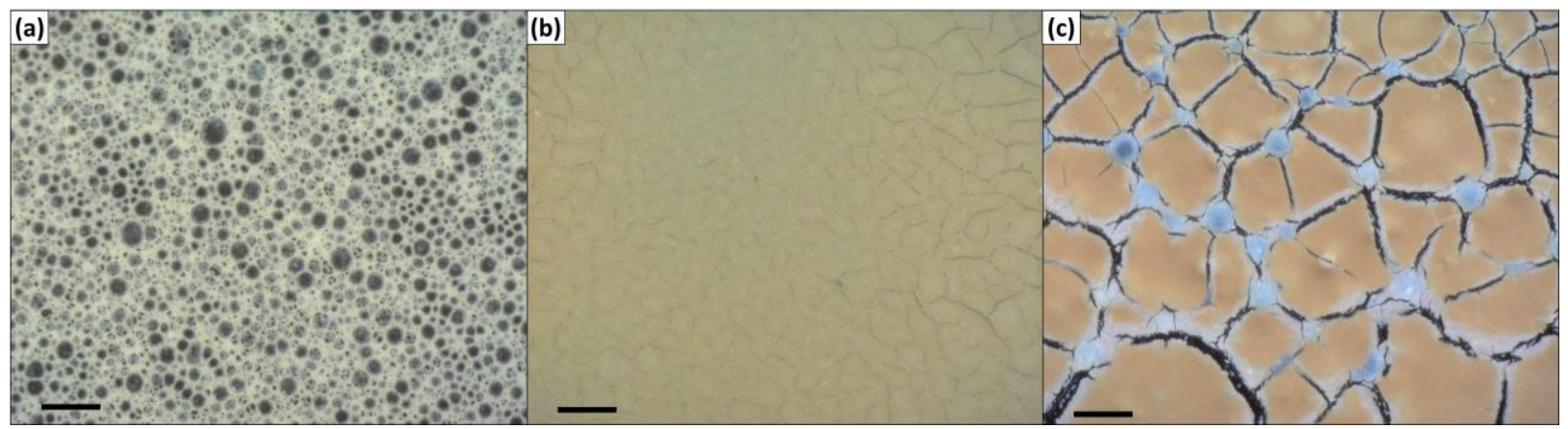

Figure 59. Optical microscope images of the Ag-decorated $\mathrm{TiO}_{2}$ samples from (a) oil-based, and xanthan gum-based formulations (b) and (c). Scale bars are $200 \mu \mathrm{m}$ long. Samples (a) and (b) were doctor bladed; sample (c) as scooped and allowed to relax as it dried.

SEM images of the Ag-decorated $\mathrm{TiO}_{2}$ foams are shown in Figure 60. The broad porosity size distribution is apparent, featuring macropores as large as $80 \mu \mathrm{m}$ and smaller features of $\sim 5 \mu \mathrm{m}$ (from trapped gas). Additionally, meso- and micro-porous structures resulting from the aggregation of the primary particles and their micro-porous features are observed. From these images, the role of the oily-scaffold is once more elucidated - see Figure 60 (high-magnification of "As Doctor Bladed" sample) - which shows that the oil phase serves as scaffold structure for the assembly of the titania suspension, maintaining the macropore structure as the solvent is evaporated during drying of the films. 


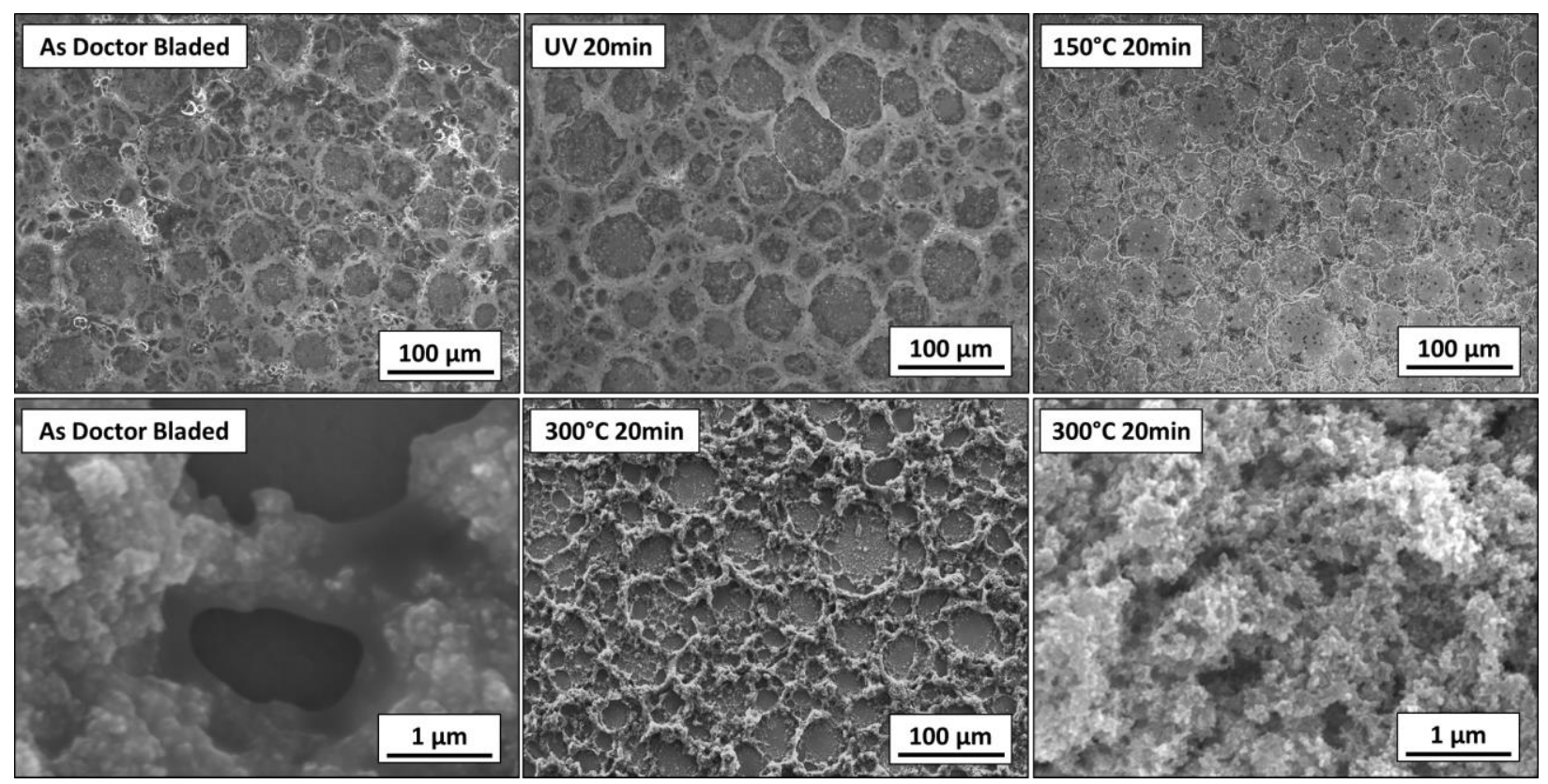

Figure 60. SEM images of the L75-S3-O22 Ag-decorated $\mathrm{TiO}_{2}$ foam composites treated at different temperature and UV light $(\lambda=254 \mathrm{~nm})$ conditions for $20 \mathrm{~min}$.

For the XG-based $\mathrm{Ag}_{-} \mathrm{TiO}_{2}$ film, mud-cracking is observed (see Figure 61), forming as the solvent evacuation progresses, due to relatively high localized tensile stresses [246]. At this point, it becomes necessary to highlight the structural role of the oil phase which prevents the structure from cracking upon drying. However, during sintering, its cracking behavior will depend on additional aspects such as open- or closedcell configurations, and relative L:S:O and $\mathrm{TALH}: \mathrm{TiO}_{2}$ ratios[276]; as discussed in the previous chapter.
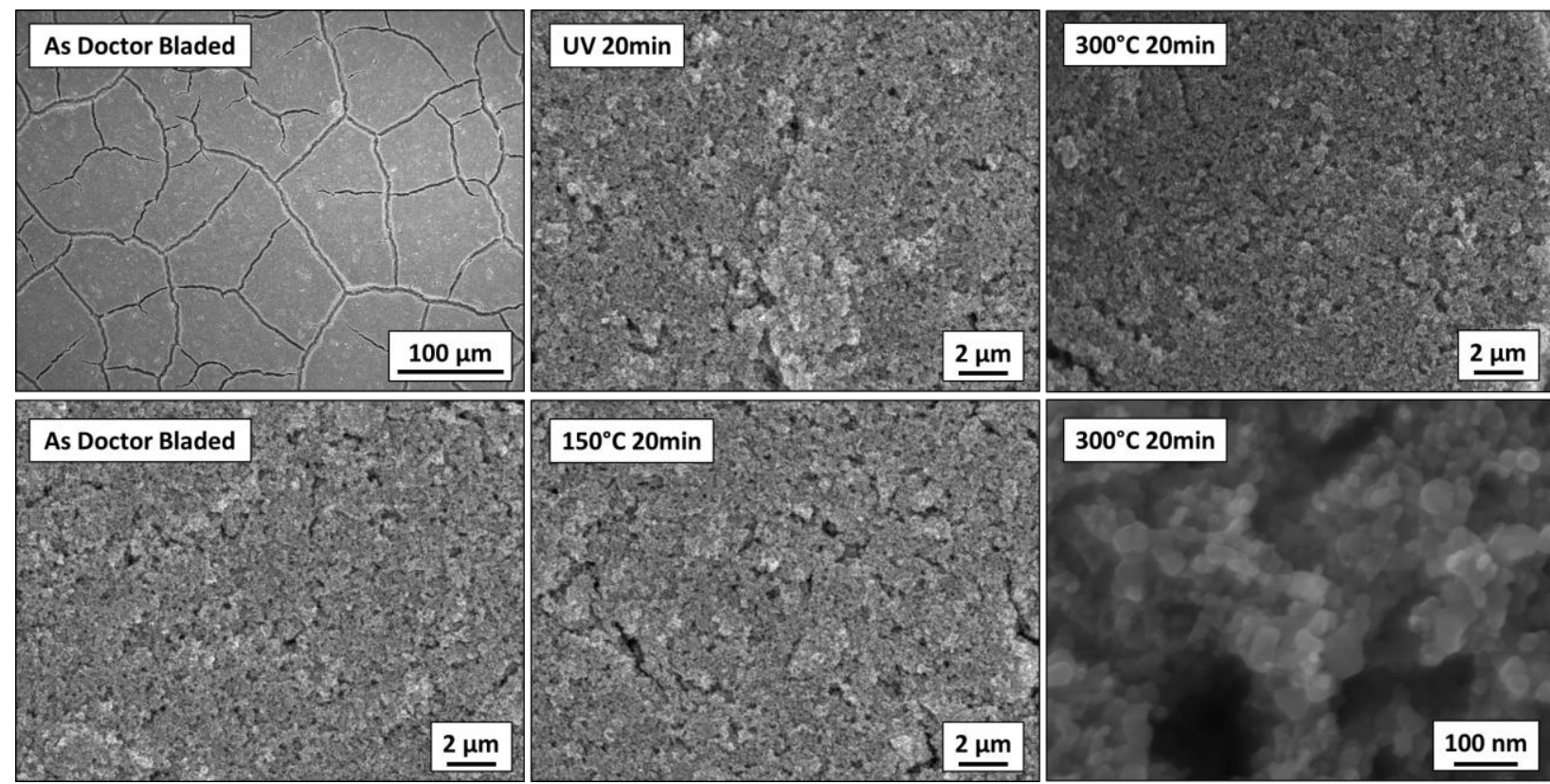

\section{$300^{\circ} \mathrm{C} 20 \mathrm{~min}$}

Figure 61. SEM images of the Xanthan gum-based Ag-decorated $\mathrm{TiO}_{2}$ composites treated at different temperature and UV light $(\lambda=254 \mathrm{~nm})$ conditions for $20 \mathrm{~min}$. 
Both XG-stabilized inks, (Ag-decorated and baseline) result in similar film morphologies as observed from Figure 61 and Figure 62. The particle aggregation and mud cracking observed is characteristic of the $\mathrm{TALH}: \mathrm{TiO}_{2}$ ink systems as depicted in chapter 5 .

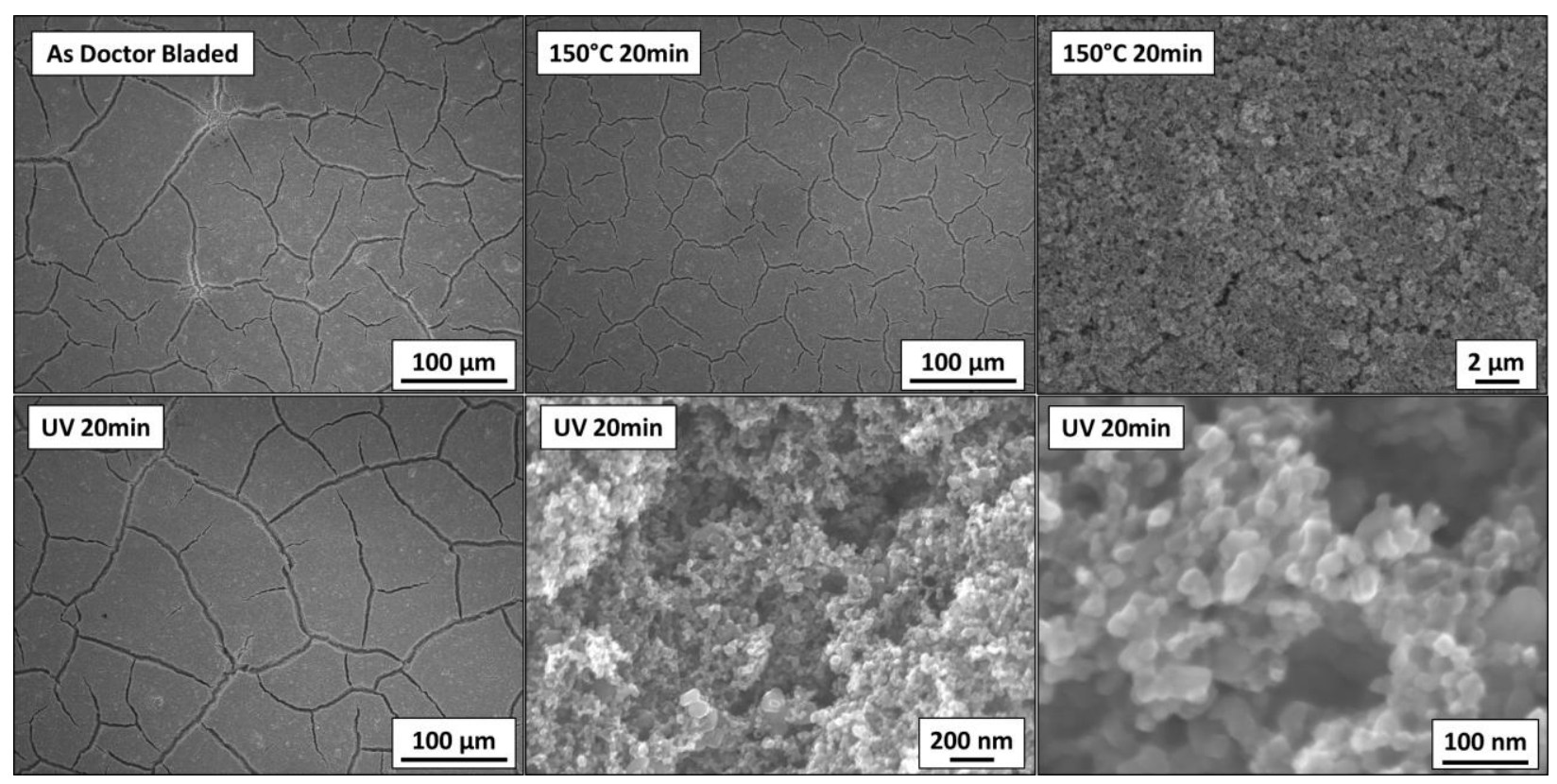

Figure 62. SEM images of the Xanthan gum-based $\mathrm{TiO}_{2}$ baseline composites, treated at $150^{\circ} \mathrm{C}$ and $\mathrm{UV}$-light $(\lambda=254$ nm) for $20 \mathrm{~min}$.

XRD patterns for the Ag-decorated $\mathrm{TiO}_{2}$ oil-based foam (see Figure 63), exhibit rutile and anatase phases as expected from the primary $\mathrm{TiO}_{2}$ particles. The signal intensity is observed to be relatively similar to that of the ITO substrate, and is associated to the thin and porous character of the fabricated films, so that the collected signal resulted mainly from the substrate. Furthermore, from the XRD patterns, no clear evidence of $\mathrm{Ag}$ in metallic phase is obtained, which is mainly due to the low amount of $\mathrm{Ag}$ used for the decoration of the $\mathrm{TiO}_{2}$ surfaces, $\sim 1.2 \mathrm{wt} \%$ ( $\sim 0.3$ atomic\%) of the total sintered composite (no remaining organic compounds); and to the small size of the formed Ag nanoparticles, with characteristic peaks significantly broader and lower in intensity when compared to those of $\mathrm{TiO}_{2}$. Therefore, the peaks from metallic Ag may be confused with the background signal. Additionally, the diffraction peak with $100 \%$ relative intensity for metallic $\mathrm{Ag}$ should be located at $\sim 38.5^{\circ} 2 \theta$ angle, which may coincide with those for anatase at $\sim 38.01^{\circ}$ and $38.84^{\circ}$ (with expected larger intensity from the higher $\mathrm{TiO}_{2}$ content), see Figure 63 (b). 

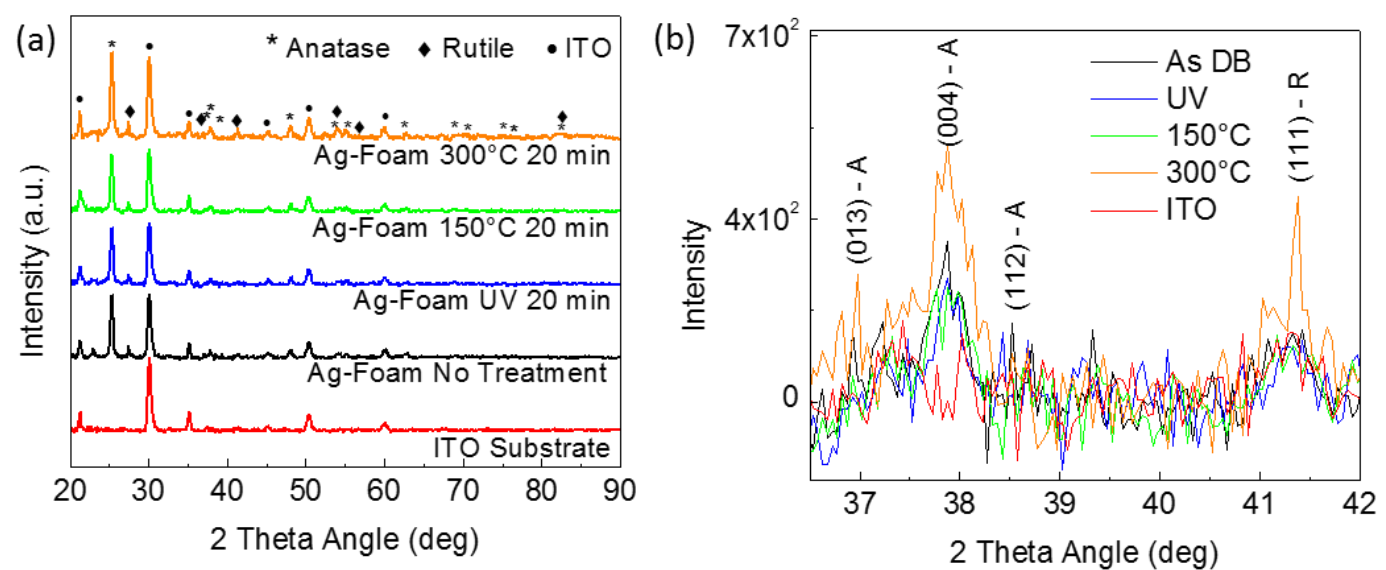

Figure 63. XRD patterns of the L75-S3-O22 Ag-TiO 2 foam composites treated at different temperature and UV light conditions. (b) Detailed XRD regions where the metallic Ag peak would show.

The XRD patterns for the XG-based inks (see Figure 64 (a) and (b)) show significantly higher intensity for the $\mathrm{TiO}_{2}$ compared to the substrate signal, which is attributed to thicker films and more coverage of the substrate surface as can be inferred from the respective SEM images. Nevertheless, similarly to the Ag$\mathrm{TiO}_{2}$ oil-based foam, no conclusive evidence of $\mathrm{Ag}$ in metallic phase is observed from XRD, see Figure 64 (c) and (d). The Ag content is kept identical for both oil-based and XG-based $\mathrm{Ag}-\mathrm{TiO}_{2}$ composites.
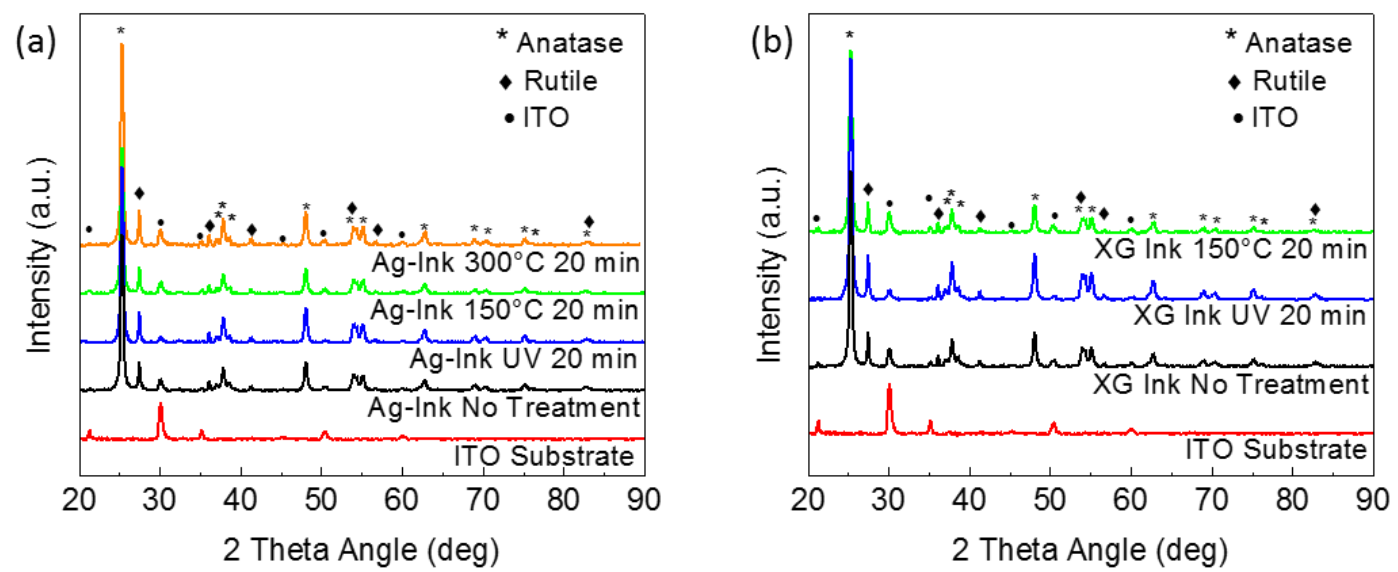

(c)

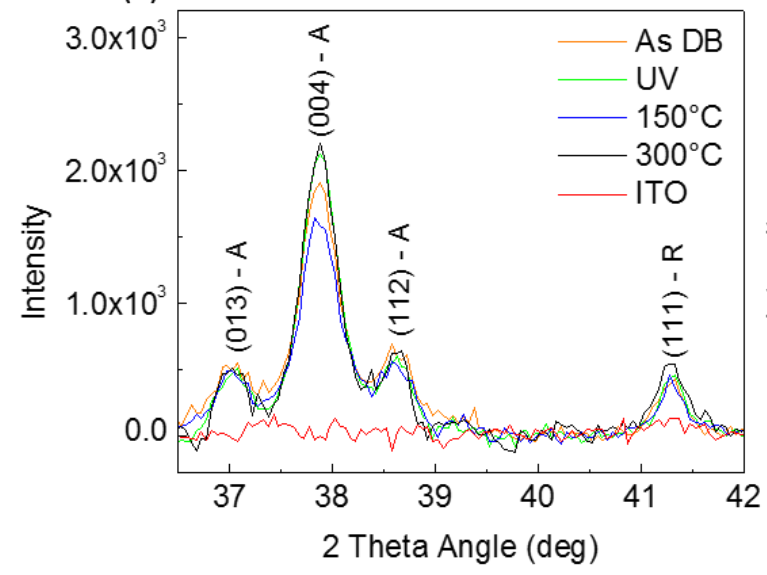

(d)

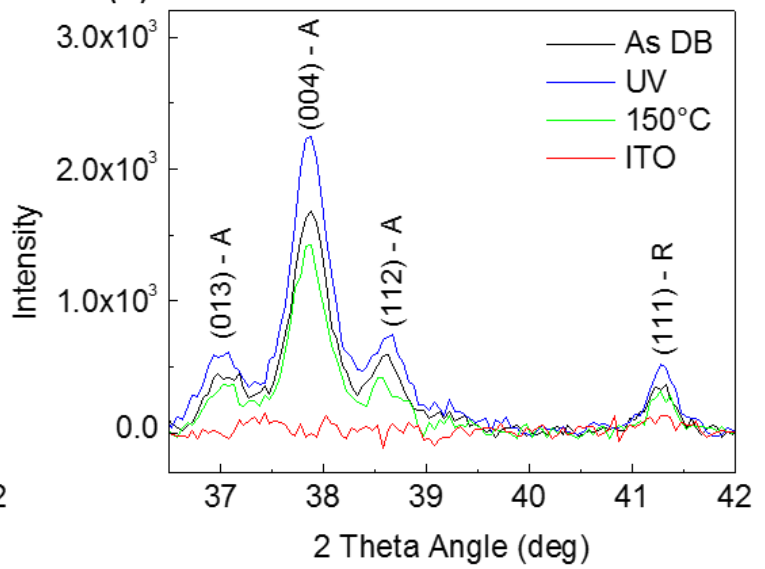

Figure 64. XRD patterns of the xanthan gum based $\mathrm{TiO}_{2}$ inks ( $a$ and $c$ ) Ag-decorated and ( $b$ and $d$ ) without Ag (baseline xanthan gum- $\mathrm{TiO}_{2}$ ). (c and d) Detailed XRD regions where the metallic Ag peak would show. 
EDS information for the $\mathrm{Ag}-\mathrm{TiO}_{2}$ composite systems were collected - see Appendix $\mathrm{A}$ - and show gradual removal of the organic species as more energy is supplied in the post-processing treatments. Table 21 summarizes the different $\mathrm{C}: \mathrm{Ti}$ and $\mathrm{Ag}: \mathrm{Ti}$ atomic \% ratios for the samples treated under such conditions. It is observed that almost no signal from Ag is obtained for the oil based samples, and may be explained by the small volume of sample (thin and porous); and the large amount of organics present, hindering the detection of $\mathrm{Ag}$ (in minimum concentration compared to the other elements detected). Nevertheless, $\mathrm{Ag}$ is detected for the $300^{\circ} \mathrm{C}-20 \mathrm{~min}$ treatment, which significantly removes the organic species associated to the oil phase of the foam. The latter is evident from the C:Ti ratio, that decreases as more energy is provided. In contrast, the lower organics content in the XG Ag-decorated $\mathrm{TiO}_{2}$ composites shows readily the $\mathrm{Ag}$ content even for the lower energy treatments, and exhibits a less pronounced decrease of the $\mathrm{C}: \mathrm{Ti}$ ratio.

Table 21. C:Ti and Ag:Ti atomic \% ratios from the EDS spectra for the different energy treated $\mathrm{Ag}_{\text {-decorated } \mathrm{TiO}_{2}}$ composites.

\begin{tabular}{|c|c|c|c|}
\hline EDS - Films & Treatment & C:Ti Ratio & Ag:Ti Ratio \\
\hline Oil-based & As Doctor Bladed & 5.840 & 0.000 \\
\hline \multirow[t]{3}{*}{$\mathrm{Ag}-\mathrm{TiO}_{2}$ Foam } & 20min UV & 6.503 & 0.000 \\
\hline & $150^{\circ} \mathrm{C} 20 \mathrm{~min}$ & 7.537 & 0.000 \\
\hline & $300^{\circ} \mathrm{C} 20 \mathrm{~min}$ & 1.828 & 0.010 \\
\hline XG-based & As Doctor Bladed & 0.192 & 0.010 \\
\hline \multirow[t]{3}{*}{$\mathrm{Ag}-\mathrm{TiO}_{2} \operatorname{lnk}$} & 20min UV & 0.147 & 0.016 \\
\hline & $150^{\circ} \mathrm{C} 20 \mathrm{~min}$ & 0.145 & 0.019 \\
\hline & $300^{\circ} \mathrm{C} 20 \mathrm{~min}$ & 0.175 & 0.010 \\
\hline
\end{tabular}

EDS mapping of the XG- and oil-based Ag-TiO 2 composites (see Figure 65 and Figure 66), show uniform distribution of the $\mathrm{Ag}$ on the $\mathrm{TiO}_{2}$, since no particularly high contrast spots of $\mathrm{Ag}$ are identified. The spectroscopic information from the mapped regions is labelled as "Selected Area" and is depicted in the magenta box for the XG-based $\mathrm{Ag}-\mathrm{TiO}_{2}$ composite (Figure 66). The selected area for the oil-based sample constitutes the entire imaged area in Figure 65. By design, the target compounds should only consist of $\mathrm{Ti}, \mathrm{O}$ and $\mathrm{Ag}$ species. However, the utilization of organic precursor compounds and their incomplete removal is responsible for the $\mathrm{C}$ and $\mathrm{N}$ content, while all other species information originates from the substrate (ITO on glass). 


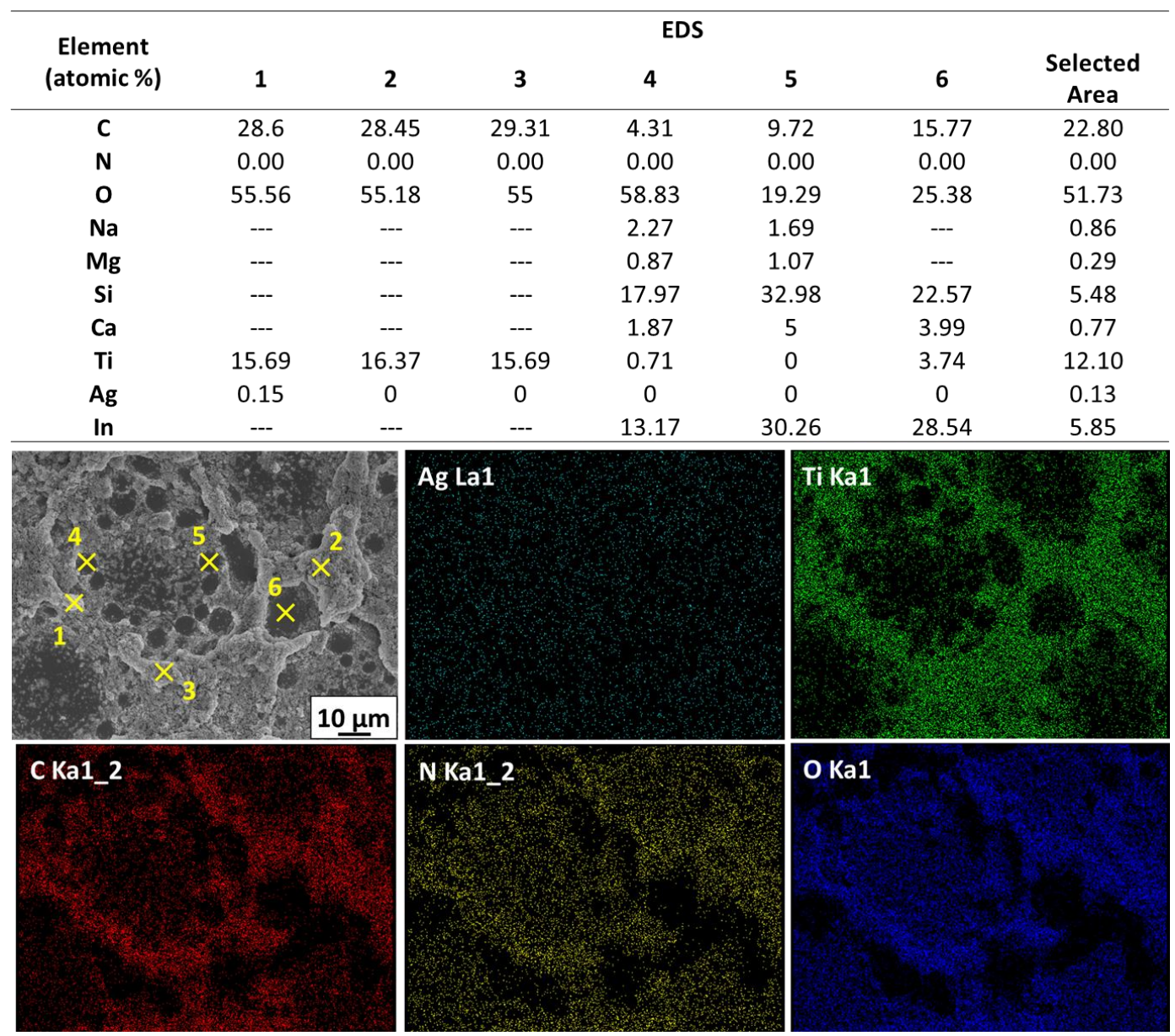

Figure 65. EDS point analysis and mapping of the oil-based Ag-decorated $\mathrm{TiO}_{2}$ foam treated at $300^{\circ} \mathrm{C}$ for $20 \mathrm{~min}$.

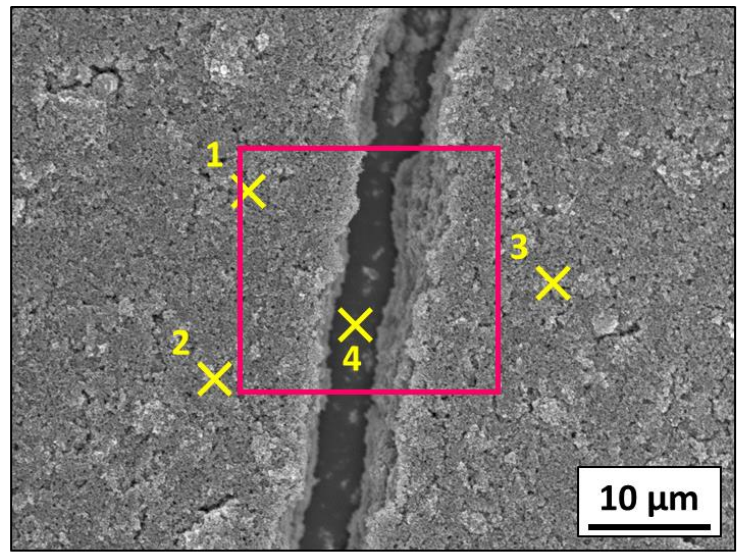

\begin{tabular}{cccccc}
\hline $\begin{array}{c}\text { Element } \\
\text { (atomic } \\
\text { \%) }\end{array}$ & $\mathbf{1}$ & $\mathbf{2}$ & $\mathbf{3}$ & $\mathbf{4}$ & $\begin{array}{c}\text { EDS } \\
\text { Selected } \\
\text { Area }\end{array}$ \\
\hline $\mathrm{C}$ & 4.73 & 4.62 & 5.69 & --- & --- \\
$\mathrm{N}$ & 0.00 & 0.00 & 0.00 & 0.00 & 0.00 \\
$\mathrm{O}$ & 54.14 & 67.32 & 57.35 & 47.21 & 66.41 \\
$\mathrm{Si}$ & --- & --- & --- & 0.47 & 1.55 \\
$\mathrm{~K}$ & --- & --- & --- & --- & 2.16 \\
$\mathrm{Ca}$ & --- & --- & --- & 3.66 & --- \\
$\mathrm{Ti}$ & 40.58 & 27.63 & 36.33 & 23.89 & 29.88 \\
$\mathrm{Ag}$ & 0.55 & 0.44 & 0.64 & --- & --- \\
$\mathrm{In}$ & --- & --- & --- & 24.77 & --- \\
\hline
\end{tabular}
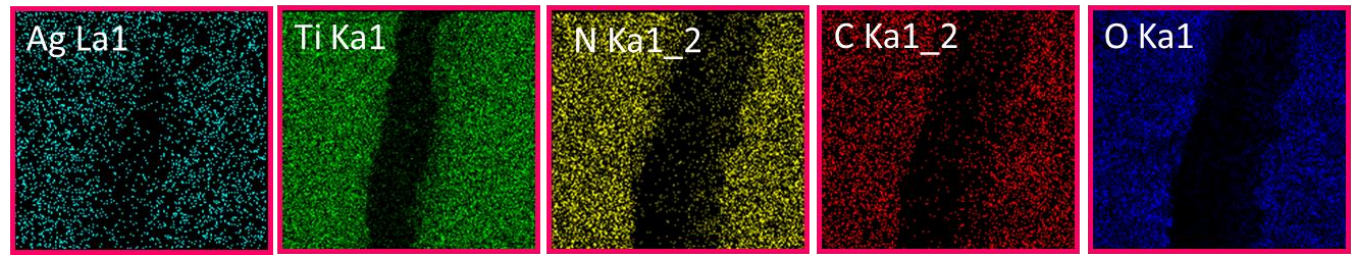

Figure 66. EDS point analysis and mapping of the xanthan gum-based Ag-decorated $\mathrm{TiO}_{2}$ composite treated with UVlight $(\lambda=254 \mathrm{~nm})$ for $20 \mathrm{~min}$. 


\subsection{Opto-electronic Properties and Microstructure}

Despite the challenges encountered to obtain quantitative information about the $\mathrm{Ag}$ decorations from EDS and XRD (especially for the oil-based foam), the $\mathrm{Ag}-\mathrm{TiO}_{2}$ composites exhibit interesting coloration changes suggesting the presence of nanoparticles on the surface of $\mathrm{TiO}_{2}$ [277] as is characteristic from the plasmonic effects, see Figure 67.

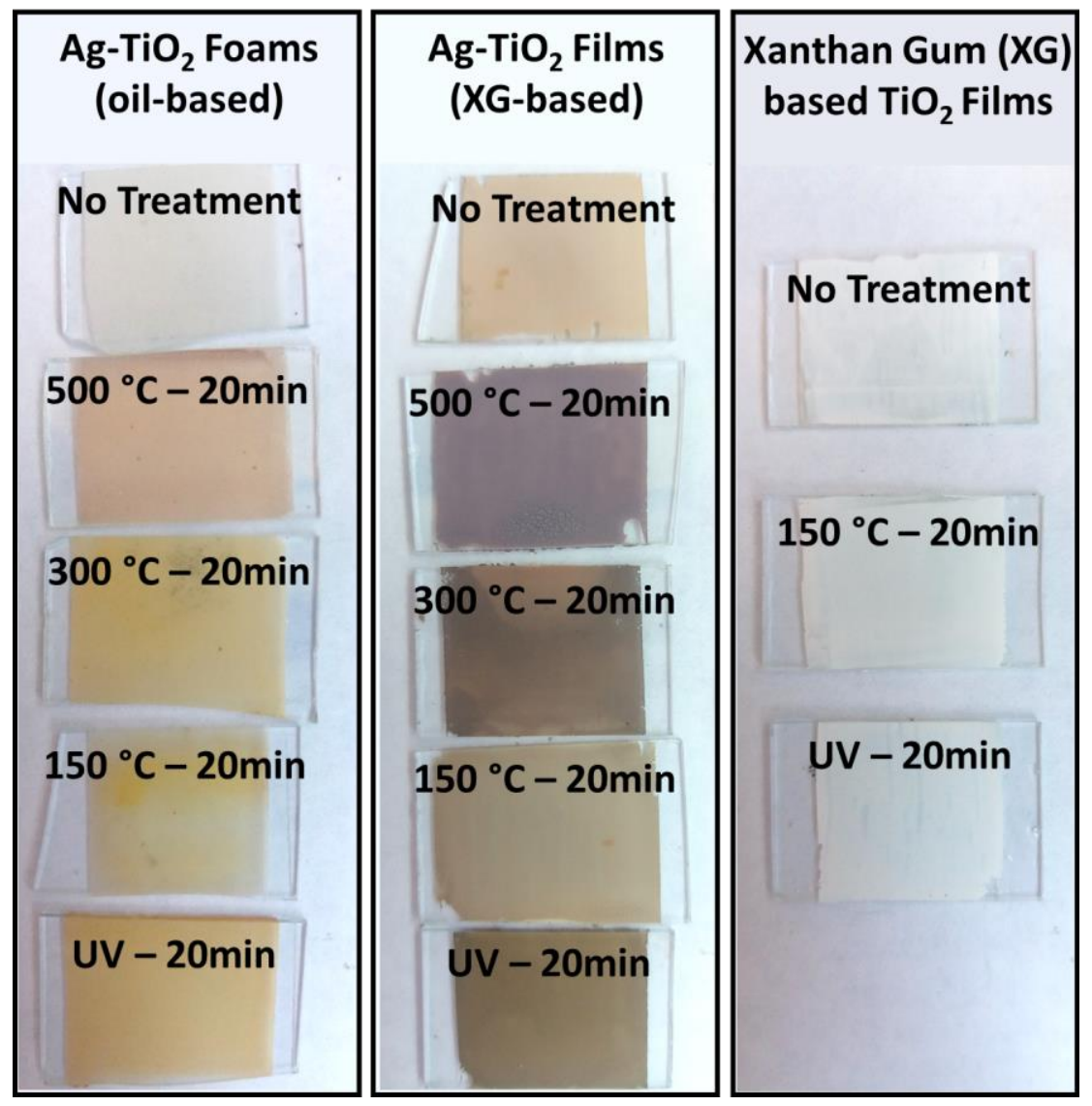

Figure 67. Photograph of the doctor bladed $\mathrm{Ag}$-decorated $\mathrm{TiO}_{2}$ samples treated under different energy conditions.

The optical bandgap of the studied samples, as calculated using the Tauc's relationship (eq. 5)[278], is observed to vary slightly according to the energy treatment administered, see Table 22 . All the obtained values are characteristic of $\mathrm{TiO}_{2}$ (3.1 eV - rutile; $3.3 \mathrm{eV}$ anatase)[18]. Tauc plots and UV-Vis spectra of the different $\mathrm{Ag}-\mathrm{TiO}_{2}$ oil-based and $\mathrm{XG}$-based $\mathrm{TiO}_{2}$ films are presented in Figure 68 and Figure 69, respectively. For the oil-based $\mathrm{Ag}-\mathrm{TiO}_{2}$ foams, a general increase in the $\mathrm{E}_{\mathrm{g}}$ values is observed with increasing energy of the treatments. The lower energy treatments (i.e. UV and $150^{\circ} \mathrm{C}$, for $20 \mathrm{~min}$ ) exhibit $\mathrm{E}_{\mathrm{g}}$ values closer to that of rutile $\mathrm{TiO}_{2}$, and as the treatment temperature is increased, the obtained $\mathrm{E}_{\mathrm{g}}$ values increase gradually to values close to that for anatase $\mathrm{TiO}_{2}$. On the other hand, for the XG-based Ag-decorated $\mathrm{TiO}_{2}$ films, the $E_{g}$ values are observed to decrease systematically as the post-deposition energy treatments is increased. Also, the values for the plain (no $\mathrm{Ag}$ ) $\mathrm{TiO}_{2} \mathrm{XG}$-based films show $\mathrm{E}_{\mathrm{g}}$ values with no significant variation. 
Table 22. Optical band gap $\left(E_{g}\right)$ of Ag-decorated $\mathrm{TiO}_{2}$ composites, and conduction band edge $\left(E_{c b}\right)$ of decorating $\mathrm{Ag}$ particles. ${ }^{*}$ Calculated from Tauc plots (linear region extrapolation). ${ }^{* *}$ Calculated using the Einstein's photon energy equation.

\begin{tabular}{|c|c|c|c|}
\hline Sample & $\mathrm{E}_{\mathrm{g}}(\mathrm{eV})^{*}$ & $\lambda_{\max }$ & $\mathrm{E}_{\mathrm{cb}}(\mathrm{eV})^{* *}$ \\
\hline Ag- $\mathrm{TiO}_{2}$ Foam As Doctor Bladed & 3.2000 & 453 & 2.7373 \\
\hline $\mathrm{Ag}-\mathrm{TiO}_{2}$ Foam UV 20min & 3.1205 & 440 & 2.8214 \\
\hline $\mathrm{Ag}-\mathrm{TiO}_{2}$ Foam $150^{\circ} \mathrm{C} 20 \mathrm{~min}$ & 3.1719 & 432 & 2.8704 \\
\hline $\mathrm{Ag}-\mathrm{TiO}_{2}$ Foam $300^{\circ} \mathrm{C} 20 \mathrm{~min}$ & 3.2103 & 437 & 2.8375 \\
\hline $\mathrm{Ag}-\mathrm{TiO}_{2}$ Foam $500^{\circ} \mathrm{C} 20 \mathrm{~min}$ & 3.2793 & 491 & 2.5255 \\
\hline $\mathrm{Ag}-\mathrm{TiO}_{2}$ XG-Film As Doctor Bladed & 3.2760 & 456 & 2.7193 \\
\hline $\mathrm{Ag}-\mathrm{TiO}_{2}$ XG-Film UV 20min & 3.2603 & 440 & 2.8182 \\
\hline $\mathrm{Ag}-\mathrm{TiO}_{2} \mathrm{XG}-\mathrm{Film} 150^{\circ} \mathrm{C} 20 \mathrm{~min}$ & 3.2323 & 440 & 2.8182 \\
\hline $\mathrm{Ag}-\mathrm{TiO}_{2}$ XG-Film $300^{\circ} \mathrm{C} 20 \mathrm{~min}$ & 3.1290 & 469 & 2.6439 \\
\hline $\mathrm{Ag}-\mathrm{TiO}_{2} \mathrm{XG}-\mathrm{Film} 500^{\circ} \mathrm{C} 20 \mathrm{~min}$ & 3.1229 & 523 & 2.3709 \\
\hline XG-based $\mathrm{TiO}_{2}$ Film As Doctor Bladed & 3.2521 & --- & --- \\
\hline XG-based $\mathrm{TiO}_{2}$ Film UV 20min & 3.2662 & --- & --- \\
\hline XG-based $\mathrm{TiO}_{2}$ Film $150^{\circ} \mathrm{C} 20 \mathrm{~min}$ & 3.2790 & --- & --- \\
\hline
\end{tabular}

In the Tauc plots (Figure 68 (a) and Figure 69 (a) and (c)), the intercept of the line fitting the linear region of the end tail of the $(\alpha h v)^{2}$ vs. $h v$ with the abscissa[278], is used to determine the bandgap of the prepared samples.

$$
(\alpha h v)^{2}=A\left(h v-E_{g}\right)^{n}
$$

Where $n=1 / 2$ for direct band gap semiconductors, $A$ is a constant, $h v$ is the photon energy, and $\alpha$ is the absorption coefficient of the semiconductor. The latter in turn, can be calculated from Eq. 6, where $\mathrm{k}$ is the absorbance as measured using UV-Vis spectroscopy.

$$
\alpha=\frac{4 \pi k}{\lambda}
$$

The conduction band energy $\left(E_{c b}\right)$ of the decorating $A g$ nanoparticles has been calculated based on the UV-Vis information using the Einstein photon energy equation (Eq. 7) and is also included in Table 22.

$$
E_{c b}=\frac{h c}{\lambda_{\max }}
$$

Where $h$ is the Plank constant, $c$ is the speed of light, and $\lambda_{\max }$ the wavelength of maximum absorbance for the Ag peaks form the UV-Vis spectra. Our results for the $E_{c b}$ of these nanoparticles are comparable with those obtained for spherical Ag nanoparticles of similar diameter[26]. The Ag nanoparticles sizes for the representative $\mathrm{Ag}-\mathrm{TiO}_{2}$ composite systems has been determined from TEM as is discussed later in this chapter. 

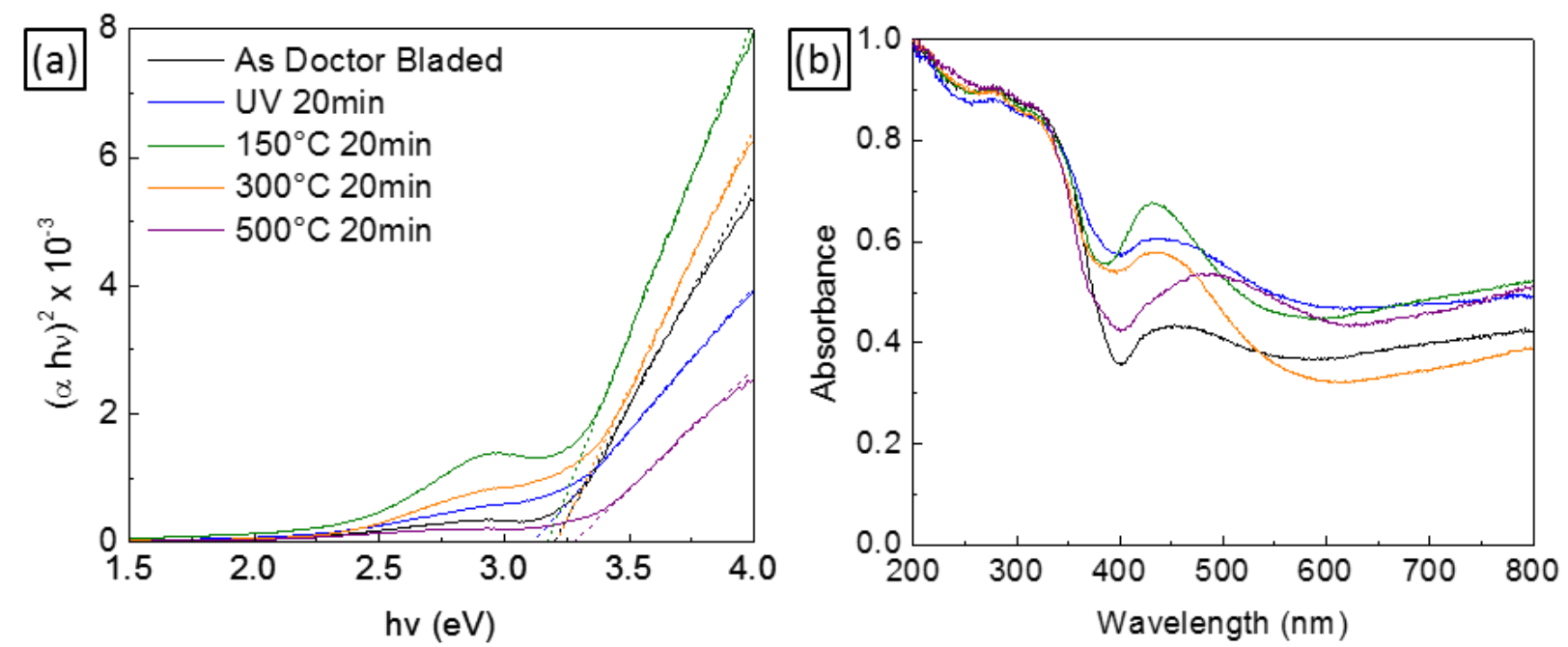

Figure 68. (a) Tauc plots for the determination of the optical bandgap, (b) normalized UV-Vis spectra of the differently treated oil-based $\mathrm{Ag}-\mathrm{TiO}_{2}$ foams.
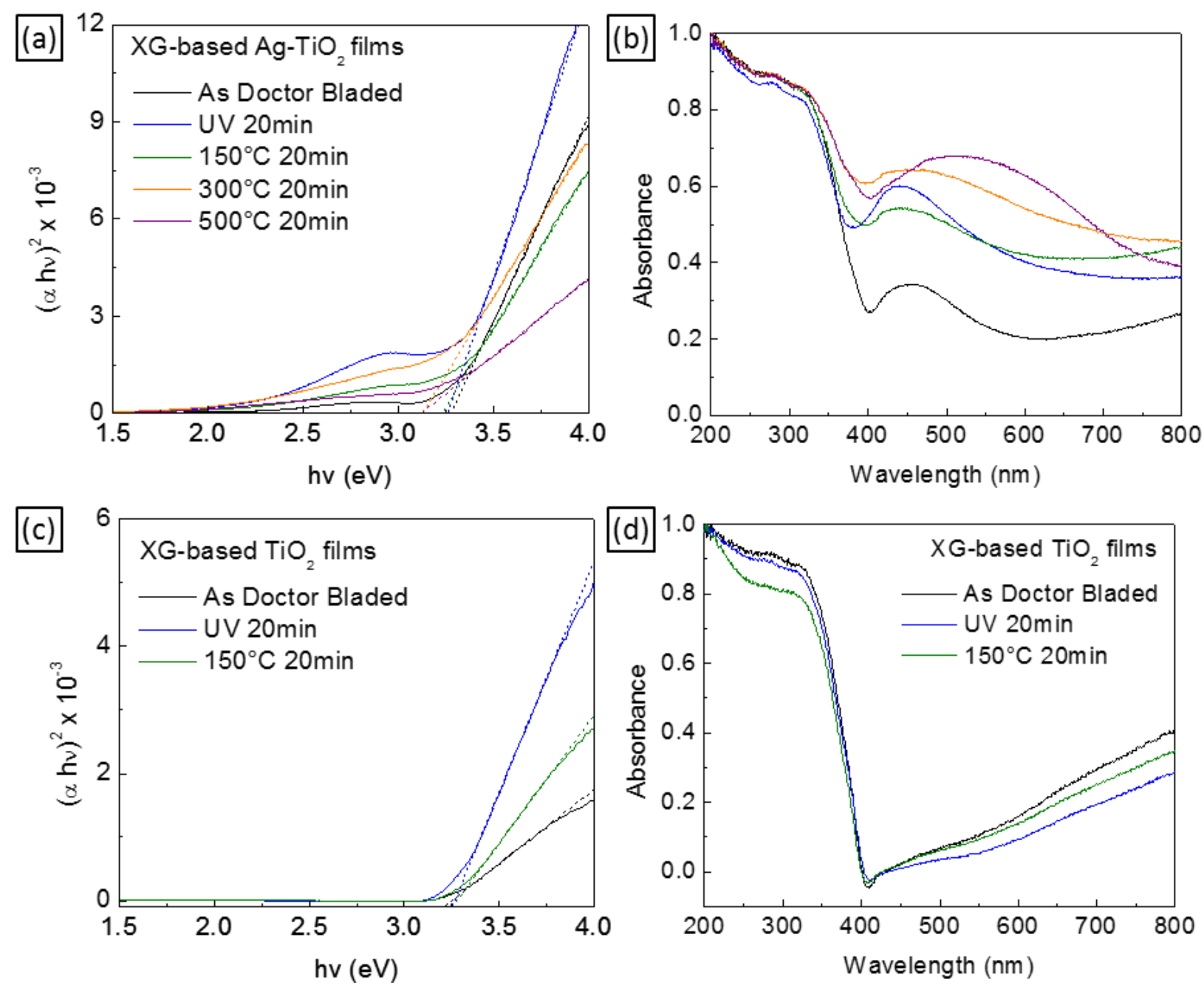

Figure 69.Tauc plots for the determination of the optical bandgap (a) - XG-based Ag-TiO films, (c) - XG-based TiO films; and normalized UV-Vis spectra (b) - XG-based Ag-TiO 2 films, (d) - XG-based $\mathrm{TiO}_{2}$ films, treated under different energy conditions. 
It is therefore of great interest to understand the nature of such changes in the $E_{g}$, since they may originate from localized effects of the $\mathrm{Ag}$ nanoparticles on the $\mathrm{TiO}_{2}$ surface (also known as band edge bending)[279]; as well as from the effect of the $\mathrm{Ag}$ nanoparticles on the crystallization of $\mathrm{TiO}_{2}$ favoring the rutile phase formation at remarkably low temperatures $[280,281]$. In these multiphase systems, where some of the $\mathrm{TiO}_{2}$ is crystallizing from $\mathrm{TAHL}$, the Ag nanoparticles may induce such crystallization to take the rutile phase instead of the anatase polymorph, as has been reported for $\mathrm{TiO}_{2}$-Ag nanocomposites prepared from $\mathrm{TiCl}_{4}$ using photodeposition and annealed at $600^{\circ} \mathrm{C}$ [280]. Typically, for the temperature ranges explored in this work (up to $500^{\circ} \mathrm{C}$ ), the crystallization of $\mathrm{TALH}$ into $\mathrm{TiO}_{2}$ from suspension formulations without $\mathrm{Ag}$, occurs in the anatase phase[128]. However, the influence of the Ag nanoparticles (nucleating and growing in the proposed composite systems), may cause a deviation of such behavior. Such influence is expected to be significantly higher when using the $\mathrm{XG}$-based $\mathrm{Ag}-\mathrm{TiO}_{2}$ formulations, than when using the oil-based $\mathrm{Ag}-\mathrm{TiO}_{2}$ route. The reason being, that in the oil-based $\mathrm{Ag}-\mathrm{TiO}_{2}$ foams, the nucleation and growth of the $\mathrm{Ag}$ nanoparticles is expected to be generally smaller than in the oil-free XG systems, for equivalent energy treatments, due to the lower viscosity of the XG-based formulations. Also, since less energy is required to decompose the added XG, than the oil phase used as gas stabilizer of the $\mathrm{TiO}_{2}$-TALH aqueous suspension, the available energy is expected to nucleate the Ag nanoparticles more efficiently in the XG case. Additionally, since the $\mathrm{Ag}$ precursor is solubilized in an aqueous solution (for the $\mathrm{XG}$ case), the $\mathrm{TiO}_{2}$ surfaces are readily available for the $\mathrm{Ag}$ ions to nucleate. In contrast, during the synthesis of the $\mathrm{Ag}-\mathrm{TiO}_{2}$ foams from the oil-based formulations, a significant amount of energy is used for the removal of the organics composing such phase (in which the Ag-precursor is solubilized). Therefore, for the $\mathrm{Ag}$ nanoparticles to induce the anatase to rutile phase transition, such particles would need to reach the sites where TALH is transforming. For such system (oil-based), the possible Ag nucleation sites are within the oil phase (where enough $\mathrm{Ag}^{+}$ions cluster), and/or at the boundaries between the oil phase and the aqueous titania suspension. The latter being the preferred case as $\mathrm{TiO}_{2}$ surfaces in the suspension would provide ordered nucleation sites, as well as photoreduction centers[282] leading to Ag nuclei, in the case of the samples subjected to UV light treatments.

In order to confirm the nucleation of $\mathrm{Ag}$ nanoparticles from the investigated systems, representative samples, with presumably larger Ag nanoparticles were imaged using TEM. Figure 70 shows images of the Ag-decorated $\mathrm{TiO}_{2}$ foams from the oil-based system treated at $500^{\circ} \mathrm{C}(\mathrm{a})$ and (b); and from the xanthan gum-based system, treated at $300^{\circ} \mathrm{C}$ (c)-(e), and $500^{\circ} \mathrm{C}$ (f) and (g). TEM imaging of the primary $\mathrm{TiO}_{2}$ nanoparticles was also performed for comparison purposes, see Figure 71.

In the TEM images of the $\mathrm{Ag}-\mathrm{TiO}_{2}$ composites, large $\mathrm{TiO}_{2}$ formations can be distinguished. Scattered around such formations, smaller Ag nanoparticles are observed as highlighted with the dashed ovals and arrows. In contrast, the images taken from the primary $\mathrm{TiO}_{2}$ particles, exhibit overall smaller and more uniform sized $\mathrm{TiO}_{2}$ particles that aggregate forming clusters. The difference in particle aggregation for the latter, contrasting the $\mathrm{Ag}-\mathrm{TiO}_{2}$ systems can clearly be observed from Figure 70 (b), (d) and (g), where the large $\mathrm{TiO}_{2}$ formations show necking between neighboring $\mathrm{TiO}_{2}$ particles; whereas Figure 71 (b) shows overlapping of primary $\mathrm{TiO}_{2}$ particles. Additionally, the oil-based and $\mathrm{XG}$-based $\mathrm{Ag}-\mathrm{TiO}_{2}$ composites show differences in the sample morphology at the nanoscale. For the oil-based system, a more continuous $\mathrm{TiO}_{2}$ structure is observed when compared to the XG-based sample structures, regardless of the temperature conditions utilized. The latter result highlights the advantages of using immiscible phases for the 
dispersion of the decorating material precursor as in the case of the oil-based system, ensuring high connectivity of the metal-oxide scaffold.

The formed Ag particles, exhibit spherical shape with diameter sizes ranging from $\sim 2-4 \mathrm{~nm}$ for the $500^{\circ} \mathrm{C}$ treated oil-based $\mathrm{Ag}-\mathrm{TiO}_{2}$ foams; and from $\sim 2-3 \mathrm{~nm}$ and $\sim 3-10 \mathrm{~nm}$, for the XG-based Ag-TiO composites treated at $300^{\circ} \mathrm{C}$ and $500^{\circ} \mathrm{C}$, respectively. Inset (e) shows one of the spherical Ag nanoparticles on the $\mathrm{TiO}_{2}$ surface. Since the nucleated $\mathrm{Ag}$ particles are observed to grow when increasing the energy treatment from 300 to $500^{\circ} \mathrm{C}$ (for the XG-based $\mathrm{Ag}-\mathrm{TiO}_{2}$ composites), it may be inferred that the size of the nucleated $\mathrm{Ag}$ particles for the samples treated using lower energy conditions exhibit smaller sizes.

The coloration of the samples, is indicative of different nanoparticle sizes. Also, the $E_{c b}$ of metal nanoparticles is dependent on their size[24-26]; thus, a correlation between the Ag-decorating nanofeatures and the calculated $\mathrm{E}_{\mathrm{cb}}$ can be established. 


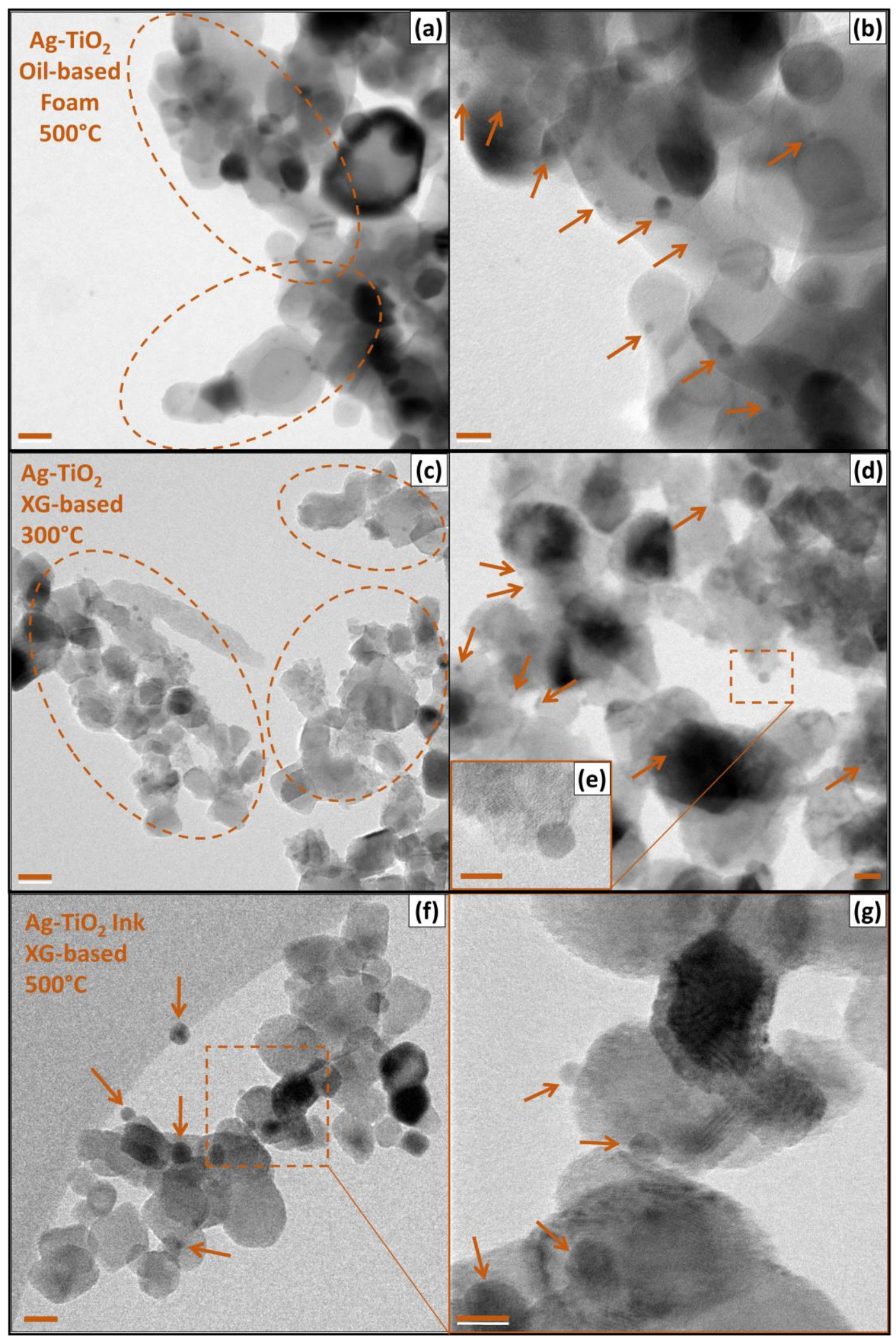

Figure 70. TEM images of the oil-based $500^{\circ} \mathrm{C}$ treated $\mathrm{Ag}-\mathrm{TiO}_{2}$ foams (a) and (b); and xanthan gum-based $\mathrm{Ag}-\mathrm{TiO}_{2}$ composites treated at $300^{\circ} \mathrm{C}(\mathrm{c}-\mathrm{e})$, and $500^{\circ} \mathrm{C}(\mathrm{f})$ and $(\mathrm{g})$. Scale bars are $20 \mathrm{~nm}$ for (a), (c) and (f); $10 \mathrm{~nm}$ for (b), (d) and (g); and $5 \mathrm{~nm}$ for (e). Arrows indicate some of the Ag nanoparticles. Dashed ovals depict Ag nanoparticle rich areas. Dashed squares indicate enlarged regions in $(e)$, and $(g))$. 


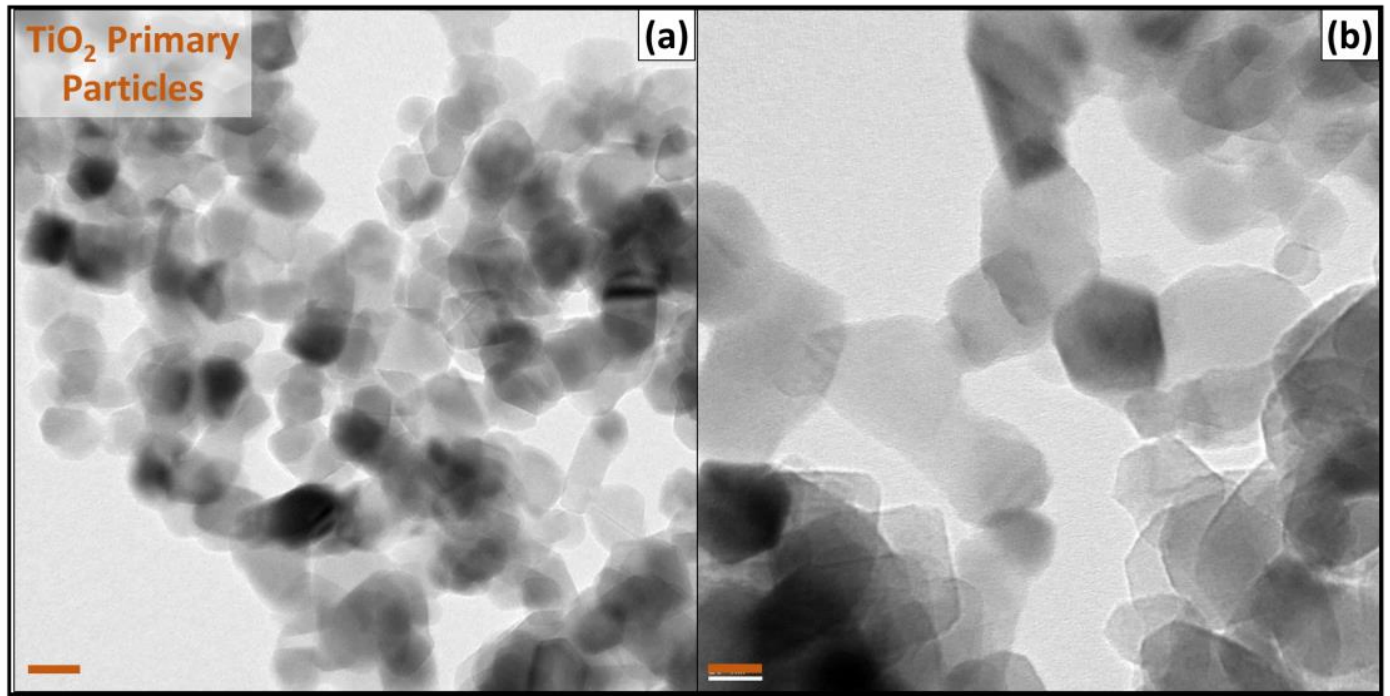

Figure 71. TEM images of the primary $\mathrm{TiO}_{2}$ particles. Scale bars in (a) and (b) correspond to 20 and $10 \mathrm{~nm}$ respectively.

\subsection{Chemical State of Nucleated Secondary-Phase Nanoparticles}

X-ray photoelectron spectroscopy (XPS) was used to investigate the oxidation state of the nucleated Ag nanoparticles and confirm their metallic state, as well as to assess the transformation of the samples with the respective energy treatments. For the oil-based $\mathrm{Ag}$-decorated $\mathrm{TiO}_{2}$ foam composites, the intensity of the Ag3d, Ti2p and O1s peaks is observed to increase as more energy is supplied, see Figure 72. In contrast, the intensity of the $\mathrm{C} 1 \mathrm{~s}$ peak decreases as expected from the organics decomposition with increasing energy. Accordingly, the $\mathrm{O}$ band at $\sim 530 \mathrm{eV}$, corresponding to the Ti-O bond, is observed to increase at the expense of the $\mathrm{O}$ band at $\sim 532.5 \mathrm{eV}$, characteristic of the $\mathrm{C}-\mathrm{O}$ bond. 

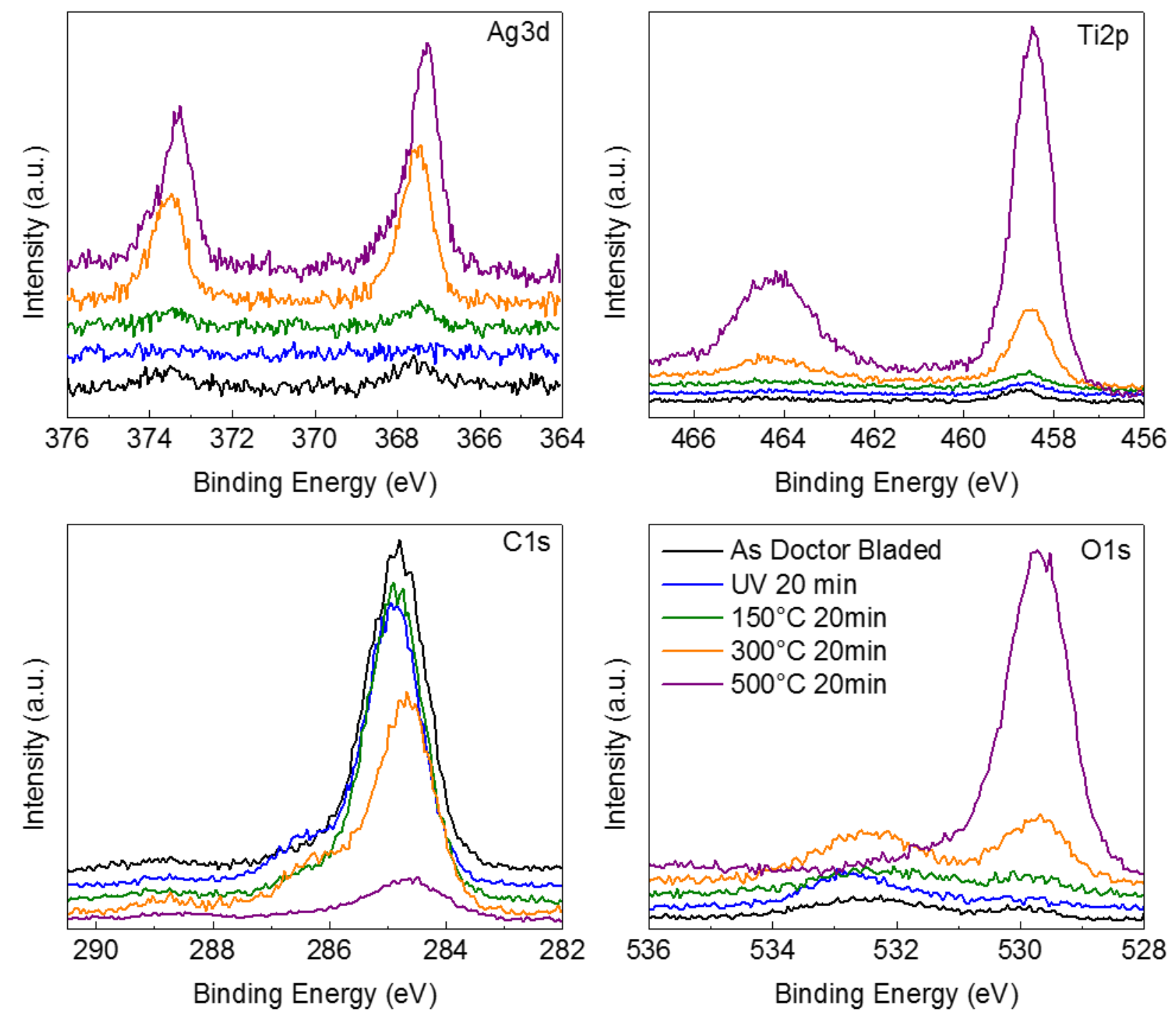

Figure 72. X-ray photoelectron spectroscopy detailed peaks Ag 3d, Ti 2p, C 1s and O 1s, for the oil-based Ag-decorated $\mathrm{TiO}_{2}$ foam composites.

The XPS data collected for the XG-based Ag-decorated $\mathrm{TiO}_{2}$ composites displays a general decrease of the C1s peak, which is associated to the organics removal with increasing energy (see Figure 73). No significant peak shape change is observed for the $\mathrm{O} 1 \mathrm{~s}$ and $\mathrm{C} 1 \mathrm{~s}$ peaks, indicative of the reduced amount of organics in the XG-based formulation, when compared to the oil-based foams. However a slight shift is observed for the $500^{\circ} \mathrm{C}$ treated samples, which is more pronounced for the Ti2p and O1s peaks with a $\sim 0.4 \mathrm{eV}$ difference. This shift may be partially attributed to error in the calibration, since it is also observed in the C1s peak centered at $284.9 \mathrm{eV}$ - displaced $\sim 0.15 \mathrm{eV}$ compared to the other samples centered $\sim 284.7$ $284.8 \mathrm{eV}$ - in turn coinciding with that obtained for $\mathrm{TiO}_{2}$ primary particles, see Appendix A. Additionally, diminishing of the shoulder at $\sim 531.5 \mathrm{eV}$ for the $01 \mathrm{~s}$ peak can be observed as the temperature is increased. The intensity of the $\mathrm{Ag} 3 \mathrm{~d}$ peak for the $500^{\circ} \mathrm{C}$ treated specimen, is significantly higher than that for the other samples; whereas the intensity for all the other peaks, is observed to be similar regardless of the energy treatment employed. These results agree with those from XRD and EDS, exhibiting stronger 
signal from the inorganic species for the XG thickened composites when compared to the oil-based counterparts.
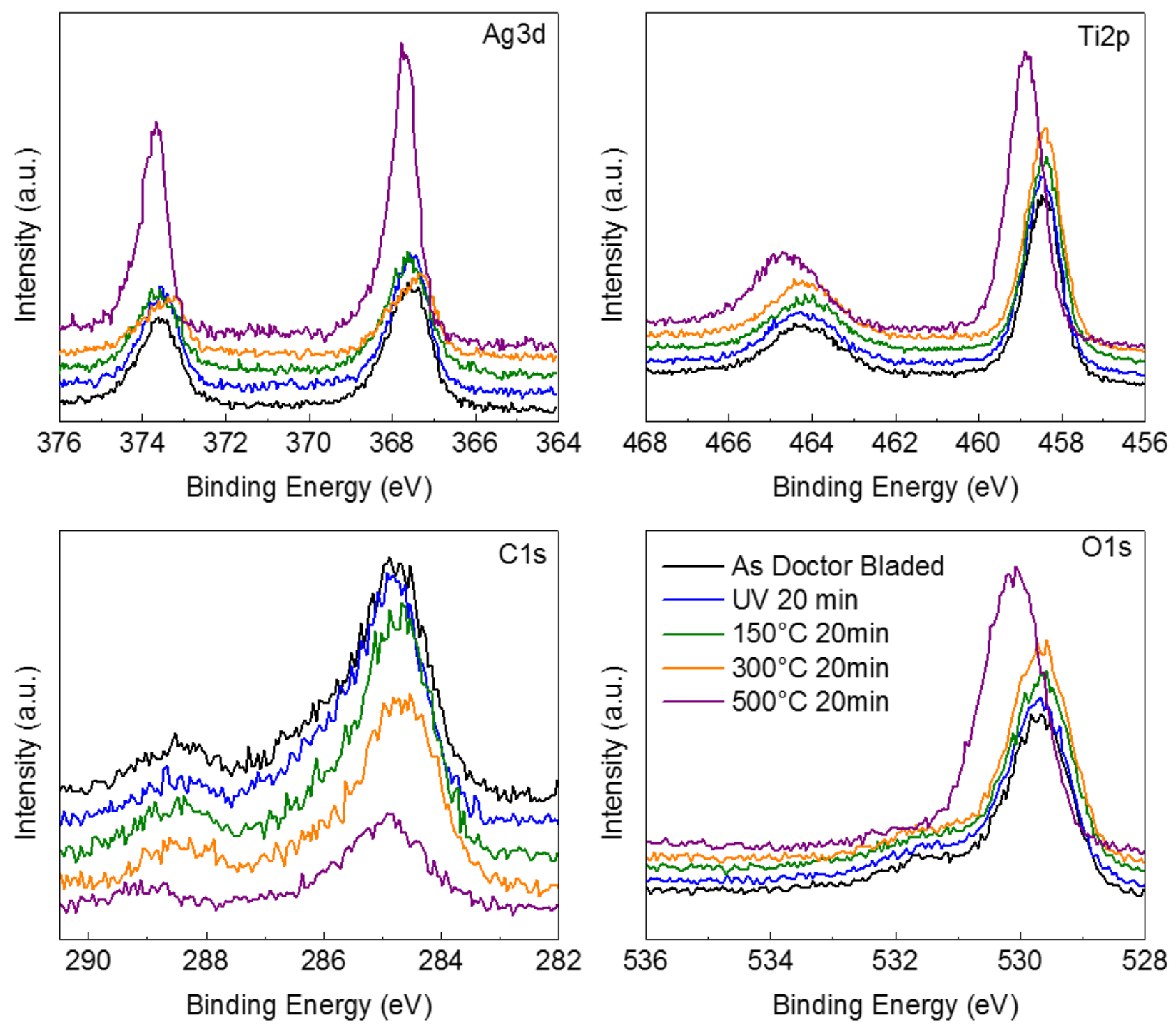

Figure 73. X-ray photoelectron spectroscopy detailed peaks Ag 3d, Ti 2p, C 1s and O 1s, for the xanthan gum-based Ag-decorated $\mathrm{TiO}_{2}$ ink composites.

Due to the incomplete removal of the organic species from the foams/inks studied when using low energy treatments (UV and $150^{\circ} \mathrm{C}$ ), it may be argued that the nucleated secondary phase materials correspond to mixtures of the $\mathrm{Ag}(0)$ with $\mathrm{AgO}, \mathrm{Ag}_{2} \mathrm{O}$; and possibly $\mathrm{Ag}_{2} \mathrm{CO}_{3}$. Calculation of the modified Auger parameters (AP)[283-285] from the XPS data, indicate values $726 \mathrm{eV}$ (see Table 23), which are characteristic of the $\mathrm{Ag}(0)$, i.e. metallic $\mathrm{Ag}[286,287]$. Additionally, the non-significant change observed in the binding energy for the $A g 3 \mathrm{~d}_{5 / 2}$ peak among different energy treatments, it can be taken as strong suggestion of the metallic character of the particles forming within the system. 
Table 23. X-ray photoelectron spectroscopy binding energy and modified Auger parameters AP for the Ag3d $5 / 2$ peak from the Ag-decorated $\mathrm{TiO}_{2}$ composites.

\begin{tabular}{|c|c|c|c|c|c|}
\hline Sample & Treatment & $\begin{array}{c}\text { Binding } \\
\text { Energy } \\
\text { Ag3d }_{5 / 2}(\mathrm{eV})\end{array}$ & $\begin{array}{c}\text { Kinetic Energy } \\
\mathrm{Ag} \mathrm{M}_{4} \mathrm{~N}_{45} \mathrm{~N}_{45} \\
(\mathrm{eV})\end{array}$ & $\begin{array}{c}A P-3 d_{5 / 2} \\
M_{4} N_{45} N_{45}(e V)\end{array}$ & $\begin{array}{c}A P-3 d_{5 / 2} \\
M_{5} N_{45} N_{45}(e V)\end{array}$ \\
\hline Oil-based & As Doctor bladed & 367.60 & 358.60 & 726.20 & 719.20 \\
\hline $\mathrm{Ag}-\mathrm{TiO}_{2}$ & UV 20min & 367.54 & 358.60 & 726.14 & 719.14 \\
\hline \multirow[t]{3}{*}{ foams } & $150^{\circ} \mathrm{C} 20 \mathrm{~min}$ & 367.44 & 358.60 & 726.04 & 719.04 \\
\hline & $300^{\circ} \mathrm{C} 20 \mathrm{~min}$ & 367.52 & 358.60 & 726.12 & 719.12 \\
\hline & $500^{\circ} \mathrm{C} 20 \mathrm{~min}$ & 367.27 & 359.30 & 726.57 & 723.87 \\
\hline \multirow[t]{2}{*}{ XG-based } & As Doctor bladed & 367.62 & 358.60 & 726.22 & 719.22 \\
\hline & UV 20min & 367.52 & 359.30 & 726.82 & 721.32 \\
\hline \multirow[t]{3}{*}{${\mathrm{Ag}-\mathrm{TiO}_{2} \text { films }}$} & $150^{\circ} \mathrm{C} 20 \mathrm{~min}$ & 367.62 & 358.80 & 726.42 & 719.92 \\
\hline & $300^{\circ} \mathrm{C} 20 \mathrm{~min}$ & 367.37 & 359.30 & 726.67 & 719.67 \\
\hline & $500^{\circ} \mathrm{C} 20 \mathrm{~min}$ & 367.77 & 358.80 & 726.57 & 721.57 \\
\hline
\end{tabular}

Quantitative information on the XPS composition of the investigated samples is included in the Table 24. The O:Ti ratio obtained is in all cases higher than 2 (the stoichiometric value for $\mathrm{TiO}_{2}$ ); this ratio is observed to decrease as more energy is supplied to the oil-based $\mathrm{Ag}-\mathrm{TiO}_{2}$ foam systems, whereas it is kept relatively constant for the XG-based $\mathrm{Ag}-\mathrm{TiO}_{2}$ films. The $\mathrm{Ag}$ content is generally found to be higher than that measured from EDS (see Table 21). Because of the higher vacuum conditions met by the XPS equipment compared to the vacuum in the EDS instrument, the XPS values could be considered more accurate.

Table 24. Quantitative analysis of the sample composition, as calculated from the XPS peak fittings.

\begin{tabular}{|c|c|c|c|c|c|c|c|c|}
\hline \multirow[b]{2}{*}{ Ink } & \multirow[b]{2}{*}{ Treatment } & \multicolumn{6}{|c|}{ Atomic \% } & \multirow[b]{2}{*}{ Ag : Ti Ratic } \\
\hline & & C & 0 & $\mathrm{Ti}$ & $\mathrm{Ag}$ & $\mathbf{N}$ & O : Ti Ratio & \\
\hline \multicolumn{2}{|c|}{$\mathrm{TiO}_{2}$ Aeroxide (Primary Particles) } & 30.43 & 51.06 & 18.51 & --- & --- & 2.76 & --- \\
\hline MTDF-03 & As Doctor bladed & 91.13 & 6.56 & 0.90 & 0.11 & 1.30 & 7.29 & 0.12 \\
\hline Oil-based & UV 20min & 85.92 & 11.50 & 0.75 & 0.07 & 1.75 & 15.33 & 0.09 \\
\hline Ag- & $150^{\circ} \mathrm{C} 20 \mathrm{~min}$ & 86.27 & 10.81 & 1.64 & 0.07 & 1.20 & 6.59 & 0.04 \\
\hline decorated & $300^{\circ} \mathrm{C} 20 \mathrm{~min}$ & 68.32 & 25.94 & 4.84 & 0.37 & 0.52 & 5.36 & 0.08 \\
\hline $\mathrm{TiO}_{2}$ foam & $500^{\circ} \mathrm{C} 20 \mathrm{~min}$ & 16.96 & 59.20 & 23.04 & 0.63 & 0.18 & 2.57 & 0.03 \\
\hline MTDF-04 & As Doctor bladed & 28.74 & 51.26 & 17.90 & 1.14 & 0.97 & 2.86 & 0.06 \\
\hline Xanthan & UV 20min & 29.24 & 49.55 & 18.55 & 1.19 & 1.46 & 2.67 & 0.06 \\
\hline gum-based & $150^{\circ} \mathrm{C} 20 \mathrm{~min}$ & 28.23 & 51.12 & 18.41 & 1.04 & 1.20 & 2.78 & 0.06 \\
\hline $\mathrm{Ag}-$ & $300^{\circ} \mathrm{C} 20 \mathrm{~min}$ & 23.75 & 52.72 & 21.78 & 0.76 & 0.99 & 2.42 & 0.03 \\
\hline decorated & $500^{\circ} \mathrm{C} 20 \mathrm{~min}$ & 11.10 & 61.20 & 25.02 & 1.87 & 0.81 & 2.45 & 0.07 \\
\hline
\end{tabular}

XPS survey and detailed scans for the different $\mathrm{Ag}-\mathrm{TiO}_{2}$ systems and the primary $\mathrm{TiO}_{2}$ particles are included in Appendix A. 


\subsection{Concluding Remarks}

Using the proposed and investigated multiphase emulsion material systems, secondary phase functionalizing features can be produced within the emulsion system, by dispersing/encapsulating their precursors in a complimentary phase of the emulsion and inducing their nucleation using energy treatments such as UV-light exposure or sintering. This method enables a novel, sustainable and relatively simple route, for the fabrication of hierarchically ordered cellular mesoporous ceramics with embedded functional nanofeatures. A distinction between the oil-based $\mathrm{Ag}-\mathrm{TiO}_{2}$ foams and the xanthan gum-based $\mathrm{Ag}-\mathrm{TiO}_{2}$ composites should be made, since the latter do not yield cellular macropore structures for the XG concentrations used in this investigation. The xanthan gum concentrations utilized are relatively low thus not providing enough stabilization for the macropores (i.e. gas bubbles), observed to collapse upon drying of the film and/or application of shearing stress. Characterization of the $\mathrm{Ag}-\mathrm{TiO}_{2}$ composites subjected to the different energy treatments was successfully done through XRD, EDS, SEM, XPS, UV-Vis spectroscopy and TEM. The nucleated nanoparticles are found to be in the metallic state as highlighted from the XPS results, and exhibit spherical shape with sizes below $10 \mathrm{~nm}$. The distribution of the $\mathrm{Ag}$ nanoparticles is observed to be rather uniform on the $\mathrm{TiO}_{2}$ surface. This investigation using $\mathrm{Ag}$ on $\mathrm{TiO}_{2}$ structures, provides an alternative approach for the nucleation of secondary phase materials on metal-oxide structures with controllable microstructures, useful across multiple applications from energy to biomedical, including $\mathrm{H}_{2}$ production though enhanced light-harvesting devices, photovoltaics, small molecule detection, catalyst, water cleaning and regeneration systems, and bio-compatible materials. 


\section{Conclusions}

Through the investigation of the different ink systems for additive manufacturing, important relationships were established regarding their design, processing, and materials properties. These relationships are expected to be useful for the synthesis of similar ink-material systems and their implementation in devices, using CDW and alternative printing techniques.

Relevant aspects addressed in the processing include the stabilization and rheology control of colloidal ink systems; and the consideration of the inks' $\mathrm{pK}_{\alpha}$ properties influencing the processing requirements and resulting materials microstructure.

The understanding of the interactions of the different material precursors and processing conditions involved in the synthesis of $\mathrm{Ag}$ nanoparticles from the investigated solution system, serves as the basis for the design of processing protocols for their sustainable production.

Similarly, the influence of the printing parameters on the materials morphology and crystalline orientation of Al-doped $\mathrm{ZnO}$ structures has been investigated through microstructural evolution studies, suggesting additional routes for their control.

The studies of hybrid $\mathrm{TiO}_{2}$-based inks consisting of inorganic primary particles and organic metal-oxide precursors, contribute to the understanding of fundamental aspects and issues of hybrid inks design, printing and processing. This investigation provided the preliminary information necessary for the implementation of such hybrid titania systems in more complex ink formulations, that allowed for their 3D printing, and morphology tuning as open- or closed-cell cellular mesoporous structures, and controlling of their surface area properties. Moreover, such investigations (TALH:TiO ${ }_{2}$ system and $\mathrm{Ag}$ nucleation) allow the realization of composite metal/metal-oxide systems through the incorporation of secondary phase decorative materials and/or their precursors by their encapsulation in one of the phases of such multi-phase systems.

The use of hydrocolloids (i.e. methyl-cellulose and xanthan gum) as rheology enhancers and colloidal stabilization agents, was implemented for the $\mathrm{Ag}$ and $\mathrm{TiO}_{2}$ based systems. XG in particular showed significant increase in viscosity with minimum amounts shows promise towards reducing the thermal budget associated to these materials curing/sintering.

Finally, assessment of the ink designs and processing methods from a sustainable perspective has been performed. This assessment may be considered as a pioneering approach towards important paradigm changes in ink development; and, constitute efforts for bridging the gap between lab practice and industrially adopted synthesis methods. The central issue being the inherent materials and precursors innocuous character, which makes them safe (throughout their life) and highly compatible for biomedical applications.

Some specific contributions of this work include:

a.) The investigation of the nucleation of Ag nanoparticles and the effect of the reducing agent (MEA vs. DEA) viscosity and $\mathrm{pH}$, the nucleation temperature and time, in increasing the yield of the Ag solids from $11 \%$ to $61 \%$. 
b.) The investigation of the electrical properties of the printed $\mathrm{Ag}$ patterns, processed at low temperatures (up to $150^{\circ} \mathrm{C}$ ), and the correlation of their microstructure with the electrical resistance recovery behavior upon mechanical stress release during cyclic tensile stress loading conditions. Specifically, the Ag patterns exhibit resistivity values in the order of $10^{-4} \Omega \cdot \mathrm{cm}$ when processed at $150^{\circ} \mathrm{C}$; and their microstructure, allows for the dislocation annihilation at the interfaces the Ag structures, thus preventing their failure.

c.) The study of the main factors influencing the texturing of Al-doped $\mathrm{ZnO}$ structures from sol-gel inks when using CDW, unveiling the texturing mechanism and accompanying morphological evolution. Particularly, it can be concluded that more texturing along the (0002) direction is induced due to the more efficient rearrangement of the $(10 \overline{1} 0)$ and $(10 \overline{1} 1)$ crystalline planes of the densifying film as induced by sintering when less material is printed (i.e. when tuning the printing parameters to yield finer features).

d.) The investigation of the effects of the relative amounts of Ti-organic (TALH) to $\mathrm{TiO}_{2}$ primary particles, and different polymeric agents (PAA and PVP), on the crystallization of $\mathrm{TiO}_{2}$ from $\mathrm{TALH}$ forming bridging structures that connect neighboring primary particles, using low thermal-energy treatments compatible with polymeric substrates. Indicating that 1:6 $\mathrm{TALH}: \mathrm{TiO}_{2}$ ratios maximize the crystallite growth, and that PAA further aids for the bridging of neighboring $\mathrm{TiO}_{2}$ particles and for anchoring of the inks onto the investigated substrates (Ag/PEN, ITO/PEN and Glass).

e.) The investigation of the relationships between the ink constituents, the inks' viscous properties, printing parameters, and ink/substrate interactions, that allowed progressively the transition from printing planar features, to printing 3D free-standing and spanning structures.

f.) The correlation between the compositional and processing parameters with the photocatalytic properties of the investigated TALH:TiO ${ }_{2}$ systems, which take into account the roughness and mesoporous character of the films. Moreover, it is demonstrated that the photocatalytic performance of the fabricated hierarchically ordered mesoporous cellular titania structures depends not only on the surface roughness properties, but also on the cellular architectures' configuration (i.e. open- vs. closed-cell foams). Opencellular $\mathrm{TiO}_{2}$ samples exhibit higher performance which is thought to be related to the combinatory effects of the MB solution circulation and the scattering of light towards inner regions of the cellular structure.

g.) The proposal and realization of a relatively simple one-step approach for the synthesis of 3D printable hierarchically ordered mesoporous cellular ceramics, based on environment-friendly precursors making them sustainable and industry transferable; and controlling the pore size, open- vs. closed-cell configuration and surface area properties by tuning the liquid:solid:oil and $\mathrm{TALH}: \mathrm{TiO}_{2}$ ratios.

h.) Insight on the differences from the post-processing (i.e. heat treatment vs. UV-light curing) of the investigated ceramic foams, and the mechanism of elimination of the oil phase.

i.) The proposal and demonstration of a single-step, alternative route for the synthesis of $\mathrm{Ag}$-decorated $\mathrm{TiO}_{2}$ nanocomposites, by dispersing the decorative materials and/or their precursors in one of the phases of the multiphase inks (i.e. oil- or XG-based solutions), and inducing their nucleation and growth through heat and UV-treatments. The decorating materials exhibit spherical shape and diameter ranging from 2 to 10 $\mathrm{nm}$, and consist of metallic Ag, as suggested by TEM and XPS studies. 


\section{Future Work}

The results from the investigation of the synthesis of Ag nanoparticles and the influence of the reducing agents type and concentration, and temperature-time processing relationships may be used for further improving the particle yield. Also, refining of the ink composition and the potential use of hydrocolloids other than cellulose may aid towards attaining printed features of $\sim 5-10 \mu \mathrm{m}$ and higher electrical conductivity values. This may be used in combination with different patterning geometries to further assess the optical, electrical and mechanical properties of such conductive patterns. Moreover, the implementation of these inks in strain sensors and the characterization of the resulting materials and device performance is proposed as future research. Interesting perspectives from such investigations may include degradation studies of the devices, when subjected to humid and/or acidic operation conditions resembling wearable devices.

Regarding the fabrication of textured Al-doped $\mathrm{ZnO}$ patterned films using solution based inks and additive printing, the exploration of such a method using alternative metal-oxide materials systems is proposed as future work. Furthermore, the systematic investigation of the proposed wrinkling-texturing additional tuning pathway, through the administration of more or less energy during the sintering process should be conducted to characterize the heat-treatment routes, and establish the energy thresholds for attaining specific texturing degrees. Also, further investigation of the mechanical, optical and electrical properties of such films with different texturing and wrinkling degrees, may provide complementary information regarding the potential of CDW of sol-gel ink systems for microstructure engineering of metal-oxide systems. Specifically, the relationships between the films' wrinkle morphologies and their refractive index, mechanical flexibility and wear-resistant properties.

Higher resolution printing ( $5-100 \mu \mathrm{m})$ of the hybrid low viscosity ( $10-100 \mathrm{mPa} \cdot \mathrm{s}) \mathrm{TALH}: \mathrm{TiO}_{2}$ inks may be enabled by using co-solvent based formulations with different vapor pressure solvent combinations, as well as using hydrocolloids for their formulation. The expected outcomes from such approach are more control of the drying behavior of the printed microstructures (potentially reducing cracking), and the prevention of nozzle clogging due to sedimentation of the $\mathrm{TiO}_{2}$ primary particles. Z-potential measurements and $\mathrm{pH}$ tuning of the formulations is also expected to aid towards more kinetically stable inks.

Further experimental and simulation investigations on the hybrid $\mathrm{TiO}_{2}-\mathrm{TALH}$ foams' stability, characterizing the solvent drainage and bubble coalescence while printing and at rest after being extruded are proposed. Moreover, further studies on the use of xanthan gum, and similar hydrocolloids as gas stabilization agents of foams from hybrid titania suspensions is proposed. Specifically, the optimization of the amounts of such hydrocolloids for the gas stabilization and cellular shape retention even upon shearstress application, and the assessment of the stabilization effects when using different hydrocolloid blends, constitutes further work necessary for the transition from oil-based foam-ink formulations towards oil-free foams.

Additionally, the assessment of the mechanical properties of the "green" and sintered 3D printed $\mathrm{TiO}_{2}$ foams, and their dependence on the $\mathrm{TALH}: \mathrm{TiO}_{2}$ ratios, as well as their reinforcement with nanofibers 
and/or fillers such as carbon nanotubes and graphene flakes will provide further information about these systems towards their application as device components, and as UV-shielding coatings.

Simulation and experimental investigations of the light interactions with the mesostructured cellular titania scaffolds are proposed to provide further insights about the interesting relationships observed between the photocatalytic activity, surface area, macropore size and cellular configuration of the titania foams.

Additional research exploring the potential secondary-phase decorated/metal-oxide composites synthesis method, to demonstrate further control of the nucleated decorative materials shape, size and distribution; and plasmonic effects characterization, is also proposed as future work; including the demonstration of organic-molecule/metal-oxide composites from the proposed synthesis. Moreover, the use of this synthesis approach for the realization of alternative ceramic-metal mesoporous materials, such as those used in solid-oxide fuel cells is envisioned. Along this line, the exploration of high-temperature synthesis and operational regimes is required.

Lowering the resolution of the printed features, enabling more complex architectures, and investigating their curing/sintering, and release/accommodation of residual stress, under mild temperature conditions compatible with polymeric substrates is also an area of future work that may significantly contribute to the realization of novel applications.

Diverse applications demonstration, and device fabrication, characterization and optimization using the developed ink systems, is an interesting area of future research. Specifically, the use of optimized ink formulations and processing conditions for the realization of fully printable dye-sensitized solar cells, small molecule detection sensors, and bio-compatible scaffolds for growth of biological tissues is envisioned; taking advantage of the ample microstructuring potential of the investigated $\mathrm{TiO}_{2}$ material systems. Along this line, the study of interfacial phenomena between dissimilar materials, at the different stages (fabrication, operation, recycling-disposal) of such devices is proposed 


\section{Acknowledgements}

This work is the product of 3.5 years of constant reading, discussion and failure-success experimenting cycles. I would like to thank the advice and accompaniment of my academic adviser Dr. Konstantinos Sierros; who has constantly encouraged my development as a researcher and who has helped me see further horizons using his experience. I would also like to thank the advising committee members for their time, insight and discussion that has contributed to improving the quality and accurateness of my work. In this opportunity, I would like to recognize the importance of the NUE and IRES programs led by Dr. Sierros and Dr. Dimitris Korakakis, contributing to the development of my teaching and mentoring skills through the ENGR 393 class from 2015 to 2017. Additionally, I acknowledge the collaborative endeavours of Dr. Dimitris Korakakis, Dr. Edward Sabolsky, Dr. Terence Musho, Dr. Charter Stinespring, Dr. Cerasela Zoica Dinu, Dr. Rakesh Gupta and Dr. Sushant Agarwal, allowing me to use their labs and providing me with their valuable advice.

I would specially like to acknowledge the help and support of the FEST Lab members, especially from Derrick Bannerjee and Jacob Cordonier, who have welcome me from the first day, and have continuously helped facilitating my work. Special thanks to my undergraduate mentees for their help, understanding and patience, in particular to Domenic Cipollone, Lynnora Grant and Alana Valença de Andrade, I sincerely hope to have contributed to their success.

Many thanks to the WVU Mechanical and Aerospace Engineering department chairs Dr. Jacky Prucz and Dr. Victor Mucino for their valuable advice, and to the staff members for facilitating my work, especially Mrs. Katarzyna Sabolsky. Thanks to WVU Shared Research Facilities former and current managers Dr. Marcela Redigolo, Dr. Wei Ding, Dr. Qiang Wang, and Mr. Harley Hart.

Special thanks to my husband Diego Palacio for his constant support, and for the interesting discussion of my work, TEM results and characterization approaches. His accompaniment has helped me overcome many obstacles and face uncertainty, to reach this stage. Also, many thanks to my family for their love, support and listening, and particularly to my mother Rosario Arango, my sister Laura Torres Arango and my aunt Paulina Arango. 


\section{References}

1. Carter CB, Norton G. Ceramic Materials: Science and Engineering. SpringerLink : Bücher. 2013.722 p.

2. Green DJ, Colombo P. Cellular Ceramics: Intriguing Structures, Novel Properties, and Innovative Applications. Mater Res Soc. 2003;28:296-300.

3. Campbell CT, Grant AW, Starr DE, Parker SC, Bondzie VA. Model oxide-supported metal catalysts: Energetics, particle thicknesses, chemisorption and catalytic properties. Top Catal. 2000;14(1-4):43-51.

4. Hecht DS, Hu L, Irvin G. Emerging Transparent Electrodes Based on Thin Films of Carbon Nanotubes, Graphene, and Metallic Nanostructures. Adv Mater. 2011 Apr 5;23(13):1482-513.

5. Magdassi S, Grouchko M, Kamyshny A. Conductive Ink-Jet Inks for Plastic Electronics: Air Stable Copper Nanoparticles and Room Temperature Sintering. In: NIP25 and Digital Fabrication 2009. Society for Imaging Science and Technology; 2009. p. 611-3.

6. Magdassi S, Grouchko M, Berezin O, Kamyshny A. Triggering the sintering of silver nanoparticles at room temperature. ACS Nano. 2010;4(4):1943-8.

7. Zardetto V, Brown TM, Reale A, Di Carlo A. Substrates for flexible electronics: A practical investigation on the electrical, film flexibility, optical, temperature, and solvent resistance properties. J Polym Sci Part B Polym Phys. 2011;49(9):638-48.

8. Leterrier Y, Médico L, Demarco F, Månson J-AE, Betz U, Escolà MF, et al. Mechanical integrity of transparent conductive oxide films for flexible polymer-based displays. Thin Solid Films. 2004 Jul;460(1-2):156-66.

9. Sierros KA, Banerjee DA, Morris NJ, Cairns DR, Kortidis I, Kiriakidis G. Mechanical properties of ZnO thin films deposited on polyester substrates used in flexible device applications. Thin Solid Films. 2010 Oct;519(1):32530.

10. Muth JT, Dixon PG, Woish L, Gibson LJ, Lewis JA. Architected cellular ceramics with tailored stiffness via direct foam writing. Proc Natl Acad Sci. 2017 Feb 21;114(8):1832-7.

11. Studart R, Gonzenbach UT, Tervoort E, Gauckler LJ, Studart AR, Gonzenbach UT, et al. Processing routes to macroporous ceramics: A review. J Am Ceram Soc. 2006;89(6):1771-89.

12. Colombo P. Conventional and novel processing methods for cellular ceramics. Phil Trans R Soc A. 2006;(November 2005):109-24.

13. Kango S, Kalia S, Celli A, Njuguna J, Habibi Y, Kumar R. Surface modification of inorganic nanoparticles for development of organic-inorganic nanocomposites - A review. Prog Polym Sci. 2013;38(8):1232-61.

14. Sanchez-Dominguez M, Pemartin K, Boutonnet M. Preparation of inorganic nanoparticles in oil-in-water microemulsions: A soft and versatile approach. Curr Opin Colloid Interface Sci. 2012;17(5):297-305.

15. Formo E, Lee E, Campbell D, Xia Y. Functionalization of electrospun $\mathrm{TiO}_{2}$ nanofibers with Pt nanoparticles and nanowires for catalytic applications. Nano Lett. 2008;8(2):668-72.

16. Koo WT, Choi SJ, Kim SJ, Jang JS, Tuller HL, Kim ID. Heterogeneous Sensitization of Metal-Organic Framework Driven Metal@Metal Oxide Complex Catalysts on an Oxide Nanofiber Scaffold Toward Superior Gas Sensors. J Am Chem Soc. 2016;138(40):13431-7.

17. Knoezinger H. Specific poisoning and characterization studies of catalytically active oxide surfaces. 1976;25:184.

18. Linsebigler AL, Lu G, Yates JT. Photocatalysis on $\mathrm{TiO}_{2}$ Surfaces: Principles, Mechanisms, and Selected Results. Chem Rev. 1995 May 1;95(3):735-58. 
19. Jeong L, Park WH. Preparation and characterization of gelatin nanofibers containing silver nanoparticles. Int J Mol Sci. 2014 Apr 22;15(4):6857-79.

20. Chambers C, Stewart SB, Su B, Jenkinson HF, Sandy JR, Ireland AJ. Silver doped titanium dioxide nanoparticles as antimicrobial additives to dental polymers. Dent Mater. 2017;33(3):e115-23.

21. Betz U, Kharrazi Olsson M, Marthy J, Escolá MF, Atamny F. Thin films engineering of indium tin oxide: Large area flat panel displays application. Surf Coatings Technol. 2006 May;200(20-21):5751-9.

22. Stuecker JN, Cesarano J, Hirschfeld DA. Control of the viscous behavior of highly concentrated mullite suspensions for robocasting. J Mater Process Technol. 2003;142(2):318-25.

23. Smith GB, Granqvist CG. Green Nanotechnology. Boca Raton: Taylor and Francis; 2011.

24. Saion E, Gharibshahi E. Journal of Fundamental Sciences. 2011;7(1):6-11.

25. Gharibshahi E, Saion E. Influence of dose on particle size and optical properties of colloidal platinum nanoparticles. Int J Mol Sci. 2012;13(11):14723-41.

26. Gharibshahi L, Saion E, Gharibshahi E, Shaari A, Matori K. Structural and Optical Properties of Ag Nanoparticles Synthesized by Thermal Treatment Method. Materials (Basel). 2017;10(4):402.

27. Choi HC, Jung YM, Kim S Bin. Size effects in the Raman spectra of $\mathrm{TiO}_{2}$ nanoparticles. Vib Spectrosc. $2005 ; 37(1): 33-8$.

28. Hullavarad S, Hullavarad N, Look D, Claflin B. Persistent photoconductivity studies in nanostructured ZnO UV sensors. Nanoscale Res Lett. 2009;4(12):1421-7.

29. Hösel M, Dam HF, Krebs FC. Development of Lab-to-Fab Production Equipment Across Several Length Scales for Printed Energy Technologies, Including Solar Cells. Energy Technol. 2015;3(4):293-304.

30. Hench LL, West JK. The sol-gel process. Chem Rev. 1990;90(1):33-72.

31. Brinker C, Scherer G. Sol-Gel Science: The Physics and Chemistry of Sol-Gel Processing. 1990. p. 462.

32. Mann S, Burkett SL, Davis S a, Fowler CE, Mendelson NH, Sims SD, et al. Sol-Gel Synthesis of Organized Matter. Chem Mater. 1997;9(11):2300-10.

33. Danks AE, Hall SR, Schnepp Z. The evolution of "sol-gel" chemistry as a technique for materials synthesis. Mater Horizons. 2016;3:91-112.

34. Soler-Illia GJAA, Azzaroni O. Multifunctional hybrids by combining ordered mesoporous materials and macromolecular building blocks. Chem Soc Rev. 2011;40(2):1107-50.

35. Brinker JC, Lu Y, Sellinger A, Fan H. Evaporation-Induced Self-Assembly: Nanostructures Made Easy. Adv Mater. 1999;11(7):579-585.

36. Zampieri A, Colombo P, Mabande GTPGTP, Selvam T, Schwieger W, Scheffler F. Zeolite Coatings on Microcellular Ceramic Foams: A Novel Route to Microreactor and Microseparator Devices. Adv Mater. 2004;16(9-10):819-23.

37. Chen L, Huang C, Xu G, Hutton SL, Miao L. Macroporous $\mathrm{TiO}_{2}$ foam with mesoporous walls. Mater Charact. 2013 Jan;75:8-12.

38. Du J, Lai X, Yang N, Zhai J, Kisailus D, Su F, et al. Hierarchically Ordered Macro-Mesoporous TiO $\mathrm{Ti}_{2}-\mathrm{Graphene}$ Composite Films: Improved Mass Transfer, Reduced Charge Recombination, and Their Enhanced Photocatalytic Activities. ACS Nano. 2011 Jan 25;5(1):590-6.

39. Crossland EJW, Noel N, Sivaram V, Leijtens T, Alexander-Webber J a, Snaith HJ. Mesoporous TiO 2 single 
crystals delivering enhanced mobility and optoelectronic device performance. Nature. 2013;495(7440):2159.

40. Kambe S, Murakoshi K, Kitamura T, Wada Y, Yanagida S, Kominami H, et al. Mesoporous electrodes having tight agglomeration of single-phase anatase $\mathrm{TiO}_{2}$ nanocrystallites: application to dye-sensitized solar cells. Sol Energy Mater Sol Cells. 2000;61(4):427-41.

41. Chen X, Mao SS. Titanium Dioxide Nanomaterials: Synthesis, Properties, Modifications, and Applications. Chem Rev. 2007 Jul;107(7):2891-959.

42. Ye M, Zheng D, Lv M, Chen C, Lin C, Lin Z. Hierarchically structured nanotubes for highly efficient dyesensitized solar cells. Adv Mater. 2013;25(22):3039-44.

43. Ye M, Xin X, Lin C, Lin Z. High Efficiency Dye-Sensitized Solar Cells Based on Hierarchically Structured Nanotubes. Nano Lett. 2011 Aug 10;11(8):3214-20.

44. Titirici MM, Antonietti M, Thomas A. A generalized synthesis of metal oxide hollow spheres using a hydrothermal approach. Chem Mater. 2006;18(16):3808-12.

45. Murakami TN, Kijitori Y, Kawashima N, Miyasaka T. Low temperature preparation of mesoporous TiO films $^{2}$ for efficient dye-sensitized photoelectrode by chemical vapor deposition combined with UV light irradiation. J Photochem Photobiol A Chem. 2004 Jun;164(1-3):187-91.

46. Mahmood K, Swain BS, Amassian A. Highly efficient hybrid photovoltaics based on hyperbranched threedimensional $\mathrm{TiO}_{2}$ electron transporting materials. Adv Mater. 2015;27(18):2859-65.

47. Wen J, Wilkes GL. Organic/Inorganic Hybrid Network Materials by the Sol-Gel Approach. Chem Mater. 1996;8(8):1667-81.

48. Walsh BD, Mann S. Synthesis of Cellular Inorganic Films from Self-Organized Media. Adv Mater. $1997 ; 9(8): 658-62$.

49. Foo KL, Hashim U, Muhammad K, Voon CH. Sol-gel synthesized zinc oxide nanorods and their structural and optical investigation for optoelectronic application. Nanoscale Res Lett. 2014;9(1):429.

50. Lakshmi BB, Patrissi CJ, Martin CR. Sol-Gel Template Synthesis of Semiconductor Oxide Micro- and Nanostructures. Chem Mater. 1997;9(11):2544-50.

51. Pacholski C, Kornowski A, Weller H. Site-specific photodeposition of silver on ZnO nanorods. Angew Chemie - Int Ed. 2004;43(36):4774-7.

52. Liu Y, Wei S, Gao W. Ag/ZnO heterostructures and their photocatalytic activity under visible light: Effect of reducing medium. J Hazard Mater. 2015;287:59-68.

53. Lewera A, Timperman L, Roguska A, Alonso-Vante N. Metal-support interactions between nanosized Pt and metal oxides $\left(\mathrm{WO}_{3}\right.$ and $\left.\mathrm{TiO}_{2}\right)$ studied using X-ray photoelectron spectroscopy. J Phys Chem C. 2011;115(41):20153-9.

54. Chan SC, Barteau MA. Physico-chemical effects on the scale-up of Ag photodeposition on $\mathrm{TiO}_{2}$ nanoparticles. Top Catal. 2011;54(5-7):378-89.

55. Lu Q, Lu Z, Lu Y, Lv L, Ning Y, Yu H, et al. Photocatalytic synthesis and photovoltaic application of Ag-TiO 2 nanorod composites. Nano Lett. 2013;13(11):5698-702.

56. Qiao P, Zou S, Xu S, Liu J, Li Y, Ma G, et al. A general synthesis strategy of multi-metallic nanoparticles within mesoporous titania via in situ photo-deposition. J Mater Chem A. 2014;2(41):17321-8.

57. Baskaran S, Song L, Liu J, Chen YL, Graff GL. Titanium Oxide Thin Films on Organic Interfaces through Biomimetic Processing. J Am Ceram Soc. 1998;81(2):401-8. 
58. Mbonyiryivuze A, Zongo S, Diallo A, Bertrand S, Minani E. Titanium Dioxide Nanoparticles Biosynthesis for Dye Sensitized Solar Cells application : Review. Phys Mater Chem. 2015;3(1):12-7.

59. Hernández-Gordillo A, Hernández-Arana A, Campero A, Vera-Robles LI. Biomimetic Sol-Gel Synthesis of TiO 2 and $\mathrm{SiO}_{2}$ Nanostructures. Langmuir. 2014 Apr 15;30(14):4084-93.

60. Gutiérrez-Tauste D, Zumeta I, Vigil E, Hernández-Fenollosa MA, Domènech X, Ayllón JA. New lowtemperature preparation method of the $\mathrm{TiO}_{2}$ porous photoelectrode for dye-sensitized solar cells using UV irradiation. J Photochem Photobiol A Chem. 2005 Oct;175(2-3):165-71.

61. Di Giacomo F, Lucarelli G, Pescetelli S, Matteocci F, Razza S, Di Carlo A, et al. Device architectures with nanocrystalline mesoporous scaffolds and thin compact layers for flexible perovskite solar cells and modules. IEEE-NANO 2015 - 15th Int Conf Nanotechnol. 2016;739-42.

62. Senthilarasu S, Peiris TAN, García-Cañadas J, Wijayantha KGU. Preparation of Nanocrystalline TiO $\mathrm{Tl}_{2}$ Electrodes for Flexible Dye-Sensitized Solar Cells: Influence of Mechanical Compression. J Phys Chem C. 2012;116(36):19053-61.

63. Kim M-G. Low-temperature fabrication of high-performance metal oxide thin-film electronics via combustion processing. Nat Mater. 2011;10(5):382-8.

64. Hutchins KM, Rupasinghe TP, Ditzler LR, Swenson DC, Sander JRG, Baltrusaitis J, et al. Nanocrystals of a metalorganic complex exhibit remarkably high conductivity that increases in a single-crystal-to-single-crystal transformation. J Am Chem Soc. 2014;136(19):6778-81.

65. Oh Y, Lee S-N, Kim H-K, Kim J. UV-Assisted Chemical Sintering of Inkjet-Printed $\mathrm{TiO}_{2}$ Photoelectrodes for LowTemperature Flexible Dye-Sensitized Solar Cells. J Electrochem Soc. 2012 Aug 29;159(10):H777-81.

66. Kim H, Hwang $\mathrm{T}$. Effect of titanium isopropoxide addition in low-temperature cured $\mathrm{TiO}_{2}$ photoanode for a flexible DSSC. J Sol-Gel Sci Technol. 2014 Oct 17;72(1):67-73.

67. Allen ML, Aronniemi M, Mattila T, Alastalo A, Ojanperä K, Suhonen M, et al. Electrical sintering of nanoparticle structures. Nanotechnology. 2008;19(17):175201.

68. Alastalo AT, Mattila T, Allen ML, Aronniemi MJ, Leppäniemi JH, Ojanperä KA, et al. Rapid Electrical Sintering of Nanoparticle Stuctures. Mater Res Soc Symp Proc. 2009;1113.

69. Kumar A, Zhou C. The Race To Replace Tin-Doped Indium Oxide: Which Material Will Win? ACS Nano. 2010 Jan 26;4(1):11-4.

70. Kang M-G, Guo LJ. Nanoimprinted Semitransparent Metal Electrodes and Their Application in Organic LightEmitting Diodes. Adv Mater. 2007 May 21;19(10):1391-6.

71. Mariani P, Vesce L, Di Carlo A. The role of printing techniques for large-area dye sensitized solar cells. Semicond Sci Technol. 2015;30(10):104003.

72. Krebs FC. All solution roll-to-roll processed polymer solar cells free from indium-tin-oxide and vacuum coating steps. Org Electron. 2009 Aug;10(5):761-8.

73. Krebs FC. Fabrication and processing of polymer solar cells: A review of printing and coating techniques. Sol Energy Mater Sol Cells. 2009;93(4):394-412.

74. Tang H, Prasad K, Sanjinès R, Schmid PE, Lévy F. Electrical and optical properties of $\mathrm{TiO}_{2}$ anatase thin films. J Appl Phys. 1994 Feb 15;75(4):2042-7.

75. Torres Arango MA, Cipollone DT, Grant LO, Korakakis D, Sierros KA. Continuous-Flow Direct Writing of Hybrid $\mathrm{TiO}_{2}$ Flexible Photo-Electrodes: Processing, Microstructure and Functionality Interrelations. MRS Adv. 2017 Feb 27;2(18):1021-8. 
76. Sarah MSP, Musa MZ, Asiah MN, Rusop M. Electrical conductivity characteristics of $\mathrm{TiO}_{2}$ thin film. In: 2010 International Conference on Electronic Devices, Systems and Applications. IEEE; 2010. p. 361-4.

77. Mathews NRR, Morales ER, Cortés-Jacome MAA, Toledo Antonio JAA, Lévy F, Sierros KA, et al. $\mathrm{TiO}_{2}$ thin films - Influence of annealing temperature on structural, optical and photocatalytic properties. Sol Energy. 2009 Sep;83(9):1499-508.

78. Nowotny MK, Bak T, Nowotny J. Electrical properties and defect chemistry of $\mathrm{TiO}_{2}$ single crystal. I. Electrical conductivity. J Phys Chem B. 2006;110(33):16270-82.

79. Takikawa H, Matsui T, Sakakibara T, Bendavid A, Martin PJ. Properties of titanium oxide film prepared by reactive cathodic vacuum arc deposition. 1999;348:145-51.

80. Viseu TMR, Ferreira MIC. Morphological characterization of $\mathrm{TiO}_{2}$ thin films. 1999;52:4-9.

81. Lee HW, Lau SP, Wang YG, Tse KY, Hng HH, Tay BK. Structural, electrical and optical properties of Al-doped ZnO thin films prepared by filtered cathodic vacuum arc technique. J Cryst Growth. 2004;268(3-4 SPEC. ISS.):596-601.

82. Gahtar A, Rahal A, Benhaoua B, Benramache S. A comparative study on structural and optical properties of $\mathrm{ZnO}$ and $\mathrm{Al}$-doped $\mathrm{ZnO}$ thin films obtained by ultrasonic spray method using different solvents. Opt - Int J Light Electron Opt. 2014;125(14):3674-8.

83. Krebs FC, Jørgensen M, Norrman K, Hagemann O, Alstrup J, Nielsen TD, et al. A complete process for production of flexible large area polymer solar cells entirely using screen printing-First public demonstration. Sol Energy Mater Sol Cells. 2009;93(4):422-41.

84. Oh Y, Yoon HG, Lee S-N, Kim H-K, Kim J. Inkjet-printing of $\mathrm{TiO}_{2}$ co-solvent Ink: from uniform ink-droplet to $\mathrm{TiO}_{2}$ photoelectrode for dye-sensitized solar cells. J Electrochem Soc. 2011;159(1):B34-8.

85. Gibson I, Rosen D, Stucker B. Additive Manufacturing Technologies. Additive Manufacturing Technologies: 3D Printing, Rapid Prototyping, and Direct Digital Manufacturing, Second Edition. 2015. 1-498 p.

86. Song J, Edirisinghe M, Evans J. Formulation and multilayer jet printing of ceramic inks. J Am Ceram Soc. 1999;82(12):3374-80.

87. Sachs E, Cima M, Williams P, Brancazio D, Cornie J. Three dimensional printing: rapid tooling and prototypes directly from a CAD model. J Eng Ind. 1992;114(4):481.

88. Cherrington R, Hughes DJ, Senthilarasu S, Goodship V. Inkjet-printed $\mathrm{TiO}_{2}$ nanoparticles from aqueous solutions for dye-sensitized solar cells (DSSCs). Energy Technol. 2015 Aug;3(8):866-70.

89. Tekin E, Smith PJ, Schubert US. Inkjet printing as a deposition and patterning tool for polymers and inorganic particles. Soft Matter. 2008;4(4):703.

90. Huang Y, Leu MCC, Mazumder J, Donmez A. Additive manufacturing: current ctate, future potential, gaps and needs, and recommendations. J Manuf Sci Eng. 2015;137(1):14001.

91. Zocca A, Colombo P, Gomes CM, Günster J. Additive manufacturing of ceramics: issues, potentialities, and opportunities. Green DJ, editor. J Am Ceram Soc. 2015 Jul;98(7):1983-2001.

92. Colombo P, Schmidt J, Franchin G, Zocca A, Günster J. Additive manufacturing techniques for fabricating complex ceramic components from preceramic polymers. Am Ceram Soc Bull. 2017;96(3):16-23.

93. Raney JR, Lewis JA. Printing mesoscale architectures. MRS Bull. 2015 Nov 9;40(11):943-50.

94. Walker SB, Lewis JA. Reactive silver inks for patterning high-conductivity features at mild temperatures. J Am Chem Soc. 2012 Jan 25;134(3):1419-21. 
95. Cesarano J, Segalman R, Calvert P. Robocasting provides moldless fabrication from slurry deposition. Ceram Ind. 1998;148(4):94-101.

96. Smay JE, Cesarano J, Tuttle BA, Lewis JA. Piezoelectric properties of 3-X periodic $\mathrm{Pb}\left(\mathrm{Zr}_{\mathrm{x}} \mathrm{Ti}_{1-\mathrm{x}}\right) \mathrm{O}_{3}-\mathrm{polymer}$ composites. J Appl Phys. 2002;92(10):6119-27.

97. Paul BK, Panat R, Mastrangelo C, Kim D, Johnson D. Manufacturing of smart goods: current state, future potential and research recommendations. J Micro Nano-Manufacturing. 2016;1-12.

98. Zhakeyev A, Wang P, Zhang L, Shu W, Wang H, Xuan J. Additive manufacturing: unlocking the evolution of energy materials. Adv Sci. 2017;1700187:1700187.

99. Duoss EB, Weisgraber TH, Hearon K, Zhu C, Small IV W, Metz TR, et al. Three-dimensional printing of elastomeric, cellular architectures with negative stiffness. Adv Funct Mater. 2014;24(31):4905-13.

100. Pique A, Chrisey DB. Direct-write technologies for rapid prototyping applications: sensors, electronics and integrated power sources. San Diego: Academic Press; 2002.

101. Lewis JA. Direct ink writing of 3D functional materials. Adv Funct Mater. 2006 Nov 3;16(17):2193-204.

102. Hon KKB, Li L, Hutchings IM. Direct writing technology-advances and developments. CIRP Ann. 2008;57(2):601-20.

103. Lewis JA, Gratson GM. Direct writing in three dimensions. Mater Today. 2004 Jul;7(7-8):32-9.

104. Xu Q, Lv Y, Dong C, Sreeprased TS, Tian A, Zhang H, et al. Three-dimensional micro/nanoscale architectures: fabrication and applications. Nanoscale. 2015;7(25):10883-95.

105. Gratson GM, Lewis JA. Phase behavior and rheological properties of polyelectrolyte inks for direct-write assembly. Langmuir. 2005;21(1):457-64.

106. Xu M, Gratson GM, Duoss EB, Shepherd RF, Lewis JA. Biomimetic silicification of 3D polyamine-rich scaffolds assembled by direct ink writing. Soft Matter. 2006;2(3):205.

107. Eqtesadi S, Motealleh A, Miranda P, Lemos A, Rebelo A, Ferreira JMFFJMF. A simple recipe for direct writing complex 45S5 Bioglass ${ }^{\circledR}$ 3D scaffolds. Mater Lett. 2013 Feb;93:68-71.

108. Lewis JA. Colloidal processing of ceramics. 2000;59:2341-59.

109. Liu G, Zhou Y, Banga RS, Boya R, Brown KA, Chipre AJ, et al. The role of viscosity on polymer ink transport in dip-pen nanolithography. Chem Sci. 2013;4(5):2093.

110. Navratil J, Hamacek A, Reboun J, Soukup R. Perspective methods of creating conductive paths by Aerosol Jet Printing technology. In: 2015 38th International Spring Seminar on Electronics Technology (ISSE). IEEE; 2015. p. 36-9.

111. An BW, Kim K, Lee H, Kim SY, Shim Y, Lee DY, et al. High-resolution printing of 3D structures using an electrohydrodynamic inkjet with multiple functional inks. Adv Mater. 2015;27(29):4322-8.

112. Choi HW, Zhou T, Singh M, Jabbour GE. Recent developments and directions in printed nanomaterials. Nanoscale. 2015;7(8):3338-55.

113. Layani M, Gruchko M, Milo O, Balberg I, Azulay D, Magdassi S. Transparent conductive coatings by printing coffee ring arrays obtained at room temperature. ACS Nano. 2009;3(11):3537-42.

114. Liu Z, Su Y, Varahramyan K. Inkjet-printed silver conductors using silver nitrate ink and their electrical contacts with conducting polymers. Thin Solid Films. 2005 May;478(1-2):275-9.

115. Sun K, Wei T-S, Ahn BY, Seo JY, Dillon SJ, Lewis JA. 3D Printing of interdigitated Li-ion microbattery 
architectures. Adv Mater. 2013 Sep 6;25(33):4539-43.

116. Fu K, Wang Y, Yan C, Yao Y, Chen Y, Dai J, et al. Graphene oxide-based electrode inks for 3D-printed lithiumion batteries. Adv Mater. 2016;28(13):2587-94.

117. Kong YL, Tamargo IA, Kim H, Johnson BN, Gupta MK, Koh T-W, et al. 3D Printed quantum dot light-emitting diodes. Nano Lett. 2014 Dec 10;14(12):7017-23.

118. Mannoor MS, Jiang Z, James T, Kong YL, Malatesta KA, Soboyejo W, et al. A 3D Printed bionic ear. Nano Lett. 2013;13(6):2634-9.

119. Ho CC, Steingart D, Evans J, Wright P. Tailoring electrochemical capacitor energy storage using direct write dispenser printing. In: ECS Transactions. ECS; 2008. p. 35-47.

120. Zhu C, Liu T, Qian F, Han TYJ, Duoss EB, Kuntz JD, et al. Supercapacitors based on three-dimensional hierarchical graphene aerogels with periodic macropores. Nano Lett. 2016;16(6):3448-56.

121. Ahn BY, Duoss EB, Motala MJ, Guo X, Park S-I, Xiong Y, et al. Omnidirectional printing of flexible, stretchable, and spanning silver microelectrodes. Science. 2009 Mar 20;323(5921):1590-3.

122. Ahn BY, Lorang DJ, Lewis JA. Transparent conductive grids via direct writing of silver nanoparticle inks. Nanoscale. 2011;3(7):2700.

123. Torres Arango MA, Cokeley AM, Beard JJ, Sierros KA. Direct writing and electro-mechanical characterization of Ag micro-patterns on polymer substrates for flexible electronics. Thin Solid Films. 2015 Dec;596:167-73.

124. Duoss EB, Twardowski M, Lewis JA. Sol-gel inks for direct-write assembly of functional oxides. Adv Mater. 2007 Nov 5;19(21):3485-9.

125. Lewis JA, Duoss EB, Twardowski M. US007956102B2 Sol-Gel Inks. United States; 7956102B2, 2011.

126. Li Y, Li L, Li B. Direct ink writing of special-shaped structures based on $\mathrm{TiO}_{2}$ inks. Mod Phys Lett $\mathrm{B}$. 2016;30(11):1650212_1-1650212_7.

127. Li J-J, Li B, Peng Q-M, Zhou J, Li L-T. Fabrication and characterization of direct-written 3D $\mathrm{TiO}_{2}$ woodpile electromagnetic bandgap structures. Chinese Phys B. 2014;23(9):098104_1-098104_7.

128. Torres Arango MA, Valença de Andrade AS, Cipollone DT, Grant LO, Korakakis D, Sierros KA. Robotic deposition of $\mathrm{TiO}_{2}$ films on flexible substrates from hybrid inks: investigation of synthesis-processingmicrostructure-photocatalytic relationships. ACS Appl Mater Interfaces. 2016 Sep 21;8(37):24659-70.

129. Tohver V, Morissette SL, Lewis J a., Tuttle B a., Voigt J a., Dimos DB. Direct-write fabrication of zinc oxide varistors. J Am Ceram Soc. 2002;85(1):123-8.

130. Vunnam S, Ankireddy K, Kellar J, Cross W. Highly transparent and conductive Al-doped ZnO nanoparticulate thin films using direct write processing. Nanotechnology. 2014;25(19):195301.

131. Torres Arango MA, Abidakun OA, Korakakis D, Sierros KA. Tuning the crystalline microstructure of Al-doped ZnO using direct ink writing. Flex Print Electron. 2017 Aug 23;

132. Ahn BY, Lorang DJ, Duoss EB, Lewis JA. Direct-write assembly of microperiodic planar and spanning ITO microelectrodes. Chem Commun. 2010;46(38):7118-20.

133. Feilden E, Blanca EG-T, Giuliani F, Saiz E, Vandeperre L. Robocasting of structural ceramic parts with hydrogel inks. J Eur Ceram Soc. 2016 Aug;36(10):2525-33.

134. Rueschhoff L, Costakis W, Michie M, Youngblood J, Trice R. Additive manufacturing of dense ceramic parts via direct ink writing of aqueous alumina suspensions. Int J Appl Ceram Technol. 2016 Sep;13(5):821-30. 
135. Lis M, Plaut M, Zai A, Cipolle D, Russo J, Fedynyshyn T. High Performance, 3D-Printable dielectric nanocomposites for millimeter wave devices. ACS Appl Mater Interfaces. 2016;8(49):34019-26.

136. Lous GM, Cornejo I a., McNulty TF, Safari a., Danfortha SC. Fabrication of piezoelectric ceramic / polymer composite transducers using fused deposition of ceramics. MRS Proc. 1998;542:124-8.

137. Secor EB, Hersam MC. Emerging carbon and post-carbon nanomaterial inks for printed electronics. J Phys Chem Lett. 2015 Feb 19;6(4):620-6.

138. García-Tuñon E, Barg S, Franco J, Bell R, Eslava S, D’Elia E, et al. Printing in three dimensions with graphene. Adv Mater. 2015 Mar 11;27(10):1688-93.

139. Nesaei S, Rock M, Wang Y. Additive manufacturing with conductive, viscoelastic polymer composites: directink-writing of electrolytic and anodic poly (ethylene oxide) composites. J Manuf Sci Eng Addit. 2017;139(November):1-52.

140. Morissette SL, Lewis JA, Clem PG, Cesarano J, Dimos DB. Direct-write fabrication of $\mathrm{Pb}(\mathrm{Nb}, \mathrm{Zr}, \mathrm{Ti}) \mathrm{O}_{3}$ devices: influence of paste rheology on print morphology and component properties. J Am Ceram Soc. 2001 Nov;84(11):2462-8.

141. Tuttle BA, Smay JE, Cesarano J, Voigt JA, Scofield TW, Olson WR, et al. Robocast $\mathrm{Pb}\left(\mathrm{Zr}_{0.95} \mathrm{Ti}_{0.05}\right) \mathrm{O}_{3}$ ceramic monoliths and composites. J Am Ceram Soc. 2001 Apr;84(4):872-4.

142. Conrad JC, Ferreira SR, Yoshikawa J, Shepherd RF, Ahn BY, Lewis JA. Designing colloidal suspensions for directed materials assembly. Curr Opin Colloid Interface Sci. 2011;16(1):71-9.

143. Rao RB, Krafcik KL, Morales AM, Lewis JA. Microfabricated deposition nozzles for direct-write assembly of three-dimensional periodic structures. Adv Mater. 2005;17(3):289-93.

144. Dingeldein JC, Walczak KA, Swatowski BW, Friedrich CR, Middlebrook CT, Roggemann MC. Process characterization for direct dispense fabrication of polymer optical multi-mode waveguides. J Micromechanics Microengineering. 2013;23(7):75015.

145. Franks G V., Tallon C, Studart AR, Sesso ML, Leo S. Colloidal processing: enabling complex shaped ceramics with unique multiscale structures. J Am Ceram Soc. 2017 Feb;100(2):458-90.

146. Smay JE, Cesarano J, Lewis JA. Colloidal inks for directed assembly of 3-D periodic structures. Langmuir. 2002;18(14):5429-37.

147. Gratson GM, Xu M, Lewis JA. Direct writing of three-dimensional webs. Nature. 2004 Mar 25;428:386.

148. Herschel WH, Bulkley R. Konsistenzmessungen von Gummi-Benzollosungen. Kolloid-Z. 1926 Aug;39(4):291300.

149. Malkin AY, Isayev Al. Rheology - Concepts, Methods, and Applications. Second. Toronto: ChemTec Publishing; 2012. 143-146 p.

150. Gupta RK. Introduction to Polymer Rheology. In: Gupta RK, editor. Polymer and Composite Rheology. Second. New York: Marcel Dekker; 2000. p. 1-12.

151. Gupta RK. Yield Stress, Wall Slip, Particle Migration, and Other Observations with Multiphase Sstems. In: Gupta RK, editor. Polymer and Composite Rheology. Second. New York: Marcel Dekker; 2000. p. 197-223.

152. Sommer MR, Schaffner M, Carnelli D, Studart AR. 3D Printing of hierarchical silk fibroin structures. ACS Appl Mater Interfaces. 2016;8(50):34677-85.

153. Maiti A, Small W, Lewicki JP, Weisgraber TH, Duoss EB, Chinn SC, et al. 3D printed cellular solid outperforms traditional stochastic foam in long-term mechanical response. Sci Rep. 2016 Jul 27;6(1):24871. 
154. Minas C, Carnelli D, Tervoort E, Studart AR. 3D Printing of emulsions and foams into hierarchical porous ceramics. Adv Mater. 2016;28(45):9993-9.

155. Sommer MR, Alison L, Minas C, Tervoort E, Rühs PA, Studart AR. 3D printing of concentrated emulsions into multiphase biocompatible soft materials. Soft Matter. 2017;13(9):1794-803.

156. Everitt P, Taylor J. Reticulated foams expand the boundaries of cellular solids. MRS Bull. 2017;42:266-7.

157. Chen C, Long M, Cai W, Zhou B. Synthesis and photocatalytic application of hierarchical macroporous $\mathrm{TiO}_{2}$ with mesocellular foam structure using eggshell membrane as template. 2010 4th Int Conf Bioinforma Biomed Eng iCBBE 2010. 2010;2-5.

158. Ibhadon AO, Greenway GM, Yue $\mathrm{Y}$, Falaras $\mathrm{P}$, Tsoukleris $\mathrm{D}$. The photocatalytic activity of $\mathrm{TiO}_{2}$ foam and surface modified binary oxide titania nanoparticles. J Photochem Photobiol A Chem. 2008;197(2-3):321-8.

159. Tytgat $\mathrm{T}$, Smits $\mathrm{M}$, Lenaerts $\mathrm{S}$, Verbruggen SW. Immobilization of $\mathrm{TiO}_{2}$ into self-supporting photocatalytic foam: Influence of calcination temperature. Int J Appl Ceram Technol. 2014;11(4):714-22.

160. Chen L, Huang C, Xu G, Hutton SL, Miao L. Macroporous $\mathrm{TiO}_{2}$ foam with mesoporous walls. Mater Charact. 2013;75:8-12.

161. Jiang $\mathrm{Y}$, Chen $\mathrm{G}, \mathrm{Xu} \mathrm{X}$, Chen X, Deng S, Smirnov S, et al. Direct growth of mesoporous anatase $\mathrm{TiO}_{2}$ on nickel foam by soft template method as binder-free anode for lithium-ion batteries. RSC Adv. 2014;4(90):4893842.

162. Krishnappa M, Souza VS, Ganganagappa N, Scholten JD, Teixeira SR, Dupont J, et al. Mesoporous foam $\mathrm{TiO}_{2}$ nanomaterials for effective hydrogen production. Chem - A Eur J. 2015 Dec 1;21(49):17624-30.

163. Zhou $M$, Lin $T$, Huang $F$, Zhong $Y$, Wang $Z$, Tang $Y$, et al. Highly conductive porous graphene/ceramic composites for heat transfer and thermal energy storage. Adv Funct Mater. 2013;23(18):2263-9.

164. Rezwan K, Chen QZ, Blaker JJ, Boccaccini AR. Biodegradable and bioactive porous polymer/inorganic composite scaffolds for bone tissue engineering. Biomaterials. 2006;27(18):3413-31.

165. Haugen $\mathrm{H}$, Will J, Köhler A, Hopfner U, Aigner J, Wintermantel E. Ceramic $\mathrm{TiO}_{2}$-foams: characterisation of a potential scaffold. J Eur Ceram Soc. 2004;24(4):661-8.

166. Narkevica I, Stradina L, Yakushin V, Ozolins J. Preparation and characterization of porous titania ceramic scaffolds. Mater Sci Appl Chem. 2015;31:5.

167. Pokhrel A, Seo DN, Lee ST, Kim IJ. Processing of porous ceramics by direct foaming: A review. J Korean Ceram Soc. 2013;50(2):93-102.

168. Gonzenbach UT, Studart AR, Tervoort E, Gauckler L. Ultrastable particle-stabilized foams. Angew Chemie Int Ed. 2006 May 19;45(21):3526-30.

169. Imhof A, Pine DJ. Preparation of titania foams. Adv Mater. 1999;11(4):311-4.

170. Collins AM, Spickermann C, Mann S. Synthesis of titania hollow microspheres using non-aqueous emulsions. J Mater Chem. 2003;13(5):1112-4.

171. Carn F, Colin A, Achard MF, Deleuze H, Sanchez C, Backov R. Anatase and rutile $\mathrm{TiO}_{2}$ macrocellular foams: air-liquid foaming sol-gel process towards controlling cell sizes, morphologies, and topologies. Adv Mater. 2005;17(1):62-6.

172. Watson WJW. How do the fine chemical, pharmaceutical, and related industries approach green chemistry and sustainability? Green Chem. 2012;14(2):251-9.

173. Huang SH, Liu P, Mokasdar A, Hou L. Additive manufacturing and its societal impact: a literature review. Int 
J Adv Manuf Technol. 2013;67(5-8):1191-203.

174. Anastas PT, Warner JC. Green Chemistry : theory and practice. 1998.

175. Anastas PT, Zimmerman JB. Peer Reviewed: Design Through the 12 Principles of Green Engineering. Environ Sci Technol. 2003 Mar;37(5):94A-101A.

176. Allen DT, Hwang B-J, Licence P, Pradeep T, Subramaniam B. Advancing the use of sustainability metrics. ACS Sustain Chem Eng. 2015 Oct 5;3(10):2359-60.

177. Mani M, Lyons KW, Gupta SK. Sustainability characterization for additive manufacturing. J Res Natl Inst Stand Technol. 2014;119:419-28.

178. O'Brien S, Çopuroglu M, Tassie P, Nolan MG, Hamilton JA, Povey I, et al. The effect of dopants on the morphology, microstructure and electrical properties of transparent zinc oxide films prepared by the sol-gel method. Thin Solid Films. 2011;520(4):1174-7.

179. Banerjee DA, Kessman AJ, Cairns DR, Sierros KA. Tribology of silica nanoparticle-reinforced, hydrophobic solgel composite coatings. Surf Coatings Technol. 2014;260:214-9.

180. Monoethanolamine, Diethanolamine, Triethanolamine. The Dow Chemical Company. Midland, Michigan; 2003. p. 1-21.

181. Lin VS. A Porous Silicon-Based Optical Interferometric Biosensor. Science. 1997 Oct 31;278(5339):840-3.

182. Liu X, Zhou L, Liu F, Ji M, Tang W, Pang M, et al. Exponential growth of layer-by-layer assembled coatings with well-dispersed ultrafine nanofillers: a facile route to scratch-resistant and transparent hybrid coatings. J Mater Chem. 2010;20(36):7721.

183. Langford JI, Wilson AJC. Scherrer after sixty years: A survey and some new results in the determination of crystallite size. J Appl Crystallogr. 1978 Apr 1;11(2):102-13.

184. Adam R, Eveson RW, MacDonald WA. COATED AND PLANARISED POLYMERIC FILMS. US; US 20100322867A1, 2011.

185. Fortunato E, Nunes P, Marques A, Costa D, Águas H, Ferreira I, et al. Transparent, conductive ZnO:Al thin film deposited on polymer substrates by RF magnetron sputtering. Surf Coatings Technol. 2002 Mar;151152:247-51.

186. Cairns DR, Witte RP, Sparacin DK, Sachsman SM, Paine DC, Crawford GP, et al. Strain-dependent electrical resistance of tin-doped indium oxide on polymer substrates. Appl Phys Lett. 2000;76(11):1425.

187. Kim B-J, Haas T, Friederich A, Lee J-H, Nam D-H, Binder JR, et al. Improving mechanical fatigue resistance by optimizing the nanoporous structure of inkjet-printed Ag electrodes for flexible devices. Nanotechnology. 2014;25(12):125706.

188. Smith DR, Fickett FR. Low-temperature properties of silver. 1995;100(2).

189. Greer JR, Nix WD. Nanoscale gold pillars strengthened through dislocation starvation. Phys Rev B - Condens Matter Mater Phys. 2006;73(24):1-6.

190. Abidakun OA. Direct Writing of Aluminum Doped Zinc Oxide for Optoelectronic and Energy Device Applications. West Virginia University; 2015.

191. Liang T-X, Sun WZ, Wang L-D, Wang YH, Li H-D. Effect of surface energies on screen printing resolution. IEEE Trans Components, Packag Manuf Technol Part B. 1996 May;19(2):423-6.

192. Fernández L, Sánchez M, Carmona FJ, Palacio L, Calvo JI, Hernández A, et al. Analysis of the grafting process of PVP on a silicon surface by AFM and contact angle. Langmuir. 2011 Sep 20;27(18):11636-49. 
193. van Oss CJ. Interfacial Forces in Aqueous Media. In Taylor \& Francis Group, LLC; 2006.

194. Yaws CL. Yaws' Thermophysical Properties of Chemicals and Hydrocarbons (Electronic Edition). Norwich, N.Y: Knovel; 2010.

195. Bolten D, Türk M. Experimental study on the surface tension, density, and viscosity of aqueous poly(vinylpyrrolidone) solutions. J Chem Eng Data. 2011 Mar 10;56(3):582-8.

196. Lotgering FK. Topotactical reactions with ferrimagnetic oxides having hexagonal crystal structures - I. J Inorg Nucl Chem. 1959 Feb;9(2):113-23.

197. Sardela M, Mauro S. Practical Materials Characterization. Sardela M, editor. New York, NY: Springer New York; 2014.

198. Lu JG, Fujita S, Kawaharamura T, Nishinaka H, Kamada Y, Ohshima T, et al. Carrier concentration dependence of band gap shift in n-type ZnO:Al films. J Appl Phys. 2007 Apr 15;101(8):83705.

199. Hanada T. Basic Properties of ZnO, GaN, and Related Materials. In: Yao T, Hong S-K, editors. Oxide and Nitride Semiconductors. Berlin, Heidelberg: Springer Berlin Heidelberg; 2009. p. 1-19. (Advances in Materials Research).

200. Chen KJ, Fang TH, Hung FY, Ji LW, Chang SJ, Young SJ, et al. The crystallization and physical properties of Aldoped ZnO nanoparticles. Appl Surf Sci. 2008;254(18):5791-5.

201. Hu A, Griesing S, Rybachuk M, Lu Q-B, Duley WW. Nanobuckling and x-ray photoelectron spectra of carbynerich tetrahedral carbon films deposited by femtosecond laser ablation at cryogenic temperatures. J Appl Phys. 2007 Oct;102(7):74311.

202. Bauermann LP, Campo A Del, Bill J, Aldinger F. Heterogeneous nucleation of ZnO using gelatin as the organic matrix. Chem Mater. 2006;18(8):2016-20.

203. Grinis L, Kotlyar S, Rühie S, Grinblat J, Zaban A. Conformal nano-Sized inorganic coatings on mesoporous $\mathrm{TiO}_{2}$ films for low-Temperature dye-Sensitized solar cell fabrication. Adv Funct Mater. 2010;20(2):282-8.

204. Kim S-J, Lee K, Kim JH, Lee N-H, Kim S-J. Preparation of brookite phase $\mathrm{TiO}_{2}$ colloidal sol for thin film coating. Mater Lett. 2006 Feb;60(3):364-7.

205. Möckel H, Giersig M, Willig F. Formation of uniform size anatase nanocrystals from bis(ammonium lactato)titanium dihydroxide by thermohydrolysis. J Mater Chem. 1999;9(12):3051-6.

206. Heo SY, Park JT, Patel R, Kim JK, Kim JH. One-step Fabrication of Crack-free, Hierarchically-ordered TiO ${ }_{2}$ films via self-assembly of polystyrene bead and preformed $\mathrm{TiO}_{2}$. Electrochim Acta. 2014;117:521-7.

207. Hu H, Larson RG. Marangoni effect reverses coffee-ring depositions. J Phys Chem B. 2006 Apr;110(14):70904.

208. Gribb AA, Banfield JF. Particle size effects on transformation kinetics and phase stability in nanocrystalline $\mathrm{TiO}_{2}$. Am Mineral. 1997;82(7-8):717-28.

209. Ohtani B, Prieto-Mahaney OO, Li D, Abe R. What is Degussa (Evonik) P25? Crystalline composition analysis, reconstruction from isolated pure particles and photocatalytic activity test. J Photochem Photobiol A Chem. 2010 Dec;216(2-3):179-82.

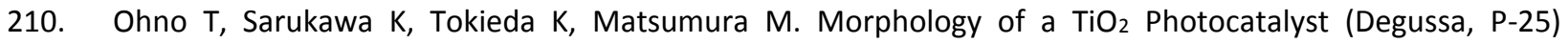
Consisting of Anatase and Rutile Crystalline Phases. J Catal. 2001;203(1):82-6.

211. Zulkifili ANB, Kento T, Daiki M, Fujiki A. The basic research on the dye-sensitized solar cells (DSSC). J Clean Energy Technol. 2015;3(5):382-7. 
212. Siqueira IR, Rebouças RB, Carvalho MS. Particle migration and alignment in slot coating flows of elongated particle suspensions. AIChE J. 2017 Jul;63(7):3187-98.

213. Zhang F, Chen J, Zhang X, Gao W, Jin R, Guan N, et al. Synthesis of titania-supported platinum catalyst: the effect of $\mathrm{pH}$ on morphology control and valence state during photodeposition. Langmuir. 2004;20(21):932934.

214. Ohsaka T, Izumi F, Fujiki Y. Raman spectrum of anatase, TiO 2 . J Raman Spectrosc. 1978 Dec;7(6):321-4.

215. Balachandran U, Eror NG. Raman spectra of titanium dioxide. J Solid State Chem. 1982 May;42(3):276-82.

216. Hardcastle F. Raman spectroscopy of titania $\left(\mathrm{TiO}_{2}\right)$ nanotubular water-splitting catalysts. J Ark Acad Sci. 2011;65:43-8.

217. Mcauley S. Investigation of the Formation and Photo-activity of Titanium Dioxide and Polyelectrolytes. University of Toronto; 2010.

218. Zhang J, Li M, Feng Z, Chen J, Li C. UV Raman spectroscopic study on TiOs2 . I. Phase transformation at the surface and in the bulk. J Phys Chem B. 2006 Jan;110(2):927-35.

219. Xu CY, Zhang PX, Yan L. Blue shift of Raman peak from coated $\mathrm{TiO}_{2}$ nanoparticles. J Raman Spectrosc. 2001 Oct 1;32(10):862-5.

220. Nkosi SS, Kortidis I, Motaung DE, Makgwane PR, Ndwandwe OM, Ray SS, et al. An instant photo-excited electrons relaxation on the photo-degradation properties of $\mathrm{TiO}_{2-x}$ films. J Photochem Photobiol A Chem. 2014;293:72-80.

221. Godlewski S, Szymonski M. Adsorption and self-assembly of large polycyclic molecules on the surfaces of $\mathrm{TiO}_{2}$ single crystals. Int J Mol Sci. 2013;14(2):2946-66.

222. Nuesch F, Rothberg LJ, Forsythe EW, Toan Le Q, Gao Y. A photoelectron spectroscopy study on the indium tin oxide treatment by acids and bases. Appl Phys Lett. 1999;74(1999):880-2.

223. Schwartz J, Bruner EL, Koch N, Span AR, Bernasek SL, Kahn A. Controlling the work function of indium tin oxide: differentiating dipolar from local surface effects. Synth Met. 2003;138(1-2):223-7.

224. Brumbach M, Veneman PA, Matrikar FS, Schulmeyer T, Simmonds A, Xia W, et al. Surface composition and electrical and electrochemical properties of freshly deposited and acid-etched indium tin oxide electrodes. Langmuir. 2007;23(22):11089-99.

225. Yang W, Li S, Gutowski V. Multi-functional nano-materials for advanced automotive applications. In: PMA 2007 \& 19th SRC 2007. Bratislava; 2007. p. s3-5.

226. Tang H, Prasad K, Sanjines R, Schmid PE, Levy F. Electrical and optical properties of $\mathrm{TiO}_{2}$ anatase thin films. J Appl Phys. 1994;75(4):2042-7.

227. Logothetidis S. Handbook of flexible organic electronics : materials, manufacturing and applications.

228. Pickering SU. Emulsions. J Chem Soc, Trans. 1907;91:2001-21.

229. Binks BP, Lumsdon SO. Influence of particle wettability on the type and stability of surfactant-free emulsions. Langmuir. 2000;16(23):8622-31.

230. Aveyard R, Binks BP, Clint JH. Emulsions stabilised solely by colloidal particles. Adv Colloid Interface Sci. 2003;100-102:503-46.

231. Agarwal S, Phuoc TX, Soong Y, Martello D, Gupta RK. Nanoparticle-stabilised invert emulsion drilling fluids for deep-hole drilling of oil and gas. Can J Chem Eng. 2013;91(10):1641-9. 
232. Kalustian P. Pharmaceutical and cosmetic uses of palm and lauric products. J Am Oil Chem Soc. 1985;62(2):431-3.

233. Magg H. Fatty acid derivatives: important surfactants for household, cosmetic and industrial purposes. J Am Oil Chem Soc. 1984;61(2):259-67.

234. Wang $\mathrm{P}$, Wang J, Wang $\mathrm{X}, \mathrm{Yu} \mathrm{H}, \mathrm{Yu}$ J, Lei $\mathrm{M}$, et al. One-step synthesis of easy-recycling $\mathrm{TiO}_{2}-\mathrm{rGO}$ nanocomposite photocatalysts with enhanced photocatalytic activity. Appl Catal B Environ. 2013 Mar;132133:452-9.

235. Chong MN, Jin B, Chow CWK, Saint C. Recent developments in photocatalytic water treatment technology: A review. Water Res. 2010;44(10):2997-3027.

236. Saint-Jalmes A. Physical chemistry in foam drainage and coarsening. Soft Matter. 2006;2(10):836-49.

237. Gupta RK. Gas-Containing Melts and Foams. In: Gupta RK, editor. Polymer and Composite Rheology. Second. New York: Marcel Dekker; 2000. p. 304-15.

238. Buscall R, McGowan JI, Morton-Jones AJ. The rheology of concentrated dispersions of weakly attracting colloidal particles with and without wall slip. J Rheol (N Y N Y). 1993 Jul;37(4):621-41.

239. Gandolfo FG, Rosano HL. Interbubble gas diffusion and the stability of foams. J Colloid Interface Sci. $1997 ; 194(1): 31-6$.

240. Gonzenbach UT, Studart AR, Tervoort E, Gauckler LJ. Macroporous ceramics from particle-stabilized wet foams. J Am Ceram Soc. 2007 Jan;90(1):16-22.

241. Chuanuwatanakul C, Tallon C, Dunstana DE, Franks G V. Controlling the microstructure of ceramic particle stabilized foams: influence of contact angle and particle aggregation. Soft Matter. 2011;7:11464-74.

242. Nakahara A, Matsuo Y. Transition in the pattern of cracks resulting from memory effects in paste. Phys Rev E. 2006;74:045102(1-4).

243. Compton BG, Lewis JA. 3D-printing of lightweight cellular composites. Adv Mater. 2014;26(34):5930-5.

244. Trost BM. The Atom Economy - A Search for Synthetic Efficiency. Science (80- ). 1991;254(5037):1471-7.

245. Tabone MD, Cregg J, Beckman E, Landis AE. Sustainability metrics: life cycle assessment and green design in polymers. Environ Sci Technol. 2010;44(21):8264-9.

246. Santanach Carreras E, Chabert F, Dunstan DE, Franks GV. Avoiding “mud" cracks during drying of thin films from aqueous colloidal suspensions. J Colloid Interface Sci. 2007 Sep;313(1):160-8.

247. Di Valentin C, Finazzi E, Pacchioni G, Selloni A, Livraghi S, Paganini MC, et al. N-doped TiO 2 : theory and experiment. Chem Phys. 2007;339(1-3):44-56.

248. Dickinson E. Hydrocolloids at interfaces and the influence on the properties of dispersed systems. Food Hydrocoll. 2003;17(1):25-39.

249. Dickinson E. Biopolymer-based particles as stabilizing agents for emulsions and foams. Food Hydrocoll. 2017;68:219-31.

250. Glicksman M. Food Hydrocolloids. Glicksman M, editor. Boca Raton: CRC Press; 1982.

251. Katzbauer B. Properties and applications of xanthan gum. Polym Degrad Stab. 1998;59(1-3):81-4.

252. Wu MC, Hiltunen J, Sápi A, Avila A, Larsson W, Liao HC, et al. Nitrogen-doped anatase nanofibers decorated with noble metal nanoparticles for photocatalytic production of hydrogen. ACS Nano. 2011;5(6):5025-30.

253. Wu MC, Sápi A, Avila A, Szabó M, Hiltunen J, Huuhtanen M, et al. Enhanced photocatalytic activity of TiO 2 
nanofibers and their flexible composite films: decomposition of organic dyes and efficient $\mathrm{H}_{2}$ generation from ethanol-water mixtures. Nano Res. 2011;4(4):360-9.

254. Pu YC, Wang G, Chang K Der, Ling Y, Lin YK, Fitzmorris BC, et al. Au nanostructure-decorated $\mathrm{TiO}_{2}$ nanowires exhibiting photoactivity across entire UV-visible region for photoelectrochemical water splitting. Nano Lett. 2013;13(8):3817-23.

255. Xiao FX, Hung SF, Miao J, Wang HY, Yang H, Liu B. Metal-cluster-decorated $\mathrm{TiO}_{2}$ nanotube arrays: A composite heterostructure toward versatile photocatalytic and photoelectrochemical applications. Small. 2015;11(5):554-67.

256. Bamwenda GR, Tsubota S, Nakamura T, Haruta M. Photoassisted hydrogen production from a water-ethanol solution: a comparison of activities of $\mathrm{Au} / \mathrm{TiO}_{2}$ and $\mathrm{Pt} / \mathrm{TiO}_{2}$. J Photochem Photobiol A Chem. 1995;89(2):17789.

257. Yu J, Qi L, Jaroniec M. Hydrogen production by photocatalytic water splitting over $\mathrm{Pt} / \mathrm{TiO}_{2}$ nanosheets with exposed (001) facets. J Phys Chem C. 2010;114(1):13118-25.

258. Sclafani A, Mozzanega M-N, Pichat P. Effect of silver deposits on the photocatalytic activity of titanium dioxide samples for the dehydrogenation or oxidation of 2-propanol. J Photochem Photobiol A Chem. 1991 Jul;59(2):181-9.

259. Pearson A, Zheng H, Kalantar-Zadeh K, Bhargava SK, Bansal V. Decoration of $\mathrm{TiO}_{2}$ nanotubes with metal nanoparticles using polyoxometalate as a UV-switchable reducing agent for enhanced visible and solar light photocatalysis. Langmuir. 2012;28(40):14470-5.

260. Zhang Q, Lima DQ, Lee I, Zaera F, Chi M, Yin Y. A highly active titanium dioxide based visible-light photocatalyst with nonmetal doping and plasmonic metal decoration. Angew Chemie - Int Ed. 2011;50(31):7088-92.

261. Es-Souni M, Es-Souni M, Habouti S, Pfeiffer N, Lahmar A, Dietze M, et al. Brookite formation in $\mathrm{TiO}_{2}-\mathrm{Ag}$ nanocomposites and visible-light-induced templated growth of $\mathrm{Ag}$ nanostructures in $\mathrm{TiO}_{2}$. Adv Funct Mater. 2010;20(3):377-85.

262. Chan SC, Barteau MA. Preparation of highly uniform $\mathrm{Ag} / \mathrm{TiO}_{2}$ and $\mathrm{Au} / \mathrm{TiO}_{2}$ supported nanoparticle catalysts by photodeposition. Langmuir. 2005;21(12):5588-95.

263. Lakshminarasimhan N, Bokare AD, Choi W. Effect of agglomerated state in mesoporous $\mathrm{TiO}_{2}$ on the morphology of photodeposited Pt and photocatalytic activity. J Phys Chem C. 2012;116(33):17531-9.

264. Zhou N, López-Puente V, Wang Q, Polavarapu L, Pastoriza-Santos I, Xu Q-H. Plasmon-enhanced light harvesting: applications in enhanced photocatalysis, photodynamic therapy and photovoltaics. RSC Adv. 2015;5(37):29076-97.

265. Zhang Z, Wang Z, Cao SW, Xue C. Au/Pt nanoparticle-decorated $\mathrm{TiO}_{2}$ nanofibers with plasmon-enhanced photocatalytic activities for solar-to-fuel conversion. J Phys Chem C. 2013;117(49):25939-47.

266. Clavero C. Plasmon-induced hot-electron generation at nanoparticle/metal-oxide interfaces for photovoltaic and photocatalytic devices. Nat Photonics. 2014;8(2):95-103.

267. Lan MY, Liu CP, Huang HH, Lee SW. Both enhanced biocompatibility and antibacterial activity in Ag-decorated $\mathrm{TiO}_{2}$ nanotubes. PLoS One. 2013;8(10):4-11.

268. Yu B, Leung KM, Guo $Q$, Lau WM, Yang J. Synthesis of $\mathrm{Ag}-\mathrm{TiO}_{2}$ composite nano thin film for antimicrobial application. Nanotechnology. 2011;22(11):115603.

269. Piwoński I, Kdzioła K, Kisielewska A, Soliwoda K, Wolszczak M, Lisowska K, et al. The effect of the deposition parameters on size, distribution and antimicrobial properties of photoinduced silver nanoparticles on titania 
coatings. Appl Surf Sci. 2011;257(16):7076-82.

270. O'Regan B, Grätzel M. A low-cost, high-efficiency solar cell based on dye-sensitized colloidal $\mathrm{TiO}_{2}$ films. Nature. 1991 Oct 24;353(6346):737-40.

271. Ingham CJ, ter Maat J, de Vos WM. Where bio meets nano: the many uses for nanoporous aluminum oxide in biotechnology. Biotechnol Adv. 2012;30(5):1089-99.

272. Saha D, Bhattacharya S. Hydrocolloids as thickening and gelling agents in food: a critical review. J Food Sci Technol. 2010;47(6):587-97.

273. Song KW, Kim YS, Chang GS. Rheology of concentrated xanthan gum solutions: steady shear flow behavior. Fibers Polym. 2006;7(2):129-38.

274. Pettitt DJ. Xanthan Gum. In: Glicksman M, editor. Food Hydrocolloids Volume I. Boca Raton: CRC Press; 1982. p. 127-49.

275. García-Ochoa F, Santos VE, Casas JA, Gómez E. Xanthan gum: production, recovery, and properties. Biotechnol Adv. 2000;18(7):549-79.

276. Torres Arango MA, Kwakye-Ackah D, Agarwal S, Gupta RK, Sierros KA. Environmentally friendly engineering and three-dimensional printing of $\mathrm{TiO}_{2}$ hierarchical mesoporous cellular architectures. ACS Sustain Chem Eng. 2017 Oct 3;acssuschemeng.7b02450.

277. Ohko Y, Tatsuma T, Fujii T, Naoi K, Niwa C, Kubota Y, et al. Multicolour photochromism of $\mathrm{TiO}_{2}$ films loaded with silver nanoparticles. Nat Mater. 2003;2(1):29-31.

278. Tauc J. Optical properties and electronic structure of amorphous Ge and Si. MRS Bull. 1968;3:37-46.

279. Wenderich K, Mul G. Methods, mechanism, and applications of photodeposition in photocatalysis: a review. Chem Rev. 2016;116(23):14587-619.

280. Prakash J, Kumar P, Harris RA, Swart C. Synthesis, characterization and multifunctional properties of plasmonic $\mathrm{Ag}-\mathrm{TiO}_{2}$ nanocomposites. Nanotechnology. 27(35):1-20.

281. Xin B, Jing L, Ren Z, Wang B, Fu H. Effects of simultaneously doped and deposited Ag on the photocatalytic activity and surface states of $\mathrm{TiO}_{2}$. J Phys Chem B. 2005;109(7):2805-9.

282. Matsumoto $\mathrm{Y}$, Ida $\mathrm{S}$, Inoue T. Photodeposition of metal and metal oxide at the $\mathrm{TiO}_{\mathrm{x}}$ nanosheet to observe the photocatalytic active site. J Phys Chem C. 2008;112(31):11614-6.

283. Wagner CD. Auger lines in x-ray photoelectron spectrometry. Anal Chem. 1972;44(6):967-73.

284. Gaarenstroom SW, Winograd N. Initial and final state effects in the ESCA spectra of cadmium and silver oxides. J Chem Phys. 1977;67(8):3500-6.

285. Seah MP. AES: energy calibration of electron spectrometers. IV. A re-evaluation of the reference energies. J Electron Spectros Relat Phenomena. 1998;97(April 1988):235-41.

286. Schön G, Tummavuori J, Lindström B, Enzell CR, Enzell CR, Swahn C-G. ESCA Studies of Ag, $\mathrm{Ag}_{2} \mathrm{O}$ and AgO. Vol. 27, Acta Chemica Scandinavica. 1973. p. 2623-33.

287. Ferraria AM, Carapeto AP, Maria A. X-ray photoelectron spectroscopy: silver salts revisited. 2012;86:198891.

288. de Farias RF. Synthesis of $\mathrm{TiO}_{2}$ (anatase) by sol-gel process performed in metal chlorides saturated aqueous solutions. J Colloid Interface Sci. 2001;239(2):584-6.

289. Rajesh S, Nisa VS, Murali KP, Ratheesh R. Microwave dielectric properties of PTFE/rutile nanocomposites. J 
Alloys Compd. 2009;477(1-2):677-82.

290. Krishnappa M, Souza VS, Ganganagappa N, Scholten JD, Teixeira SR, Dupont J, et al. Mesoporous Foam $\mathrm{TiO}_{2}$ Nanomaterials for Effective Hydrogen Production. Chem - A Eur J. 2015;21(49):17624-30. 
Appendix A. Additional Characterization Data for the Ag-decorated $\mathrm{TiO}_{2}$ Composites: EDS, XPS.
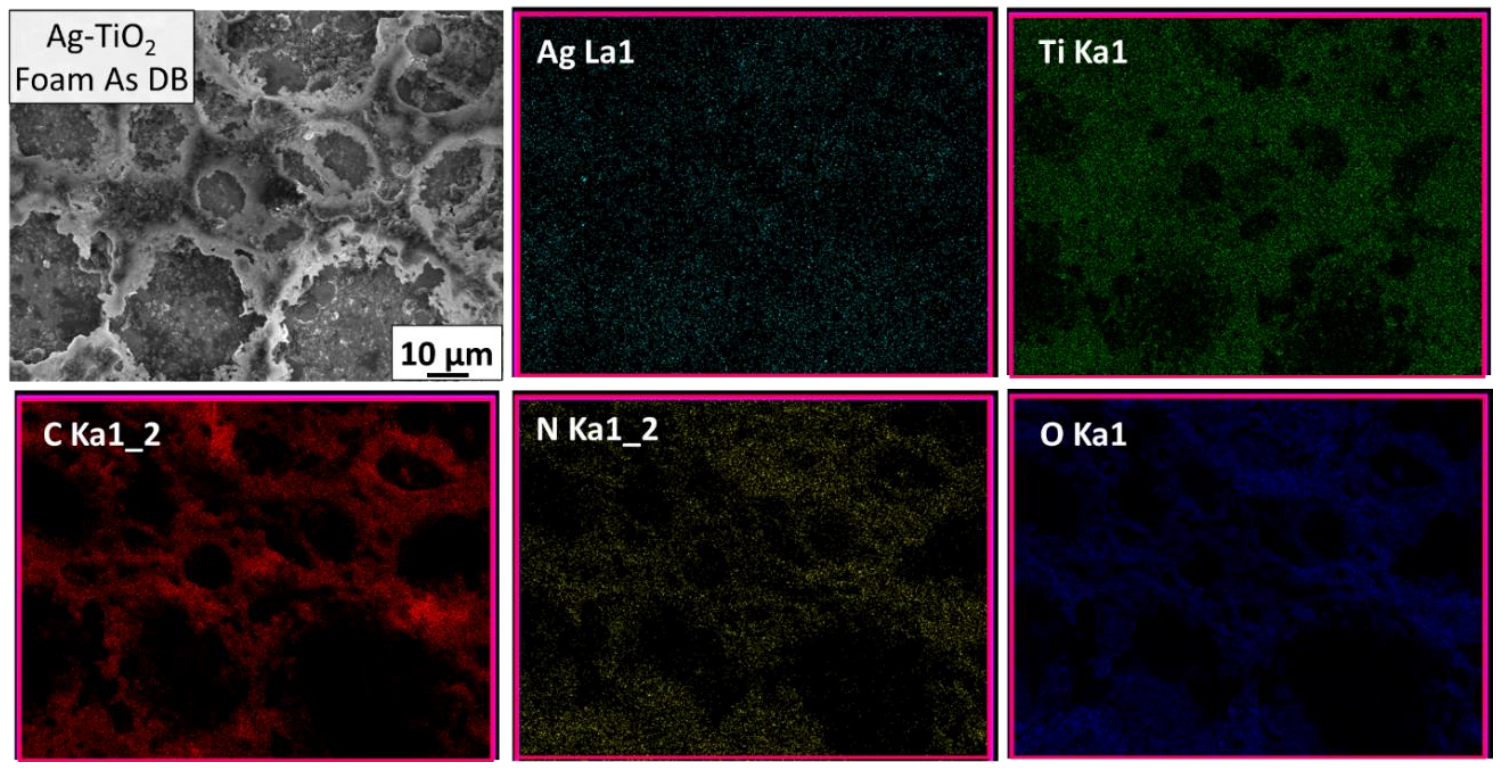

Figure A 1. EDS map of the "as doctor bladed" oil-based Ag-TiO 2 foam.

Table A 1. EDS quantitative information for the "as doctor bladed" oil-based Ag-TiO 2 foam.

\begin{tabular}{ccccccccccc}
\hline Element & (atomic \%) & $\mathbf{C}$ & $\mathbf{N}$ & $\mathbf{O}$ & $\mathbf{N a}$ & $\mathbf{S i}$ & $\mathrm{Ca}$ & $\mathrm{Ti}$ & $\mathbf{A g}$ & $\mathrm{In}$ \\
\hline \multirow{2}{*}{ EDS } & Selected Area & 59.45 & --- & 26.58 & 0.22 & 2.2 & 0.36 & 8.06 & 0 & 3.14 \\
\hline
\end{tabular}
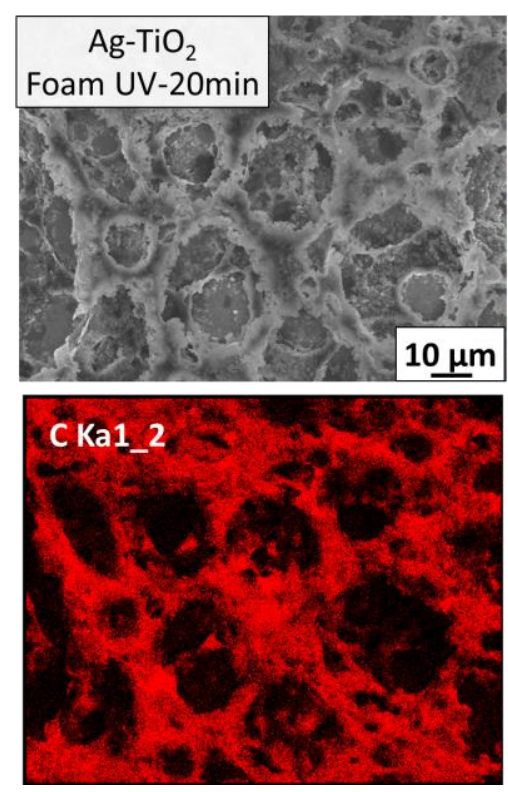

Figure A 2. EDS map of the oil-based $\mathrm{Ag}^{-T i O}{ }_{2}$ foam treated under UV-light $(\lambda=254 \mathrm{~nm})$ for $20 \mathrm{~min}$.

Table A 2. EDS quantitative information for the oil-based Ag-TiO ${ }_{2}$ foam treated under UV-light ( $\left.\lambda=254 \mathrm{~nm}\right)$ for $20 \mathrm{~min}$.

\begin{tabular}{cccccccccc}
\hline Element & (atomic \%) & $\mathbf{C}$ & $\mathbf{O}$ & $\mathrm{Na}$ & $\mathrm{Si}$ & $\mathrm{Ca}$ & $\mathrm{Ti}$ & In \\
\hline \multirow{2}{*}{ EDS } & Selected Area & 62.04 & 25.32 & 0 & 1.05 & 0.22 & 9.54 & 1.84 \\
\hline
\end{tabular}



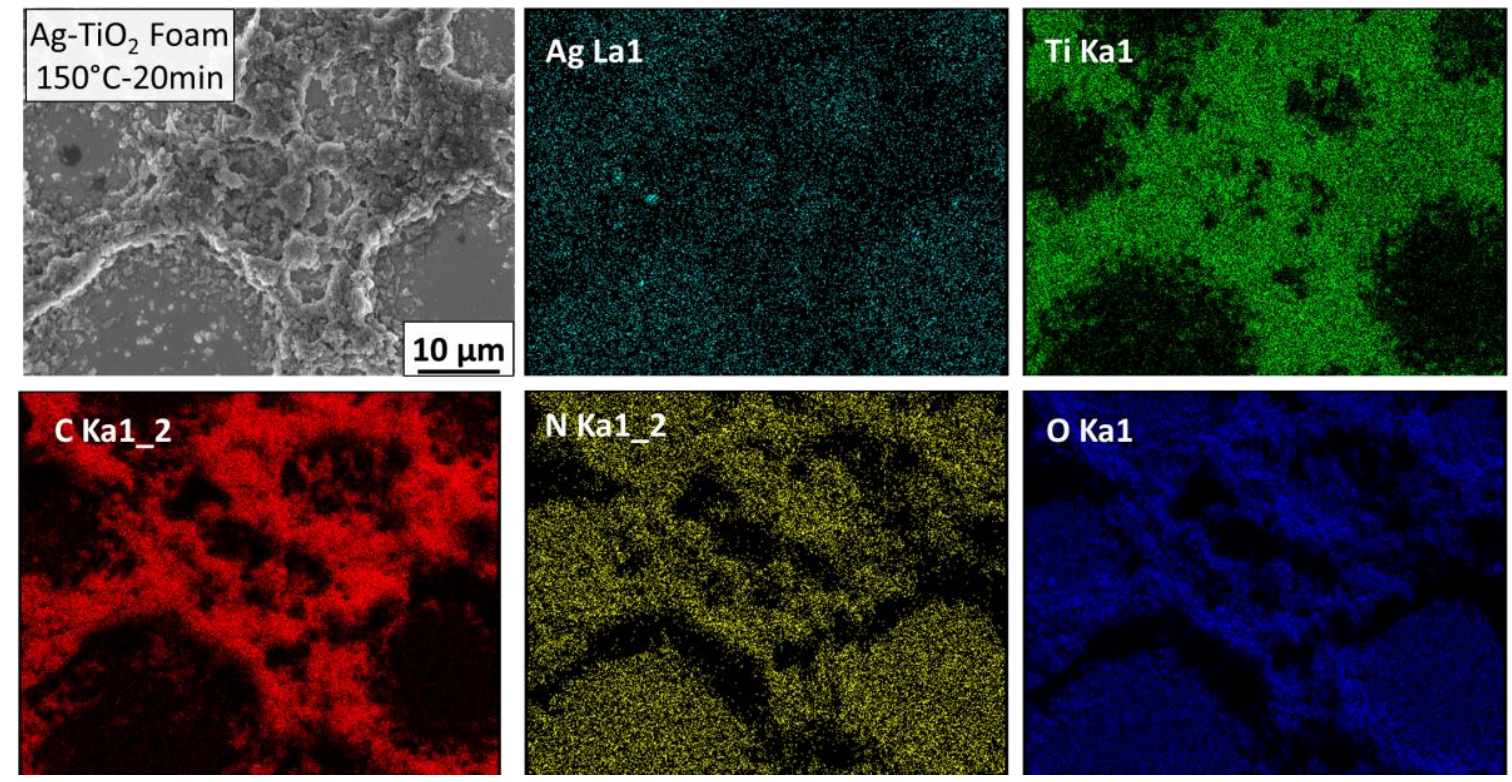

Figure A 3. EDS map of the oil-based $\mathrm{Ag}-\mathrm{TiO}_{2}$ foam treated at $150{ }^{\circ} \mathrm{C}$ for $20 \mathrm{~min}$.

Table A 3. EDS quantitative information for the oil-based $\mathrm{Ag}-\mathrm{TiO}_{2}$ foam treated at $150{ }^{\circ} \mathrm{C}$ for $20 \mathrm{~min}$.

\begin{tabular}{ccccccccc}
\hline Element & (atomic \%) & $\mathbf{C}$ & $\mathbf{O}$ & $\mathbf{N a}$ & $\mathbf{S i}$ & $\mathbf{C a}$ & $\mathrm{Ti}$ & $\mathrm{In}$ \\
\hline \multirow{2}{*}{ EDS } & Selected Area & 50.97 & 31.42 & 0.58 & 4.21 & 0.58 & 7.47 & 4.56 \\
\hline
\end{tabular}



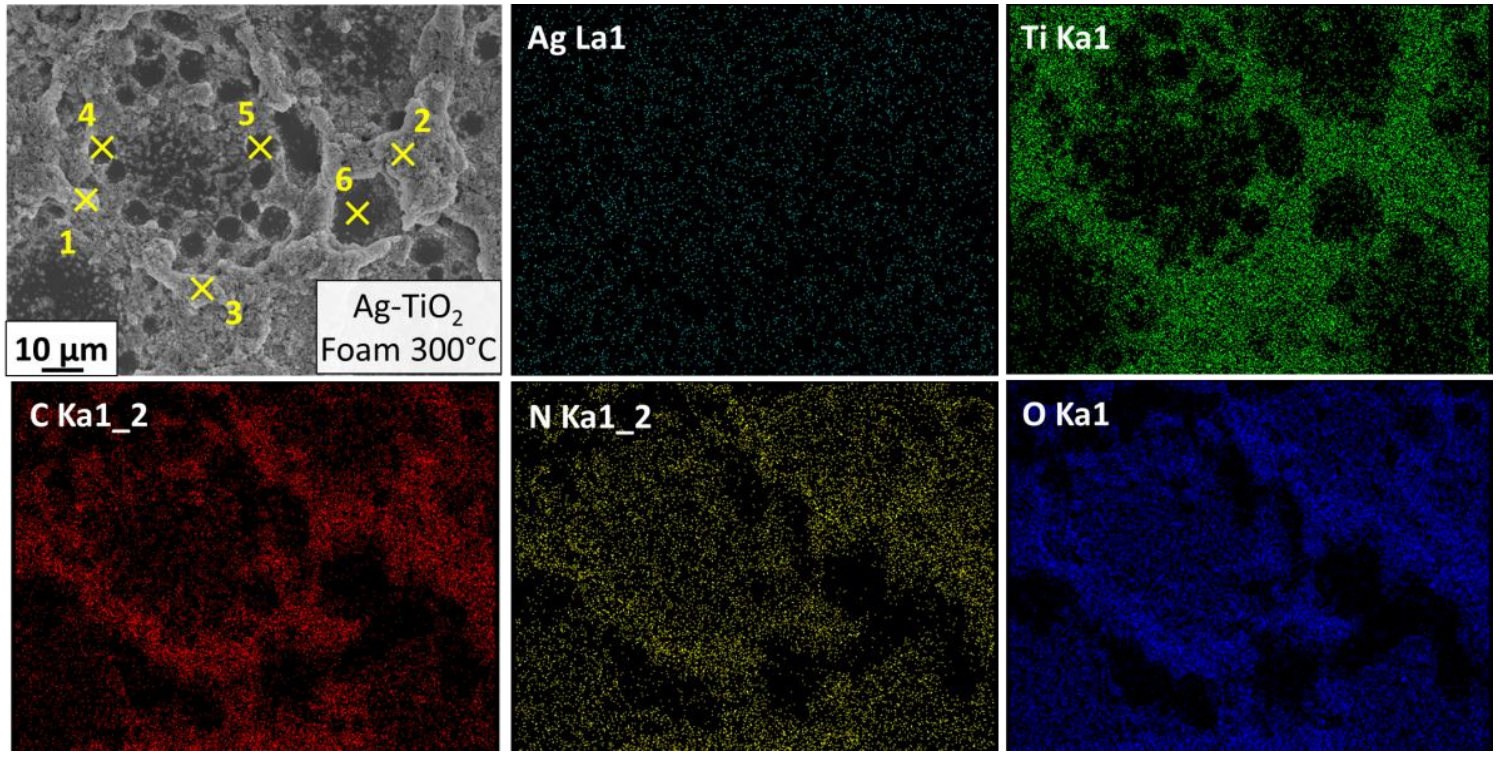

O Ka1

Figure A 4. EDS map of the oil-based $\mathrm{Ag}-\mathrm{TiO}_{2}$ foam treated at $300{ }^{\circ} \mathrm{C}$ for $20 \mathrm{~min}$.

Table A 4. EDS quantitative information for the oil-based $\mathrm{Ag}-\mathrm{TiO}_{2}$ foam treated at $300{ }^{\circ} \mathrm{C}$ for $20 \mathrm{~min}$.

\begin{tabular}{cccccccc}
\hline $\begin{array}{c}\text { Element } \\
\text { (atomic \%) }\end{array}$ & $\mathbf{1}$ & $\mathbf{2}$ & $\mathbf{3}$ & $\mathbf{4}$ & $\mathbf{5}$ & $\mathbf{6}$ & $\begin{array}{c}\text { Selected } \\
\text { Area }\end{array}$ \\
\hline $\mathbf{C}$ & 28.6 & 28.45 & 29.31 & 4.31 & 9.72 & 15.77 & 22.80 \\
$\mathbf{N}$ & 0.00 & 0.00 & 0.00 & 0.00 & 0.00 & 0.00 & 0.00 \\
$\mathbf{O}$ & 55.56 & 55.18 & 55 & 58.83 & 19.29 & 25.38 & 51.73 \\
$\mathbf{N a}$ & ---- & --- & --- & 2.27 & 1.69 & --- & 0.86 \\
$\mathbf{M g}$ & --- & --- & --- & 0.87 & 1.07 & --- & 0.29 \\
$\mathbf{S i}$ & --- & --- & --- & 17.97 & 32.98 & 22.57 & 5.48 \\
$\mathbf{C a}$ & --- & --- & --- & 1.87 & 5 & 3.99 & 0.77 \\
$\mathbf{T i}$ & 15.69 & 16.37 & 15.69 & 0.71 & 0 & 3.74 & 12.10 \\
$\mathbf{A g}$ & 0.15 & 0 & 0 & 0 & 0 & 0 & 0.13 \\
$\mathbf{I n}$ & ---- & --- & --- & 13.17 & 30.26 & 28.54 & 5.85 \\
\hline
\end{tabular}




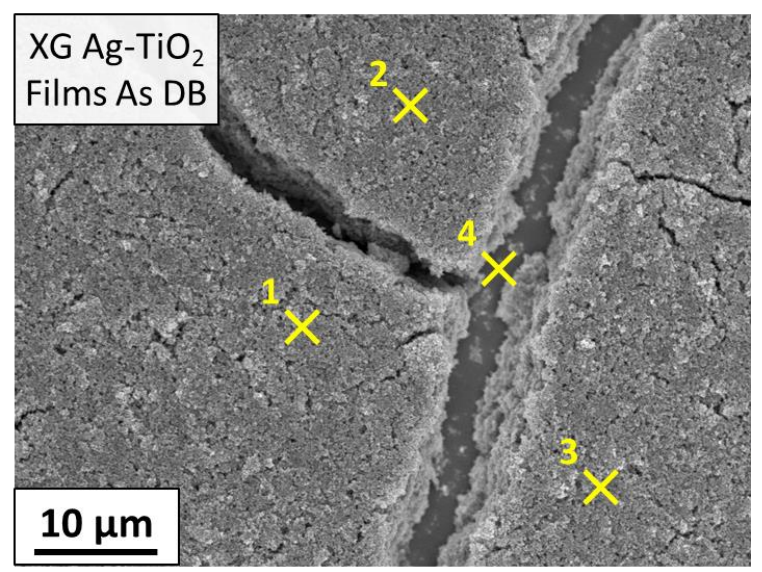

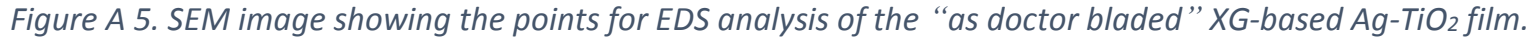

Table A 5. EDS quantitative information for the "as doctor bladed" XG-based Ag-TiO 2 film.

\begin{tabular}{ccccc}
\hline $\begin{array}{c}\text { Element } \\
\text { (atomic \%) }\end{array}$ & $\mathbf{1}$ & $\mathbf{2}$ & $\mathbf{3}$ & $\mathbf{4}$ \\
\hline $\mathbf{C}$ & 5.52 & 6.19 & 5.43 & 0 \\
$\mathbf{N}$ & 0 & --- & -- & 9.42 \\
$\mathbf{O}$ & 67.61 & 63.09 & 61.55 & 80.33 \\
$\mathbf{T i}$ & 26.6 & 33.44 & 32.71 & 10.26 \\
$\mathbf{A g}$ & 0.27 & 0.29 & 0.31 & 0 \\
\hline
\end{tabular}

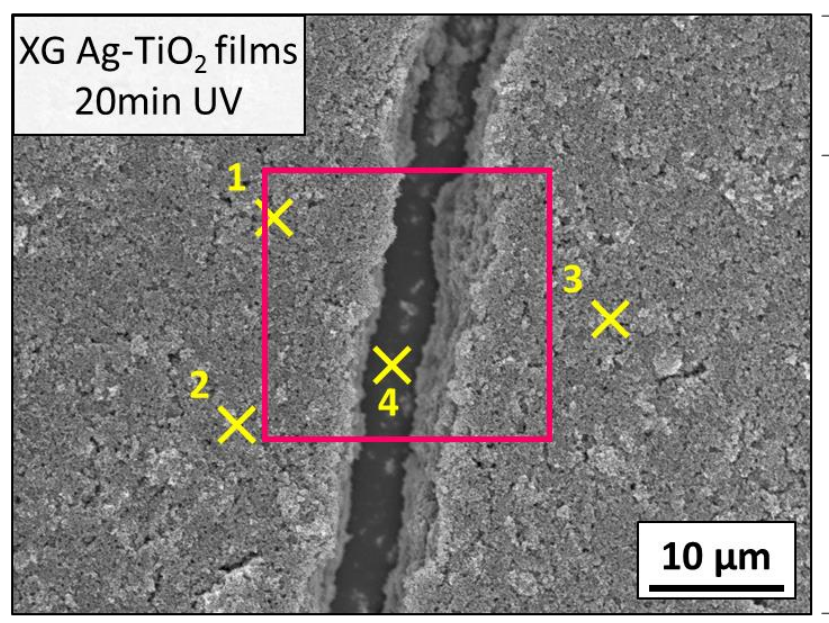

\begin{tabular}{cccccc}
\hline $\begin{array}{c}\text { Element } \\
\text { (atomic } \\
\text { \%) }\end{array}$ & $\mathbf{1}$ & $\mathbf{2}$ & $\mathbf{3}$ & $\mathbf{4}$ & $\begin{array}{c}\text { EDS } \\
\text { Selected } \\
\text { Area }\end{array}$ \\
\hline $\mathrm{C}$ & 4.73 & 4.62 & 5.69 & --- & --- \\
$\mathrm{N}$ & 0.00 & 0.00 & 0.00 & 0.00 & 0.00 \\
$\mathrm{O}$ & 54.14 & 67.32 & 57.35 & 47.21 & 66.41 \\
$\mathrm{Si}$ & --- & --- & --- & 0.47 & 1.55 \\
$\mathrm{~K}$ & --- & --- & --- & --- & 2.16 \\
$\mathrm{Ca}$ & --- & --- & --- & 3.66 & --- \\
$\mathrm{Ti}$ & 40.58 & 27.63 & 36.33 & 23.89 & 29.88 \\
$\mathrm{Ag}$ & 0.55 & 0.44 & 0.64 & --- & --- \\
$\mathrm{In}$ & --- & --- & --- & 24.77 & --- \\
\hline
\end{tabular}
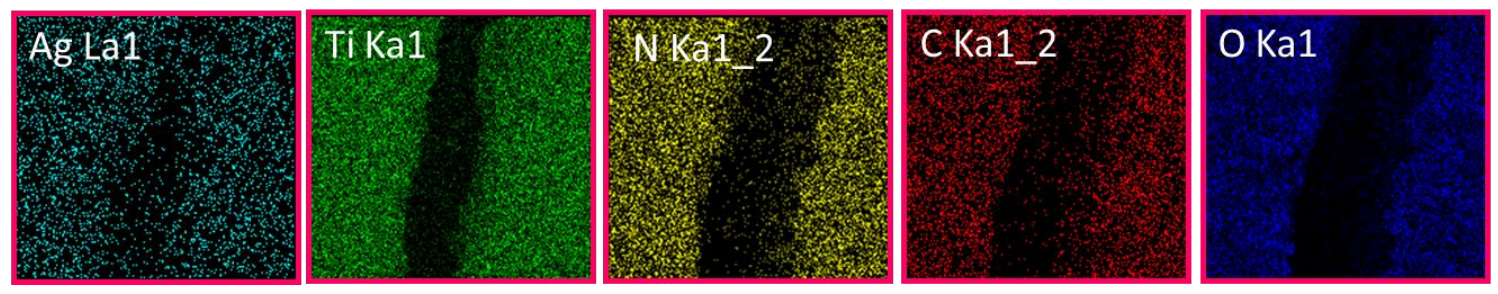

Figure A 6. EDS point analysis quantitative information and elemental map of the $\mathrm{XG}$-based $\mathrm{Ag}-\mathrm{TiO}_{2}$ film treated under UV-light $(\lambda=254 \mathrm{~nm})$ for $20 \mathrm{~min}$. 

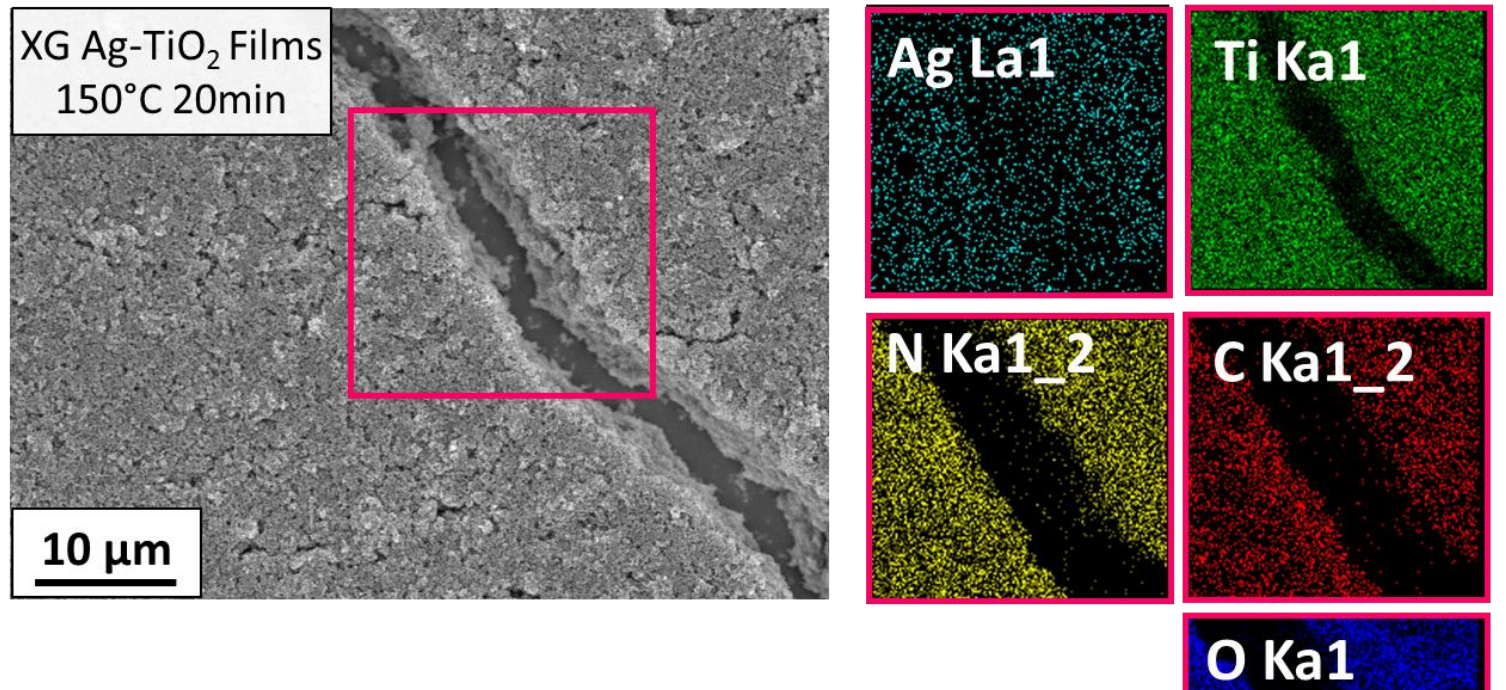

\begin{tabular}{cccccc}
\hline Element & (atomic \%) & C & O & Ti & Ag \\
\hline EDS & Selected Area & 9 & 59.97 & 31.03 & -- \\
\hline
\end{tabular}

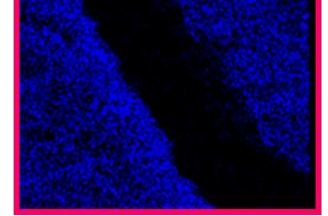

Figure A 7. EDS quantitative information and elemental map of the XG-based $\mathrm{Ag}-\mathrm{TiO}_{2}$ film treated at $150{ }^{\circ} \mathrm{C}$ for 20 $\min$.
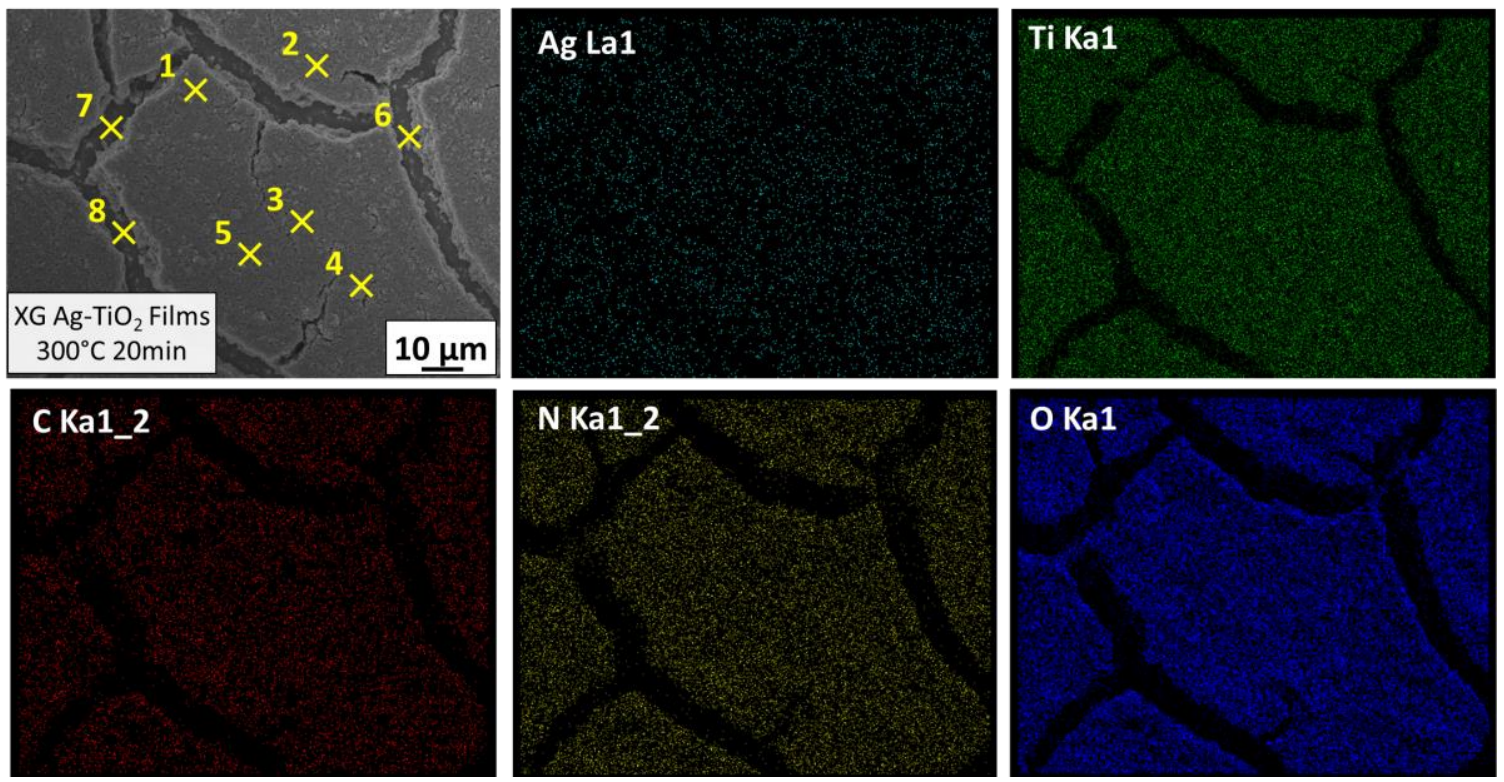

Figure A 8. EDS elemental map of the XG-based $\mathrm{Ag}-\mathrm{TiO}_{2}$ film treated at $300{ }^{\circ} \mathrm{C}$ for $20 \mathrm{~min}$. 
Table A 6. EDS quantitative information for the XG-based $\mathrm{Ag}-\mathrm{TiO}{ }_{2}$ film treated at $300{ }^{\circ} \mathrm{C}$ for 20 min.

\begin{tabular}{cccccccccc}
\hline $\begin{array}{c}\text { Element } \\
\text { (atomic } \\
\text { \%) }\end{array}$ & $\mathbf{1}$ & $\mathbf{2}$ & $\mathbf{3}$ & $\mathbf{4}$ & $\mathbf{5}$ & $\mathbf{6}$ & $\mathbf{7}$ & $\mathbf{8}$ & $\begin{array}{c}\text { Selected } \\
\text { Area }\end{array}$ \\
\hline $\mathbf{C}$ & 4.77 & 5.19 & 5.58 & 6.34 & 6.03 & 5.7 & 4.34 & 3.9 & 7.96 \\
$\mathbf{N}$ & 0 & 0 & 0 & 0 & 0 & 0 & 0 & 0 & --- \\
$\mathbf{0}$ & 62.82 & 71.19 & 68.72 & 72.54 & 67.69 & 65.44 & 35.26 & 39.12 & 61.36 \\
$\mathbf{S i}$ & --- & --- & --- & --- & --- & --- & 9.93 & 1.05 & --- \\
$\mathbf{C a}$ & --- & --- & --- & --- & --- & --- & 5.07 & 6.35 & --- \\
$\mathbf{T i}$ & 32.17 & 23.31 & 25.51 & 21.12 & 26.03 & 28.52 & 14.45 & 18.28 & 30.68 \\
$\mathbf{A g}$ & 0.24 & 0.31 & 0.19 & 0 & 0.25 & 0.34 & 0 & 0 & --- \\
In & --- & --- & --- & --- & --- & --- & 30.95 & 31.29 & --- \\
\hline
\end{tabular}



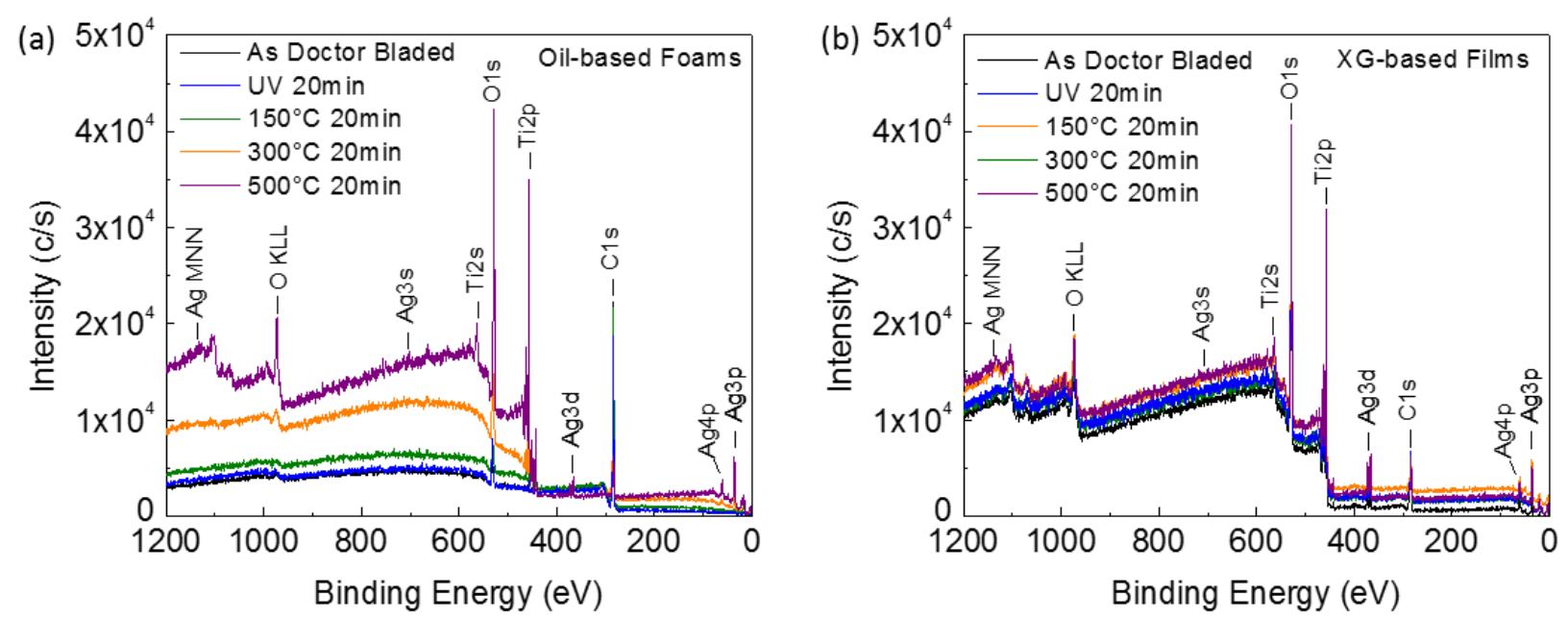

Figure A 9.X-ray photoelectron survey spectra of the $\mathrm{Ag}^{-\mathrm{TiO}_{2}}$ composites from (a) oil-based, and (b) xanthan gumbased formulations. 

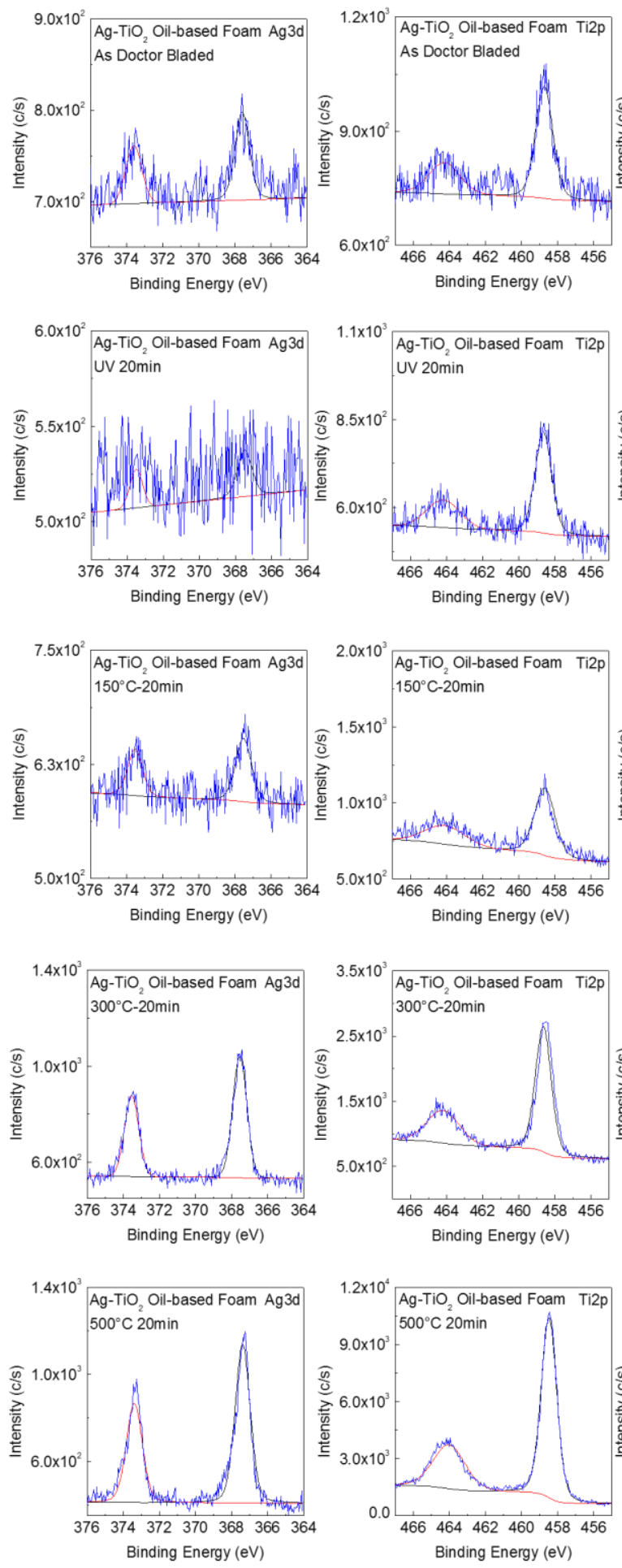
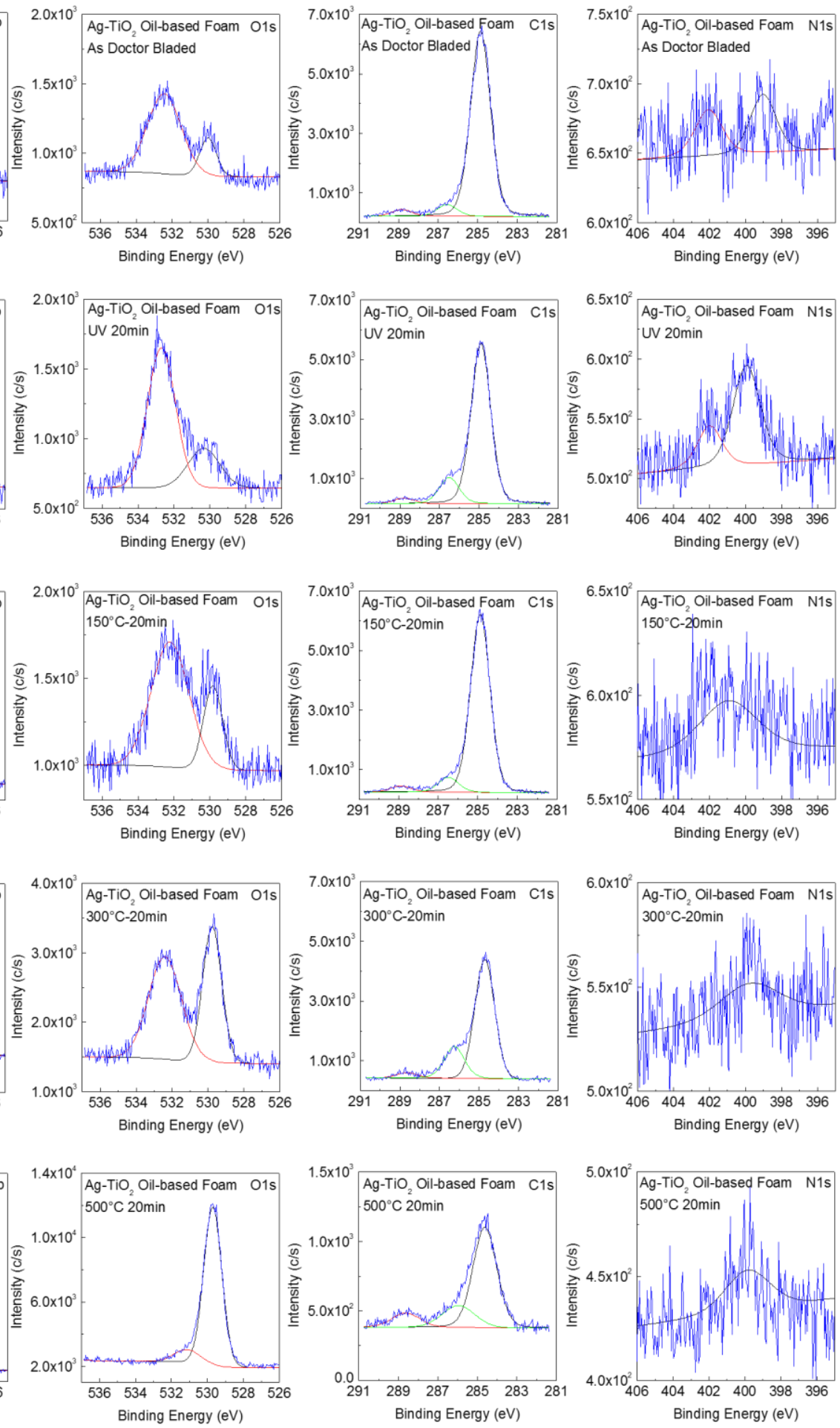

Figure A 10. XPS detailed peaks for the Ag-decorated $\mathrm{TiO}_{2}$ oil-based foams treated under different energy conditions. 

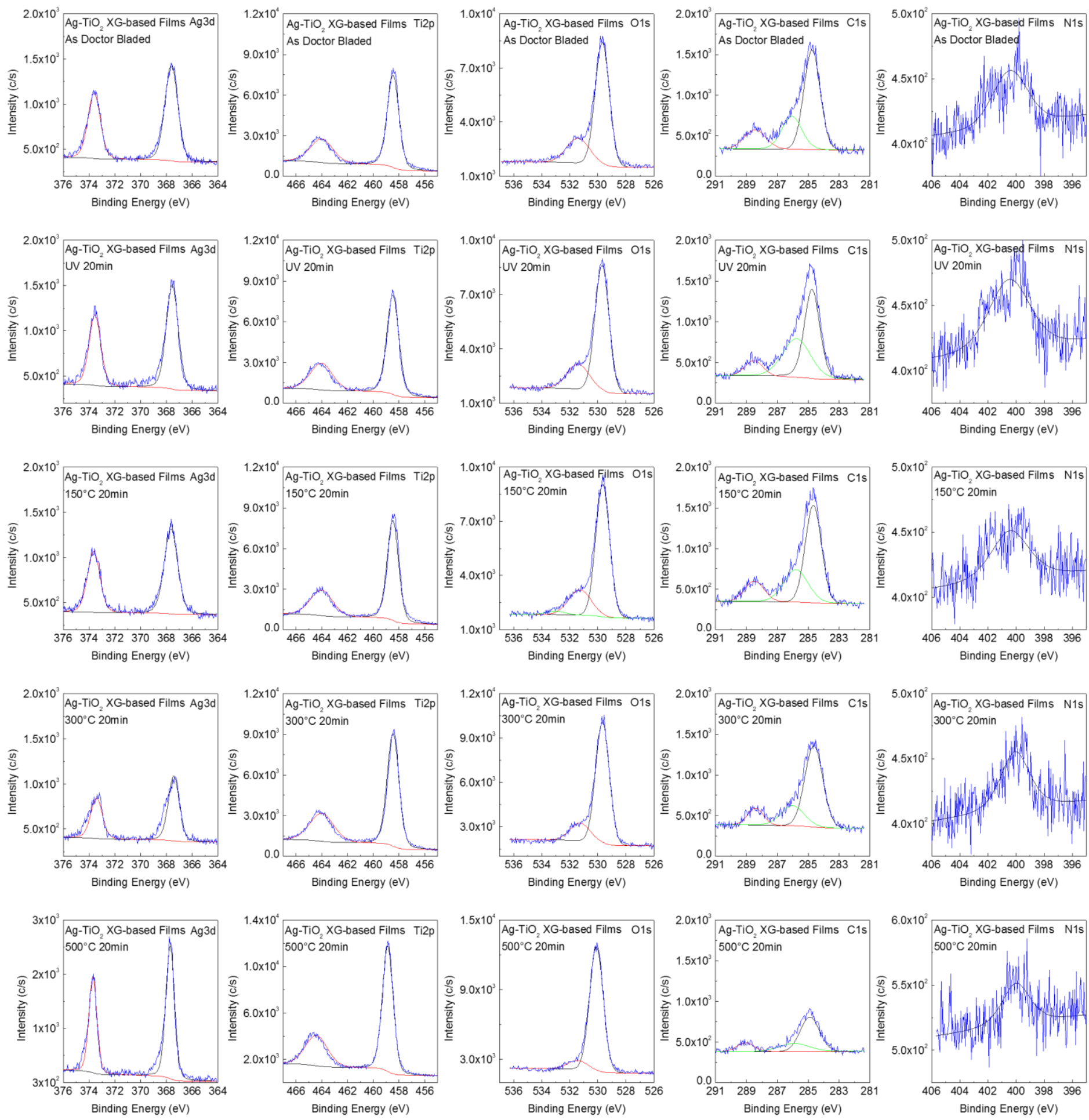

Figure A 11. XPS detailed peaks for the Ag-decorated $\mathrm{TiO}_{2}$ xanthan gum-based films treated under different energy conditions. 

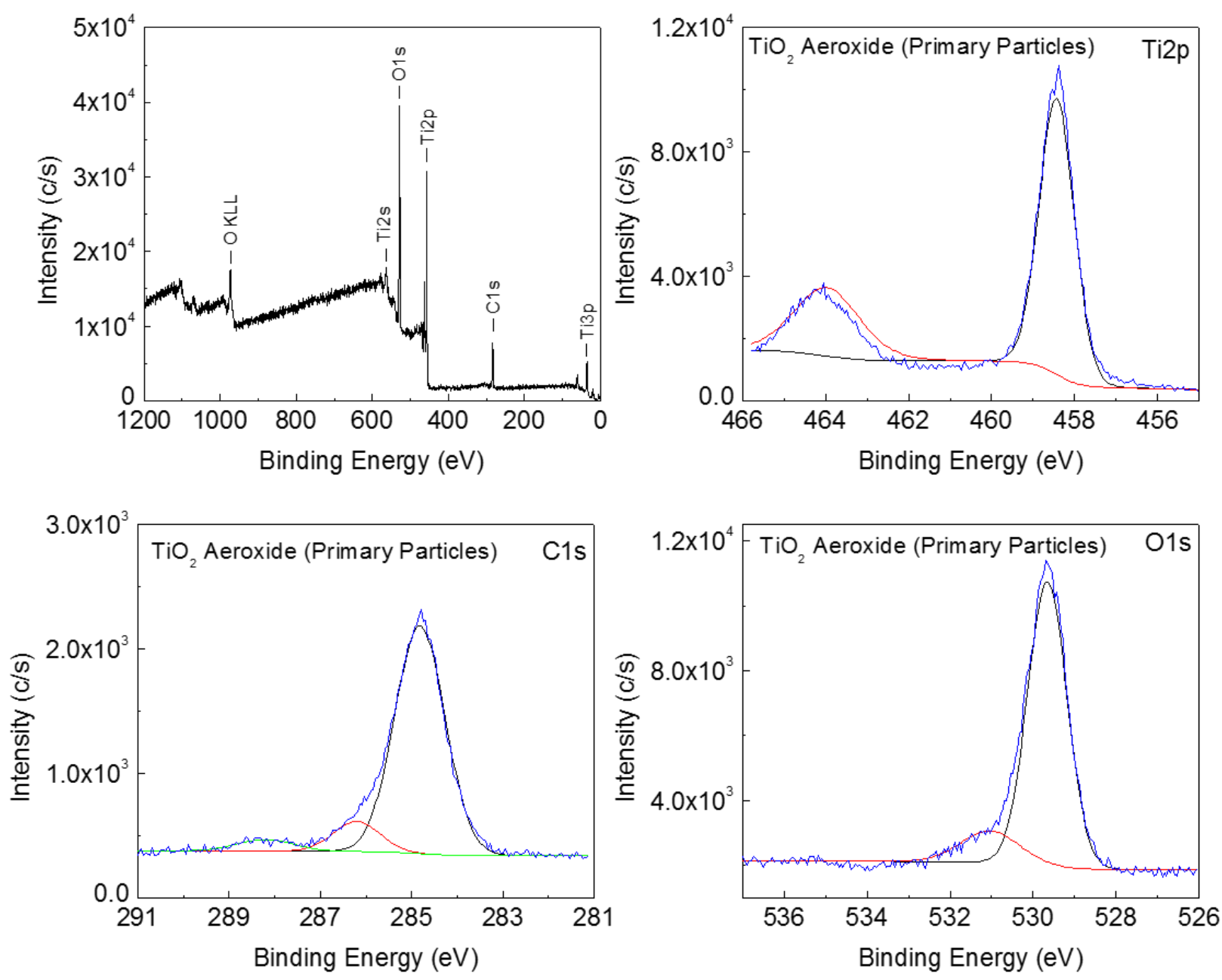

Figure A 12. X-ray photoelectron spectra for the TiO2 Aeroxide ${ }^{\circledR}$ primary particles, survey and detailed scans. 


\section{Appendix B. TiO 2 -TIAA Studies}

Important findings about the crystallization of $\mathrm{TiO}_{2}$ though hydrolysis from sol-gel reactions at room temperature from $\mathrm{Ti}$-alkoxides, suggest that the $\mathrm{Cl}$ cations play a pivotal role for the transformation of amorphous $\mathrm{TiO}_{2}$ into anatase phase $\mathrm{TiO}_{2}$ [288]. Other works [289] suggest that anatase phase is also possible if the alkoxide to water volume ratio is 1:2. Recently, the synthesis of $\mathrm{TiO}_{2}$ foams from $\mathrm{TiCl}_{4}$ in the presence of an ionic liquid was demonstrated where crystalline $\mathrm{TiO}_{2}$ is obtained from solution reactions without the need of calcination processes [290].

$\mathrm{TiO}_{2}$ emulsions from non-aqueous Titanium diisopropoxide bis(acetyl acetonate) and nanocrystalline $\mathrm{TiO}_{2}$ (Aeroxide ${ }^{\circledR} \quad \mathrm{P} 25$ ) were attempted, and a series of formulations of $\mathrm{TiO}_{2}$ :TIAA (1:0), (1:1) and (1:3) in the oil phase, and of dimethyl-sulfoxide as the apolar phase were prepared. For all of these systems, rapid crystallization occurred (within 24 hours), possibly from residual water physisorbed onto the added crystalline $\mathrm{TiO}_{2}$ particles, difficulting the printing process due to clogging of the dispensing nozzles. Filtration of the inks using a $5 \mu \mathrm{m}$ mesh filter was also attempted with unsuccessful results, the added $\mathrm{TiO}_{2}$ particles were greatly stopped by the filter reducing the particle load in the system, followed by severe
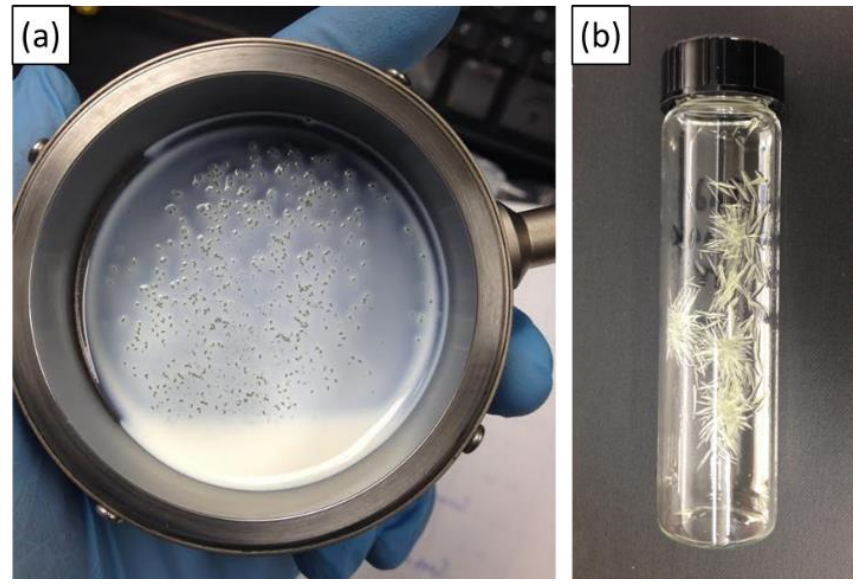

Figure B 1. Pictures of the crystal-like formations from titanium diisopropoxide bis(acetyl acetonate) (TIAA) when exposed to ambient water (a) formed during the viscosity measurement of the ink, and (b) crystals formed after 5 days from a TIAA solution in decane, DMSO and Pluronic 123. clogging of the filters half-way in the filtration process due to the large $\mathrm{TiO}_{2}$ crystals forming from the TIAA hydrolyzation and the accumulation of the added $\mathrm{TiO}_{2}$

$\mathrm{TiO}_{2}-\mathrm{TIAA}$ Ink

The titanium(IV) diisopropoxide bis(acetylacetonate) (TIAA), is a Ti-organic precursor that hydrolyses rapidly in the presence of water to form amorphous $\mathrm{TiO}_{2}$. A TIAA-based sol-gel ink to produce $\mathrm{TiO}_{2}$ at high temperatures (starting at $\sim 450^{\circ} \mathrm{C}$ )[124], is used as Ti-organic phase for the hybrid ink formulation, combined with crystalline $\mathrm{TiO}_{2}$ (Aeroxide ${ }^{\circledR}$, Sigma-Aldrich) nanoparticles, see precursors Table B 1 . 
Table B 1. TIAA Inks precursor table.

\begin{tabular}{|c|c|c|c|}
\hline Precursor & Chemical Formula & Chemical Structure & $\begin{array}{c}\text { Molecular } \\
\text { Weight }(\mathrm{g} / \mathrm{mol})\end{array}$ \\
\hline DI Water & $\mathrm{H}_{2} \mathrm{O}$ & & 18.015 \\
\hline $\begin{array}{l}\text { Titanium (IV) Diisopropoxide } \\
\text { Bis(acetylacetonate) }\end{array}$ & $\mathrm{C}_{16} \mathrm{H}_{28} \mathrm{O}_{6} \mathrm{Ti}$ & & 364.101 \\
\hline $\begin{array}{l}\text { Titanium Dioxide Nanoparticles } \\
\text { (20 nm diameter) - Aeroxide } \AA\end{array}$ & $\mathrm{TiO}_{2}$ & & 79.865 \\
\hline Polyvinylpyrrolidone & $\left(\mathrm{C}_{6} \mathrm{H}_{9} \mathrm{NO}\right)_{\mathrm{n}}$ & & 111.084 \\
\hline Polyacrylic Acid - PAA & $\left(\mathrm{C}_{3} \mathrm{H}_{4} \mathrm{O}_{2}\right)_{\mathrm{n}}$ & & 72.033 \\
\hline Ethanol & $\mathrm{CH}_{3} \mathrm{CH}_{2} \mathrm{OH}$ & & 46.07 \\
\hline Ammonium Hydroxide & $\mathrm{NH}_{4} \mathrm{OH}$ & & 35.046 \\
\hline
\end{tabular}

\section{Ink Rheology and Thermal Decomposition}

The viscosity and thermal decomposition of different TIAA based formulations was studied to explore the potential of this solution-based ink system. The viscosity of the formulated sol-gel organic inks greatly changes as polymer is added to the system as thickening agent. A Newtonian fluid behavior is observed, for both of the polymers studied. However, for the PAA added formulation the viscosity exhibits larger values. A particular deviation from such Newtonian behavior is observed as $\mathrm{TiO}_{2}$ particles are incorporated 
to the inks, where a shear-thinning behavior becomes more relevant as larger amounts of $\mathrm{TiO}_{2}$ particles are added.
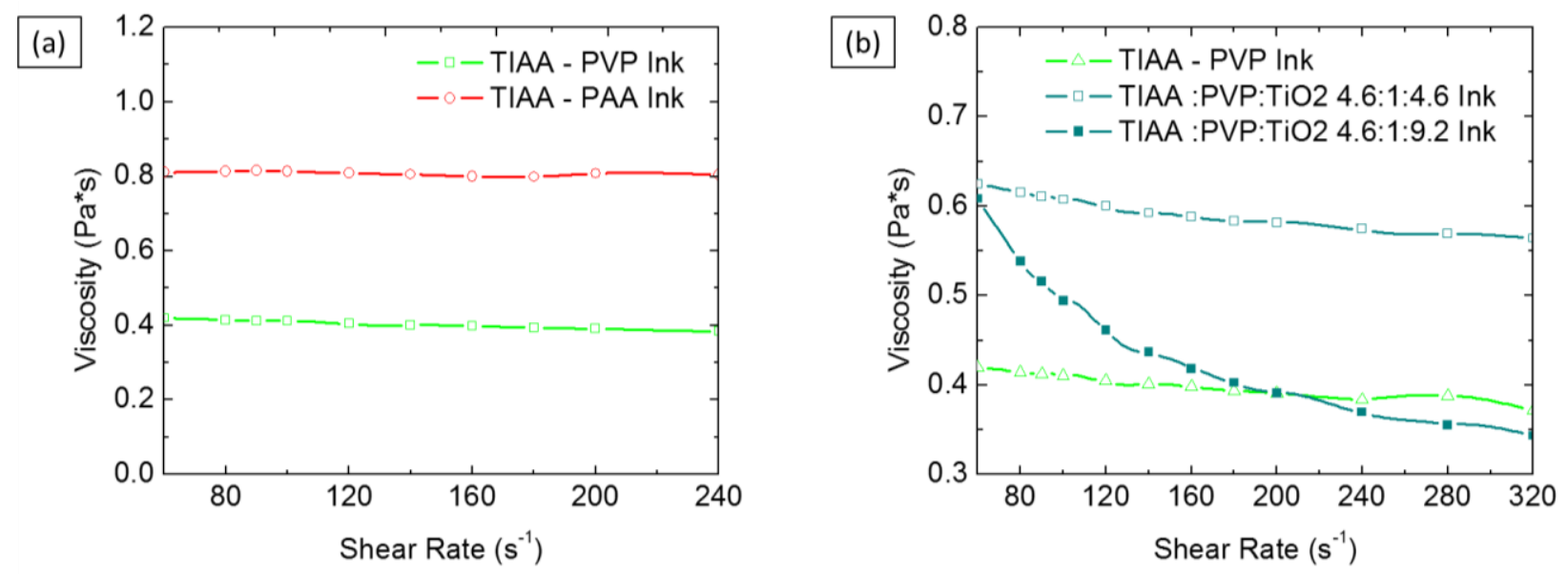

Figure B 3. Viscosity of the TIAA ink formulations (a) with different polymers and (b) for the PVP formulation with different amount of $\mathrm{TiO}_{2}$ particles.

The thermo-gravimetric analysis (TGA) of the representative TIAA-based formulations show approximate yields of $20 \% \quad \mathrm{TiO}_{2}$ upon thermal annealing, the transformation from amorphous $\mathrm{TiO}_{2}$ to anatase phase, occurs around $\sim 550^{\circ} \mathrm{C}$, as observed from the final inflection point of the TGA curves. As it is expected, for the $\mathrm{TiO}_{2}$-TIAAPVP based system, larger amounts of $\mathrm{TiO}_{2}$ solids are obtained at upon thermal annealing as more $\mathrm{TiO}_{2}$ particles are incorporated to the system. Additionally, the inflection point observed for the amorphous to anatase $\mathrm{TiO}_{2}$ transition, exhibits a shift towards lower temperatures with increasing $\mathrm{TiO}_{2}$ content, suggesting a lowering in the thermal-budget required to crystallize the ink systems as $\mathrm{TiO}_{2}$ nanoparticles are incorporated. Because the $\mathrm{TiO}_{2}$ particles' surface consists of ordered arrangements of $\mathrm{TiO}_{2}$ lattices, it is hypothesized that such ordered structure serves as nucleating sites for the crystallizing $\mathrm{TiO}_{2}$ from the TIAA precursor; thus, larger amounts of nucleation sites become available as more particles are added to the system. It is worth mentioning that, due to the hygroscopic nature of $\mathrm{TiO}_{2}$, physisorbed water at the surface of the added $\mathrm{TiO}_{2}$ particles, may in turn promote hydrolysis of the metal-oxide precursor, favoring the transition as more $\mathrm{TiO}_{2}$ particles are incorporated.
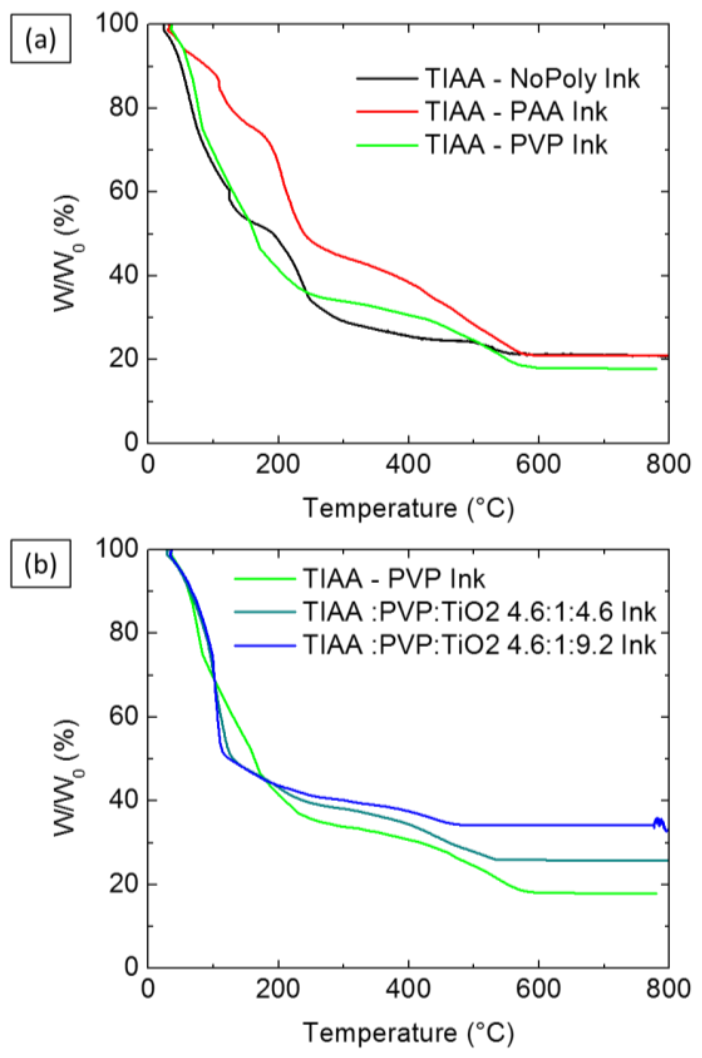

Figure B 2. TGA of the TIAA based formulations (a) with different polymers and (b) for the PVP formulations with different TiO2 particle content. 

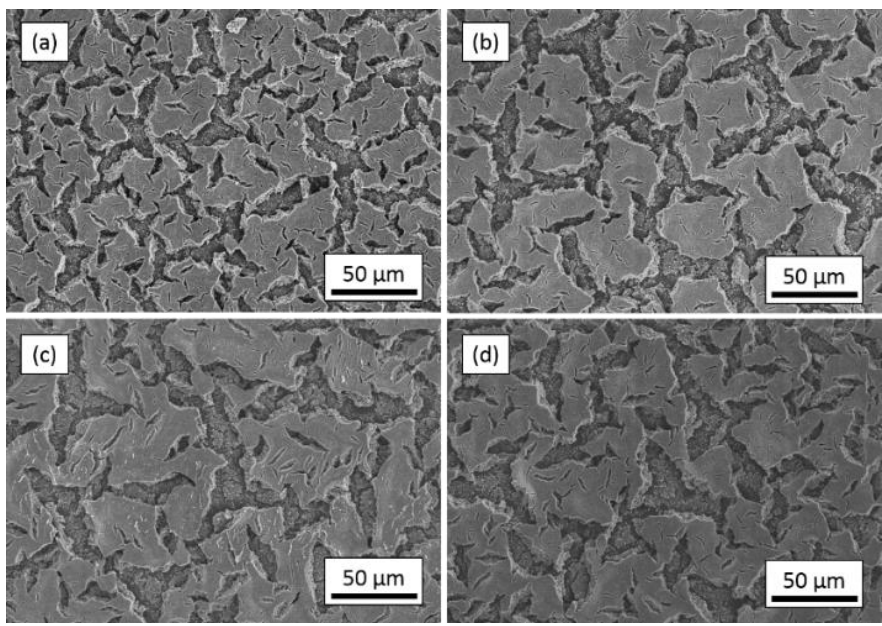

Figure B 4. SEM images of the surface of the spin coated $\mathrm{TiO}_{2}-$ Sol films after sintering at $400^{\circ} \mathrm{C}(\mathrm{a}), 450^{\circ} \mathrm{C}(\mathrm{b}), 500^{\circ} \mathrm{C}$ (c) and $550^{\circ} \mathrm{C}(d)$.

The microstructure of the films produced through spin-coating from the baseline (TIAAPVP formulation - no particles added) treated at high temperatures, are shown in Figure B 4, Figure B 5 and Figure B 6. Cracking of the films is evidenced for all the films obtained. These cracks arise from the high surface tension that develops as the ink is dried and the titanium dioxide particles crystallize. The heating rate used for the sintering processes, kept constant to $5{ }^{\circ} \mathrm{C} / \mathrm{min}$; is expected to have direct influence on the crack appearance and density, lower heating rates may result in lowering the crack density of the films. The use of Polyvinylpyrrolidone (PVP) expected to act as thickening agent and to avoid cracking[124] of the $\mathrm{TiO}_{2}$ structures during thermal annealing, is observed not to be the primary solution for avoiding cracking of such structures. Further review of the work reported by Lewis et. al.[124,125] indicates the use of a sacrificial polymeric film between the substrate and the printed $\mathrm{TiO}_{2}$ structures, which from our experience with the system in scrutiny, is what ultimately relieves the stress generated. Additionally, different shape, and dimensions - width and height of the patterns (films, straight lines, zig-zag lines, etc...) are expected to result in different surface finishes.

The structure of the spin-coated films, consists of cracked surface films with $\mathrm{TiO}_{2}$ grains underneath. Figure B 5.d shows the surface for the $450^{\circ} \mathrm{C}$ sintered film, with highly aligned whisker like layers, clearly different from the rounded grains below the surface.
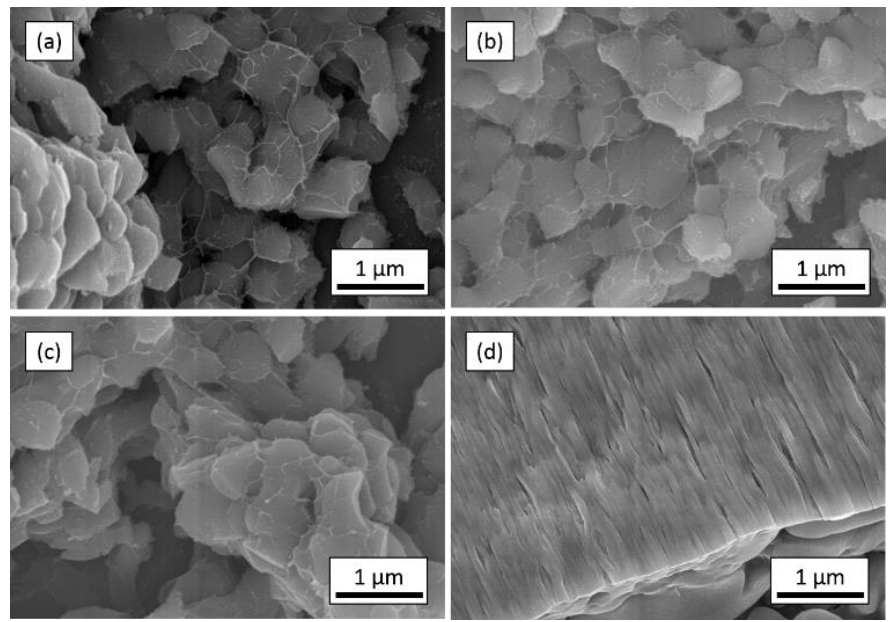

Figure $\mathrm{B} 5$. $\mathrm{TiO}_{2}$ - Sol ink, SEM images of titanium dioxide grains underneath the film surface after sintering at $400^{\circ} \mathrm{C}(\mathrm{a}), 450^{\circ} \mathrm{C}$ (b) and $500^{\circ} \mathrm{C}$ (c). Surface of the film after sintering at $450^{\circ} \mathrm{C}$ (d). 

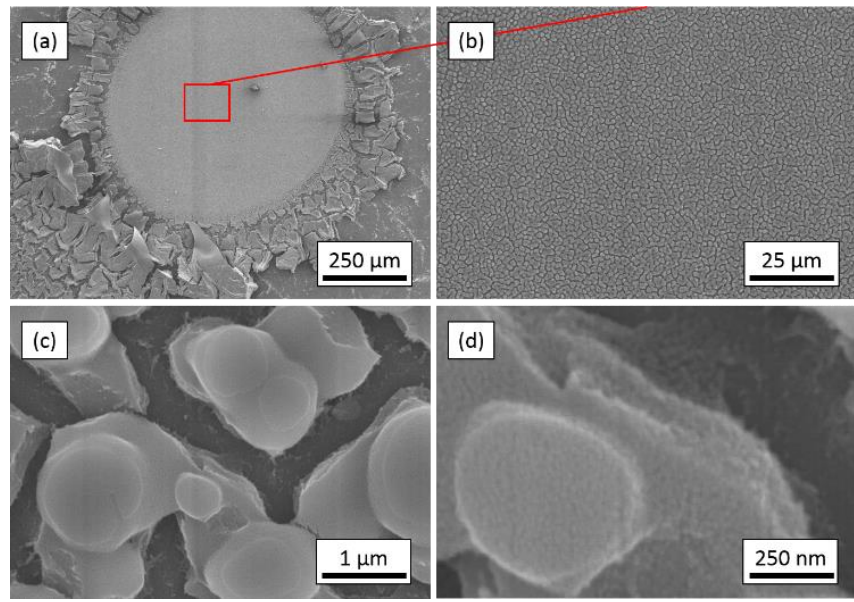

Figure B 6. SEM images of special feature in the $\mathrm{TiO}_{2}-\mathrm{Sol}$ ink spin coated film after sintering at $550^{\circ} \mathrm{C}$.

The XRD patterns for the titanium solution films sintered at different temperatures from $400^{\circ} \mathrm{C}$ to $550^{\circ} \mathrm{C}$ are shown in Figure $\mathrm{B}$ 7. Diffraction from $\mathrm{TiO}_{2}$ anatase phase is observed. As the sintering temperature is increased, narrowing of the diffraction peak for the (011) planes is appreciated, suggesting preferential orientation accommodation of the crystal planes in the titanium dioxide particles with higher temperatures during sintering. Stronger anatase peaks with increasing temperature indicates that it is the dominating phase. The peak identified as $\mathrm{R}^{*}$ can be assigned to $\mathrm{TiO}_{2}$ rutile phase and is observed to become more pronounced as the annealing temperature increases; however, the absence of the main rutile peak at $\sim 27.4^{\circ} 2 \theta$ angle, suggests that preferential orientation of such $\mathrm{TiO}_{2}$ phase may occur.

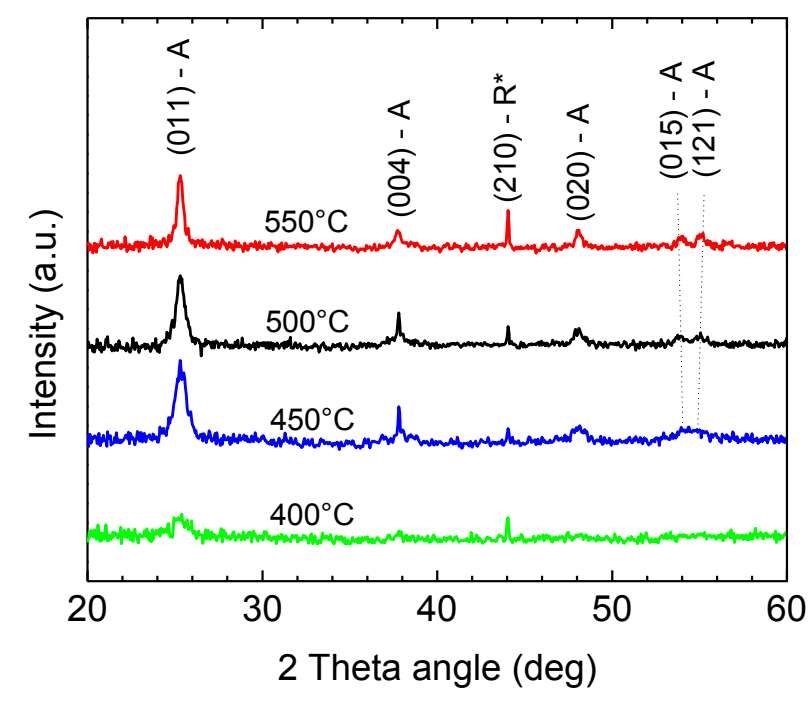

Figure B 7. XRD patterns of the spin coated $\mathrm{TiO}_{2}-\mathrm{Sol}$ films after different sintering temperatures.

\section{Challenges from the TIAA Systems}

For all the TIAA based formulations, rapid sedimentation of amorphous $\mathrm{TiO}_{2}$ crystals occurs. The different polymers affect the hydrolysis rate of the TIAA to a greater or lesser extent; nevertheless, the instability of this $\mathrm{TiO}_{2}$ precursor make the inks' rheological properties to change within minutes, difficulting their manipulation in open-air conditions. The clear TIAA inks (no particles added) would turn cloudy as soon as the vials were opened; whereas for the $\mathrm{TiO}_{2}$ particle added formulations, spontaneous solidification of the inks and clogging of the nozzles was almost immediate when robotic printing was attempted. For the PVP formulations (the most stable), spontaneous crystallization occurred approximately within 6-24 hours after the inks were prepared. Alternatively, the synthesis of the TIAA inks in a water-free environment may solve for this issue allowing the implementation of these systems. 


\section{Concluding Remarks - $\mathrm{TiO}_{2}-\mathrm{TIAA}$ System}

The crystallization of $\mathrm{TiO}_{2}$ films from sol-gel formulations of TIAA was investigated by varying the annealing temperature administered to spin-coated films. The treatments lead to different crystallinity grades as observed from the diffraction studies. The incorporation of $\mathrm{TiO}_{2}$ nanoparticles to the sol-gel TIAA formulation causes rapid crystal formations, attributed to forming $\mathrm{TiO}_{2}$ structures from hydrolysis of the TIAA.

The synthesis, rheological and thermal decomposition studies of the different $\mathrm{TiO}_{2}$-TIAA formulations, confirm the importance of the different polymers used as well as the $\mathrm{TiO}_{2}$ nanoparticle concentration. The large increase in surface area, provided by the $\mathrm{TiO}_{2}$ nanoparticles, is considered to be the main reason for having such an accelerated hydrolysis of the TIAA precursor in the inks. Synthesis of these in controlled atmospheres, in the absence of water molecules, is identified as a potential solution for the realization of stable hybrid ink formulations. 